\title{
Studies in Perception and Action X
}

FIFTEENTH INTERNATIONAL CONFERENCE ON PERCEPTION AND ACTION

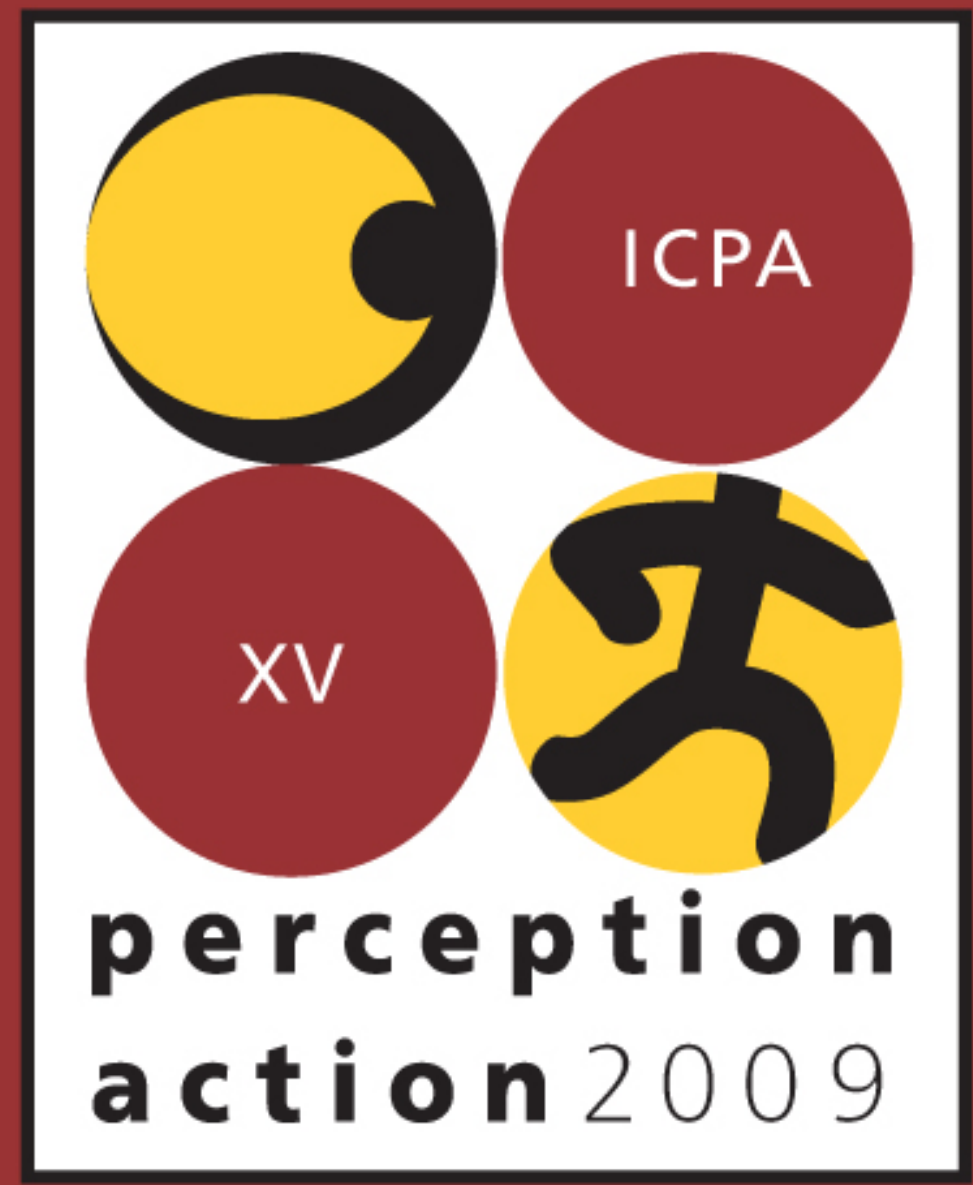

EDITED BY

Jeffrey B. Wagman and Christopher C. Pagano 


\title{
Studies in Perception and Action X
}

\section{FIFTEENTH INTERNATIONAL CONFERENCE ON PERCEPTION AND ACTION}

\author{
July 12-17, 2009 \\ Minneapolis, Minnesota
}

EDITED BY

Jeffrey B. Wagman and Christopher C. Pagano 
Cover design by Rally Pagulayan

Psychology Press

Taylor \& Francis Group

270 Madison Avenue

New York, NY 10016
Psychology Press

Taylor \& Francis Group

27 Church Road

Hove, East Sussex BN3 2FA

(C) 2009 by Taylor and Francis Group, LLC

Psychology Press is an imprint of Taylor \& Francis Group, an Informa business

Printed in the United States of America on acid-free paper

10987654321

International Standard Book Number: 978-1-84872-880-6 (Paperback)

For permission to photocopy or use material electronically from this work, please access www. copyright.com (http://www.copyright.com/) or contact the Copyright Clearance Center, Inc. (CCC), 222 Rosewood Drive, Danvers, MA 01923, 978-750-8400. CCC is a not-for-profit organization that provides licenses and registration for a variety of users. For organizations that have been granted a photocopy license by the CCC, a separate system of payment has been arranged.

Trademark Notice: Product or corporate names may be trademarks or registered trademarks, and are used only for identification and explanation without intent to infringe.

\section{Library of Congress Cataloging-in-Publication Data}

International Conference on Perception and Action (15th : 2009 : Minneapolis, Minn.)

Studies in perception and action $\mathrm{X}$ : fifteenth International Conference on

Perception and Action / [edited by] Jeffrey B. Wagman, Christopher C. Pagano. p. cm.

"Contains research presented at the ICPA XV conference held in Minneapolis, Minnesota from July 12-17, 2009"--Preface.

Includes bibliographical references.

ISBN 978-1-84872-880-6 (pbk. : alk. paper)

1. Perceptual-motor processes--Congresses. I. Wagman, Jeffrey B. II. Pagano, Christopher C. III. Title. IV. Title: Studies in perception and action 10.

BF295.I58 2010

153.7--dc22

Visit the Taylor \& Francis Web site at http://www.taylorandfrancis.com

and the Psychology Press Web site at http://www.psypress.com 


\section{Table of Contents}

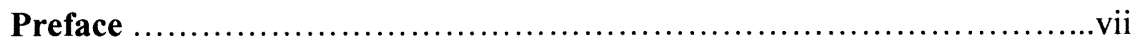

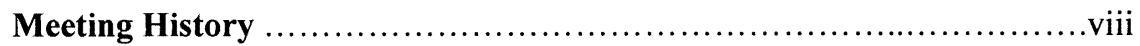

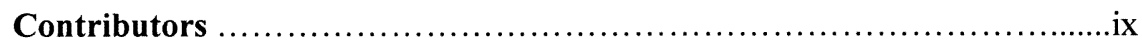

\section{Chapter 1: Action \& Coordination}

Nonlinear Attractor Dynamics and Symmetry Breaking in Prism Adaptation and Re-adaptation

Julia J. C. Blau, Damian G. Stephen, Till D. Frank, Michael T. Turvey \& Claudia Carello

Functional Tuning of Action to Task Constraints in Tool-Use: The Case of Stone Knapping

Blandine Bril, Robert Rein \& Tetsushi Nonaka

Variability of Uni-Manual Pendulum Oscillation at and away from

Resonance

Dobromir G. Dotov, Damian G. Stephen, Till D. Frank \&

Michael T. Turvey

Comparison of Performance of Tray-Carrying Task by Persons with

Down Syndrome and Those with Other Forms of Mental Retardation

Shogo Hirata, Hideyuki Okuzumi, Mitsuru Kokubun \& Masayuki Kumai .

Observation of Action Slips in a Young Child

Naoya Hirose

Relationship between Attention and Stepping

Yoshifumi Ikeda, Shogo Hirata, Hideyuki Okuzumi \& Mitsuru Kokubun.

Relationship between Stepping and Its Tempo

Yu Kamiyama, Shogo Hirata, Hideyuki Okuzumi \& Mitsuru Kokubun

Dynamics of Motor Performance in Visually Guided Force Production

Nikita A. Kuznetsov \& Michael A. Riley

Microslips in a Manual Placing Task

Ken Yoshida \& Thomas A. Stoffregen 


\section{Chapter 2: Interpersonal Coordination}

Perceiving Affordances for a Dyad

Tehran J. Davis, Michael A. Riley, Kevin Shockley, Kimberly Capehart \&

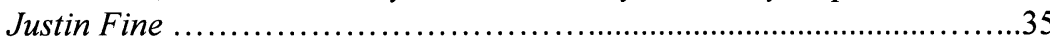

Inter- and Intra-personal Coordination in Autistic and Typically-Developing Children

Robert W. Isenhower, Kerry L. Marsh, Paula Silva, Michael J.

Richardson \& R. C. Schmidt

The Influence of Interpersonal Interaction on Postural Coordination

Dynamics in a Suprapostural Task

Manuel Varlet, Ludovic Marin, Julien Lagarde \& Benoît G. Bardy

Gaze Coordination between Car Drivers and Passengers: An Observation

Hiroshi Inou, Mamoru Sawada \& Hiroyuki Mishima

Target Distance Influences Interpersonal Postural Coordination

Alison Smith, Christie Pelzer, M. Russell Giveans, Kevin Shockley \&

Thomas A. Stoffregen

\section{Chapter 3: Perception \& Perceptual Learning}

Discrimination of Surfaces by Touching: Impact of Obstacles Attached to the Fingertip upon Performance

Nobuhiro Furuyama, Hiroyuki Mishima, Shin Maruyama \& Masashi Takiyama

Integration of Visuo-Haptic Information for Judging Object Size

Chia-Hao Lu, Joshua Aman \& Jüergen Konczak

Perceiving by Dynamic Touch with and without Hands

Zsolt Palatinus, Claudia Carello \& Michael T. Turvey

Apparent Point of Rotation Manipulation Affects Multimodal

Heaviness Perception

Laura Bachus, Kevin Shockley \& Michael A. Riley

Twelve-Month-Olds' Discrimination of Monkey Faces: Evidence for

Perceptual Narrowing?

Ross Flom, Melissa Wright, Amanda Phillippi, Tom Beckstead,

Jacob Jones, Harrison Allen \& Danny Boysen

Human Sensitivity to Local Taus

Nam-Gyoon Kim \& Keonho Shin 
Analysis of User's Action for Perceiving Shapes Using an Active

Perception Device

Ryo Mizuno, Kiyohide Ito \& Makoto Okamoto................................83

Improvements in Perceptual Accuracy Scale to Frequency of Feedback about Task Performance

Jeffrey B. Wagman \& Dawn M. McBride .88

The Influence of Eyeheight and Optic flow on Egocentric Distance Perception

Jonathan Shook, Eliah J. White \& Kevin Shockley .92

\section{Chapter 4: Perception of Affordances}

Sensitivity to Changes in Action Capabilities

Dilip N. Athreya, Michael A. Riley, Tehran J. Davis \&

Veronica C. Ramenzoni

Understanding the Dynamics of Behavioral Transitions in

Affordance Experiments

Stacy M. Lopresti-Goodman, Till D. Frank, Michael J. Richardson \& Michael T. Turvey.

Differences between American and Chinese Students in Perceiving Multiple Affordances for Common Objects

Lin Ye, Wilson Cardwell, Stephanie Karges, Milena Petrovic \& Leonard S. Mark

Changes in Anterior Deltoid Muscle Activity at the Preferred Critical

Boundary for Reaching

Milena Petrovic, Adam Strang \& Leonard S. Mark

Development, Balance and Flexibility as Constraints on Perceiving

Affordances

Peter B. Pufall \& Kathryn Hobbs

Ecometrics of Step-Over Height

Masaki Ueno, Shogo Hirata, Hideyuki Okuzumi \& Mitsuru Kokubun

Investigating the Information Used to Detect an Affordance for

Maximum Distance Throws

Qin Zhu \& Geoffrey Bingham 


\section{Chapter 5: Posture}

A Closed-Loop Controller to Model Postural Coordination

$V$. Bonnet, Benoît Bardy, P. Fraisse, N. Ramdani, Julien Lagarde \& S. Ramdani

Postural Stabilization of Visual Performance at Sea

Fu-Chen Chen, Yawen Yu, Sebastien Villard \& Thomas A. Stoffregen.

Immediate Awareness of the Surrounding Environment in the Form of

Optical Pushes

Makoto Inagami \& Ryuzo Ohno

Postural Response to a Suprapostural Task in Children at Risk for Developmental Coordination Disorder

Azizah J. Jor'dan, Michael G. Wade, Ken Yoshida \&

Thomas A. Stoffregen

Consequence of Internally Versus Externally Generated Visual Motion on

Postural Regulation in a Virtual Environment: The Importance of

Perception and Action Rather than Perception and Reaction

Eric Littman, Edward W. Otten \& L. James Smart, Jr.

The Effect of Open vs. Closed-Loop Optic Flow on Visually Induced Motion Sickness

Edward W. Otten \& L. James Smart, Jr.

Preferred Stance Width at Sea

Thomas A. Stoffregen, Yawen Yu, Fu-Chen Chen \& Sebastien Villard

Infants' Visually Induced Postural Sway: The Effect of the Attractiveness of Fixation Mark

Aki Tsuruhara, Yasunobu Katsumata, Michiteru Kitazaki, Akitoshi Hanazawa, So Kanazawa \& Masami Yamaguchi

Body Sway in the Processes of Perceiving Various Visual Environmental Structures

Chih-Mei (Melvin) Yang \& Chia-Chun Huang.

Body Sway and Visual Performance at Sea

Yawen Yu, Yasunobu Katsumata, \& Thomas A. Stoffregen.

Author index

Keyword index 


\section{Preface}

The edited book series Studies in Perception and Action contains a collection of research presented as posters at the International Conference on Perception and Action (ICPA). The Studies series has appeared in conjunction with the biennial ICPA since 1991 when the conference was held in Amsterdam. The ICPA conference series provides a forum for presenting new data, theory, and methodology relevant to the ecological approach to perception-action. The Studies series is unique in that it provides a peer-reviewed mechanism for documenting these developments through brief articles that contain more detail than typical conference proceedings abstracts (e.g., method and results sections) but less detail than full-length book chapters or journal articles. As a result, the Studies series makes the ICPA conference proceedings available to a wide audience in a complete yet compact format. In many instances, the contributions to Studies represent the first appearance of new ideas in a scientific venue. As a result, the Studies volumes contain the most recent and cutting-edge research in perception and action.

This volume is the $10^{\text {th }}$ in the Studies in Perception and Action series, and it contains research presented at the ICPA XV conference held in Minneapolis, Minnesota from July 12-17, 2009. The conference organizing committee co-chairs for ICPA XV were Tom Stoffregen and Mike Wade, both of the University of Minnesota. The 40 papers presented in this volume, reviewed by a scientific committee composed of distinguished perception-action researchers from six countries across four continents, represent the latest developments in ecological psychology research from laboratories around the world.

A significant challenge we faced in assembling this collection was organizing these diverse papers into coherent topic headings. Although many of the papers cut across topics (as is often the case with research in perceptionaction), we have done our best to organize the papers in a way that provides an initial structure for the reader. We have provided a list of keywords to assist the reader identifying papers on particular topics.

We thank William Mace of the International Society for Ecological Psychology (ISEP) for his support, and we thank Rally Pagulayan for the cover design. We are indebted to previous editors Sarah Cummins-Sebree, Michael A. Riley, and Kevin Shockley for their guidance and advice.

Jeffrey B. Wagman

Department of Psychology, Illinois State University

Christopher C. Pagano

Department of Psychology, Clemson University 


\section{Meeting History}

1. 1981 - Storrs, CT, USA

2. 1983 - Nashville, TN, USA

3. 1985 - Uppsala, SWEDEN

4. 1987 - Trieste, ITALY

5. 1989 - Miami, OH, USA

6. 1991 - Amsterdam, THE NETHERLANDS

7. 1993 - Vancouver, CANADA

8. 1995 - Marseilles, FRANCE

9. 1997 - Toronto, CANADA

10. $1999-$ Edinburgh, SCOTLAND

11. 2001 - Storrs, CT, USA

12. 2003 - Gold Coast, QLD, AUSTRALIA

13. 2005 - Monterey, CA, USA

14. 2007 - Yokohama City, JAPAN

15. 2009 - Minneapolis, MN, USA 


\section{Contributors}

Harrison Allen, Department of Psychology, Brigham Young University

Joshua Aman, Human Sensorimotor Control Lab, School of Kinesiology, University of Minnesota, aman0038@umn.edu

Dilip N. Athreya, Department of Psychology, University of Cincinnati, athreyadilip@yahoo.com

Laura Bachus, University of Cincinnati, BachusLE@email.uc.edu

Benoît G. Bardy, Motor Efficiency and Deficiency Laboratory, University Montpellier 1, benoit.bardy@univ-montp1.fr

Tom Beckstead, Department of Psychology, Brigham Young University

Geoffrey Bingham, Department of Psychological and Brain Sciences, Indiana University, gbingham@indiana.edu

Julia J. C. Blau, Department of Psychology, University of Connecticut, Julia.Carroll@uconn.edu

V. Bonnet, University Montpellier II, France, bonnet@lirmm.com

Danny Boysen, Department of Psychology, Brigham Young University

Blandine Bril, École des Hautes Études en Sciences Sociales - Groupe de recherché "Apprentissage et Contexte," Paris, France, blandine.bril@ehess.fr

Kimberly Capehart, Department of Psychology, University of Cincinnati

Wilson Cardwell, Department of Psychology, Center for Ergonomic Research, Miami University, cardwewb@muohio.edu

Claudia Carello, Center for the Ecological Study of Perception and Action, University of Connecticut, claudia.carello@uconn.edu

Fu-Chen Chen, University of Minnesota, chen1619@umn.edu

Tehran J. Davis, Department of Psychology, University of Cincinnati, davtj@email.uc.edu 
Dobromir G. Dotov, Department of Psychology, University of Connecticut, dobromir.dotov@uconn.edu

Justin Fine, Department of Psychology, University of Cincinnati

Ross Flom, Department of Psychology, Brigham Young University

P. Fraisse, University Montpellier II, France, fraisse@lirmm.fr

Till D. Frank, Center for the Ecological Study of Perception and Action, University of Connecticut, Till.Frank@uconn.edu

Nobuhiro Furuyama, National Institute of Informatics, furuyama@nii.ac.jp

M. Russell Giveans, University of Minnesota, givea017@umn.edu

Akitoshi Hanazawa, Kyushu Institute of Technology, Japan

Shogo Hirata, Tokyo Gakugei University, sghirata@hotmail.co.jp

Naoya Hirose, Kyoto Notre Dame University, nhirose@notredame.ac.jp

Kathryn Hobbs, Psychology Department, Smith College, khobbs@email.smith.edu

Chia-Chun Huang, Department of Physical Education, National Taiwan Normal University, Taipei, Taiwan.

Yoshifumi Ikeda, Department of Education for Children with Intellectual Disability, Tokyo Gakugei University, Japan, s081003p@u-gakugei.ac.jp

Makoto Inagami, Department of Built Environment, Tokyo Institute of Technology, Japan, inagami.m.aa@m.titech.ac.jp

Hiroshi Inou, DENSO Corporation, hiroshi_inou@denso.co.jp

Robert W. Isenhower, Center for the Ecological Study of Perception and Action, University of Connecticut, robert.isenhower@uconn.edu

Kiyohide Ito, Future University-Hakodate, itokiyo@fun.ac.jp

Jacob Jones, Department of Psychology, Brigham Young University

Azizah J. Jor'dan, University of Minnesota, jord0154@umn.edu 
Yu Kamiyama, Department of Education for Children with Intellectual Disability, Tokyo Gakugei University, Japan, c053113p@u-gakugei.ac.jp

So Kanazawa, Japan Women's University, Japan

Stephanie Karges, Department of Psychology, Miami University, Oxford, Ohio

Yasunobu Katsumata, Toyohashi University of Technology, Japan

Nam-Gyoon Kim, Department of Psychology, Keimyung University, nk70@kmu.ac.kr

Michiteru Kitazaki, Toyohashi University of Technology, Japan

Mitsuru Kokubun, Tokyo Gakugei University, kokubun@u-gakugei.ac.jp

Jüergen Konczak, Human Sensorimotor Control lab, School of Kinesiology, University of Minnesota, jkonczak@umn.edu

Masayuki Kumai, Tohoku University, Japan

Nikita A. Kuznetsov, University of Cincinnati, nikitakuznetsov@yahoo.com

Julien Lagarde, Motor Efficiency and Deficiency Laboratory, University Montpellier, France, julien.lagarde@univ-montp1.fr

Eric Littman, Miami University, Oxford OH, Littmaem@muohio.edu

Stacy M. Lopresti-Goodman, Center for the Ecological Study of Perception and Action, University of Connecticut, stacy.lopresti-goodman@uconn.edu

Chia-Hao Lu, Human Sensorimotor control lab, School of Kinesiology, University of Minnesota, luxxx214@umn:edu

Ludovic Marin, Motor Efficiency and Deficiency Laboratory, University Montpellier 1, ludovic.marin@univ-montp1.fr

Leonard S. Mark, Department of Psychology, Center for Ergonomic Research, Miami University, markls@muohio.edu

Kerry L. Marsh, Center for the Ecological Study of Perception and Action, University of Connecticut, kerry.l.marsh@uconn.edu

Shin Maruyama, Research Organization of Information and Systems, National Institute of Informatics, Japan, shindiana2@yahoo.co.jp 
Dawn M. McBride, Department of Psychology, Illinois State University

Hiroyuki Mishima, University of Fukui, Japan, hiromish@yb3.so-net.ne.jp

Ryo Mizuno, Graduate School of Systems Information Science Future University-Hakodate, Japan, g3107005@fun.ac.jp

Tetsushi Nonaka, University of Tokyo, nonaka.tetsushi@iii.u-tokyo.ac.jp

Ryuzo Ohno, Department of Built Environment, Tokyo Institute of Technology, Japan

Makoto Okamoto, School of System Information Science, Future UniversityHakodate, Japan

Hideyuki Okuzumi, Tokyo Gakugei University, okuzumi@u-gakugei.ac.jp

Edward W. Otten, Miami University, Oxford OH, ottenew@muohio.edu

Zsolt Palatinus, Center for the Ecological Study of Perception and Action, University of Connecticut, zsolt.palatinus@uconn.edu

Christie Pelzer, University of Minnesota

Milena Petrovic, Department of Psychology, Center for Ergonomic Research, Miami University, petrovm@muohio.edu

Amanda Phillippi, Department of Psychology, Brigham Young University

Peter B. Pufall, Psychology Department, Smith College, ppufall@smith.edu

N. Ramdani, University Montpellier II, France, ramdani@lirmm.fr

S. Ramdani, University Montpellier I, France, sofiane.ramdani@univ-montp1.fr

Veronica C. Ramenzoni, Department of P.sychology, University of Cincinnati, ramenzvc@email.uc.edu

Robert Rein, École des Hautes Études en Sciences Sociales - Groupe de Recherche Apprentissage et Contexte, Paris, France, mail@robertrein.de

Michael J. Richardson, Department of Psychology, Colby College, mjrichar@colby.edu 
Michael A. Riley, Department of Psychology, University of Cincinnati, rileym@email.uc.edu

Mamoru Sawada, Corporate R\&D Department, DENSO Corporation, Japan, mamoru_sawada@denso.co.jp

R. C. Schmidt, Department of Psychology, College of the Holy Cross, rschmidt@holycross.edu.

Keonho Shin, Department of Education, Kangnam University, Yongin, Korea

Kevin Shockley, Department of Psychology, University of Cincinnati, kevin.shockley@uc.edu

Jonathan Shook, Department of Psychology, University of Cincinnati

Paula Silva, Center for the Ecological Study of Perception and Action, University of Connecticut, paula.silva@uconn.edu

L. James Smart, Jr., Department of Psychology. Miami University, Oxford, OH,Smartlj@muohio.edu

Alison Smith, University of Minnesota

Damian G. Stephen, Center for the Ecological Study of Perception and Action, University of Connecticut, damian.stephen@uconn.edu

Thomas A. Stoffregen, University of Minnesota, tas@umn.edu

Adam Strang, Department of Psychology, Miami University, Oxford, Ohio

Masashi Takiyama, University of Tokyo, masaphy@iainukiki.jp.

Aki Tsuruhara, Department of Psychology, Chuo University, Tokyo, Japan, aki.tsuruhara@gmail.com

Michael T. Turvey, Center for the Ecological Study of Perception and Action, University of Connecticut, michael.turvey@uconn.edu

Masaki Ueno, Department of Education for Children with Intellectual Disability, Tokyo Gakugei University, Japan, c053109p@u-gakugei.ac.jp

Manuel Varlet, Efficiency and Deficiency Laboratory, University Montpellier1, Montpellier, France

Sebastien Villard, University of Minnesota, svillard@umn.edu 
Michael G. Wade, University of Minnesota, mwade@umn.edu

Jeffrey B. Wagman, Department of Psychology, Illinois State University, jbwagma@ilstu.edu

Eliah J. White, Department of Psychology, University of Cincinnati, whiteej@email.uc.edu

Melissa Wright, Department of Psychology, Brigham Young University

Masami Yamaguchi, Chuo University, Japan

Chih-Mei (Melvin) Yang, Department of Physical Education, National Taiwan Normal University, Taipei, Taiwan, melvin@ntnu.edu.tw

Lin Ye, Department of Psychology, Center for Ergonomic Research, Miami University, yel@muohio.edu

Ken Yoshida, University of Minnesota, yoshi028@umn.edu

Yawen Yu, University of Minnesota

Qin (Arthur) Zhu, Motor Learning/Control Program, Division of Kinesiology and Health, University of Wyoming, qzhu1@uwyo.edu 
Chapter 1:

Action \& Coordination 



\title{
Nonlinear Attractor Dynamics and Symmetry Breaking in Prism Adaptation and Re-adaptation
}

\author{
Julia J. C. Blau ${ }^{1}$, Damian G. Stephen ${ }^{1}$, Till D. Frank ${ }^{1}$, \\ M.T. Turvey ${ }^{1,2}, \&$ Claudia Carello ${ }^{1}$ \\ ${ }^{1}$ Center for the Ecological Study of Perception and Action, University of Connecticut, \\ USA, ${ }^{2}$ Haskins Laboratories, New Haven, CT USA.
}

The typical prism adaptation paradigm consists of three phases (Fig. 1, panel 1). In the baseline phase, participants throw to a target to establish their average natural deviation from the target. During adaptation, participants wear prism goggles that shift their gaze. The disturbed vision results in an initial systematic deviation from the target, and exponential error decay as the participant adapts to the perturbed perception-action system. The prism goggles are removed for the third phase, re-adaptation. At this point, the perception-action system is still adapted to the perturbation; which results in systematic error in the opposite direction of the original error, known as the aftereffect. This aftereffect is eliminated exponentially over the rest of the phase.

Prism Adaptation-An Extended Case. An extension of the paradigm reveals that when experimental conditions (presence of a wrist weight; FernandezRuiz et al., 2000) differ during adaptation and re-adaptation phases (symmetry breaking), then re-adaptation is only partial. Performance error (aftereffect) does decay; however, when experimental conditions are re-established (symmetry regained), performance error returns (latent aftereffect) and another readaptation process occurs.

Error Decay and the Dynamic Systems Approach. Adaptation, the error decay of the aftereffect, and the latent aftereffect all exhibit gradual, exponential error decay, rather than one-trial learning. One explanation of this phenomenon is that the system is only calibrated for fine-tuning, with a maximum step per throw (Fernandez-Ruiz \& Diaz, 2006). However, a dynamical systems approach might offer new insights. Dynamic systems theory is a powerful approach to examining perception-action systems (Beek et al., 1995; Kelso, 1995). Learning approached from this perspective has been associated with the emergence of attractors and self-organized states (Schöner \& Kelso, 1995). We propose a dynamic model for prism adaptation. 
The Model. Evolution of distance-to-target, $x(t)$, depends on the intrinsic attractor, $V_{O}$, and the adaptation attractor, $V_{A}$. The intrinsic attractor is invariant; the adaptation attractor emerges during an adaptation process and vanishes in the course of a re-adaptation process. The evolution of $x(t)$ is defined by:

$$
\frac{d}{d t} x(t)=-\frac{d}{d x} V_{0}(x)-\frac{\partial}{\partial x} V_{A}(x, t)=-\frac{\partial}{\partial x} V(x, t)
$$

where $V(x, t)$ is the total potential $V_{O}(x)+V_{A}(x, t) . V_{O}$ and $V_{A}$ can be modelled explicitly and predictions can be derived (Fig. 1, first two columns) by solving the model equation numerically and analytically (Frank, Blau, \& Turvey, 2009). For an adaptation process we studied a model equation of the form:

$$
\begin{aligned}
& \frac{d}{d t} x(t)=-b(x-s)-c \alpha(t)(x-s-\alpha(t)) \\
& \frac{d}{d t} \alpha(t)=I(x(t))
\end{aligned}
$$

a
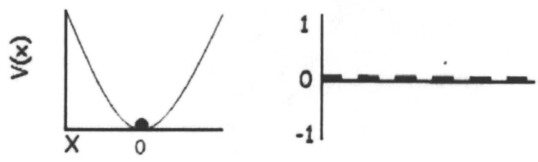

b
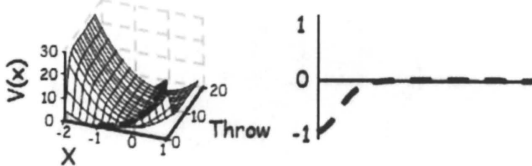

c
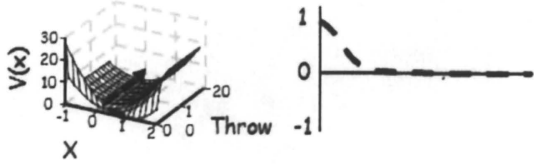

d
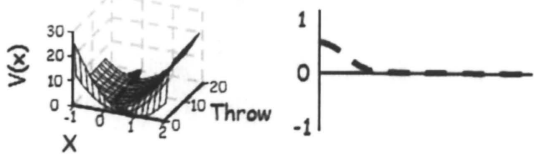

e
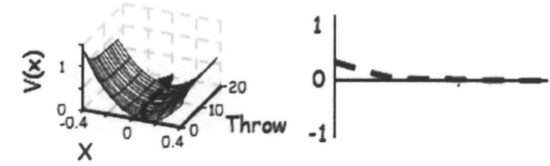
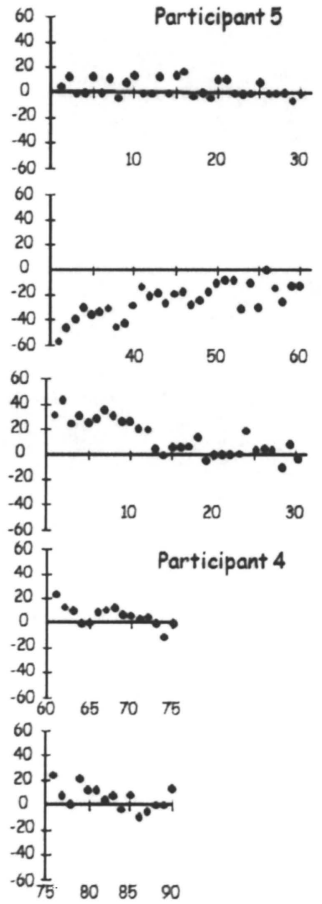

Figure 1. Explicit models (first column), predictions (second column) and examples of corresponding results (third column) in the general (panels a-c) and extended (panels $d$ and e) prism adaptation paradigm. 
where $s$ is the prismatic shift, and $\alpha$ is a time-dependent function of the adaptation potential $V_{A}$ (Fig. 1a, b). For the re-adaptation dynamics, $s=0$ (Fig. 1c). For the extended prism paradigm, we add a constant term, $\delta$, that accounts for the symmetry-breaking (Fig. 1d). For the secondary adaptation process $\delta=0$ (Fig. 1e). (See Frank, Blau, \& Turvey, 2009, for a full explication of the model).

In this model, the symmetry-breaking term, $\delta$, must be defined experimentally. Although mass has been suggested as a possibility, the mass manipulation of Fernandez-Ruiz et al. (2000) is conflated with rotational inertia. These variables are disentangled in our experiment by placing a given mass at one of two locations on the arm, thereby manipulating rotational inertia while keeping mass constant. This also offers us the opportunity to compare our model predictions to actual experimental data (Fig. 1).

\section{Method}

Forty undergraduates with normal or corrected-to-normal vision were assigned randomly to one of five groups defined by when, where, or whether prisms and weights were added or removed (Fig. 2). On each trial, a $108 \mathrm{~g}$ beanbag was thrown to a target $2.5 \mathrm{~m}$ away. Prisms (30-diopter Fresnel lenses) shifted vision to the left. A $1.15 \mathrm{~kg}$ band attached at the elbow brought about a smaller change in rotational inertia than that same band attached at the wrist.

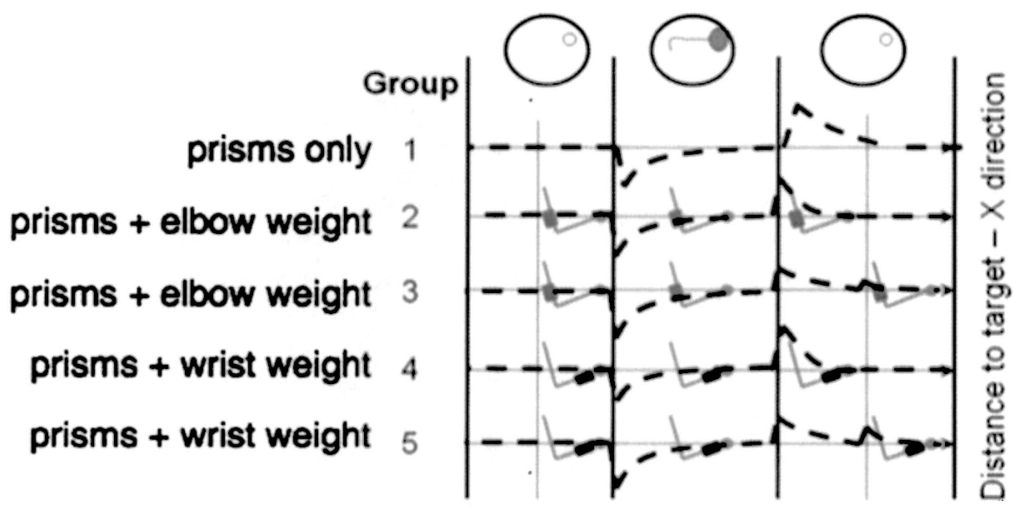

Figure 2. Vertical lines indicate when prisms and/or weights were added or removed. Results are symbolized by the dashed line. By convention, deviations below the horizontal line are to the left.

\section{Results and Discussion}

Model Fit. In all cases, the data fit the model predictions (Fig. 1, last column), suggesting that a dynamical systems model can account for gradual learning behaviour such as prism adaptation. The model was also able to account for the symmetry breaking (Fig. 1d, e).

Symmetry Breaking Term. The first aftereffect was smaller and the latent aftereffect was greater in groups 3 and 5 than in groups 2 and $4, F(1,30)=$ 
$12.82, p<.002$, confirming the existence of the latent aftereffect under conditions of symmetry breaking (Fig. 2). In Groups 1, 3, and 5, the first aftereffect declined and the latent aftereffect increased across groups, $F(2,21)=13.94, p<$ .0001 , suggesting that rotational inertia, not mass, is the variable responsible for the symmetry breaking. Further research should uncover more variables that cause such a break in an effort to discover the underlying cause (see Blau, Stephen, Carello, \& Turvey, under review, for a full discussion).

General Discussion. Prism adaptation does not exhibit one-trial learning. Prism adaptation is a dynamic process that can be described in terms of an evolution equation. Adaptation processes involve the emergence of behavioural patterns. Our modelling approach reveals that these patterns emerge in a nonlinear system. In short, our analysis suggests that adaptation requires nonlinearities. The context-dependency of prism adaptation can be understood as breaking the symmetry of conditions during adaptation and re-adaptation. Taking a dynamic systems perspective, symmetry-breaking reveals itself as an additive force. By fitting the proposed model to experimental data, meaningful parameters can be derived. Since the study of prism adaptation has clinical relevance (Brooks et al., 2007), these parameters could be used for diagnostic purposes.

\section{References}

Beek, P. J., Peper, C. E., \& Stegeman, D. F. (1995). Dynamical models of movement coordination. Human Movement Sciences, 14, 573-608.

Blau, J. J. C., Stephen, D. G., Carello, C., \& Turvey, M. T. (under review). Prism adaptation of underhand throwing: Rotational inertia and the primary and latent aftereffects.

Brooks, R. L., Nicholson, R. I., \& Fawcett, A. J. (2007). Prisms throw light on developmental disorders. Neurophysiologica, 45, 1921-1930.

Fernández-Ruiz, J., \& Díaz, R. (2006). Prism adaptation and aftereffect: Specifying the properties of a procedural memory system. Learning \& Memory, 7, 193-198.

Fernández-Ruiz, J., Hall-Haro, C., Díaz, R., Mischner, J., Vergara, P., \& LopezGarcia, J. C. (2000) Learning motor synergies makes use of information on muscular load. Learning \& Memory, 7, 193-198.

Frank, T. D., Blau, J. J. C., \& Turvey, M. T. (2009). Ninlinear attractor dynamics in the fundamental and extended prism adaptation paradigm. Physics Letters A, 373(11), 1022-1030.

Kelso, J. A. S. (1995) Dynamic patterns. MIT Press: Cambridge, MA.

Schöner, G. S. \& Kelso, J. A. S. (1988). A synergetic theory of environmentallyspecified and learned patterns of movement coordination: I. Relative phase dynamics. Biological Cybernetics, 58, 71-80.

Acknowledgments. This research was supported by National Science Foundation grant SBR 04-23036. 
Studies in Perception \& Action X

J. B. Wagman \& C. C. Pagano (Eds.)

(C) 2009 Taylor \& Francis Group, LLC

\title{
Functional Tuning of Action to Task Constraints in Tool-Use: The Case of Stone Knapping
}

\author{
Blandine Bril, Robert Rein, \& Tetsushi Nonaka
}

École des Hautes Études en Sciences Sociales, France

Tool use appears as a privileged entry to understand goal directed actions, the organism being not simply directed toward the goal, but rather directed by the goal (Shaw, 1987). The aim of the current study is to characterize the expertise in the earliest known instance of human tool-use, stone knapping, from the perspective where the emphasis is on the goal while the movement is viewed as driven by task constraints.

In stone knapping, one stone (hammer) is used to strike another (core) to remove a sharp-edged piece (flake) according to the fracture mechanism called conchoidal fracture. Counter intuitively, in conchoidal fracture, it has been demonstrated that the force of the blow does not play a major role in determining the dimension of the detached flake (Dibble \& Pelcin, 1995). It does, however, play a critical role in determining whether or not a flake can be produced in the first place. Therefore, kinetic energy of the hammer at impact is one of the essential task constraints for flake production, and we hypothesized that if actions are controlled in a functionally specific manner, knappers would maintain the kinetic energy of the hammer at impact invariant irrespective of the mass of hammers used. In other words, the achievement of the goal, the removal of a flake, is driven in such a way to meet the constraints of the task, that is, to produce the required kinetic energy of the hammer at impact of the strike.

\section{Method}

Nine participants with different knapping experience (two experts, three intermediates and four novices) participated in the experiment. Two hammers with different mass (heavy: $400 \mathrm{~g}$, light: $250 \mathrm{~g}$ ) were used. Flint weighing between 2-3 $\mathrm{kg}$ was used as cores. Striking movements were recorded using an Ascension miniBIRDC magnetic marker system set at a recording frequency of $100 \mathrm{~Hz}$. One sensor was attached to the backside of the hand. Each participant produced twenty flakes with each hammer. The trajectory length of the hammer was calculated based on the trajectory of the hand sensor in 3D space from the maxi- 
mum vertical height to the point of impact. The kinetic energy was calculated using the formula $E_{\mathrm{kin}}=1 / 2 \mathrm{~m} \mathrm{v}^{2}(\mathrm{v}=$ resultant velocity). A linear mixed-model analysis incorporating hammer weight and skill level in the fixed-effects structure and subject as a random effect was used for statistical analysis, and Bonferroni-corrected post-hoc tests were used for multiple comparisons.

\section{Results and Discussion}

590 strikes contributed to the analysis. Success rate of flaking per group was $84 \%$ for experts, $55 \%$ for intermediates, and $49 \%$ for novices.

Hammer trajectory length. Statistical analysis indicated a significant main effect for hammer weight, $\mathrm{F}(1,577)=79.1, p<.001$, as well as a significant interaction between hammer weight and skill level, $\mathrm{F}(1,577)=3.8, p<.05$. Participants used longer trajectories when striking with the lighter hammer.

Velocity at impact. Statistical analysis for striking velocity indicated a significant main effect for hammer weight, $\mathrm{F}(1,577)=52.4, p<.001$.

Kinetic energy. Statistical analysis indicated significant differences between hammer conditions, $\mathrm{F}(1,577)=14.3, p<.001$. Post-hoc test further revealed that there were no significant differences in kinetic energy between hammer conditions for experts, while the differences for intermediates and novices were both significant $(p<.01)$. All remaining effects were nonsignificant.

The results of the experiment showed that modern stone knappers adjust their movements according to task constraints. When using a lighter hammer participants across all groups increased the velocity of the hammer to achieve perceived necessary kinetic energies. However, only expert knappers were able to adjust their movement kinematics in a way which left the resulting kinetic energy unaltered between hammer conditions. In contrast, novice and intermediate skilled knappers exhibited different kinetic energies between the two conditions due to disproportional increases in striking velocity for the lighter hammer. The results indicate that knapping skill is characterized by functional tuning to task constraints. In addition, a general trend was observed where the strikes of experts tend to involve lower kinetic energy compared to novice and intermediate knappers. The fact that the success rate of detachment of flakes by strikes was highest for experts and lowest for novices implies that kinetic energy for novices was more than what was needed to detach a flake, while experts controlled their actions just sufficient to achieve the goal. The results stress the influence of perceptual parameters for the control of actions. For successful action, the actor must establish the functional relationship between himself, the tool, and the environment.

Overall, the results of this study highlight the importance of detecting the constraints of the task, and regulating actions accordingly. The characteristics of expert skills we found, fits nicely to the definition of dexterity given by Bernstein, who wrote "Dexterity is the ability to create a perfect key for any emerging lock (Bernstein, 1996, p. 215). 
Table 1. Means and standard deviations of dependent variables over all strikes

\begin{tabular}{llcccccc}
\hline \multirow{3}{*}{$\begin{array}{l}\text { Skill } \\
\text { Level }\end{array}$} & \multirow{2}{*}{ Hammer } & \multicolumn{2}{c}{$\begin{array}{c}\text { Trajectory } \\
\text { Length }(\mathrm{cm})\end{array}$} & \multicolumn{2}{c}{$\begin{array}{c}\text { Velocity } \\
(\mathrm{m} / \mathrm{s})\end{array}$} & \multicolumn{2}{c}{$\begin{array}{c}\text { Kinetic } \\
\text { Energy }(\mathrm{J})\end{array}$} \\
\cline { 2 - 8 } Expert & & $\mathrm{M}$ & $\mathrm{SD}$ & $\mathrm{M}$ & $\mathrm{SD}$ & $\mathrm{M}$ & $\mathrm{SD}$ \\
\cline { 2 - 7 } & Light & 21.91 & 6.81 & 3.39 & 0.55 & 5.91 & 2.01 \\
\cline { 2 - 8 } Intermediate & Heavy & 14.13 & 2.83 & 2.75 & 0.72 & 4.37 & 2.42 \\
\cline { 2 - 8 } & Light & 28.40 & 9.20 & 4.93 & 1.48 & 12.47 & 7.75 \\
\cline { 2 - 8 } Novice & Heavy & 27.32 & 10.87 & 4.28 & 1.51 & 11.42 & 8.04 \\
\cline { 2 - 8 } & Light & 26.67 & 5.10 & 4.10 & 1.85 & 9.45 & 10.61 \\
\hline & Heavy & 21.90 & 3.75 & 3.03 & 1.00 & 5.28 & 5.27 \\
\hline
\end{tabular}

\section{References}

Bernstein, N. A. (1996). Dexterity and Its Development. Mahwah, NJ: Lawrence Erlbaum Associates.

Dibble, H. L., \& Pelcin, A. (1995). The Effect of Hammer Mass and Velocity on Flake Mass. J. Archaeol. Sci, 22, 429-439.

Shaw, R. E. (1987). Behavior with a purpose [Review of the book GoalDirected Behavior]. Contemporary Psychology, 32, 243-245.

Acknowledgements. This research was funded by the European Union project HANDTOMOUTH. 


\title{
Variability of Uni-Manual Pendulum Oscillation at and away from Resonance
}

\author{
Dobromir G. Dotov ${ }^{1}$, Damian G. Stephen ${ }^{1}$, Till D. Frank ${ }^{1}$, \\ $\&$ M. T. Turvey ${ }^{1,2}$ \\ ${ }^{1}$ Center for the Ecological Study of Perception and Action, University of Connecticut \\ ${ }^{2}$ Haskins Laboratories
}

Extensive research based on the dual-pendulum paradigm (Kugler \& Turvey, 1987) has helped to establish overarching dynamical laws of intra- and inter-personal coordination (Amazeen, Amazeen, \& Turvey, 1998). Can the study of uni-manual pendulum oscillation too be informative about dynamic constraints on motor behavior and coordination? In order to address this question our experiment focused on the coordination between the rhythmic motor behavior of swinging a hand-held pendulum and an auditory metronome as an environmental stimulus. Previous research with the same design (using phase space reconstruction to characterize the dynamics) found that the system could be described as having fewer active degrees of freedom, less noise, and a greater prediction horizon when running at its resonance as compared to when running at lower or higher rates (Goodman, Riley, Mitra, \& Turvey, 2000). In this way, the "comfort" that the central nervous system experienced in a resonant configuration could be defined in terms of minimization of controlled variables and maximization of predictability of chaotic dynamics.

In order to extend the understanding of such a system, we delved further into the issue of variability. A vast array of research provides evidence that variability of motor behavior is not just noise extrinsic to the movement determinism but actually plays an essential role in helping the system establish its functionally appropriate behavior (for a review see, Riley \& Turvey, 2002). In this respect, Abarbanel suggests that "the range of alternatives available to which one can control a system is larger if chaotic motion, seen as a wide ranging exploration of state space, is allowed" (1996, p. vi). We can sharpen our initial question by using Abarbanel's insight as a guiding intuition. What can the properties of the oscillation variability tell us about the system? Does pushing it away from resonance mean pushing it away from its stable state, thus, inducing a potential for the system to search for alternative stable states? Is the amount and structure of variability going to express this potential? 
To begin answering these questions, we used drift-diffusion analysis based on generalized Langevin equation seeking to reconstruct the stochastic dynamics of frequency entrainment. This approach has been recommended in light of its potential ability to emphasize the intrinsic role played by the stochastic components of a dynamical system (Van Mourik, Daffertshofer, \& Beek, 2006).

On another level of analysis we focused on Detrended Fluctuation Analysis (DFA) as a means to quantify the fractal properties of the fluctuations of the wrist (Peng, Havlin, Stanley, \& Goldberger, 1995). The relation between aspects of the changing structure of variation, as expressed by power-law coefficients, and a system's moving closer or farther away from equilibrium in a statistical mechanical sense has been discussed in the literature. For example, a direct relation has been demonstrated between the fractal properties of the distribution function of a dissipative system and both its nonequilibrium steady state and relaxation towards equilibrium (Gilbert, Dórfman, \& Gaspard, 2000). Importantly, such phenomena should not be considered as isolated to physics or physical chemistry. Entropy has also been used as a predictor of the creation of new structure in the face of acquiring a novel cognitive skill (Stephen, Dixon, \& Isenhower, in press).

\section{Method}

Seven right-handed participants performed a uni-manual pendulumswinging task maintaining a steady phase relative to an auditory metronome. They received six trials in each of the three conditions of slow $(.67 \mathrm{~Hz})$, resonance $(.83 \mathrm{~Hz})$, and fast metronome $(1.33 \mathrm{~Hz})$ in a random sequence. The resonant frequency of the wrist-pendulum system was determined following the procedures outlined in Kugler and Turvey (1987) to calculate the period of the simple equivalent pendulum. A goniometer provided measures of wrist deviations (radial and ulnar). Absolute angular displacement furnished the same number of data points per condition for DFA while a peak-extracting algorithm derived trial series of rescaled cycle frequencies serving as the domain of the driftdiffusion analysis.

\section{Results and Discussion}

A repeated-measures ANOVA on the coefficient $Q$ quantifying the stochastic term in the drift-diffusion analysis of frequency entrainment found a significant effect of rate, $F(2,12)=5.77, p<.05$. A significant quadratic trend, $F(1,6)$ $=8.39, p<.05$ (Figure 1A), indicates that the noise term is minimal at resonance and increases as pendulum frequencies deviate from resonance. Similar analysis yields a significant effect for the DFA $H$ coefficient, $F(2,12)=34.23, p<.001$. As Figure 1B shows, $H$ for the fast pace was different from both resonance $(p<$ $.01)$ and slow $(p<.01)$ but they did not differ from each other $(p=.90)$. 

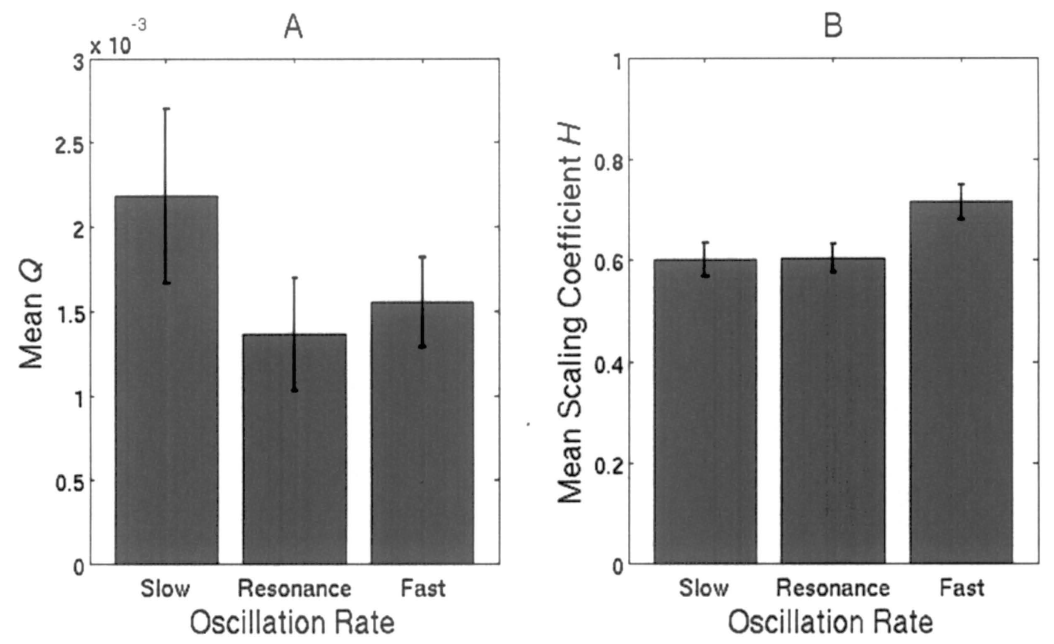

Figure 1. Means and standard error bars for the (A) frequency entrainment noise coefficient $Q$ and (B) fluctuation $H$ exponent.

At first blush, these results support our initial hypothesis that variability at non-resonance rates should be special in informative ways. At the level of frequency entrainment, which also can be defined as the amount of the behavioral cycle's deviation from target frequency, noise was augmented by deviation from resonance. A possible interpretation is that maintaining synchrony with the stimulus became harder at these rates. With regard to the noise at the level of wrist motions, the higher $H$ coefficient for fast trials, a signature of more complex fluctuations, can be interpreted in accord with the literature already discussed as indicative of a pendulum swinger being moved farther away from her equilibrium rate. Under such an interpretation, our study is congruent with the hypothesis that one route for a nonlinear system with many degrees of freedom to dissipate excessive stress is to augment its fluctuation complexity.

Our results, however, introduce some ambiguity. Setting aside the quadratic trend in the frequency entrainment noise term, there is no difference between resonance and fast paces $(p>.25)$. The pattern is reversed for mean $H$ exponents - there is no difference between slow and resonance. Why did two different patterns characterize two different levels of analysis?

A tentative explanation relies on the conception that state space below and above resonance might allow for different solutions. Drawing the stimulushuman-pendulum system away from resonance induces breaking of symmetry. In the low-rate case the broken symmetry is with respect to cycle duration whereas in the high-rate case the broken symmetry is with respect to increasing complexity of motion as expressed by an increase of the scaling coefficient. 


\section{References}

Abarbanel, H. D. I. (1996). Analysis of chaotic data. New York, NY: SpringerVerlag.

Amazeen, P. G., Amazeen, E. L., \& Turvey, M. T. (1998). Dynamics of human intersegmental coordination: Theory and research. In D. Rosenbaum \& C. Collier (Eds.), Timing of behavior: Neural, computational, and psychological perspectives. Boston, MA: MIT Press.

Gilbert, T., Dorfman, R., \& Gaspard, P. (2000). Entropy production, fractals, and relaxation to equilibrium. Physical Review Letters, 85(8), 1606-1609.

Goodman, L., Riley, M. A., Mitra, S., \& Turvey, M. T. (2000). Advantages of rhythmic movements at resonance: minimal active degrees of freedom, minimal noise, and maximal predictability. Journal of Motor Behavior, 32(1), 3-8.

Kugler, P. N., \& Turvey, M. T. (1987). Information, natural law, and the selfassembly of rhythmic movements. Hillside, NJ: Erlbaum.

Peng, C.-K., Havlin, S., Stanley, H. E., \& Goldberger, A. L. (1995). Quantification of scaling exponentials and crossover phenomena in nonstationary heartbeat time series. Chaos, 5(1), 82-87.

Riley, M. A., \& Turvey, M. T. (2002). Variability of determinism in motor behavior. Journal of Motor Behavior, 34(2), 99-125.

Stephen, D. G., Dixon, J. A., \& Isenhower, R. W. (in press). Dynamics of representational change: Entropy, action, and cognition. Journal of Experimental Psychology: Human Perception and Performance.

van Mourik, A. M., Daffertshofer, A., \& Beek, P. J. (2006). Deterministic and stochastic features of rhythmic human movement. Biological Cybernetics, 94(3), 233-244.

Acknowledgments. Preparation of this manuscript was supported by a grant from the University of Connecticut Research Foundation. 


\title{
Comparison of Performance of Tray-Carrying Task by Persons with Down Syndrome and Those With Other Forms of Mental Retardation
}

\author{
Shogo Hirata ${ }^{1}$, Hideyuki Okuzumi ${ }^{1}$, Mitsuru Kokubun ${ }^{1}$ \\ \& Masayuki Kumai ${ }^{2}$ \\ ${ }^{1}$ Tokyo Gakugei University ${ }^{2}$ Tohoku University
}

This study compares the performance of a tray-carrying task by persons with Down syndrome and those with other forms of mental retardation, and clarifies the features of motor skills execution in persons with Down syndrome. The tray-carrying task is a motor task devised by Kokubun (1999). This task requires subjects to carry a tray bearing a glass filled with water as fast as possible over a distance of 3 meters, but without spilling the water. The time taken and amount of water spilled are measured. Kokubun (1999) compared performance of this task by children with Down syndrome and by children with other forms of mental retardation. The results showed that children with Down syndrome were indeed slower than children with other types of mental retardation, but the amount of water spilled was no different between the two groups. That is to say, while persons with Down syndrome were slow, they were not inaccurate. Although the above findings of Kokubun (1999) have a great impact in changing the general view that persons with Down syndrome were 'clumsy', few studies have been conducted from this perspective. In this study, we conducted the same motor task to confirm the findings of Kokubun (1999), and we conducted a normal walking task to compare to the performance of the tray-carrying task.

\section{Method}

Thirteen persons with Down syndrome ( 7 males, 6 females) aged 14 to 48 years $(29 \pm 11.8$ years) and 13 persons with other forms of mental retardation (6 males, 7 females) aged 13 to 48 years ( $29 \pm 11.0$ years) without sensory and motor impairments were used as subjects. In this study, subjects with Down syndrome were diagnosed by medical doctors, and no one with Autism was included in the group with other forms of mental retardation.

For the tray-carrying task, subjects were given a tray with a glass $(9 \mathrm{~cm}$ height, $7 \mathrm{~cm}$ diameter at the top, $4.5 \mathrm{~cm}$ at the bottom, and a capacity of $225 \mathrm{ml}$ ) filled with of $200 \mathrm{ml}$ water at the start line and instructed to carry it to a goal line $3 \mathrm{~m}$ away as fast as they could without spilling it. The time to reach the goal line was measured with a stop watch. The amount of water remaining in the glass on 
completion of the task was measured with a measuring cylinder, and this value was subtracted from $200(\mathrm{ml})$ to calculate the amount of water spilled. The average of two trials was used as the representative value for that subject. In normal walking, subjects were requested to walk normally for $3 \mathrm{~m}$ to compare to the performance of the tray-carrying task. The time was measured. In addition, the adjustment ratio (the time of the tray-carrying task divided by that of normal walking) was also calculated.

\section{Results and Discussion}

Table 1 summarizes the mean (M) and standard deviation (SD) of each of the measures for both groups.

Table 1 . Tray-carrying performance by group

\begin{tabular}{|c|c|c|c|c|c|c|c|c|}
\hline & \multicolumn{4}{|c|}{ Tray-carrying task } & \multirow{2}{*}{\multicolumn{2}{|c|}{$\begin{array}{l}\text { Normal } \\
\text { walking, sec }\end{array}$}} & \multirow{2}{*}{\multicolumn{2}{|c|}{$\begin{array}{l}\text { Adjustment } \\
\text { ratio }\end{array}$}} \\
\hline & \multicolumn{2}{|c|}{ Time, sec } & \multicolumn{2}{|c|}{$\begin{array}{l}\text { Amount of } \\
\text { water spilled, } \mathrm{ml}\end{array}$} & & & & \\
\hline & $\mathrm{M}$ & SD & $M$ & SD & $\mathrm{M}$ & SD & M & SD \\
\hline $\begin{array}{l}\text { Down } \\
\text { Syndrome }\end{array}$ & 7.98 & 4.04 & 0.08 & 0.28 & 4.26 & 1.21 & 1.97 & 1.14 \\
\hline $\begin{array}{l}\text { Mental } \\
\text { retardation }\end{array}$ & 6.54 & 2.53 & 1.08 & 1.55 & 4.60 & 2.27 & 1.52 & 0.43 \\
\hline
\end{tabular}

Persons with Down syndrome took more time to complete the task than those with other forms of mental retardation. However, the time required for the normal walking task did not differ between the groups. Persons with Down syndrome spilled less water than the others. Although the adjustment ratio of persons with Down syndrome was larger than that of the others, a 2 (Subject: Down Syndrome vs. Mental Retardation) $\times 2$ (Task condition: tray-carrying vs. normal walking) analysis of variance (ANOVA) yielded no significant differences between the groups $\left(\mathrm{F}_{1,24}=0.40, \mathrm{p}>.05\right)$, but did yield significant differences between the task conditions $\left(\mathrm{F}_{1,24}=21.97, \mathrm{p}<.05\right)$. The interaction was not significant $\left(\mathrm{F}_{1,24}=2.16, \mathrm{p}>.05\right)$. In the amount of water spilled and adjustment ratio, a one-way (subject group) ANOVA yielded significant differences only in the spillage (water: $\mathrm{F}_{1,24}=5.23, \mathrm{p}<.05$. ratio: $\mathrm{F}_{1,24}=1.61, \mathrm{p}>.05$ ).

In normal walking, persons with Down syndrome perform at the same speed as those with other forms of mental retardation. But, in the tray-carrying task, persons with Down syndrome tend to take more time than for their normal walking, and behave more cautiously and accurately than people with other forms of retardation. These results accord with the findings of Kokubun (1999). In recent years, Latash (2008) suggested that persons with Down syndrome do not have major qualitative deficits in their motor control mechanisms, and that their clumsy movement was a product of an adaptive strategy to compensate for their delayed decision-making or cerebellar dysfunction. His views are consistent with the results in our study. Bernstein (1996) acutely pointed out 'a de- 
mand for dexterity is not in the movements themselves but in the surrounding conditions.' In further investigation, we would like to examine the ecological reality of 'tray-carrying' for persons with Down syndrome and those with other forms of mental retardation using various task conditions (i.e., the size of the glass or amount of water were changed). In our short study, we cannot consider the effect of the subject's intelligence level on task performance. We will address this issue in another article.

\section{References}

Bernstein, N. A. (1966). On Dexterity and Its Development. Mahwah, NJ: Lawrence Erlbaum Associates.

Kokubun, M. (1999). Are children with Down syndrome less careful in performling a tray-carrying task than children with other types of mental retardation? Perceptual and Motor Skills, 88, 1173-1176.

Latash, M. L. (2008). Neurophysiological Basis of Movement (Second Edition). Champain, IL: Human Kinetics.

Acknowledgements. The authors would like to thank the participants who made this work possible. 


\title{
Observation of Action Slips in a Young Child
}

\author{
Naoya Hirose
}

\author{
Kyoto Notre Dame University
}

This study investigated a child's action slips during acquisition of a new daily skill. Action slips are the errors that occur when people do an action that is not intended. This study differs in two respects from previous studies on action slips (e.g., Norman, 1981; Reason, 1984). One difference is that we observed a young child instead of adults. Most studies on action slips have been conducted with adults, and little attention has been devoted to children's slips up to now. Developmental research is needed to elucidate the nature of action slips. To the best of our knowledge, this is the first study to report the child's action slips in a daily setting.

The other difference from previous studies is that whereas most studies on action slips have employed the diary method, this study did not. There are several difficulties in the diary method. For example, Reason (1984) argued that, in diary studies, there are at least three kinds of biases (volunteer bias, selection bias, and recording bias). In addition, it is almost impossible for children to keep diaries for their action slips. Thereby, we used video recordings instead of diaries.

It is often pointed out that diary studies underestimate the frequency of slips. People often miss writing down slight slips in their diary, whereas video recoding method can record all of them. Thus, the method can obtain a more accurate count of the frequency of slips. Additionally, it can eliminate the selection and recording biases.

In this study, we coded microslips as well as action slips. Microslips are subtle miscues that occur in manipulation tasks (Reed, Palmer, \& Schoenherr, 2009). We identified action slips and microslips according to Hirose's (2007) coding scheme. We first defined the basic units of action and described sequences of action by using these units. Then we identified slips as deviations from the normal sequence, and discriminated between action slips and microlips. Action slips are deviations across multiple basic units, whereas microslips are within one basic unit. Consequently, our definition of slips is rather wider than the previous studies on action slips.

To explore the developmental nature of action slips, we observed a child's mouth rinsing activity during toothbrushing. We chose this behavior because we found frequent action slips in the preliminary observations. In addition, the recording was easy to accomplish because the activity occurred in the same place at around the same time. 


\section{Method}

The only participant of this study was a 4-year-old girl. Her mouth rinsing activities during toothbrushing after dinner were observed for 4 months (from 4:0 to 4:4). Her daily routine of toothbrushing was as follows: First, she brushed her teeth by herself. Second, her parent thoroughly brushed them again. Finally, she rinsed her mouth by herself. She was able to rinse her mouth by herself a few weeks before the time when the study began.

In order to record her mouth rinsing activities, a network camera (AXIS $207 \mathrm{~W}$ ) was installed at the side of a washstand in her house. Video recordings were done automatically by using the motion detection function built in the recording system. All video files were saved to a PC connected to the network. Video files including the participant's mouth rinsing activities were selected to be analyzed. The recording system was always in operation through the period of study, but the target activities were occasionally not recorded due to malfunction of the system or the participant's absence from home. Thus, we obtained 95 day's video files for analysis.

\section{Results and Discussion}

In the first analysis, we derived a "modal route" (Joe, Ferraro, \& Schwartz, 2002) of the mouth rinsing activity without any slips. The route was as follows: ascend step; take cup; hold cup under tap; turn on tap; turn off tap; sip water; swish water around in mouth; spit water; empty cup; put down cup; descend step; wipe mouth with towel. We identified any deviations from this route as slips. Then, we divided these slips into action slips and microslips according to the aforementioned criteria. A series of slips was counted as one action slip or microslip, and those slips that included both action slips and microslips were coded as one action slip. The analysis yielded a total of 62 slips.

Next, we classified slips into five types according to the previous studies of action slips. However, some microslips that could not be categorized into these types were labeled as others. Results of this classification are shown in Table 1. Captures were the most frequent type, accounting for $45.2 \%$ of all slips.

Capture. Another activity may capture control of the intended activity. The participant often veered from the hand washing activity.

Redoing. Repeated actions that need no repetition in the normal situation. The participant turned on the tap twice.

Anticipation. Skip a necessary action and perform the next step. The participant turned on the tap before holding the cup under it.

Sequential. Sequential errors other than anticipations. The participant attempted to pick up the cup in an inappropriate situation.

Hesitation. Common microslips including small pauses during movement. The participant suddenly stopped reaching for the cup, and then resumed reaching. 
Finally, we examined developmental changes in slips by dividing the period of study into halves (Figure 1). As shown in Figure 1, action slips decreased drastically in the second half. This suggests that the participant rapidly acquired the skill of mouth rinsing. In contrast, microslips decreased slightly, suggesting that microslips may continue through the time period.

In concluding, we should note that diary studies substantially underestimate the frequency of action slips, estimating one per day at most (Jonsdottir, Adolfsdottir, Cortez, Gunnarsdottir, \& Gustafsdottir, 2007). It seems surprising that, in this study, we found a number of slips during a relatively brief activity. Diary method reveals cognition after action, whereas video recording method reveals cognition in action. Future research on slips should focus on cognition in action (Reed, Montgomery, Palmer, \& Pittenger, 1995).

Table 1. Frequencies of different types of action slips and microslips.

\begin{tabular}{lcc}
\hline Type & Action Slips & Microslips \\
\hline Capture & 6 & 22 \\
Redoing & 5 & 4 \\
Anticipation & 3 & 4 \\
Sequential & 1 & 4 \\
Hesitation & 0 & 5 \\
Other & 1 & 7 \\
\hline Total & 16 & 46 \\
\hline
\end{tabular}

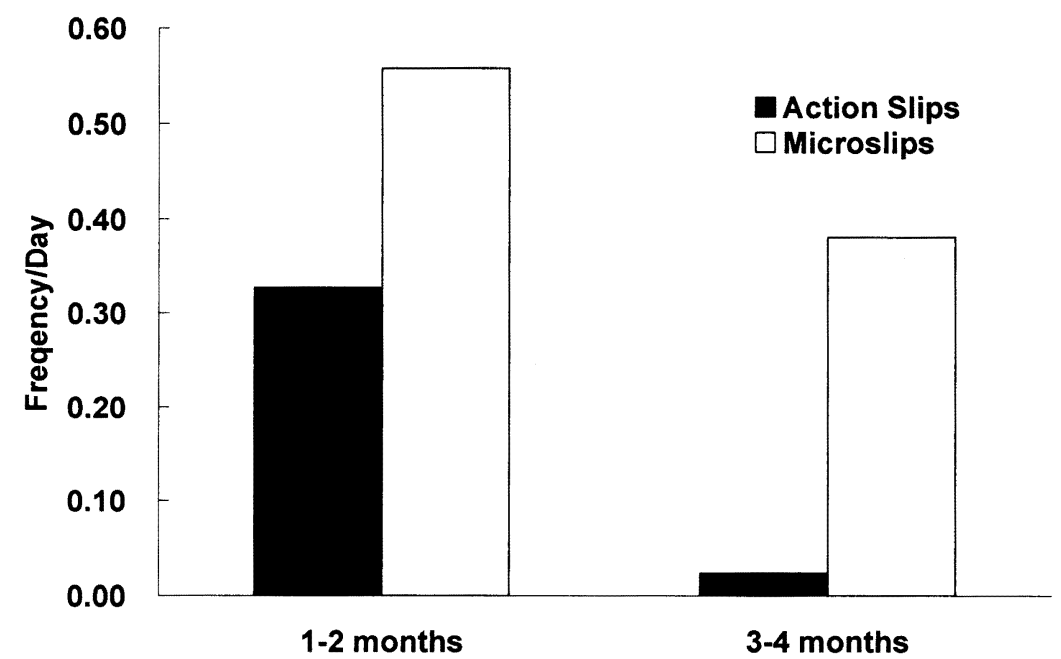
study.

Figure 1. Changes in frequency of slip occurrence per day during the period of 


\section{References}

Hirose, N. (2007). Towards a new taxonomy of microslips. In S. CumminsSebree, M. A. Riley \& K. Shockley (Eds.), Studies in perception and action $I X$. (pp. 91-94). Mahwah, NJ: Lawrence Erlbaum Associates.

Joe, W. Q., Ferraro, M., \& Schwartz, M. F. (2002). Sequencing and interleaving in routine action production. Neurocase, 8, 135-150.

Jonsdottir, M. K., Adolfsdottir, S., Cortez, R. D., Gunnarsdottir, M., \& Gustafsdottir, A. H. (2007). A diary study of action slips in healthy individuals. Clinical Neuropsychologist, 21, 875-883.

Norman, D. A. (1981). Categorization of action slips. Psychological Review, $88,1-15$.

Reason, J. T. (1984). Lapses of attention in everyday life. In R. Parasuraman \& D. R. Davies (Eds.), Varieties of attention (pp. 515-549). Orlando, FL: Academic Press.

Reed, E. S., Montgomery, M., Palmer, C., \& Pittenger, J. (1995). Method for studying the invariant knowledge structure of action: Conceptual organization of an everyday action. American Journal of Psychology, 108, 37-65.

Reed, E. S., Palmer, C. F., \& Schoenherr, D. (2009). On the nature and significance of microslips in everyday activities. Journal of Ecological Psychology, 4, 51-66. 


\title{
Relationship Between Attention and Stepping
}

\author{
Yoshifumi Ikeda, Shogo Hirata, Hideyuki Okuzumi, \\ \& Mitsuru Kokubun
}

Tokyo Gakugei University

\begin{abstract}
Attention when walking was examined in a study that measured reaction time (RT) on a probe-reaction task performed while walking on a treadmill (Kurosawa, 1994). This study concluded that the speed of treadmill walking at which RT minimized approximated the speed of a preferred gait, and that the faster or slower the walking speed, the longer RT became. According to a dynamical systems approach (Hoyt \&Taylor, 1981), the stability of system coordination is higher in the optimal mode of walking, that is, the burden of a preferred gait on attention is essentially lower than any other speed of walking. It is, therefore, well supported that RT on a probe-reaction task could minimize at a preferred gait in the study by Kurosawa (1994). Although this finding is quite interesting, there are few other studies that concluded similarly. Therefore, this study examines stepping, an analogy of walking, and the relation between the stability of the motor system and attention by assigning a probe-reaction task and the Stroop test (Stroop, 1935) while in synchronized stepping under various rhythm conditions.
\end{abstract}

\section{Method}

A total of 10 adults (4 male, 6 female) from 17 to 29 years of age ( $22.90 \pm 2.88$ years) participated in this study. Subjects were to perform a probereaction task and the Stroop test, which are cognitive tasks to assess attention, while in synchronized stepping under five rhythm conditions of $0.5 \mathrm{~Hz}, 1.0 \mathrm{~Hz}$, $2.0 \mathrm{~Hz}, 3.0 \mathrm{~Hz}$, and $4.0 \mathrm{~Hz}$ at a fixed place. A metronome was used to make sounds informing subjects of the rhythms. In a probe-reaction (Probe) task which used black rectangular patches as stimuli, subjects named the color of the black patches presented sequentially. The Stroop test was composed of two tasks: a color naming $(\mathrm{CN})$ task, in which subjects named the color of patches in red, blue, green, or yellow presented in a random order; an incongruent color naming (ICN) task, in which subjects named the color of words printed in incongruent colors presented in a random order. In ICN task, stimuli were four 
words ('red', 'blue', 'yellow', and 'green') printed in a non-matching color of the same four colors. All the stimuli of Probe, $\mathrm{CN}$, and ICN tasks were presented in the white-screened monitor in front of subjects and the reaction time $(\mathrm{msec})$ between presentations of stimuli and oral responses in a microphone was measured by Super Lab (Cedrus $\left({ }^{\circledR}\right)$. The mean time of 8 trials for Probe, $\mathrm{CN}$, and ICN tasks in each stepping rhythm condition were used as representative subject variables.

\section{Results and Discussion}

Figure 1 shows the mean of RT on Probe, $\mathrm{CN}$, and ICN tasks for each stepping rhythm condition. Figure 2 shows logarithmic $\mathrm{Hz}$ and $\mathrm{RT}$ placed on the horizontal axis and vertical axis, respectively. In both figures, the decisive coefficient of the retrogressive curve was broadly high in Probe, $\mathrm{CN}$, and ICN tasks. For Probe, $\mathrm{CN}$, and ICN tasks, the RT minimized at $2.0 \mathrm{~Hz}$ and extended whether the $\mathrm{Hz}$ was higher or lower. Therefore, in any cognitive tasks, the change of RT between each rhythm condition showed a $U$-shaped curve whose trough was around $2.0 \mathrm{~Hz}$. In other words, the low burden on attention occurred when stepping around $2.0 \mathrm{~Hz}$. Since it has been already clarified that changes of height and the interval of stepping while performing minimize when stepping around $2.0 \mathrm{~Hz}$ (Kamiyama et al; submitted), the coordination and stability of stepping at $2.0 \mathrm{~Hz}$ can be even higher. Thus, it follows that a similar conclusion to Kurosawa (1994) applies for stepping as well.

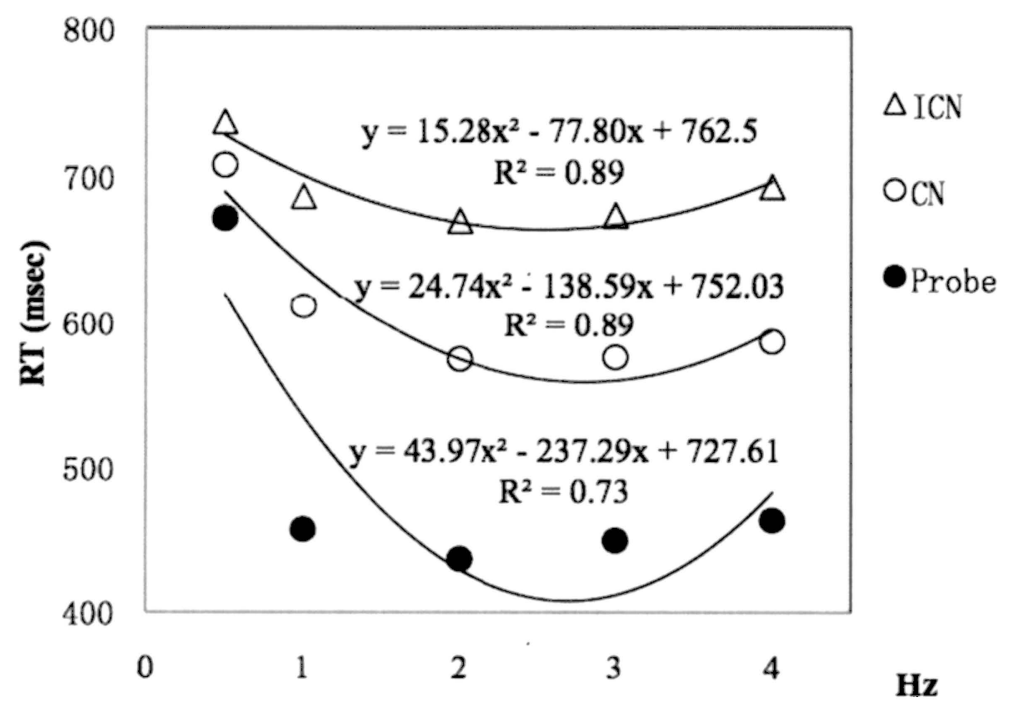

Figure 1. Mean reaction time of cognitive tasks at each stepping rhythm. 
A two-way ANOVA (within subjects) demonstrated significant effects of the cognitive task-factor $\left(\mathrm{F}_{2,18}=65.990, \mathrm{p}<.001\right)$, the stepping rhythm-factor $\left(\mathrm{F}_{4,36}=44.749, \mathrm{p}<.001\right)$ and interaction effects between the cognitive task-factor and the stepping rhythm-factor $\left(\mathrm{F}_{8,72}=4.793, \mathrm{p}<.001\right)$. The significant interaction effects suggest that the effect of rhythm conditions will not be uniform for each cognitive task. Apparent from the relation between cognitive tasks, RT on the Probe task was shorter than RT on the $\mathrm{CN}$ task, and RT on the $\mathrm{CN}$ task was shorter than the ICN task in any rhythm condition, which would be affected by the difference in difficulty. However, the change of RT between rhythm conditions in the ICN task, which is thought to be the most difficult cognitive task, was less distinct than that of the other two tasks. That is, the ICN task in the Stroop test has a constant difficulty as a cognitive task irrespective of the stability of stepping. This is more than a little paradoxical considering the greater changes in RT between rhythm conditions in the Probe and CN tasks, which are thought to be less difficult. On the other hand, from figure 1, the extension of RT was not uniform whether it was in the higher or lower $\mathrm{Hz}$ with $2.0 \mathrm{~Hz}$ centered. This suggests that the burdens of stepping at higher and lower $\mathrm{Hz}$ are different from one another. The relation between the stability of stepping and the performance on cognitive tasks has to be discussed in more detail in another article.

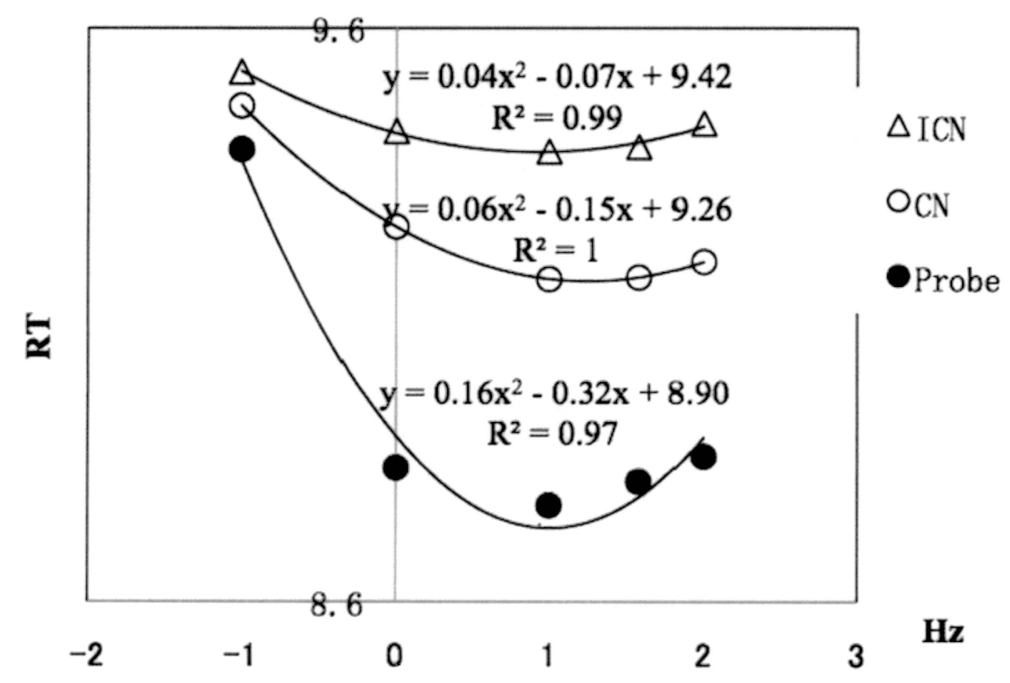

Figure 2. Mean reaction time of cognitive tasks at each stepping rhythm (Logarithm). 


\section{References}

Hoyt, D. \& Taylor, C. (1981). Gait and the energetics of locomotion in horses. Nature, 292, 239-240.

Kurosawa, K. (1994). Evaluation of locomotion efficiency by Probe-reaction time during treadmill walking at various speed and cadence. Journal of Kyorin Medical Society, 25, 527-536. (In Japanese with English abstract.) Stroop, J. R. (1935). Studies of interference in serial verbal reactions. Journal of Experimental Psychology, 18, 643-662.

Acknowledgements. The authors would like to thank the participants who made this work possible. 


\title{
Relationship Between Stepping and its Tempo
}

\author{
Yu Kamiyama, Shogo Hirata, Hideyuki Okuzumi, \\ \& Mitsuru Kokubun
}

Tokyo Gakugei University

In recent years, many studies of reciprocal movement have been conducted using the dynamical systems approach (Kelso, 1995). There are many kinds of reciprocal movements. Sekiya et al, (1996) focused on the human gait, which is a representative reciprocal movement, and clarified that whether the walking speed is fast or slow, the variability of elements, such as increasing step length or step rate, may increase and lead to a breakdown of gait stability. Although the above findings of Sekiya et al, (1996) are very interesting, few studies have been conducted on reciprocal movements from this viewpoint. In this study, we investigate the relationship between stepping and its tempo. A synchronized stepping movement is a similar movement to the human gait.

\section{Method}

The subjects were 30 healthy adults ( 15 males, 15 females) aged 20 to 29 years (22.81 \pm 1.70 years). In this study, the subjects stepping movement was recorded by a position sensor (C2399, Hamamatsu Photonics, Japan), consisting of a camera and amplifier which could record the track trajectory of an LED from a distance. The track of the LED is output laterally and sagittally. These two analog signals were input to a personal computer though an $A / D$ conversion board at a sampling frequency of $100 \mathrm{~Hz}$ and recorded on a hard disk. Subjects stand up on a flat floor wearing low-heeled shoes or thin socks and a right ankle band to which the LED was attached, and were asked to step according to a sound. Sound stimuli were presented by electronic metronome, and the sounds continued while stepping. In this study, we presented five rhythm tempos such as $0.5 \mathrm{~Hz}, 1 \mathrm{~Hz}, 2 \mathrm{~Hz}, 3 \mathrm{~Hz}$ and $4 \mathrm{~Hz}$, which were randomized for each person. Finally, subjects were asked to step naturally without the rhythm sounds at a preferred tempo.

Using the recorded digital data, we calculated the ten values of step time (ms) and step height $(\mathrm{cm})$ at each rhythm tempo. Then, the average values (step time, step height) and coefficients of variation (time CV, height CV) were calculated for each person. Step time is defined as the average rhythm in the stepping movement, and can be used as index of whether the subject stepping is synchronized with the presented stimulus. Step height is an index that shows the maxi- 
mum movement amplitude in the vertical direction in right leg. The time $\mathrm{CV}$ and height CV can be used as an index of step time variability and step height variability. If these two values are large, the variability of step time or step height is high, and the stability of stepping is low.

\section{Results and Discussion}

Table 1 summarizes the mean (M) and standard deviation (SD) of each measurement at each rhythm tempo. In this study, we only measure the right leg movement by the position of the sensor. Thus, the step time's theoretical value was the time for two iterations of stimulus sound at the each rhythm tempo, such as $0.5 \mathrm{~Hz}=4000 \mathrm{~ms}, 1 \mathrm{~Hz}=2000 \mathrm{~ms}, 2 \mathrm{~Hz}=1000 \mathrm{~ms}, 3 \mathrm{~Hz}=667 \mathrm{~ms}$, and 4 $\mathrm{Hz}=500 \mathrm{~ms}$.

Table 1. Mean (M) and standard deviation (SD) of each variable at each rhythm tempo

\begin{tabular}{lllllllll}
\hline Rhythm & \multicolumn{2}{c}{ Step time (ms) } & \multicolumn{3}{c}{ Step height (cm) } & \multicolumn{2}{c}{ Time CV } & \multicolumn{2}{c}{ Height CV } \\
tempo & $\mathrm{M}$ & $\mathrm{SD}$ & $\mathrm{M}$ & $\mathrm{SD}$ & $\mathrm{M}$ & $\mathrm{SD}$ & $\mathrm{M}$ & $\mathrm{SD}$ \\
\hline $0.5 \mathrm{~Hz}$ & 4001.17 & 26.09 & 14.78 & 7.17 & 0.05 & 0.02 & 0.18 & 0.11 \\
$1 \mathrm{~Hz}$ & 1994.83 & 12.83 & 14.51 & 6.48 & 0.04 & 0.02 & 0.14 & 0.05 \\
$2 \mathrm{~Hz}$ & 1000.00 & 5.25 & 9.17 & 4.42 & 0.04 & 0.01 & 0.12 & 0.04 \\
$3 \mathrm{~Hz}$ & 668.50 & 7.67 & 5.63 & 2.85 & 0.06 & 0.02 & 0.15 & 0.05 \\
$4 \mathrm{~Hz}$ & 505.67 & 7.76 & 4.63 & 2.23 & 0.08 & 0.02 & 0.17 & 0.06 \\
\hline Prefer & 1107.00 & 188.82 & 10.12 & 5.46 & 0.04 & 0.02 & 0.11 & 0.05 \\
\hline
\end{tabular}

The results show that the step times correspond to the theoretical value at each rhythm tempo. Moreover, the preferred tempo approximates the values at 2 $\mathrm{Hz}$. Step height rises significantly with the slowing of the tempo $\left(F_{5,145}=54.2, p\right.$ $<.01)$. Time CV becomes larger when the tempo is slower or faster, and it is a minimum at $1 \mathrm{~Hz}$ and $2 \mathrm{~Hz}\left(F_{5,145}=27.7, p<.01\right)$. The height $\mathrm{CV}$ also becomes larger when the tempo is slower or faster, and it is a minimum at $2 \mathrm{~Hz}\left(F_{5,145}=\right.$ $7.3, p<.01)$. The change of each $\mathrm{CV}$ between each rhythm tempo formed a Ushaped curve whose trough was around $2 \mathrm{~Hz}$. Each $\mathrm{CV}$ of the preferred tempo was small, and the stability of preferred stepping was very high.

Results in this study were as follows. First, step time strongly correlates to step height. This relationship between step time and step height suggests the possibility that one synergy (Bernstein, 1996) was formed in stepping. Second, in the vicinity of $2 \mathrm{~Hz}$ tempo, temporal stepping stability and spatial stepping stability were very high, whereas, when the rhythm tempo became faster or slow than $2 \mathrm{~Hz}$, there was a breakdown of stepping stability. These results accord with the findings of Sekiya et al. (1996). In this study, we could say that the stability of preferred stepping was very high. In further studies, we will have to examine various-aged children's performance to investigate the developmental process of stability of stepping. 


\section{References}

Bernstein, N. A. (1966). On Dexterity and Its Development. Mahwah, NJ: Lawrence Erlbaum Associates.

Kelso, J. A. S. (1995). Dynamic Pattens. Cambridge, MA: The MIT press.

Sekiya, N., Nagasaki, H., Ito, H., Furuna, T (1996). The invariant relationship between step length and step rate during free walking, Journal of Human Movement Studies, 30, 241-257. (In Japanese with English abstract.)

Acknowledgements. The authors would like to thank the participants who made this work possible. 
Studies in Perception \& Action X

J. B. Wagman \& C. C. Pagano (Eds.)

(C) 2009 Taylor \& Francis Group, LLC

\title{
Dynamics of Motor Performance in Visually Guided Force Production
}

\author{
Nikita A. Kuznetsov \& Michael A. Riley \\ Perceptual-Motor Dynamics Laboratory, Department of Psychology, \\ University of Cincinnati
}

The structure of motor variability is informative about underlying motor control processes (Riley \& Turvey, 2002; Slifkin \& Newell, 1999). The presence of structured (non-random) fluctuations in movement is thought to indicate a capacity for greater behavioral flexibility and greater overall adaptability to novel situations compared to systems that do not exhibit this pattern of fluctuation. In this study we examined the structure of variability of single-digit force production in regard to the use of adaptive motor control strategies.

Producing a specified level of force could be achieved using two general strategies. The first involves anticipatory modulations of force in order to keep current performance within criterion levels. This is possible if the frequency of visual feedback is high and the task goal is known, conditions that are known to facilitate performance (Sosnoff \& Newell, 2005). The second strategy involves utilizing feedback in a reactive, rather than prospective, fashion-force corrections are made only after the force criterion is violated. This strategy is expected when information that permits prospective control is absent.

The goal of this study was to determine the relation of the structure of force fluctuations to prospective and reactive force control strategies. Participants produced a specified force under manipulations of visual information about performance. A continuous display, which was designed to encourage prospective control, indicated the current level of force in relation to the goal and to the boundaries of an acceptable range around the goal. A discrete feedback display, which was designed to elicit a reactive strategy, only signalled when (and in which direction) the current level of force deviated from the acceptable range. Prospective control in the continuous condition was expected to yield smoother changes in force values. In contrast, the reactive changes in force based on discrete feedback were expected to yield discontinuous force time series containing occasional irregularities due to rapid force readjustments enacted when feedback was presented. These structural differences in the force signals were quantified using the \%LAM measure from recurrence quantification analysis (RQA; Marwan, 2002; Weber \& Zbilut, 2005). \%LAM is the percentage of recurrent points 
forming vertical lines in the recurrence plot. Signals with more discontinuities exhibit a greater number of laminar states (and hence greater $\% L A M$ ) than more smooth, continuous signals.

\section{Method}

Eleven right-handed University of Cincinnati undergraduates participated for course credit. Participants attempted to produce a constant level of force $(20 \%$ of the participant's maximum; mean maximum force was $37.1 \pm 8.7 \mathrm{~N}$ ) with two different kinds of displays (continuous vs. discrete) and three levels of difficulty (allowed force variability of 1,5 , and $10 \%$ of the target level). There were 4 trials in each condition ( 24 trials total), each lasting 30 seconds, presented in random order. Force was recorded at $100 \mathrm{~Hz}$ using a Bertec (Worthington, $\mathrm{OH}$ ) load cell. Unfiltered forces normal to the load cell surface were analyzed.

Participants sat at a table with the load cell mounted on it, $40 \mathrm{~cm}$ away from a monitor positioned at eye height. In the continuous display condition, the goal force was indicated by a gray band on the display spanning the minimum and maximum levels of allowed force. Current force was indicated in real time by a red dot. Participants tried to maintain the dot within the band. They could always see how the force they were producing related to the goal level.

In the discrete display condition participants were asked to produce the same levels of force, but the red dot only took three possible discrete, static values: above, within, or below the band, indicating, respectively, that force exceeded, matched (within limits), or was less than the goal force. Force was measured in real time but the dot position did not change unless force changed from one of those three states to another.

RQA was conducted using $d=3, \tau=20$, and a variable radius calculated as a percentage of the mean distance between time-delayed vectors of force values. Radius was adjusted to achieve $\% R E C=1 \pm 0.2$ for each trial. Two consecutive vertical points defined a vertical line. The first 4 seconds of the trial were discarded to omit transients.

\section{Results and Discussion}

Figure 1 shows sample force time series and corresponding recurrence plots. The percentage of sampled force values within the allowed limits (accuracy) and \%LAM were calculated for each trial and averaged over trials in each condition. These averaged values were submitted to separate 2 (display: continuous vs. discrete) $\times 3$ (difficulty: 1,5 , and $10 \%$ of target force level) repeatedmeasures ANOVAs.

There was a main effect of difficulty on accuracy, $F(2,22)=990.76, p<$ $.0001, \eta_{p}{ }^{2}=.99$. Participants were least successful in the $1 \%$ condition followed by the $5 \%$ and $10 \%$ conditions (Figure 2, left). Participants performed better with the continuous displays than the discrete ones, $F(1,22)=49.32, p<.0001$, $\eta_{p}{ }^{2}=.82$ - it was harder to maintain the required level of force using discrete 
feedback. The difficulty $\times$ display interaction approached but did not reach significance, $F(2,22)=3.34, p=.054, \eta_{p}{ }^{2}=.23$.

Discrete displays led to higher $\% L A M$ than continuous displays, $F(1,22)=$ $102.68, p<.0001, \eta_{p}{ }^{2}=.90$ (Figure 2, right). This indicates a greater tendency for discontinuous irregularities in the force time series in the discrete display condition, and suggests that $\% L A M$ can be used to measure differences between the qualitatively different motor control strategies encouraged by these display conditions. Difficulty did not have an effect on $\% L A M,\left(p=.09, \eta_{p}{ }^{2}=.20\right)$, and although differences between the two kinds of display conditions were more pronounced in the most difficult ( $1 \%$ range) condition there was no difficulty $x$ display type interaction $\left(p=.42, \eta_{p}{ }^{2}=.08\right)$. Future research will focus on identifying critical values of feedback characteristics that elicit qualitative shifts in isometric force production strategies.

Dimension: 4 , Delay: 70 , Radius $28 \%$

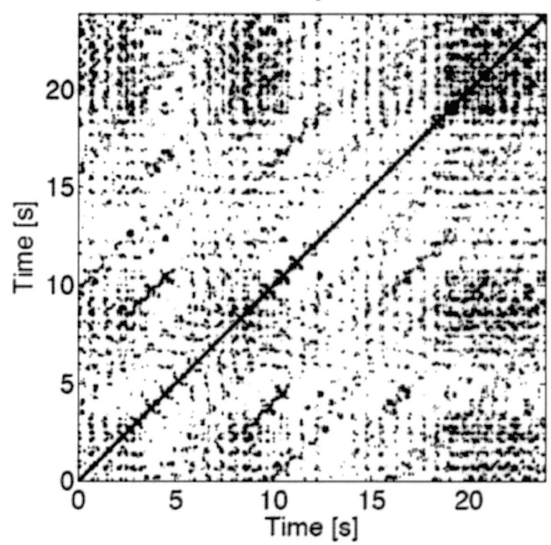

Force produced by participant 5

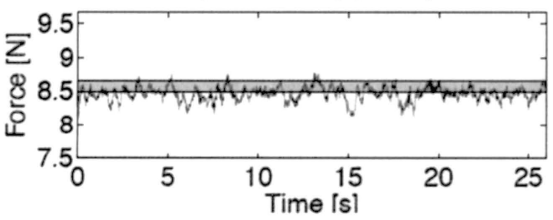

Dimension: 4 , Delay: 70 , Radius $25 \%$

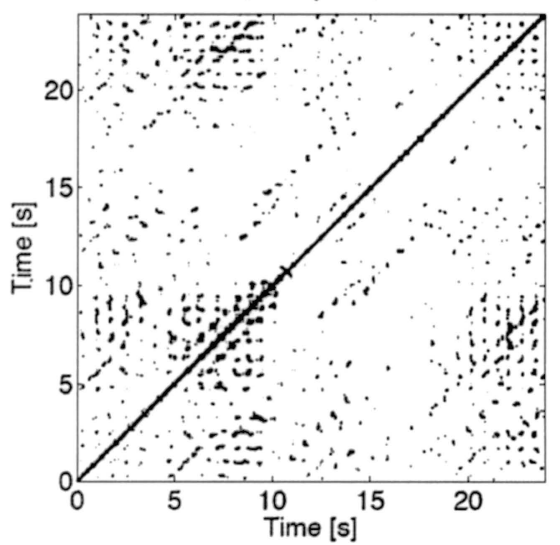

Force produced by participant 5

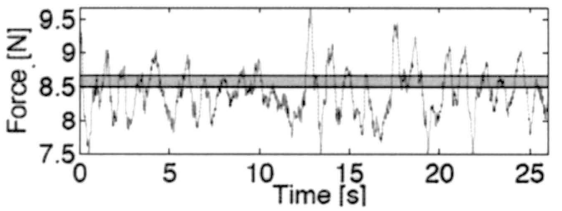

Figure 1. Time series (bottom) of force produced in the $1 \%$ continuous (left panel) and $1 \%$ discrete (right panel) displays and (top) their corresponding recurrence plots. $\% L A M$ is $63 \%$ in the continuous condition and $94 \%$ in the discrete condition. 


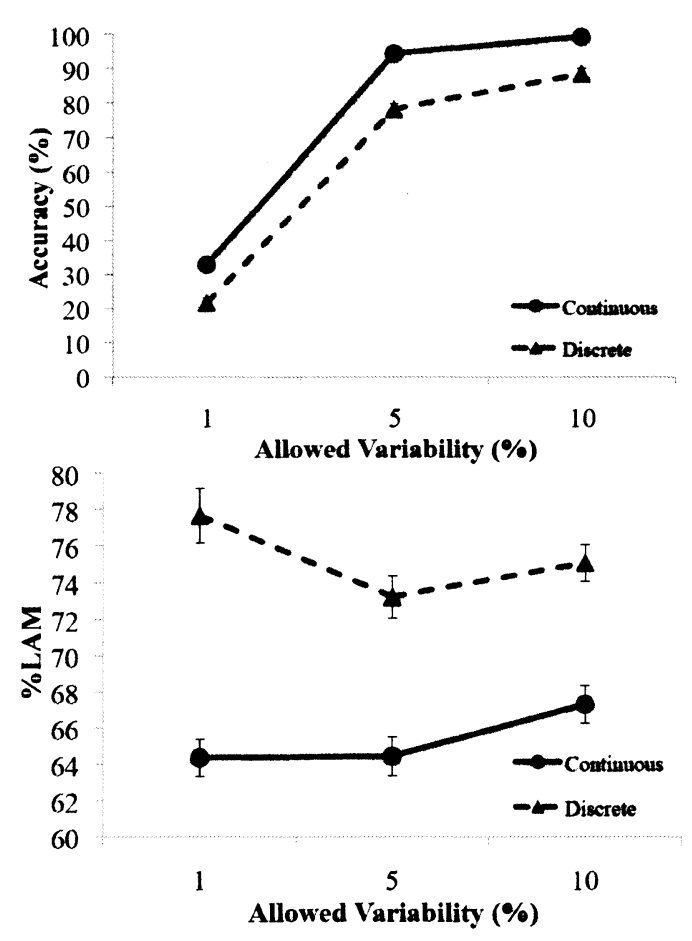

Figure 2. Average values of accuracy (left) and \%LAM (right). Error bars indicate one standard error.

\section{References}

Marwan, N., Wessel, N., Meyerfeldt, U., Schirdewan, A., \& Kurths, J. (2002). Recurrence-plot-based measures of complexity and their application to heart-rate-variability data. Physical Review E, 66, 026702-026702.

Riley, M. A., \& Turvey, M. T. (2002). Variability and determinism in motor behavior. Journal of Motor Behavior, 34, 99-125.

Slifkin, A. B., \& Newell, K. M. (1999). Noise, information transmission, and force variability. Journal Of Experimental Psychology: Human Perception \& Performance, 25, 837-851.

Sosnoff, J. J., Newell, K. M. (2005). Intermittent visual information and the multiple timescales of visual motor control of continuous isometric force production. Perception \& Psychophysics, 67, 335-344.

Webber, C. L., \& Zbilut, J. P. (2005). Recurrence quantification analysis of nonlinear dynamical systems. In M. A. Riley \& G. C. Van Orden (Eds.), Tutorials in contemporary nonlinear methods for the behavioral sciences (pp. 26-95). Retrieved October 27, 2008, from

http://www.nsf.gov/sbe/bcs/pac/nmbs/nmbs.jsp. 
Studies in Perception \& Action $X$

J. B. Wagman \& C. C. Pagano (Eds.)

(C) 2009 Taylor \& Francis Group, LLC

\title{
Microslips in a Manual Placing Task
}

\author{
Ken Yoshida \& Thomas A. Stoffregen
}

University of Minnesota

Microlips are small glitches that occur in manual action. Previous research has documented the existence of microslips in adults and children (e.g., Reed \& Schoenherr, 1992, Suzuki \& Sasaki, 2001). These authors argued that microslips occur during transitions between one action to another. Suzuki \& Sasaki manipulated the complexity of the task situation (e.g., the presence or absence of distracter elements) and found that microslips were more common in more complex task situations.

The task complexity manipulations of Suzuki and Sasaki (2001) were qualitative. We attempted to develop a method in which the frequency of microslips could be related to quantitative manipulation of a control parameter. We examined two preferred modes in manual, pronation and supination. Preferred modes of action have been widely studied including grip configuration (Cesari \& Newell, 1999), intra- and inter-personal grasping (Richardson, et al., 2007), and bimanual finger coordination (Kelso, 1984). We asked participants to grasp a wooden rod and place it upright on a table. To do this, they were obliged to choose between pronate and supinate hand posture in grasping the rod. We varied the initial position of the rod, in terms of its angle relative to the participant. We hypothesized that the occurrence of pronatory or supinatory reaching would be influenced by the angle of the rod, and that at some critical angle there would be a reliable boundary between pronate and supinate reaching. We expected microslips to occur as participants prepared to grasp the rod, and we predicted that microslips would be more common when the rod angle was near the transition between pronate and supinate reaching modes.

\section{Method}

Twenty-one undergraduate students participated. The experimental protocol was reviewed and approval by the institutional review board of the University of Minnesota.

Participants sat in front of the table, with a rod presentation device on either side (Figure 1). The angle of rod was varied, in $15^{\circ}$ increments, from $-15^{\circ}$ to 
$90^{\circ}$. Participants were asked to pull the rod from the rod-base and place it vertically on the table in front of them. There were 80 trials per participant ( 40 for each hand), which were video recorded.

Frequencies of pronation/supination reaching and microslips were coded from the video files. In addition, microslips were categorized using the classification of Reed and Schoenherr (1992). The categories were hesitations, trajectory changes, touches, and hand shape changes.

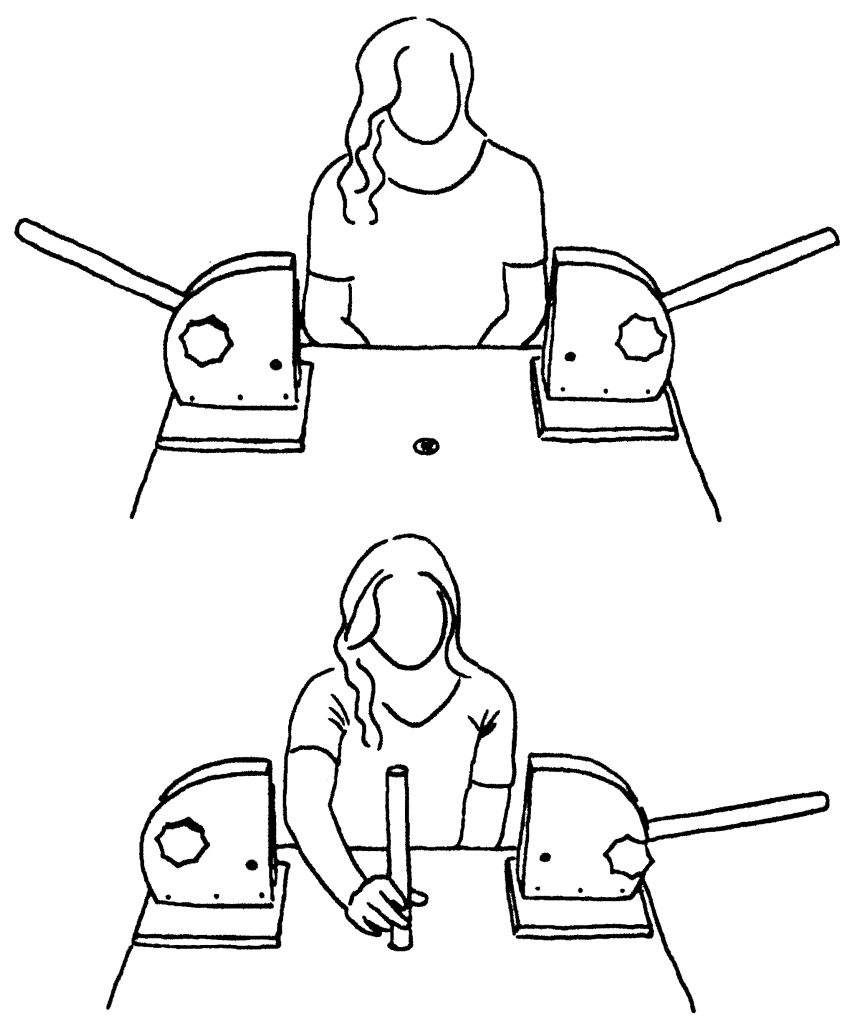

Figure 1. Experimental setting. Two rod-bases were placed on right and left sides of the table. Angles of rod were adjustable with $15^{\circ}$ increments. Participants were instructed to lift and place the rod on the target which was marked on the table.

\section{Results and Discussion}

Contrary to our expectation, rod angle had only a weak effect on pronation/supination. This was because participants elected to use supination in $99.9 \%$ of cases when reaching for the rod. The rare pronate reaches tended to occur when the rod angle was $-15^{\circ}$. Thus, we were not able to test our prediction that microslips would occur near pronation/supination transitions. There 
were a total of seven microslips in the reach/grasp phase. Six of these were touches, and one was a hand shape change.

Our analysis revealed that microslips were more common in the terminal part of the action, when participants were preparing to place the rod on the table. We observed 29 hesitations during rod placement. This result indicates that microslips can occur in relatively simple situations.

Reed and Schoenherr (1992) argued that microslips should tend to occur near transitions of action modes. This insight inspired our attempt to develop a method in which the frequency of microslip occurrence would be related to quantitative manipulation of a control parameter that was related to a transition between two action modes. We developed a grasp-and-place task in which participants were required to choose between pronation and supination of the hand when reaching for the to-be-grasped object. The utility of our method depended upon the occurrence of a transition between pronation and supination. We found some evidence of a transition between reaching modes, but this evidence occurred only at one of the extreme values of our control parameter. In future research, we will attempt to identify values of a control parameter that can reliably lead to transitions between action modes in reaching.

\section{References}

Cesari, P., \& Newell, K. M. (1999). The scaling of human grip configurations. Journal of Experimental Psychology: Human Perception and Performance, 25, 927-935.

Kelso, J. A. S. (1984) Phase transitions and critical behavior in human bimanual coordination. American Jounral o Physiology: Regulatory, Intergrative and Comparative, 15, R1000-R1004.

Reed, E. S., \& Schoenherr, D. (1992). The neuropathology of everyday life: On the nature and significance of micro-slips in everyday activities. Unpublished manuscript.

Richardson, M. J., Marsh, K. L, \& Baron, R. M. (2007). Judging and actualizing intrapersonal and interpersonal affordances. Journal of Experimental Psychology: Human Perception and Performance. 33, 845-859

Suzuki, K., \& Sasaki, M. (2001). The task constraints on selection of potential units of action: An Analysis of microslips observed in everyday tasks. Cognitive Studies, 8, 121-138. 
Studies in Perception \& Action $X$

J. B. Wagman \& C. C. Pagano (Eds.)

(C) 2009 Taylor \& Francis Group, LLC

\section{Chapter 2:}

\section{Interpersonal Coordination}


Studies in Perception \& Action X

J. B. Wagman \& C. C. Pagano (Eds.)

C 2009 Taylor \& Francis Group, LLC 
Studies in Perception \& Action X

J. B. Wagman \& C. C. Pagano (Eds.)

C) 2009 Taylor \& Francis Group, LLC

\title{
Perceiving Affordances for a Dyad
}

\author{
Tehran J. Davis, Michael A. Riley, Kevin Shockley, \\ Kimberly Capehart, \& Justin Fine
}

Department of Psychology, University of Cincinnati

An affordance is an opportunity for action provided by the environment to an organism. Affordances can often be defined as an environmental property scaled by the action capabilities of an organism. For example, an opening affords passing through for an individual whose widest body dimension (usually the shoulder, $S$ ) is less than the dimensions of the aperture $(A)$. However, not all affordances are relative to individuals. It is often the case that people act together to achieve a shared goal. While considerable attention has been given to how affordances constrain individuals, only recently have a few investigations investigated the affordances of groups of individuals engaged in a joint action. For example, Isenhower and colleagues (2005) demonstrated that people are able to distinguish the boundaries between an affordance for oneself and an affordance for a dyad. The present study extended previous findings regarding affordances and joint actions by determining whether individuals were perceptually sensitive to affordances specific to a dyad of which they were a member.

We investigated the affordance of pass-through-ability of an aperture for both individuals and dyads. In individuals, the affordance for passing through an aperture may be expressed as an intrinsic measure, $A / S$ (see Warren \& Whang, 1987 ). As long as the $A / S$ ratio is above some critical value, individuals can pass through a doorway without needing to rotate their shoulders. Warren and Whang found that this critical $A / S>1$, reflecting a margin or "safety buffer" to account for the natural lateral body sway that occurs while walking. Experiment $1 \mathrm{de}-$ termined this critical boundary for dyads.

\section{Experiment 1}

In Experiment 1 we determined the critical $A / S$ value at which both an individual and a dyad could walk through a doorway without rotating their shoulders. 


\section{Method}

Participants $(\mathrm{N}=7$; mean shoulder width $=46.6 \mathrm{~cm})$ were asked to walk through a doorway without touching its perimeter. Starting from $4 \mathrm{~m}$ away, in half of the trials, individuals approached the aperture alone. In the other half, participants performed in a dyad, approaching the aperture alongside a model (shoulder width: $47 \mathrm{~cm}$ ) the entire length of the walk and arriving at the doorway concurrently. Aperture width was varied by trial (by either increasing or reducing width by $5 \mathrm{~cm}$ ). For individuals the apertures ranged from 40 to $90 \mathrm{~cm}$, and for dyads, from $70 \mathrm{~cm}$ to $120 \mathrm{~cm}$.

All trials were recorded by an overhead camera mounted directly above the aperture. Video from each trial was clipped to capture the final $94 \mathrm{~cm}$ of the participants' approach. All video clips were later presented in randomized order to five separate raters. The raters identified trials in which the participant (or in instances in which there was a dyad, either the participant or the model) rotated or adjusted their shoulders in order to fit through the aperture. Inter-rater reliability was determined to be acceptably high $(0.75-0.90)$.

\section{Results and Discussion}

$A / S$ was calculated for each trial (in the dyad condition, $S$ was equal to the combined shoulder widths of the participant and the model). From these data, the critical action boundary between turns and no turns, or the point of subjective equality (where raters indicated a turn $50 \%$ of the time), was calculated by submitting the percent turn scores at each $A / S$ to a probit function. The points of subjective equality were predicted using the best-fitting probit function. A t-test for independent means comparing the estimated points of subjective equality between individual $(\mathrm{M}=1.21)$ and dyad $(\mathrm{M}=1.12)$ conditions was significant, $t(6)=-4.31, p<.01$, such that the critical $A / S$ ratio for dyads was less than individuals walking alone.

The critical $A / S$ for both individuals and dyads indicated the presence of a buffer zone (i.e., $A / S>1$ ) in determining pass-through-ability. However, these results suggest that when individuals were engaged in the task together as a dyad, the resulting joint affordance was not the sum of the two individuals' separate affordances. Had this been the case, we would have identified identical critical $A / S$ ratios across individual and dyad conditions. Instead, critical $A / S$ ratios for individuals were greater than for dyads, indicating that the affordance boundary for dyads is under-additive (less than the sum of two individuals' boundaries).

\section{Experiment 2}

In Experiment 2, we addressed the question of whether or not individuals were perceptually sensitive to the affordance of passing through an aperture for a dyad-specifically, whether observers were sensitive to the under-additivity. 
We also determined whether different types of visual experience were equally adequate to support accurate judgments for oneself, a model, and the dyad.

\section{Method}

Participants $(\mathrm{N}=30)$ ranged in shoulder width from 36.00 to $50.90 \mathrm{~cm}$ (mean $=43.16 \pm 3.75 \mathrm{~cm}$.). Prior to data collection, participants were randomly assigned to one of three conditions: 1) See walk, the participant stood at the end of a hallway $1.8 \mathrm{~m}$ wide and watched a model (from Experiment 1) walk down and back the hallway's length $(10.67 \mathrm{~m})$ five times; 2) Walk with, the participant walked alongside the model maintaining a constant distance as the two walked the length of the hallway 5 times; 3 ) See standing, the participant only viewed the model standing still for one minute prior to the start of the experiment.

Participants were then led into a laboratory to make estimates. The doorway from Experiment 1 was set up inside a chamber $(3 \times 2 \times 2.5 \mathrm{~m})$ enclosed by black curtains hanging from the ceiling. The participants were able look into the chamber via a small view port located at eye-level at the corner of one "wall" of curtains. The doorway was positioned inside the chamber $2.25 \mathrm{~m}$ from the viewport. The participant and model stood outside the chamber with the model positioned $2 \mathrm{~m}$ to the right of the participant, facing perpendicular to the participant at all times.

Participants estimated the minimum aperture width through which they or the dyad could walk (side-by-side in the case of the dyad) without turning the shoulders. Using the method of limits, participants were instructed to inform the experimenter, who was manually moving the doorway, to stop when the doorway reached the perceived critical limit. Two consecutive estimations, corresponding to the two directions of presentation (ascending and descending aperture widths), were averaged together to make a single trial. Type of estimate (self, other, dyad) was presented in counterbalanced blocks of 8 trials for a total of 24 trials. Between trials, participants were instructed to either close their eyes (during the self-judgment condition) or turn to look at the model (during other and for the dyad conditions).

\section{Results and Discussion}

Separate $3 \times 3 \times 8$ ANOVAs were conducted on raw estimates and on estimates scaled to $A / S$. For mean raw estimates, a significant effect was observed for estimate type (self, model, dyad), $F(2,27)=410.65, p<.01, \eta_{p}{ }^{2}=.85$. Bonferroni-corrected post-hoc tests showed that raw estimates in all three estimatetype conditions differed significantly from one another $(p<.0167)$, increasing in value from estimates for self, to estimates for the model, to estimates for the dyad. No significant trial effect was observed, nor for between group comparisons of task experience.

For mean $A / S$, a significant effect for judgment type was also observed, $F(2,27)=32.23, p<.05, \eta_{p}{ }^{2}=.31$. However, post-hoc tests revealed that while mean perceived critical $A / S$ ratios for self (1.14) and model (1.16) did not differ 
from one another, critical $A / S$ for dyads were significantly less than each $(p>$ .05) (see Figure 1).

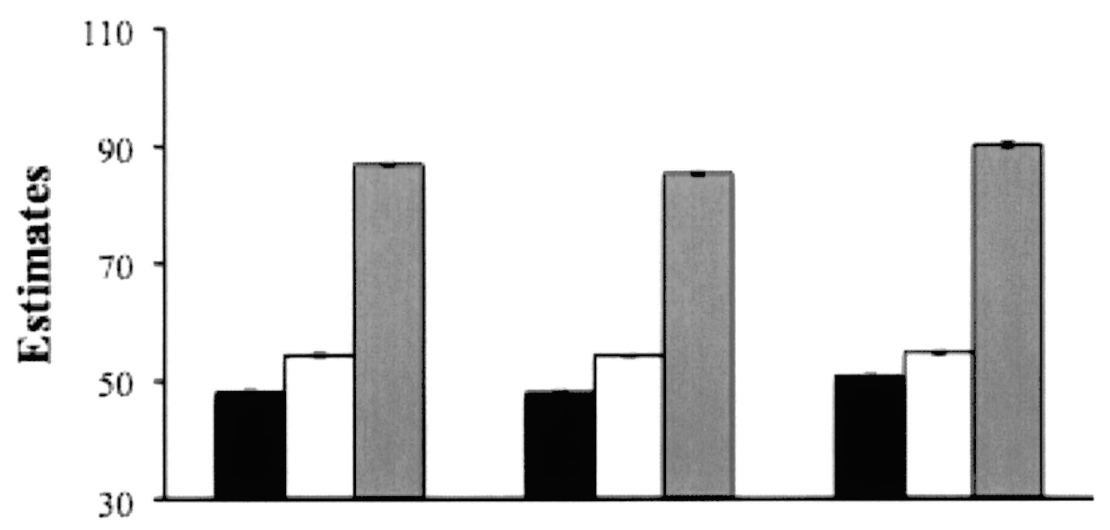

- Self $\square$ Model $\square$ Dyad

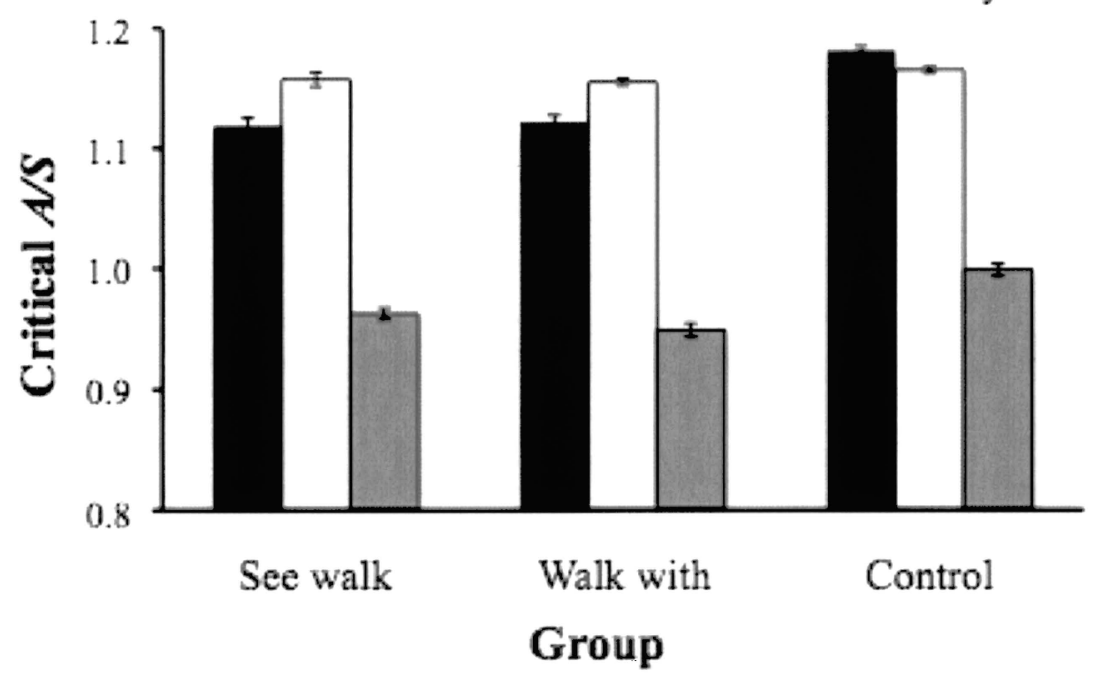

Figure 1. Perceptual reports of Experiment 2 expressed as raw data (top) and $A / S$ ratios (bottom).

Taken together, these results suggest that while individuals were sensitive to absolute differences between themselves and the model, they scaled affordances for both to the same intrinsically scaled $A / S$ values. Furthermore, the critical $A / S$ differences between individuals and dyads mirrors the trend from Experiment 1 , and indicates that participants were sensitive to the affordance of the dyad. 


\section{References}

Isenhower, R. W., Marsh, K. L., Carello, C., Baron, R. M., \& Richardson, M. J. (2005). The specificity of intrapersonal and interpersonal affordance boundaries: Intrinsic versus extrinsic metrics. In H. Heft \& K. L. Marsh (Eds.), Studies in perception and action, viii. Mahwah, NJ: Erlbaum.

Warren, W. H., \& Whang, S. (1987). Visual guidance of walking through apertures: Body-scaled information for affordances. Journal of Experimental Psychology: Human Perception and Performance, 13, 371383. 
Studies in Perception \& Action X

J. B. Wagman \& C. C. Pagano (Eds.)

(C) 2009 Taylor \& Francis Group, LLC

\title{
Inter- and Intra-personal Coordination in Autistic and Typically-Developing Children
}

\author{
Robert W. Isenhower ${ }^{1}$, Kerry L. Marsh ${ }^{1}$, Paula Silva ${ }^{1}$, \\ Michael J. Richardson ${ }^{2}$, and R. C. Schmidt ${ }^{3}$ \\ ${ }^{1}$ Center for the Ecological Study of Perception and Action, \\ University of Connecticut \\ ${ }^{2}$ Department of Psychology, Colby College \\ ${ }^{3}$ Department of Psychology, College of the Holy Cross
}

Autism Spectrum Disorder (ASD) is a pervasive developmental disorder that is currently diagnosed on the basis of a triad of behavioral indicators: (1) deficits in social interaction, (2) deficits in language for communication, and (3) restricted and repetitive interests and activities (DSM-IV-TR, 2000). Points (1) and (2) are fundamentally coordinative in nature. Therefore, studying coordination in children with ASD may reveal subtleties of the dynamics of intrapersonal and interpersonal coordination not apparent in typically-developing (TD) children.

Coordinated behavior has been shown to be constrained by a coupled oscillator dynamic and can be described by the so-called HKB equation (Haken, Kelso, \& Bunz, 1985):

$$
\dot{\phi}=\Delta \omega-a \sin \phi-2 b \sin 2 \phi+\sqrt{Q} \zeta
$$

Predictions of the HKB equation have found substantial support in a pendulum-swinging paradigm. This is so whether the pendulums are held by a single individual (Kugler \& Turvey, 1987) or by. two individuals (Schmidt \& Turvey, 1994). Moreover, interpersonal coordination is apparent even without explicit instruction to coordinate (Richardson, Marsh, \& Schmidt, 2005). Critical to the current study, the HKB equation is not limited to the wrist-pendulum paradigm. The current study uses an interpersonal rocking chair paradigm (cf. Richardson, Marsh, Isenhower, Goodman, \& Schmidt, 2007) and an intrapersonal drumming task (cf. Brakke, Fragaszy, Simpson, Hoy, \& Cummins-Sebree, 2007) to assess coordination in ASD and TD children. 


\section{Method}

Children (ranging from 2.5 to 8 years of age) participated in two tasks: interpersonal rocking and intrapersonal drumming. Out of the total sample of children $(\mathrm{N}=23)$, TD children $\left(\mathrm{N}_{\mathrm{TD}}=7\right)$ and children with ASD $\left(\mathrm{N}_{\mathrm{ASD}}=7\right)$ were age-matched based on a battery of standard clinical assessments.

During the interpersonal task child/parent pairs were seated in rocking chairs (Fig. 1, left). Movement data were collected from sensors attached to the chairs. The parent's rocking chair was weighted so that its natural frequency closely matched the natural frequency of the child's rocking chair $(\approx 0.83 \mathrm{~Hz})$. A metronome that only the parent could hear was used in order to keep the rocking frequency of the parent constant. This also ensured that any spontaneous coordination observed was due to the child entraining to the parent. Parents read a story to their child in order to keep their attention. Children were never told to rock; however, most children did rock spontaneously.
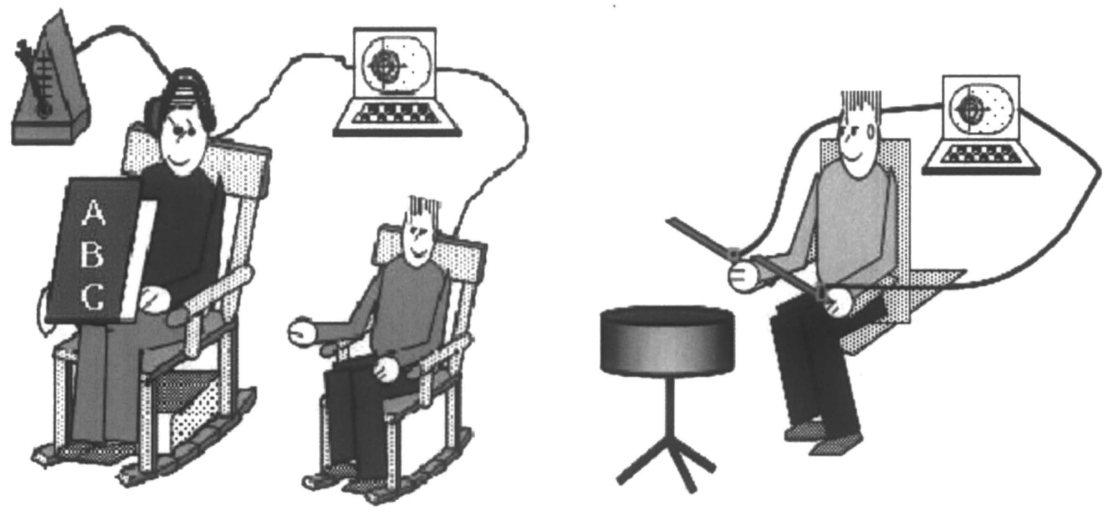

Figure 1. (left) Interpersonal rocking and (right) intrapersonal drumming.

During the intrapersonal task, children were first shown how to hold the drumsticks and how to strike the drum in an in-phase and an anti-phase manner. They were then instructed to strike the drum continuously for two 45 second trials (one in-phase and one anti-phase) at their own preferred pace. The order of the trials was counterbalanced across participants. Movement data were collected from sensors attached to the drumsticks (Fig. 1, right).

\section{Results and Discussion}

For interpersonal rocking, continuous relative phase (CRP) was examined. The amount of time that the child's chair was in a particular phase relation with the parent, in nine steps from $0^{\circ}$ (in-phase) to $180^{\circ}$ (anti-phase), was assessed for the age-matched sub-sample $(\mathrm{N}=14)$ of the children. A 2 (Group: TD vs. ASD) by 9 (Phase bins) mixed ANOVA revealed a significant Group $\times$ Phase bin interaction, $F(8,96)=2.57, p<.05, \eta_{\mathrm{p}}^{2}=.18$. The CRP results for the interper- 
sonal rocking task indicate the pattern of coordination observed for TD children is different than the pattern observed for children with ASD. TD children exhibit more in-phase coordination than children with ASD; the latter showed no discernable preferred phase region (Fig. 2, left).
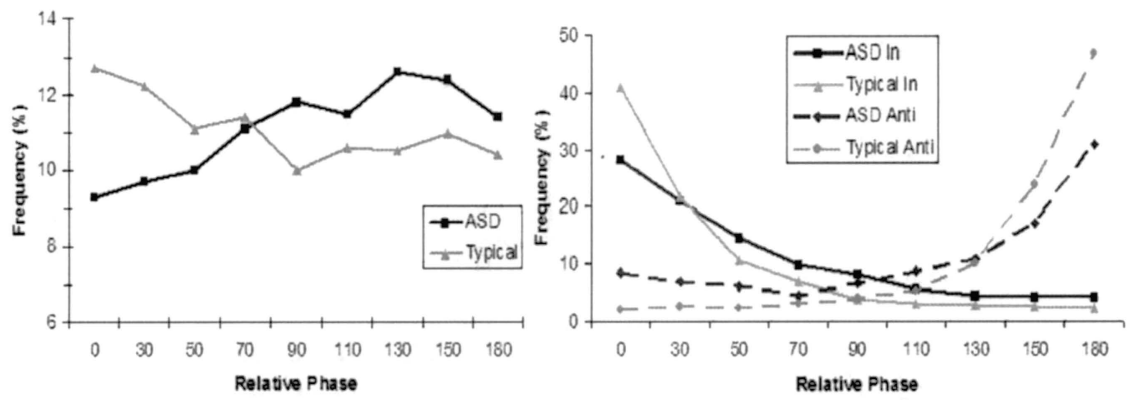

Figure 2. (left) Amount of time spent by each group in each of the phase bins. (right) Amount of time spent in the required phase (in- or anti-phase) by the two groups of children.

For intrapersonal drumming, CRP was examined separately for in-phase coordination and for anti-phase coordination using parallel Group $\times$ Phase region mixed ANOVAs for the age-matched sub-sample $(\mathrm{N}=14)$. The analyses revealed a significant Group $\times$ Phase bin interaction for both. For in-phase coordination, $F(8,96)=3.81, p<.001, \eta_{\mathrm{p}}^{2}=.20$, an independent samples t-test found a significant difference between the in-phase bin of the two groups, $t(12)$ $=2.28, p<.05$. For anti-phase coordination, $F(8,96)=4.23, p<.001, \eta_{\mathrm{p}}^{2}=$ .21 , an independent samples t-test found a significant difference between the anti-phase bin of the two groups, $t(12)=2.59, p<.05$. Overall, the CRP analyses of the drumming data revealed significantly more in-phase and anti-phase coordination for TD children than for children with ASD. Typical children are better able to maintain the required phase during drumming (Fig. 2, right).

Cross recurrence quantification (CRQ) analysis was also applied to the rocking and drumming data. CRQ measures the dynamic similarity between two time series embedded in a reconstructed phase space. No significant effects were found for rocking. For drumming, however, differences were apparent. For antiphase coordination, TD children had significantly greater $\%$ recurrence than ASD children, $t(12)=2.29, p<.05$, significantly longer meanline, $t(12)=3.14$, $p<.05$, and a marginally longer maxline, $t(12)=1.82, p<.08$. For in-phase coordination, in contrast, TD children had a marginally shorter meanline than ASD children, $t(12)=2.12, p<.06$. Previous research has shown that for CRQ analysis maxline and meanline are indices of attractor strength and \% recurrence is an index of noise (Richardson, Schmidt, \& Kay, 2007). Under this analysis, it is possible that typically developing children assemble oscillatory drumming movements that have less noise than children with ASD. However, given that the meanline difference between TD children and children with ASD is the op- 
posite for in-phase coordination and anti-phase coordination, the results concerning attractor strength of the drumming movements are equivocal.

Overall, the results of the current study find coordination differences between children with ASD and TD children for both interpersonal and intrapersonal tasks. Uninstructed interpersonal coordination between parent and child in the rocking task revealed that TD children spent more time in phase with the parent whereas ASD children showed no particular preferred phase relation. For the intrapersonal drumming task, which required maintaining a particular phase relation between their own hands, both groups of children were successful: $0^{\circ}$ was produced under the in-phase instruction, $180^{\circ}$ was produced under the antiphase instruction. However, TD children were better at maintaining the required phase relation. The results of CRQ analysis indicate that TD children assemble oscillatory movements with less noise than children with ASD, although it is unclear if the attractor strength of the oscillatory movements in either group differs from each other.

\section{References}

American Psychiatric Association. (2000). Diagnostic and statistical manual of mental disorders: DSM-IV-TR. Washington, DC: American Psychiatric Press, Inc.

Brakke, K., Fragaszy, D. M., Simpson, K., Hoy, E., \& Cummins-Sebree, S. (2007). The production of bimanual percussion in 12- to 24-month old children. Infant Behavior \& Development, 30, 2-15.

Haken, H., Kelso, J. A. S., \& Bunz, H. (1985). A theoretical model of phase transitions in human hand movements. Biological Cybernetics, 51, 347-356.

Kugler, P. N., \& Turvey, M. T. (1987). Information, natural law, and the selfassembly of rhythmic movements. Hillsdale, NJ: Lawrence Erlbaum Associates.

Richardson, M. J., Marsh, K. L., Isenhower, R. W., Goodman, J., \& Schmidt, R. C. (2007). Rocking together: Dynamics of intentional and unintentional interpersonal coordination. Human Movement Science, 26, 867-891.

Richardson, M. J., Marsh, K. L., \& Schmidt, R. C. (2005). Effects of visual and verbal information on unintentional interpersonal coordination. Journal of Experimental Psychology: Human Perception and Performance, 31, 62-79.

Richardson, M. J., Schmidt, R. C., \& Kay, B. A. (2007). Distinguishing the noise and attractor strength of coordinated limb movements using recurrence analysis. Biological Cybernetics, 96, 59-78.

Schmidt, R. C., \& Turvey, M. T. (1994). Phase-entrainment dynamics of visually coupled rhythmic movements. Biological Cybernetics, 70, 369-376.

Acknowledgements. This research was supported by a Cure Autism Now (CAN) Grant and a National Science Foundation Grant \# 0240277. 
Studies in Perception \& Action X

J. B. Wagman \& C. C. Pagano (Eds.)

(C) 2009 Taylor \& Francis Group, LLC

\title{
The Influence of Interpersonal Interaction on Postural Coordination Dynamics in a Suprapostural Task
}

\author{
Manuel Varlet, Ludovic Marin, Julien Lagarde, \& Benoît G. Bardy \\ Efficiency and Deficiency Laboratory, University Montpellier1, Montpellier, France
}

Previous postural coordination studies have shown that multi-segment postural coordination can be described by the relative phase between the ankle and hip in a suprapostural task (i.e., when posture is investigated within a goaldirected task; Bardy, Marin, Stoffregen, \& Bootsma, 1999). Research revealed that when participants "posturaly" track the sinusoidal motion of a target, two stable patterns emerge from the ankle-hip relative phase: an in-phase mode (close to $20^{\circ}$ ) for low target frequency displacements and an anti-phase mode $\left(180^{\circ}\right)$ for high target frequency motions. Increasing target frequency produced an abrupt change from in-phase to anti-phase coordination and inversely, decreasing frequency produced a change from anti-phase to in-phase coordination (Bardy, Oullier, Bootsma, \& Stoffregen, 2002).

For a decade, studies have been investigating postural coordination when participants were alone and almost none have examined posture in interpersonal situations. In our everyday life, however, posture it is not an end in itself, but rather it is often used to (involuntarily) communicate, work with someone, practice a sport and so on. Shockley, Santana and Fowler (2003) have shown objective evidence of postural influence in interpersonal situation. However, this study only measured a global assessment of postural activity and did not investigate the postural coordination of paired participants. The goal of the current study, therefore, was to investigate whether postural coordination can be influenced during a visual interpersonal interaction. Based on previous research on postural coordination (Bardy et al., 1999; 2002) we know that in order to perform a suprapostural task, participants modify their postural coordination. They adapt the ankle-hip relative phase to the situation they are involved in. Consequently, we hypothesized that in interpersonal situations participants would adapt their own ankle-hip relative phase to be coordinated with their co-actor. 


\section{Method}

Twenty participants stood in front of a target simulated on a monitor and were instructed to track the A-P oscillations of the target with an increase and a decrease of frequency ranging from $0.10 \mathrm{~Hz}$ to $0.75 \mathrm{~Hz}$ with step of $0.05 \mathrm{~Hz}$ (see Bardy et al. 2002 for more details). No instructions were given on the postural coordination they could adopt. They performed the tracking task in two conditions (Figure 1): paired (Duo condition) and alone (Solo condition).
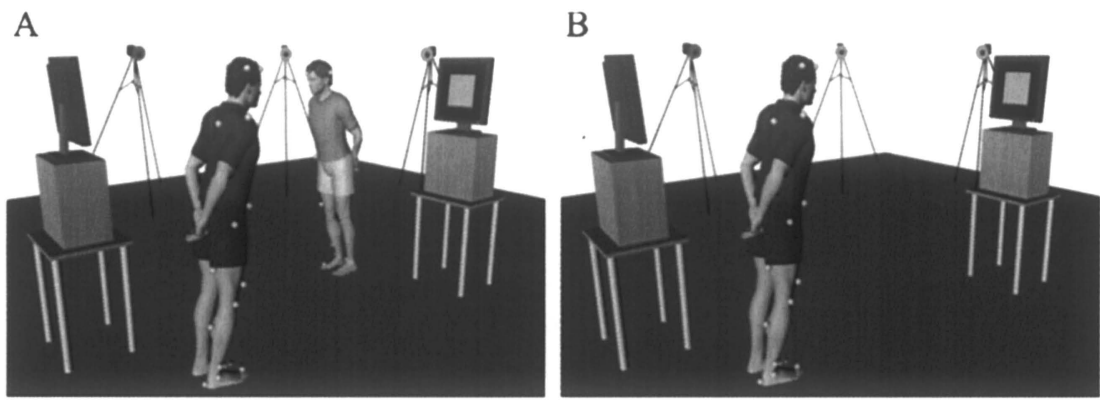

Figure 1. In the Duo condition (A) participants saw their co-actor in their peripheral view. In the Solo condition (B) participants were alone to perform the tracking task.

To analyze the influence of the interpersonal interaction on the spontaneous postural coordination dynamic, three variables were computed: 1) distribution and 2) standard deviation of the ankle-hip relative phase of each participant, indicating the coordination modes produced and their stability; 3 ) absolute difference between the postural transition frequencies (TF) of each paired participant in order to determine the influence of interpersonal interaction on the transition frequency.

\section{Results and Discussion}

1) The distribution of ankle-hip relative phase angles across nine phase regions was submitted to a 2 (Condition) $\times 9$ (Phase Region) repeated measures ANOVA. Using the Greenhouse-Geisser correction, this analysis yielded a significant main effect of phase region $(F(8,152)=63.26, p<.05)$ and no significant interaction between phase region and condition $(F(8,152)=0.20, p>.05)$. These results showed that 1) anti-phase (and in-phase) coordination mostly emerged from this task and 2) there were no postural coordination differences in Duo and Solo conditions (Figure 2). Globally, participants did not produce more in-phase or anti-phase pattern during interpersonal interaction. 


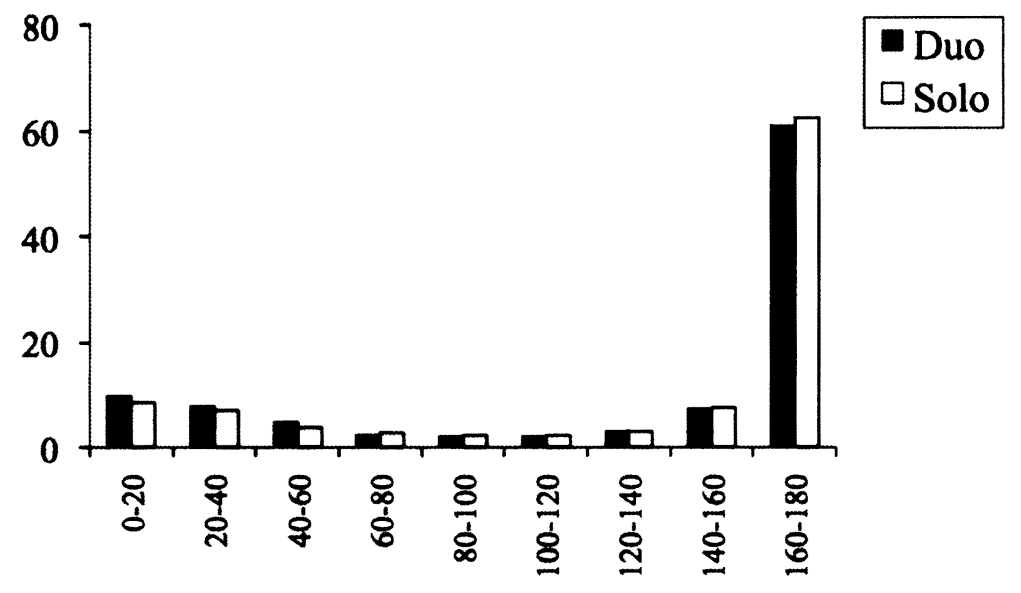

\section{Ankle-Hip Relative Phase Region ( ${ }^{\circ}$ )}

Figure 2. Distribution of ankle-hip relative phase angles for Duo (black) and Solo (white) conditions.

2) The standard deviation of ankle-hip relative phase was $8.24(\mathrm{SD}=4.36)$ in Duo condition and $8.56(\mathrm{SD}=5.44)$ in Solo condition. No difference in postural coordination stability was observed between Duo and Solo conditions. Interpersonal interaction did not affect the stability of postural coordination patterns produced by participants. This result suggests that the strength of the coupling of postural coordination is strong enough to not be influenced by the presence of the other participant. This is consistent with previous interpersonal coordination studies and indicates that intrapersonal coordination is more stable than visual interpersonal coordination (e.g., Schmidt et al., 1998).

3) The mean of the absolute difference between the transition frequencies (TF) was $0.19 \mathrm{~Hz}(\mathrm{SD}=0.14)$ for Duo condition and $0.24 \mathrm{~Hz}(\mathrm{SD}=0.14)$ for Solo condition. One factor ANOVA with repeated measures showed significant difference between these two conditions $(\mathrm{F}(1,8)=10.78 p<.05)$ (Figure 3 Top), revealing that when paired, participants spontaneously modified the frequency of their postural transition from one coordination mode to the other. Globally this result shows that during visual interaction, participants shifted postural coordination patterns either earlier or later than when alone, in order to adopt a similar postural pattern as the one performed by their co-actor (Figure 3 Bottom).

In conclusion, this study reveals that the exchange of visual information between two people can directly influence their postural coordination dynamic. This study can also be considered as a new paradigm in investigating postural coordination in a more social (and ecological) situation. 

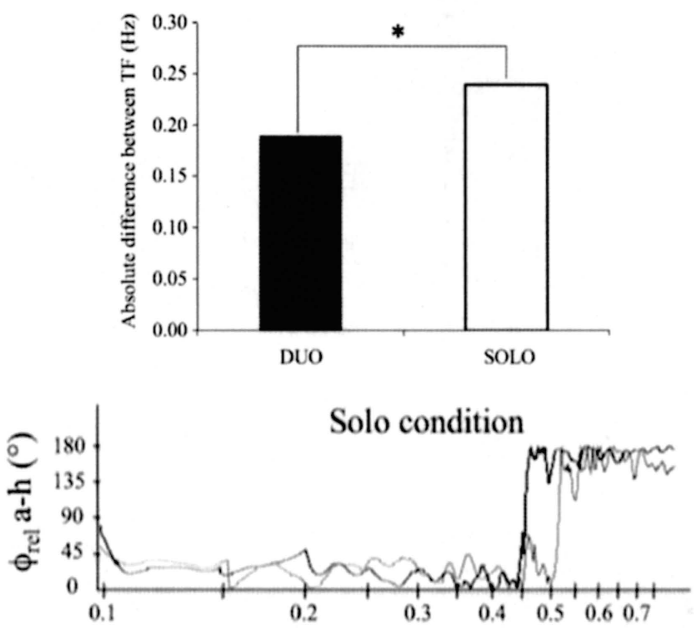

- Participant 1

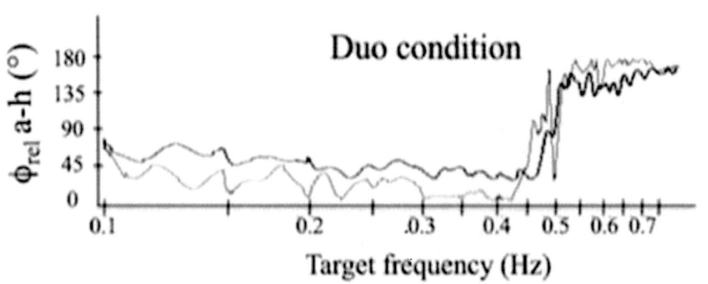

- Participant 2

Figure 3. (Top) Absolute difference between TF in Duo and Solo conditions. (Bottom) Ankle-hip relative phase of two participants in Duo and Solo conditions illustrating that the presence of someone influenced participant's postural transition.

\section{References}

Bardy, B.G., Marin, L., Stoffregen, T.A., \& Bootsma, R.J. (1999). Postural coordination modes considered as emergent phenomena. Journal of Experimental Psychology: Human Perception and Performance, 25, 1284-1301.

Bardy, B.G., Oullier, O., Bootsma, R.J., \& Stoffregen, T.A. (2002). Dynamics of human postural transitions. Journal of Experimental Psychology: Human Perception and Performance, 28, 499-514.

Schmidt, R.C., Bienvenu, M., Fitzpatrick, P.A., \& Amazeen, P.G. (1998). A comparison of intra- and interpersonal coordination: Coordination breakdowns and coupling strength. Journal of Experimental Psychology: Human Perception and Performance, 24, 884-900.

Shockley, K., Santana, M.V., \& Fowler, C.A. (2003). Mutual interpersonal postural constraints are involved in cooperative conversation. Journal of Experimental Psychology: Human Perception and Performance, 29, 326332.

Acknowledgements. This research was supported by SKILLS, an Integrated Project (IST contract \#035005) of the Commission of the European Community. 
Studies in Perception \& Action X

J. B. Wagman \& C. C. Pagano (Eds.)

(C) 2009 Taylor \& Francis Group, LLC

\title{
Gaze Coordination between Car Drivers and Passengers: An Observation
}

\author{
Hiroshi Inou ${ }^{1}$, Mamoru Sawada ${ }^{1} \&$ Hiroyuki Mishima ${ }^{2}$ \\ ${ }^{1}$ DENSO Corporation, Japan, ${ }^{2}$ Waseda University, Japan
}

Passengers riding in a vehicle operated by an unskilled driver (e.g., a newly licensed driver) may feel fear or anxiety. One possible reason for such feelings is the rough operation and consequent rough movement of the vehicle. However, there seems to be an underlying perceptual cause as well. Consider the following two empirical facts: (1) an unskilled driver tends to focus their gaze on the near side of his/her front view while driving and (2) a passenger (riding in a vehicle with an unskilled driver), may be unconsciously induced to gaze at the near side of his/her front view relative to a distant landscape, although this is not the case when a skilled driver operates the vehicle. Why might this kind of gaze entrainment between a driver and a passenger occur? We propose that the structure of global optic flow presented to a passenger (or the passenger's visual system) is lawfully affected by a driver's "perceptual" skill (or the driver's gaze control, which could be considered as a reflection of his/her perceptual exploration) because the driver's operations that lawfully constrain the optic flow experienced in the vehicle must be controlled by the driver's perception. Therefore, when a driver's skill is poor, a passenger's gaze movement might be unstable, and he/she would feel uneasy. As the first step to addressing this gaze entrainment problem, we conducted an in-vehicle experiment.

\section{Method}

The experiment was conducted at a test track in the DENSO Abashiri Test Center (Hokkaido, Japan). The participants were: one skilled driver, who had been employed as a test driver at DENSO Corporation for 15 years, and one ordinary driver. In the "skilled driver condition," the skilled driver operated the test vehicle and the ordinary driver rode along in the passenger seat; in the "ordinary driver condition," the positions were reversed. The participants' gaze directions relative to the car's orientation were measured by recording their eye movements at $20 \mathrm{~Hz}$ with an eye tracking system (NAC Image Technology, EMR-8B). The movement data were smoothed using a Kalman filter. Two suc- 
cessive curves, "Corner A" (right-hand curve; radius = $100 \mathrm{~m}$ ) and "Corner B" (left-hand curve; radius $=120 \mathrm{~m}$ ), were defined as the experimental trials. Both the participants were asked to approach the first corner with an approximate velocity of $60 \mathrm{~km} / \mathrm{h}$ while driving and look ahead when riding as the passenger.
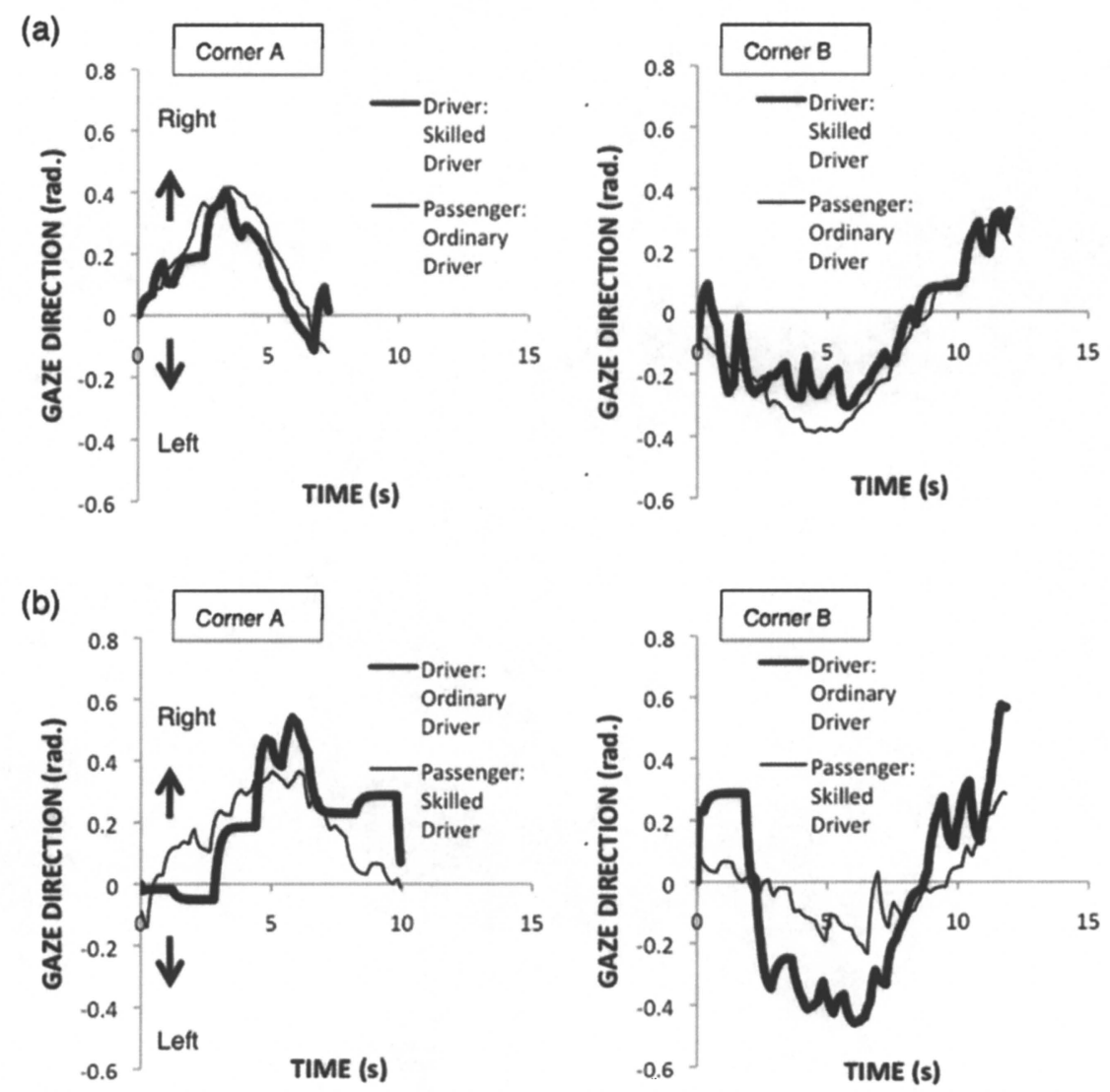

Figure 1. Gaze direction relative to the car's orientation as a function of time: (a) skilled driver condition; (b) unskilled driver condition

\section{Results and Discussion}

The participants' gaze directions relative to the car's orientation in the skilled driver and ordinary driver conditions are shown in Figure 1. This figure illustrates the tendency for coordination between a driver's and passenger's gaze directions. Moreover, this coordination tendency appears to be stronger in the skilled driver condition than in the ordinary driver condition.

To examine the degree of gaze coordination between the driver and passenger, we used cross-recurrence quantification analysis (CRQA; Marwan, N., \& 
Kurths, 2002; Marwan, 2003; Shockley, 2005; Zbilut \& Webber, 1992). In this analysis, the following CRQA parameter settings were used: embedding dimension $=6$, delay $=0.25 \mathrm{~s}$, radius $=0.15$ radian, norm $=$ Euclidean. The data used in the CRQA were not normalized.

(a)
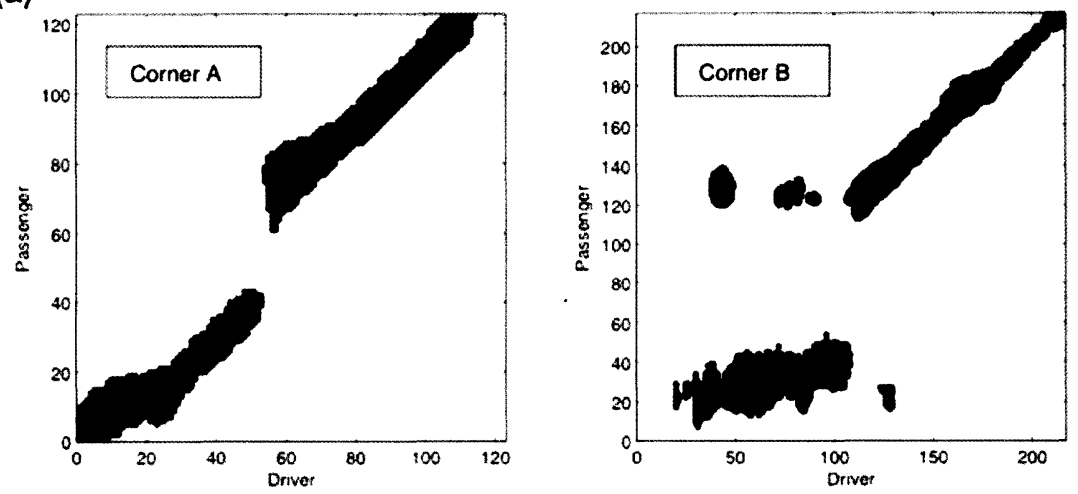

(b)
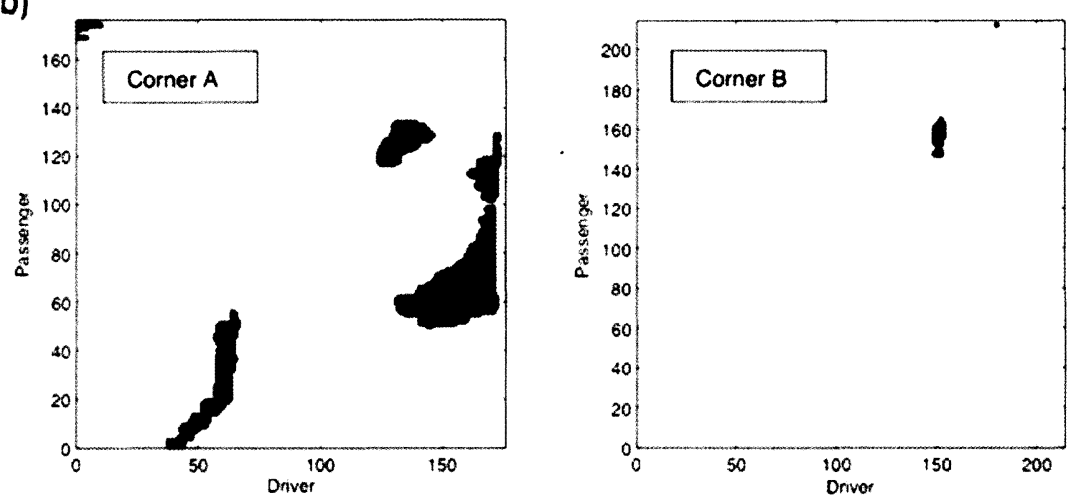

Figure 2. CRPs of the gaze directions of the driver and passenger in the ordinary driver condition: (a) skilled driver condition; (b) unskilled driver condition

The cross-recurrence plots (CRPs) for each data pair in each condition are shown in Figure 2. The \%recurrence in each CRP is as follows: 0.104 and 0.08 for Corners A and B, respectively, in the skilled driver condition; 0.05 and 0.002 for Corners A and B, respectively, in the ordinary driver condition. These results support the strong coordination tendency in the skilled driver condition and the weak coordination tendency in the ordinary driver condition.

A driver's skill is directly reflected by the vehicle's behavior. Frequent changes in acceleration caused by poor operation of the vehicle by an unskilled driver produce disturbances in not only the driver's and passenger's bodies but also the optic flow field in front of the vehicle. On the other hand, the smooth and dexterous operations by a skilled driver will prevent such vibrations, main- 
tain a dynamically stable optic flow, and guide the passenger's as well as the driver's gaze to an adequate point in the field of view. In future studies, we plan to explore the specific optic flow information that guides drivers' and passengers' gaze movements.

\section{References}

Marwan, N. (2003). Encounters with neighbors: Current developments of concepts based on recurrence plots and their applications. Doctoral thesis, University of Potsdam, Potsdam, Germany.

Marwan, N., \& Kurths, J. (2002). Nonlinear analysis of bivariate data with cross recurrence plots. Physics Letters A, 302(5-6), 299-307.

Shockley, K. (2005). Cross recurrence quantification of interpersonal postural activity. In M. A. Riley \& G. C. Van Orden (Eds.), Tutorials in contemporary nonlinear methods for the behavioral sciences (pp. 142-177).

Retrieved October 13, 2006, from http://www.nsf.gov/sbe/bcs/pac/nmbs/nmbs.jsp

Zbilut, J. P., \& Webber, C. L., Jr. (1992). Embeddings and delays as derived from quantification of recurrence plots. Physics Letters A, 171, 199-203. 
Studies in Perception \& Action X

J. B. Wagman \& C. C. Pagano (Eds.)

(C) 2009 Taylor \& Francis Group, LLC

\title{
Target Distance Influences Interpersonal Postural Coordination
}

\author{
Alison Smith ${ }^{1}$, Christie Pelzer ${ }^{1}$, Russ Giveans ${ }^{1}$, Kevin Shockley ${ }^{2}$, \\ $\&$ Thomas A. Stoffregen ${ }^{1}$ \\ ${ }^{1}$ University of Minnesota, ${ }^{2}$ University of Cincinnati
}

\begin{abstract}
When pairs of people converse while standing, their body sway exhibits spontaneous coordination (Shockley et al., 2003). When each member of a dyad converses with a different person, sway within the dyad is not coordinated, indicating that the effect is related to the interpersonal interaction. The strength of interpersonal coupling can be influenced by variations in the conversational interaction (Shockley et al., 2007). In addition, coordination might be influenced by characteristics of the targets of the conversation. For example, when dyads converse about something that they can see, postural coordination might be influenced by adjustments to posture to facilitate looking at objects of mutual interest.

Stoffregen et al. (1999) found that the magnitude of spontaneous body sway (in individuals) was related to the distance of visual targets: Sway was reduced when people fixated nearby targets, relative to sway during fixation of distant targets. This effect was functional in the sense that a given magnitude of sway would tend to have a more disruptive effect on fixation of nearby targets.

We evaluated whether distance-related effects of vision on stance in individuals might also affect the coordination of posture between members of a conversing dyad. To test this possibility, we replicated the task partner manipulation in the facing away condition from Shockley, et al. (2003), and added a manipulation of the distance of visual targets. We predicted that target distance would influence the sway of individuals in each dyad, and that target distance would influence the strength of interpersonal postural coordination.
\end{abstract}

\section{Method}

Thirty students at the University of Minnesota participated in return for course credit. Participants were organized into 15 conversational pairs.

We used the picture puzzle task employed by Shockley et al. (2003), using the same cartoon pictures as that study. Within pairs, pictures were generally 
similar in appearance to one another with 10 subtle differences. In the Near condition, the cartoon pictures were $14.0 \times 10.2 \mathrm{~cm}$ and were placed $0.5 \mathrm{~m}$ from the participants. In the Far condition, the same pictures were enlarged to $69.9 \times$ $47.0 \mathrm{~cm}$ and were placed $2.5 \mathrm{~m}$ from the participants to achieve the same visual angle as the Near condition. Members of each dyad stood facing away from each other, on opposite sides of the emitter of a magnetic tracking system (Polhemus). Sensors were placed on the head (using an elastic headband) and hip (using tape) of each participant, and were sampled at $30 \mathrm{~Hz}$. There were four trials per condition yielding 16 trials per participant pair. Participant pairs were asked to discuss their respective pictures to identify the 10 differences between the pictures. Participants either discussed their pictures with the other participant (Together) or with an experimental confederate (Confederate).

We used a $2 \times 2$ design, with Target Distance (Far vs. Near) and Conversational Partner (Together vs. Confederate), and four trials (per dyad) in each condition. Each trial was $120 \mathrm{~s}$ in duration. The two time series for a given pair for a given body location (head vs. hip) were submitted to a cross-recurrence analysis (see Shockley et al., 2003 for a more detailed discussion of this analysis). Mean \%REC (the \% of body configurations shared by a participant pair) and MAXLINE (the length of the longest consecutive sequence of recurring points for a given pair) for a given condition for a given body location for each participant pair were submitted to a $2 \times 2$ analysis of variance (ANOVA). Individual participants' positional data for head and hip body locations were also submitted to a $2 \times 2$ ANOVA.

\section{Results and Discussion}

Cross-recurrence analysis. There were no significant effects in the MAXLINE variable. For the \%RECUR variable the main effect of Partner was not significant for the head or the hips. Thus, we failed to replicate the effect of conversational partner on interpersonal postural coordination (Shockley et al., 2003, 2007), though as illustrated in Figure 1 the data showed the same trend as Shockley et al. (2003). However, for head motion the main effect of target distance on \%RECUR was significant, $F(1,14)=5.35, p<.05$. As predicted, variations in the visual task (target distance) influenced the strength of interpersonal postural coupling. Participants showed less shared postural activity at the head for far targets as compared to near targets. The data for head motion are summarized in Figure 1. 


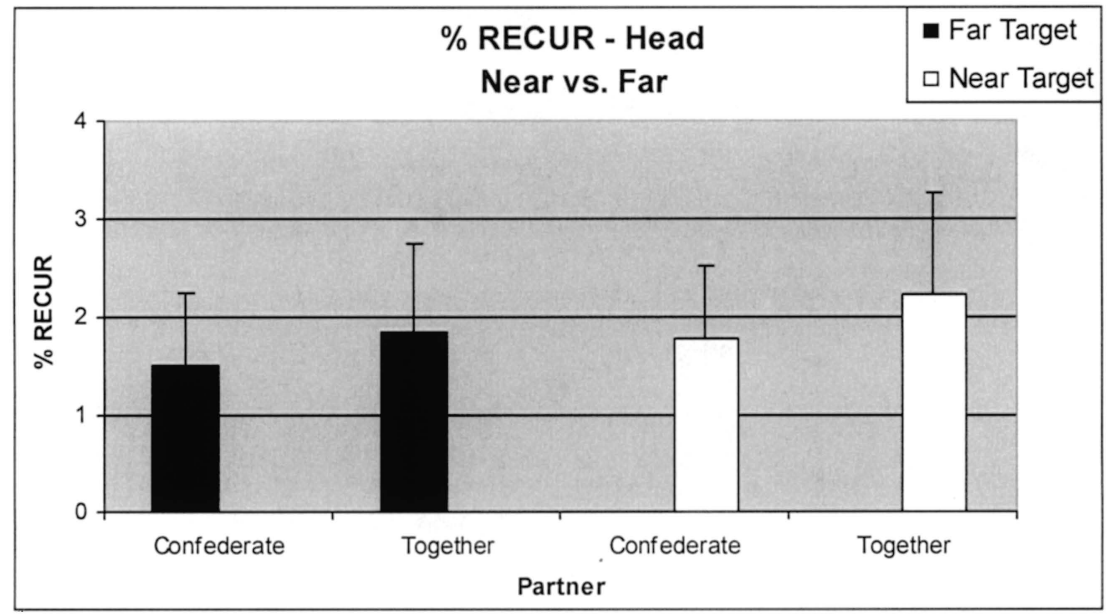

Figure 1. Results of cross-recurrence analysis, showing \%RECUR for dyadic head movements as a function of conditions.

Positional variability. For the head, the mean variability of position in the ML axis was $1.30 \mathrm{~cm}$ in the Near condition, and $1.58 \mathrm{~cm}$ in the Far condition, $F(1,29)=14.737, p<.01$. For the hips, the ML means were $0.75 \mathrm{~cm}$ and 0.91 $\mathrm{cm}$, respectively, $F(1,29)=11.173, p<.01$. These effects replicate Stoffregen et al. (1999), and confirm our prediction. However, in the AP axis the influence of target distance on head movement was in the opposite direction $\left(\mathrm{Mean}_{\mathrm{Near}}=2.02\right.$ $\mathrm{cm}$, Mean $\left._{\mathrm{Far}}=1.77 \mathrm{~cm}\right), F(1,29)=8.46, p<.01$.

We also found a significant effect of conversational partner on the positional variability of the head in the AP axis $\left(M_{\text {ean }}{ }_{\text {Together }}=1.76 \mathrm{~cm}\right.$; Mean ${ }_{\text {Confederate }}=$ $2.03 \mathrm{~cm}), F(1,29)=5.47, p<.01$. A similar effect was observed by Stoffregen, Giveans, Villard, Yank, \& Shockley (2009), and indicates that interpersonal influences on postural control are not limited to the dependent variables that emerge from cross-recurrence analysis.

Shockley et al. (2003) did not find a visual influence of the other participant on postural coordination. By contrast, in the present study we did find a visual influence of the target distance. Taken together, the results of our analysis of positional variability suggest that participants controlled AP body movement with respect to each other, and simultaneously controlled ML body movement with respect to the visual targets. Comparison of our two types of analysis (cross-recurrence and positional variability) suggest that the distance of visual targets influenced both the sway of individuals and the coupling of sway within dyads. One explanation for this pattern of results is that participants may have exhibited greater amplitude of eye movement in the Near condition as compared to the Far condition resulting in greater postural sway in the A-P direction (cf. Stoffregen, Bardy, Bonnet, \& Pagulayan, 2006). This could be evaluated by tracking the eye movement patterns of participants in future studies. 


\section{References}

Shockley, K., Baker, A. A., Richardson, M. J., \& Fowler, C. A. (2007). Articulatory constraints on interpersonal postural coordination. Journal of Experimental Psychology: Human Perception and Performance, 33, 201-208.

Shockley, K., Santana, M. V., \& Fowler, C. A. (2003). Mutual interpersonal postural constraints are involved in cooperative conversation. Journal of Experimental Psychology: Human Perception and Performance, 29, $326-$ 332.

Stoffregen, T. A., Bardy, B. G., Bonnet, C. T., \& Pagulayan, R. J. (2006). Postural stabilization of visually guided eye movements. Ecological Psychology, 18, 191-222.

Stoffregen, T. A., Giveans, M. R., Villard, S., Yank, J., Shockley, K. (2009). Interpersonal postural coordination on rigid and non-rigid surfaces. Ms. under review.

Stoffregen, T. A., Smart, L. J., Bardy, B. G., \& Pagulayan, R. J. (1999). Postural stabilization of looking. Journal of Experimental Psychology: Human Perception \& Performance, 25, 1641-1658. 



\section{References}

\section{Chapter 1: Action 8 Coordination}

Beek, P. 1., Peper, C. E., \& Stegeman, D. F. (1995). Dynamical models of movement coordination. Human Movement Sciences, $14,573-608$.

Blau, 1. J. C., Stephen, D. G., Carello, C., \& Turvey, M. T. (under review). Prism adaptation of underhand throuing: Rotational inertia and the primary and latent aftereffects.

Brooks, R. L., Nicholson, R. 1., \& Fawcett, A. J. (2007). Prisms throw light on developmental disorders.

Neurophysiologica, 45, 1921-1930.

Fernandez-Ruiz, 1., \& Diaz, R. (2006). Prism adaptation and aftereffect: Specifying the properties of a procedural memory system. Learning \& Memory, 7, 193-198.

Fernandez-Ruiz, J., Hall-Haro, C., Diaz, R., Mischner, J., Vergara, P., \& LopezGarcia, J. C. (2000) Learning motor synergies makes use of information on muscular load. Learning \& Memory, 7, 193-198.

Frank, T. D., Blau, 1. 1. C., \& Turvey, M. T. (2009). Ninlinear attractor dynamics in the fundamental and extended prism adaptation paradigm. Physics Letters $A$, $373(11), 1022-1030$.

Kelso,1. A. S. (1995) Dynamic patterns. MIT Press: Cambridge, $M A$.

Schoner, G. S. \& Kelso, J. A. S. (1988). A synergetic theory of environmentallyspecif Ied and learned patterns of movement coordination: I. Relative phase dynamics. Biological Cybernetics, 58, 71-80. Acknowledgments. This research was supported by National science Foun

dation grant SBR $04-23036$.

(6) 2009 Taylor \& Francis Group, LLC

Functional Tuning of Action to Task Constraints in Tool-Use: The Case of Stone Knapping Blandine Bril, Robert Rein, \& Tetsushi Nonaka Ecole des Hautes Etudes en Sciences Sociales, France Tool use appears as a privileged entry to understand goal directed actions, 
the organism being not simply directed toward the goal, but rather directed by

the goal (Shau, 1987). The aim of the current study is to characterize the exper

tise in the earliest known instance of human tool-use, stone knapping, from the

perspective where the emphasis is on the goal while the movement is viewed as

driven by task constraints. In stone knapping, one stone (hammer) is used to strike another (core) to

remove a sharp-edged piece ( $f$ lake) according to the fracture mechanism called

conchoidal fracture. Counter intuitively, in" conchoidal fracture, it has been dem

onstrated that the force of the blow does not playa major role in determining the

dimension of the detached flake (Dibble \& Pelcin, 1995). It does, however, play

a critical role in determining whether or not a flake can be produced in the first

place. Therefore, kinetic energy of the hammer at impact is one of the essential

task constraints for flake production, and we hypothesized that if actions are

controlled in a functionally specific manner, knappers would maintain the $\mathrm{ki}$

netic energy of the hammer at impact invariant irrespective of the mass of ham

mers used. In other words, the achievement of the goal, the removal of a flake, is

driven in such a way to meet the constraints of the task, that is, to produce the

required kinetic energy of the hammer at impact of the strike. Method Nine participants with different knapping 
experience (two experts, three in

termediates and four novices) participated in the

experiment. Two hammers with

different mass (heavy: 400g, light: 250g) were used. Flint weighing between $2-3$

$\mathrm{kg}$ was used as cores. Striking movements were recorded using an Ascension

miniBIRDC magnetic marker system set. at a recording

frequency of $100 \mathrm{~Hz}$.

One sensor was attached to the backside of the hand. Each participant produced

twenty flakes with each hammer. The trajectory length of the hammer was cal

culated based on the trajectory of the hand sensor in 30 space from the maxi

mum vertical height to the point of impa<; t. The kinetic energy was calculated

using the formula $\mathrm{E}$ kin $={ }^{\infty} \mathrm{m} v 2$ ( $\mathrm{v}=$ resultant velocity). A linear mixed-model

analysis incorporating hammer weight and skill level in the fixed-effects struc

ture and subject as a random effect was used for statistical analysis, and Bon

ferroni-corrected post-hoc tests were used for multiple comparisons. Results and Discussion 590 strikes contributed to the analysis. Success rate of flaking per group

was $84 \%$ for experts, $55 \%$ for intermediates, and $49 \%$ for novices. Hammer trajectory length. Statistical analysis indicated a significant main

effect for hammer weight, $F(!, 577)=79.1, p<.001$, as well as a significant in

teraction between hammer weight and skill level, $F(1,577)$ $=3.8, p<.05$. Par 
ticipants used longer trajectories when striking with the lighter hammer. velocity at impact. Statistical analysis for striking velocity indicated a sig

nificant main effect for hammer weight, $F(1,577)=52.4, P$ $<.001$. Kinetic energy. Statistical analysis indicated significant differences between

hammer conditions, $F(1,577)=14.3, p<.001$. Post-hoc test further revealed

that there were no significant differences in kinetic energy between hammer

conditions for experts, while the differences for intermediates and novices were

both significant $(p<.01)$. All remaining effects were nonsignificant. The results of the experiment showed that modem stone knappers adjust

their movements according to task constraints. When using a lighter hammer

participants across all groups increased the velocity of the hammer to achieve

perceived necessary kinetic energies. However, only expert knappers were able

to adjust their movement kinematics in a way which left the resulting kinetic

energy unaltered between hammer conditions. In contrast, novice and intermedi

ate skilled knappers exhibited different kinetic energies between the two condi

tions due to disproportional increases in striking velocity for the lighter hammer.

The results indicate that knapping skill is characterized by functional tuning to

task constraints. In addition, a general trend was observed where the strikes of

experts tend to involve lower kinetic energy compared to novice and intermedi 
ate knappers. The fact that the success rate of detachment of flakes by strikes

was highest for experts and lowest for novices implies that kinetic energy for

novices was more than what was needed to detach a flake, while experts con

trolled their actions just sufficient to achieve the goal. The results stress the in

fluence of perceptual parameters for the control of actions. For successful ac

tion, the actor must establish the functional relationship between himself, the

tool, and the environment. Overall, the results of this study highlight the importance of detecting the

constraints of the task, and regulating actions accordingly. The characteristics of

expert skills we found, fits nicely to the definition of dexterity given by Bern

stein, who wrote "Dexterity is the ability to create a perfect key for any emerg

ing lock (Bernstein, 1996, p. 215).

Bernstein, N. A. (1996). Dexterity and Its Development. Mahuah, No: Laurence Erlbaum Associates.

Dibble, H. L., \& Pekin, A. (1995). The Effect of Hammer Mass and Velocity on Flake Mass. J. Archaeol. Sci, $22,429-439$.

Shaw, R. E. (1987). Behavior with a purpose [Review of the book Goaldirected Behavior]. Contemporary Psychology, $32,243-245$. Acknouledgements. This research was funded by the European Union pro

ject HANDTOMOUTH.

(6) 2009 Taylor \& Francis Group, LLC Variability of Uni-Manual Pendulum 0scillation at and away from Resonance Dobromir G. Dotov 1 , Damian G. Stephen 1 , Till D. 
Frankl, \& M. T. Turveyl, 2

lCenter for the Ecological Study of Perception and Action, University of Connecticut 2Haskins Laboratories Extensive research based on the dual-pendulum paradigm (Kugler \& Tur

vey, 1987) has helped to establish overarching dynamical laws of intraand in

ter-personal coordination (Amazeen, Amazeen, \& Turvey, 1998). Can the study

of uni-manual pendulum ascillation too 'be informative about dynamic con

straints on motor behavior and coordination? In order to address this question

our experiment focused on the coordination between the rhythmic motor beha

vior of suinging a hand-held pendulum and an auditory metronome as an envi

ronmental stimulus. Previous research with the same design (using phase space

reconstruction to characterize the dynamics) found that the system could be de

scribed as having feuer active degrees of freedom, less noise, and a greater pre

diction horizon when running at its resonance as compared to when running at

louer or higher rates (Goodman, Riley, Mitra, \& Turvey, 2000). In this way, the

"comfort" that the central nervous system experienced in a resonant configura

tion could be defined in terms of minimization of controlled variables and max

imization of predictability of chaotic dynamics. In order to extend the understanding of such a system, we delved further in

to the issue of variability. A vast array of research 
bility of motor behavior is not just noise extrinsic to the movement determinism

but actually plays an essential role in helping the system establish its functional

ly appropriate behavior (for a review see, Riley \& Turvey, 2002). In this respect,

Abarbanel suggests that "the range of alt"matives

available to which one can

control a system is larger if chaotic motion, seen as a wide ranging exploration

of state space, is allowed" (1996, p. vi). We can sharpen our initial question by

using Abarbanel's insight as a guiding intuition. What can the properties of the

oscillation variability tell us about the system? Does pushing it away from re

sonance mean pushing it auay from its stable state, thus, inducing a potential for

the system to search for alternative stable states? Is the amount and structure of

variability going to express this potential? 9 To begin answering these questions, we used drift-diffusion analysis based

on generalized Langevin equation seeking to reconstruct the stochastic dynamics

of frequency entrainment. This approach has been recommended in light of its

potential ability to emphasize the intrinsic role played by the stochastic compo

nents of a dynamical system (Van Mourik, .Daffertshofer, \& Beek, 2006). On another level of analysis we focused on Detrended Fluctuation Analysis

(DF A) as a means to quantify the fractal properties of 
wrist (Peng, Havlin, Stanley, \& Goldberger, 1995). The relation between aspects

of the changing structure of variation, as expressed by pouer-law coefficients,

and a system's moving closer or farther away from equilibrium in a statistical

mechanical sense has been discussed in the literature. For example, a direct rela

tion has been demonstrated between the fractal properties of the distribution

function of a dissipative system and both its nonequilibrium steady state and

relaxation touards equilibrium (Gilbert, Dorfman, \& Gaspard, 2000). Important

ly, such phenomena should not be considered as isolated to physics or physical

chemistry. Entropy has also been used as a predictor of the creation of new

structure in the face of acquiring a novel cognitive skill (Stephen, Dixon, \&

Isenhower, in press). Method Seven right-handed

participants performed a uni-manual pendulum

suinging task maintaining a steady phase relative to an auditory metronome.

They received six trials in each of the three conditions of slow $(.67 \mathrm{~Hz})$, reson

ance $(.83 \mathrm{~Hz})$, and fast metronome $(1.33 \mathrm{~Hz})$ in a random sequence. The reso

nant frequency of the wrist-pendulum system was determined following the pro

cedures outlined in Kugler and Turvey (1987) to calculate the period of the sim 
ple equivalent pendulum. A goniometer provided measures of wrist deviations

(radial and ulnar). Absolute angular displacement furnished the same number of

data points per condition for DF A while a peak-extracting algorithm derived

trial series of rescaled cycle frequencies serving as the domain of the drift

diffusion analysis. Results and Discussion $A$ repeated-measures ANOVA on the coefficient Q quantifying the stochas

tic term in the drift-diffusion analysis of frequency entrainment found a signifi

cant effect of rate, $F(2,12)=5.77, p<.05$. A significant quadratic trend, $F(!, 6)$

$=8.39, p<.05$ (Figure $1 A)$, indicates that the noise term is minimal at resonance

and increases as pendulum frequencies deviate from resonance. Similar analysis

yields a significant effect for the DFA H coefficient, $F(2,12)=34.23, p<.001$.

As Figure $1 \mathrm{~B}$ shows, $\mathrm{H}$ for the fast pace was different from both resonance $(\mathrm{p}<$

$.01)$ and slow $(p<.01)$ but they did not differ from each

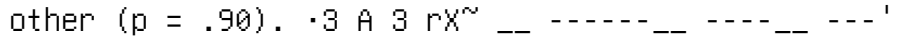

Slow Resonance Fast 0scillation Rate J: : C 0.8 Q) $\cdot \mathrm{u}$ ;;:: ] $0.6 \cup C l c \sim 0.4 \cup(/)$ c : : $0.2 \sim$ B slow Resonance Fast 0scillation Rate Figure 1. Means and standard error bars for the (A) frequency entrainment noise

coefficient $Q$ and (B) fluctuation $H$ exponent. At first blush, these results support our initial hypothesis that variability at

non-resonance rates should be special in informative ways. At the level of fre

quency entrainment, which also can be defined as the amount of the behavioral 
cycle's deviation from target frequency, noise was augmented by deviation from

resonance. A possible interpretation is that maintaining synchrony with the sti

mulus became harder at these rates. With regard to the noise at the level of urist

motions, the higher $H$ coefficient for fast trials, a signature of more complex

fluctuations, can be interpreted in accord with the literature already discussed as

indicative of a pendulum swinger being moved farther away from her equili

brium rate. Under such an interpretation, our study is congruent with the hypo

thesis that one route for a nonlinear system with many degrees of freedom to

dissipate excessive stress is to augment its fluctuation complexity. Dur results, however, introduce some ambiguity. Setting aside the quadratic

trend in the frequency entrainment noise term, there is no difference between

resonance and fast paces $(p>.25)$. The pattern is reversed for mean $\mathrm{H}$ exponents

--there is no difference between slow and resonance. Why did two different

patterns characterize two different levels of analysis? A tentative explanation relies on the conception that state space below and

above resonance might allow for different solutions. Drawing the stimulus

human-pendulum system away from resonance induces breaking of symmetry.

In the low-rate case the broken symmetry is with respect to cycle duration whe 
reas in the high-rate case the broken symmetry is with respect to increasing

Abarbanel, H. D. I. (1996). Analysis of chaotic data. New York, NY: Springerverlag.

Amazeen, P. G., Amazeen, E. L., \& Turvey, M. T. (1998). Dynamics of human intersegmental coordination: Theory and research. In D. Rosenbaum \& c. Collier (Eds.), Timing of behavior: Neural, computational, and psychological perspectives. Boston, MA: MIT Press.

Gilbert, T., Dorfman, R., \& Gaspard, P. (2000). Entropy production, fractals, and relaxation to equilibrium. Physical Review Letters, 85(8), 1606-1609.

Goodman, L., Riley, M. A, Mitra, S., \& Turvey, M. T. (2000). Advantages of rhythmic movements at resonance: minimal active degrees of freedom, minimal noise, and maximal predictability. Journal of Motor Behavior, 32(1), 3-8.

Kugler, P. N., \& Turvey, M. T. (1987). Information, natural law, and the selfassembly ofrhythmic movements. Hillside, NJ: Erlbaum.

Peng, C.-K., Havlin, S., Stanley, H. E., \& Goldberger, A L. (1995). Quantification of scaling exponentials and crossover phenomena in nonstationary heartbeat time series. Chaos, 5(1), 82-87.

Riley, M. A., \& Turvey, M. T. (2002). Variability of determinism in motor behavior. Journal of Motor Behavior, $34(2), 99-125$.

Stephen, D. G., Dixon, J. A., \& Isenhower, R. W. (in press). Dynamics ofrepresentational change: Entropy, action, and cognition. Journal of Experimental Psychology: Human Perception and Performance.

van Mourik, AM., Daffertshofer, A, \& Beek, P. J. (2006). Deterministic and stochastic features of rhythmic human movement. Biological cybernetics, 94(3), 233-244. Acknowledgments. Preparation of this manuscript was supported by a grant

from the University of Connecticut Research Foundation.

(6) 2009 Taylor \& Francis Group, LLC 
by Persons with Doun Syndrome and Those with other Forms of Mental Retardation Shogo Hirata 1 , Hideguki okuzumi 1, Mitsuru Kokubun 1 \& Masaguki Kumai 2 iTokyo Gakugei University 2Tohoku University This study compares the performance of a tray-carrying task by persons

with Down syndrome and those with other forms of mental retardation, and cla

rifies the features of motor skills execution in persons with Down syndrome.

The tray-carrying task is a motor task devised by kokubun (1999). This task

requires subjects to carry a tray bearing a glass filled with water as fast as possi

ble over a distance of 3 meters, but without spilling the water. The time taken

and amount of water spilled are measured. Kokubun (1999) compared perfor

mance of this task by children with Down syndrome and by children with other

forms of mental retardation. The results shoued that children with Down syn

drome were indeed slower than children with other types of mental retardation,

but the amount of water spilled was no different between the two groups. That is

to say, while persons with Down syndrome were slow, they were not inaccurate.

Although the above findings of Kokubun (1999) have a great impact in changing

the general view that persons with Down syndrome were 'clumsy', feu studies

have been conducted from this perspective. In this study, ue conducted the same 
motor task to confirm the findings of kokubun (1999), and we conducted a

normal walking task to compare to the performance of the tray-carrying task. Method Thirteen persons with Doun

syndrome (7 males, 6 females) aged 14 to 48

years (29 \pm 11.8 years) and 13 persons with other forms of mental retardation $(6$

males, 7 females) aged 13 to 48 years (29 \pm 11 . 0 years)

without sensory and

motor impairments were used as subjects. In this study, subjects with Doun

syndrome were diagnosed by medical doctors, and no one with Autism was in

cluded in the group with other forms of mental retardation.

For the tray-carrying task, subjects were given a tray with a glass (9 cm

height, $7 \mathrm{~cm}$ diameter at the top, $4.5 \mathrm{~cm}$ at the bottom, and a capacity of $225 \mathrm{ml}$ )

filled with of $200 \mathrm{mI}$ water at the start line and instructed to carry it to a goal line

$3 \mathrm{~m}$ away as fast as they could without spilling it. The time to reach the goal line

was measured with a stop watch. The amount of water remaining in the glass on

completion of the task was measured with a measuring cylinder, and this value

was subtracted from $200(\mathrm{ml})$ to calculate the amount of water spilled. The aver

age of two trials was used as the representative value for that subject. In normal

walking, subjects were requested to walk normally for $3 \mathrm{~m}$ to compare to the

performance of the tray-carrying task. The time was 
measured. In addition, the

adjustment ratio (the time of the tray-carrying task divided by that of normal

walking) was also calculated. Results and Discussion Table 1 summarizes the mean (M) and standard deviation (SO) of each of

the measures for both groups. Table 1 . Tray-carrying performance by group Tray-carrying task Time, sec Amount of Normal Adjustment water spilled, ml walking, sec ratio M SO M SO M SO M SO

Down $7.98 \quad 4.04 \quad 0.08 \quad 0.28 \quad 4.26 \quad 1.21 \quad 1.97 \quad 1.14$ syndrome

Mental $6.54 \quad 2.83 \quad 1.08 \quad 1.55 \quad 4.60 \quad 2.27 \quad 1.52 \quad 0.43$

retardation Persons with Doun syndrome took more time to complete the task than

those with other forms of mental retardation. However, the time required for the

normal walking task did not differ between the groups. Persons with Down syn

drome spilled less water than the others. Although the adjustment ratio of per

sons with Down syndrome was larger than that of the others, a 2 (Subject: Down

Syndrome vs. Mental Retardation) $\times 2$ (Task condition: tray-carrying vs. nor

mal walking) analysis of variance (ANOY A) yielded no significant differences

between the groups ( $F$ 1, 24=0.40, p>.05), but did yield significant differences

between the task conditions ( F 1,24=21.97, $p<.05$ ). The interaction was not signif

icant ( $F$ 1,24=2.16, p>.05). In the amount of water spilled and adjustment ratio, a

one-way (subject group) ANOYA yielded significant differences only in the 
spillage (water: $F$ 1,24=5.23, p<.0s. ratio: $F$ 1,24=1.61, p>.0S). In normal walking, persons with Down syndrome perform at the same

speed as those with other forms of mental retardation. But, in the tray-carrying

task, persons with Doun syndrome tend to take more time than for their normal

walking, and behave more cautiously and accurately than people with other

forms of retardation. These results accord with the findings of kokubun (1999).

In recent years, Latash (2008) suggested that persons with Doun syndrome do

not have major qualitative deficits in their motor control mechanisms, and that

their clumsy movement was a product of an adaptive strategy to compensate for

their delayed decision-making or cerebellar dysfunction. His vieus are consis

tent with the results in our study. Bernstein (1996)

acutely pointed out 'a de

mand for dexterity is not in the movements themselves but in the surrounding

conditions.' In further investigation, we would like to examine the ecological

reality of 'tray-carrying' for persons with Down syndrome and those with other

forms of mental retardation using various task conditions (i.e., the size of the

glass or amount of water were changed). In our short study, we cannot consider

the effect of the subject's intelligence level on task performance. We will ad 
Bernstein, N. A. (1966). On Dexterity and Its Development. Mahwah, N.T: Laurence Er lbaum Associates.

Kokubun, M. (1999). Are children with Down syndrome less careful in performling a tray-carrying task than children with other types of mental retardation? Perceptual and Motor Skills, 88, 1173-1176.

Latash, M. L. (2008). Neurophysiological Basis of Movement (Second Edition). Champain, IL: Human Kinetics.

Acknowledgements. The authors would like to thank the participants who

made this work possible.

J. B. Wagman \& C. C. Pagano (Eds.)

(6) 2009 Taylor \& Francis Group, LLC observation of Action Slips in a Young Child Nagya Hirose Kyoto Notre Dame University This study investigated a child's action slips during acquisition of a new

daily skill. Action slips are the errors that occur when people do an action that

is not intended. This study differs in two respects from previous studies on ac

tion slips (e.g., Norman, 1981; Reason, ${ }^{9} 984$ ). One difference is that we ob

served a young child instead of adults. Most studies on action slips have been

conducted with adults, and little attention has been devoted to children's slips up

to now. Developmental research is needed to elucidate the nature of action

slips. To the best of our knowledge, this is the fIrst study to report the child's

action slips in a daily setting. The other difference from previous studies is that whereas most studies on

action slips have employed the diary method, this study did not. There are sev

eral difficulties in the diary method. For example, Reason 
(1984) argued that, in

diary studies, there are at least three kinds of biases

(volunteer bias, selection

bias, and recording bias). In addition, it'is almost

impossible for children to

keep diaries for their action slips. Thereby, we used video recordings instead of

diaries. It is often pointed out that diary studies

underestimate the frequency of

slips. People often miss writing down slight slips in their diary, whereas video

recoding method can record all of them. Thus, the method can obtain a more

accurate count of the frequency of slips. Additionally, it can eliminate the selec

tion and recording biases. In this study, we coded microslips as well as action slips. Microslips are

subtle miscues that occur in manipulation tasks (Reed, Palmer, \& schoenherr,

2009). We identified action slips and microslips according to Hirose's (2007)

coding scheme. We first defined the basic units of action and described se

quences of action by using these units. Then we identified slips as deviations

from the normal sequence, and discriminated between action slips and micro lips.

Action slips are deviations across multiple basic units, whereas microslips are

within one basic unit. Consequently, our definition of slips is rather wider than

the previous studies on action slips. To explore the developmental nature of action slips, we observed a child's 
mouth rinsing activity during toothbrushing. We chose this behavior because

we found frequent action slips in the preliininary observations. In addition, the

recording was easy to accomplish because the activity occurred in the same

place at around the same time. Method the only participant of this study was a 4 -year-old girl. Her mouth rinsing

activities during toothbrushing after dinner were observed for 4 months (from

4:0 to 4:4). Her daily routine of toothbrushing was as follows: First, she brushed

her teeth by herself. Second, her parent thoroughly brushed them again. Fi

nally, she rinsed her mouth by herself. She was able to rinse her mouth by her

self a few weeks before the time when the study began. In order to record her mouth rinsing activities, a network camera (AxIS

207W) was installed at the side of a washstand in her house. Video recordings

were done automatically by using the mot.ion detection function built in the re

cording system. All video files were saved to a PC connected to the network.

Video files including the participant's mouth rinsing activities were selected to

be analyzed. The recording system was always in operation through the period

of study, but the target activities were occasionally not recorded due to malfunc

tion of the system or the participant's absence from home. Thus, we obtained 95 
day's video files for analysis. Results and Discussion In the first analysis, we derived a "modal route" (Joe,

Ferraro, \& Schuartz,

2002) of the mouth rinsing activity without any slips. The route was as follous:

ascend step; take cup; hold cup under tap; tum on tap; tum off tap; sip water;

suish water around in mouth; spit water; empty cup; put down cup; descend

step; wipe mouth with towel. We identified any deviations from this route as

slips. Then, we divided these slips into action slips and micros lips according to

the aforementioned criteria. A series of slips was counted as one action slip or

microslip, and those slips that included both action slips and microslips were

coded as one action slip. The analysis yielded a total of 62 slips. Next, we classified slips into five types according to the previous studies of

action slips. However, some microslips that could not be categorized into these

types were labeled as others. Results of this

classification are shoun in Table 1.

Captures were the most frequent type, accounting for $45.2 \%$ of all slips. Capture. Another activity may capture control of the intended activity. The

participant often veered from the hand washing activity. Redoing. Repeated actions that need no repetition in the normal situation.

The participant turned on the tap twice. Anticipation.

skip a necessary action and perform the next step. The par

ticipant turned on the tap before holding the cup under it. Sequential. Sequential errors other than anticipations. The participant at 
tempted to pick up the cup in an inappropriate situation. Hesitation. Common microslips including small pauses during movement.

The participant suddenly stopped reaching for the cup, and then resumed reach

ing. 17 Finally, we examined developmental changes in slips by dividing the period

of study into halves (Figure 1). As shoun in Figure 1, action slips decreased

drastically in the second half. This suggests that the participant rapidly acquired

the skill of mouth rinsing. In contrast, microslips decreased slightly, suggesting

that micros lips may continue through the time period. In concluding, we should note that diary studies substantially underestimate

the frequency of action slips, estimating one per day at most (Jonsdottir

Adolfsdottir, Cortez, Gunnarsdottir, \& Gustafsdottir, 2007). It seems surprising

that, in this study, we found a number of slips during a relatively brief activity.

Diary method reveals cognition after action, whereas video recording method

reveals cognition in action. Future research on slips should focus on cognition

in action (Reed, Montgomery, Palmer, \& Pittenger, 1995). Table 1. Frequencies of different types of action slips and microslips.

Type

Capture

Redoing

Anticipation 
Hesitation

ather

Total $0.60 \quad 0.50$

$>0.40$

CII

c

$\%$

c: 0.30

CI)

$\mathrm{cr}$

f

u. 0.20 0.10 0.00 Action Slips 65310 I 16

$1-2$ months Microslips 224445746 . Action

Slips o Microslips 3-4 months Figure 1. Changes in

frequency of slip occurrence per day during the period of

Hirose, N. (2007). Towards a new taxonomy of microslips.

In S. Cumminssebree, M. A. Riley $8 \mathrm{~K}$. Shockley (Eds.),

Studies in perception and action IX. (pp. 91-94). Mahuah, N.J: Laurence Erlbaum Associates.

Joe, W. Q., Ferraro, M., \& Schwartz, M. F. (2002).

Sequencing and interleaving in routine action production. Neurocase, $8,135-150$.

Jonsdottir, M. K., Adolfsdottir, S., Cortez, R. D., Gunnarsdottir, M., \& Gustafsdottir, A. H. (2007). A diary study of action slips in healthy individuals. Clinical Neuropsychologist, $21,875-883$.

Norman, D. A. (1981). Categorization of action slips. Psychological Review, 88,1-15.

Reason, 1. T. (1984). Lapses of attention in everyday life. In R. Parasuraman \& D. R. Davies (Eds.), Varieties of attention (pp. 515-549). Orlando, FL: Academic Press. 
Reed, E. S., Montgomery, M., Palmer, C., \& Pittenger, J. (1995). Method for studying the invariant knowledge structure of action: Conceptual organization of an everyday action. American Journal of Psychology, 108, 37-65.

Reed, E. S., Palmer, C. F., \& Schoenherr, D. (2009). On the nature and significance of microslips in everyday activities. Journal of Ecological Psychology, 4, 51-66.

J. B. Wagman \& c. C. Pagano (Eds.)

(9) 2009 Taylor \& Francis Group, LLC Relationship Betueen Attention and Stepping $Y$ oshifumi Ikeda, Shogo Hirata, Hideyuki okuzumi, \& Mitsuru kokubun Tokyo Gakugei University Attention when walking was examined in a study that measured reaction

time (R $T$ ) on a probe-reaction task performed while walking on a treadmill (Ku

rosaua, 1994). This study concluded that the speed of treadmill walking at

which R T minimized approximated the speed of a preferred gait, and that the

faster or slower the walking speed, the longer RT became. According to a dy

namical systems approach (Hoyt 8Taylor, 1981), the stability of system coordi

nation is higher in the optimal mode of walking, that is, the burden of a pre

ferred gait on attention is essentially lower than any other speed of walking. It is,

therefore, well supported that RT on a probe-reaction task could minimize at a

preferred gait in the study by Kurosawa (1994). Although this finding is quite

interesting, there are few other studies th ${ }^{*}$ t concluded similarly. Therefore, this

study examines stepping, an analogy of walking, and the relation between the 
stability of the motor system and attention by assigning a probe-reaction task

and the Stroop test (Stroop, 1935) while in synchronized stepping under various

rhythm conditions. Method A total of 10 adults (4 male, 6 female) from 17 to 29 years of age

(22.90 92.88 years) participated in this study. Subjects were to perform a probe

reaction task and the stroop test, which are cognitive tasks to assess attention,

while in synchronized stepping under five rhythm conditions of $0.5 \mathrm{~Hz}, 1.0 \mathrm{~Hz}$,

$2.0 \mathrm{~Hz}, 3.0 \mathrm{~Hz}$, and $4.0 \mathrm{~Hz}$ at a fixed place. A metronome was used to make

sounds informing subjects of the rhythms. In a probe-reaction (Probe) task

which used black rectangular patches as stimuli, subjects named the color of the

black patches presented sequentially. The stroop test was composed of two

tasks: a color naming (CN) task, in which subjects named the color of patches in

red, blue, green, or yellow presented in a random order; an incongruent color

naming (1CN) task, in which subjects named the color of words printed in in

congruent colors presented in a random order. In ICN task, stimuli were four

words ('red', 'blue', 'yellow', and 'green') printed in a non-matching color of

the same four colors. All the stimuli of Probe, CN, and ICN tasks were pre

sented in the white-screened monitor in front of subjects and the reaction time 
(msec) between presentations of stimuli and oral responses in a microphone was

measured by super Lab (Cedruse). The mean time of 8 trials for Probe, $\mathrm{CN}$, and

ICN tasks in each stepping rhythm condition were used as representative subject

variables. Results and Discussion Figure 1 shows the mean ofRT on Probe, $\mathrm{CN}$, and ICN tasks for each step

ping rhythm condition. Figure 2 shous logarithmic $\mathrm{Hz}$ and RT placed on the

horizontal axis and vertical axis, respectively. In both figures, the decisive coef

ficient of the retrogressive curve was broadly high in Probe, cN, and ICN tasks.

For Probe, CN, and ICN tasks, the RT minimized at $2.0 \mathrm{~Hz}$ and extended wheth

er the $\mathrm{Hz}$ was higher or lower. Therefore, in any cognitive tasks, the change of

RT between each rhythm condition showed a V-shaped curve whose trough was

around $2.0 \mathrm{~Hz}$. In other words, the low burden on attention occurred when step

ping around $2.0 \mathrm{~Hz}$. Since it has been already clarified that changes of height

and the interval of stepping while performing minimize when stepping around

2.0 $\mathrm{Hz}$ (Kamiyama et al; submitted), the coordination and stability of stepping at

2.0 $\mathrm{Hz}$ can be even higher. Thus, it follous that a similar conclusion to Kurosa

wa (1994) applies for stepping as well. $800700^{\circ}$ !

$600 \approx 500400 \cdots 0 y=15.28 \times 177.80 x+762.5 \mathrm{Rl}$

$\left.=0.89=24.74 \times 11^{\infty} 138.59 x+752.03 y=43.97\right)(2237.29 x$

$+727.61 \mathrm{Rz}=0.73 \cdot .234 \mathrm{ll}$. Ie . Probe $\mathrm{Hz}$ 
Figure 1. Mean reaction time of cognitive tasks at each stepping rhythm. A two-way ANOYA (within subjects)

demonstrated significant effects of

the cognitive task-factor ( $F 2$, IS= $65.990, p<.00 I$ ), the stepping rhythm-factor

( $\mathrm{F} 4,36=44.749, \mathrm{p}<.00 \mathrm{I})$ and interaction effects between the cognitive task-factor

and the stepping rhythm-factor ( $F s, n=4.793, p<.00 I$ ). The significant interaction

effects suggest that the effect of rhythm conditions will not be uniform for each

cognitive task. Apparent from the relation between cognitive tasks, RT on the

Probe task was shorter than RT on the eN task, and RT on the eN task was

shorter than the les task in any rhythm condition, which would be affected by

the difference in difficulty. However, the change of RT between rhythm condi

tions in the lew task, which is thought to be the most difficult cognitive task,

was less distinct than that of the other two tasks. That is, the les task in the

Stroop test has a constant difficulty as a cognitive task irrespective of the stabil

ity of stepping. This is more than a little paradoxical considering the greater

changes in RT between rhythm conditions in the Probe and en tasks, which are

thought to be less difficult. On the other hand, from

figure $I$, the extension of

RT was not uniform whether it was in the higher or lower $\mathrm{Hz}$ with $2.0 \mathrm{~Hz}$ cen

tered. This suggests that the burdens of stepping at 
ferent from one another. The relation between the stability of stepping and the

performance on cognitive tasks has to be discussed in more detail in another

article. $=0.04 \times 20.07 x+9.42 \mathrm{R} 2=0.996$ IC OC .

Probe $21023 \mathrm{~Hz}$

Hoyt, D. \& Taylor, c. (1981). Gait and the energetics of locomotion in horses. Nature, 292, 239-240.

Kurosalua, K. (1994). Evaluation of locomotion efficiency by Probe-reaction time during treadmill walking at various speed and cadence. Journal of Kyorin Medical Society, 25, 527-536. (In Japanese with English abstract.)

Stroop, J. R. (1935). Studies of interference in serial verbal reactions. Journal of Experimental Psychology, 18 , 643-662. Acknowledgements. The authors would like to thank the participants who

made this work possible.

(c) 2009 Taylor \& Francis Group, LLC Relationship Between Stepping and its Tempo Yu Kamiyama, Shogo Hirata, Hideyuki okuzumi, \& Mitsuru Kokubun Tokyo Gakugei University

In recent years, many studies of reciprocal movement have been conducted

using the dynamical systems approach (Kelso, 1995). There are many kinds of

reciprocal movements. Sekiya et aI, (1996) focused on the human gait, which is

a representative reciprocal movement, and clarified that whether the walking

speed is fast or slow, the variability of elements, such as increasing step length

or step rate, may increase and lead to a breakdoun of gait stability. Although the

above findings of Sekiya et aI, (1996) are very interesting, few studies have been 
conducted on reciprocal movements from this vieupoint. In this study, we in

vestigate the relationship between stepping and its tempo. A synchronized step

ping movement is a similar movement to the human gait.

Method

The subjects were 30 healthy adults (15 males, 15 females) aged 20 to 29

years (22.81 \pm 1.70 years). In this study, the subjects stepping movement was

recorded by a position sensor (c2399, Harilamatsu

Photonics, Japan), consisting

of a camera and amplifier which could record the track trajectory of an LED

from a distance. The track of the LED is output laterally and sagittally. These

two analog signals uere input to a personal computer though an AID conversion

board at a sampling frequency of $100 \mathrm{~Hz}$ and recorded on a hard disk. Subjects

stand up on a flat floor wearing low-heeled shoes or thin socks and a right ankle

band to which the LED was attached, and were asked to step according to a

sound. Sound stimuli were presented by electronic metronome, and the sounds

continued while stepping. In this study, we presented five rhythm tempos such

as $0.5 \mathrm{~Hz}, \mathrm{I} \mathrm{Hz}, 2 \mathrm{~Hz}, 3 \mathrm{~Hz}$ and $4 \mathrm{~Hz}$, which were randomized for each person.

Finally, subjects were asked to step naturally without the rhythm sounds at a 
preferred tempo. Using the recorded digital data, we calculated the ten values of step time

(ms) and step height (cm) at each rhythm tempo. Then, the average values (step

time, step height) and coefficients of variation (time cV, height cV) were calcu

lated for each person. Step time is defined as the average rhythm in the stepping

movement, and can be used as index of whether the subject stepping is synchro

nized with the presented stimulus. Step height is an index that shous the maxi

mum movement amplitude in the vertical direction in right leg. The time cV and

height cV can be used as ari index of step time variability and step height varia

bility. If these two values are large, the variability of step time or step height is

high, and the stability of stepping is low.

Results and Discussion

Table 1 summarizes the mean (M) and standard deviation (SD) of each mea

surement at each rhythm tempo. In this study, we only measure the right leg

movement by the position of the sensor. Thus, the step time's theoretical value

was the time for two iterations of stimulus sound at the each rhythm tempo, such

as $0.5 \mathrm{~Hz}=4000 \mathrm{~ms}, 1 \mathrm{~Hz}=2000 \mathrm{~ms}, 2 \mathrm{~Hz}=1000 \mathrm{~ms}, 3 \mathrm{~Hz}$ $=667 \mathrm{~ms}$, and 4

$H z=500 \mathrm{~ms}$.

Table 1. Mean (M) and standard deviation (SD) of each variable at each rhythm tempo 
Rhythm step time (ms) step height (cm) Time cV Height cV

tempo $M S D M S D M S 0 M S 0$

$0.5 H z \quad 4001.17 \quad 26.09 \quad 14.78 \quad 7.17 \quad 0.05 \quad 0.02 \quad 0.18 \quad 0.11$

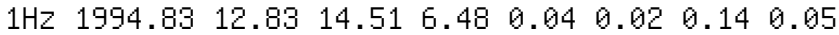

$2 \mathrm{~Hz} \quad 1000.00 \quad 5.25 \quad 9.17 \quad 4.42 \quad 0.04 \quad 0.01 \quad 0.12 \quad 0.04$

$3 H z \quad 668.50 \quad 7.67 \quad 5.63 \quad 2.85 \quad 0.06 \quad 0.02 \quad 0.15 \quad 0.05$

$4 \mathrm{~Hz} 505.67 \quad 7.76 \quad 4.63 \quad 2.23 \quad 0.08 \quad 0.02 \quad 0.17 \quad 0.06$

$\ldots \ldots \ldots \ldots \ldots-_{-}^{-} \cdots \cdots, \cdots \ldots \ldots$.

$\ldots \ldots \ldots \ldots \ldots \ldots \ldots \ldots \ldots \ldots \ldots,{ }_{11}$

$\ldots \ldots \ldots \ldots \ldots \ldots \ldots \ldots$

Prefer $1107.00188 .8210 .125 .46 \quad 0.040 .020 .110 .05$

The results show that the step times correspond to the theoretical value at

each rhythm tempo. Moreover, the preferred tempo approximates the values at 2

$\mathrm{Hz}$. Step height rises significantly with the slowing of the tempo $(\mathrm{F} 5,145=54.2, \mathrm{P}$

<. .1). Time cu becomes larger when the tempo is slouer or faster, and it is a

minimum at $1 \mathrm{~Hz}$ and $2 \mathrm{~Hz}(\mathrm{F5}, 145=27.7, \mathrm{P}<.01)$. The height cV also becomes

larger when the tempo is slower or faster, and it is a minimum at $2 \mathrm{~Hz}$ (F 5 * $145=$

$7.3, \mathrm{P}<.01)$. The change of each cv between each rhythm tempo formed a $U$

shaped curve whose trough was around $2 \mathrm{~Hz}$. Each cy of the preferred tempo

was small, and the stability of preferred stepping was very high. Results in this study were as follows. First, step time strongly correlates to

step height. This relationship between step time and step height suggests the 
possibility that one synergy (Bernstein, 1996) was formed in stepping. Second,

in the vicinity of $2 \mathrm{~Hz}$ tempo, temporal stepping stability and spatial stepping

stability were very high, whereas, when the rhythm tempo became faster or slow

than $2 \mathrm{~Hz}$, there was a breakdown of stepping stability. These results accord

with the findings of Sekiya et al. (1996). In this study, we could say that the sta

bility of preferred stepping was very high. In further studies, we will have to

examine various-aged children's performance to investigate the developmental

process of stability of stepping.

Bernstein, N. A. (1966). On Dexterity and Its Development. Mahwah, N.J: Law

rence Erlbaum Associates.

Kelso, J. A. S. (1995). Dynamic Pattens. Cambridge, MA: The MIT press.

Sekiya, N., Nagasaki, H., Ito, H., Furuna, T (1996). The invariant relationship

between step length and step rate during free walking, Journal of Human

Movement Studies, 30,241-257. (In Japanese with English abstract.) Acknowledgements. The authors would like to thank the participants who

made this work possible.

(6) 2009 Taylor \& Francis Group, LLC

Dynamics of Motor Performance in Visually Guided Force Production Nikita A. Kuznetsov \& Michael A. Riley Perceptual-Motor Dynamics Laboratory, Department of Psychology, University of Cincinnati The structure of motor variability is informative about underlying motor 
control processes (Riley \& Turvey, 2002; Slifkin \& Newell, 1999). The presence

of structured (non-random) fluctuations in movement is thought to indicate a

capacity for greater behavioral flexibility and greater overall adaptability to

novel situations compared to systems that do not exhibit this pattern of fluctua

tion. In this study we examined the structure of variability of single-digit force

production in regard to the use of adaptive motor control strategies. Producing a specified level of force could be achieved using two general

strategies. The first involves anticipatory modulations of force in order to keep

current performance within criterion levels. This is possible if the frequency of

visual feedback is high and the task goal is known, conditions that are known to

facilitate performance (Sosnoff \& Newell, 2005). The second strategy involves

utilizing feedback in a reactive, rather than prospective, fashion-force correc

tions are made only after the force criterion is violated. This strategy is expected

when information that permits prospective control is absent. The goal of this study was to determine the relation of the structure of force

fluctuations to prospective and reactive force control strategies. Participants

produced a specified force under manipulations of visual information about per

formance. A continuous display, which was designed to encourage prospective 
control, indicated the current level of force in relation to the goal and to the

boundaries of an acceptable range around the goal. A discrete feedback display,

which was designed to elicit a reactive strategy, only signalled when (and in

which direction) the current level of force deviated from the acceptable range.

Prospective control in the continuous condition was expected to yield smoother

changes in force values. In contrast, the reactive changes in force based on dis

crete feedback were expected to yield discontinuous force time series containing

accasional irregularities due to rapid force readjustments enacted when feedback

was presented. These structural differences in the force signals uere quantified

using the \&LAM measure from recurrence quantification analysis (RQA; Mar

wan, 2002; Weber \& zbilut, 2005). KLAM is the percentage of recurrent points 27

forming vertical lines in the recurrence plot. Signals with more discontinuities

exhibit a greater number oflaminar states (and hence greater sLAM) than more

smooth, cont inuous signals. Method Eleven right-handed University of Cincinnati undergraduates participated

for course credit. Participants attempted "o produce a constant level of force

(208 of the participant's maximum; mean maximum force was $37.1 \pm 8.7 \mathrm{~N})$

with two different kinds of displays continuous vs. 
discrete) and three levels of

difficulty (allowed force variability of 1,5 , and $10 \%$ of the target level). There

were 4 trials in each condition (24 trials total), each lasting 30 seconds, pre

sented in random order. Force was recorded at $100 \mathrm{~Hz}$ using a Bertec (Wor

thington, oH) load cell. Unfiltered forces normal to the load cell surface were

analyzed. Participants sat at a table with the load cell mounted on it, $40 \mathrm{~cm}$ away from

a monitor positioned at eye height. In the continuous display condition, the goal

force was indicated by a gray band on the display spanning the minimum and

maximum levels of allowed force. Current force was indicated in real time by a

red dot. Participants tried to maintain the dot within the band. They could al

ways see how the force they were producing related to the goal level. In the discrete display condition participants were asked to produce the

same levels of force, but the red dot only took three possible discrete, static val

ues: above, within, or below the band, indicating, respectively, that force ex

ceeded, matched (within limits), or was less than the goal force. Force was

measured in real time but the dot position did not change unless force changed

from one of those three states to another. . RoA was conducted using $d=3,1$ : $=20$, and a variable radius calculated as

a percentage of the mean distance between time-delayed 
vectors of force values.

Radius was adjusted to achieve gREC $=1 \pm 0.2$ for each trial. Tuo consecutive

vertical points defmed a vertical line. The first 4 seconds of the trial were dis

carded to omit transients. Results and Discussion Figure

1 shous sample force time series and corresponding

recurrence

plots. The percentage of sampled force values within the allowed limits (accu

racy) and \&LAM were calculated for each trial and averaged over trials in each

condition. These averaged values were submitted to separate 2 (display: con

tinuous vs. discrete) $\times 3$ (difficulty: 1,5 , and 108 of target force level) repeated

measures ANOV As. There was a main effect of difficulty on accuracy, $F(2,22)=990.76, P<$

$.0001,77 /=.99$. Participants were least successful in the 18 condition followed

by the $5 \%$ and $10 \%$ conditions (Figure 2, left). Participants performed better

with the continuous displays than the discrete ones, $F(1$, 22) $=49.32, p<.0001$,

$77 /=.82$-it was harder to maintain the 'required level of force using discrete

feedback. The difficulty $x$ display interaction approached but did not reach sig

nificance, $F(2,22)=3.34, P=.054,17 /=.23$.

Discrete displays led to higher \&LAMthan continuous

displays, $F(1,22)=$

102.68, $\mathrm{P}<.0001,17 /=.90$ (Figure 2, right). This

indicates a greater tendency

for discontinuous irregularities in the force time series 
condition, and suggests that \&LAM can be used to measure differences between

the qualitatively different motor control strategies encouraged by these display

conditions. Difficulty did not have an effect on \&LAM, $(\mathrm{p}=$ $.09,17 /=.20$ ), and

although differences between the two kinds of display conditions were more

pronounced in the most difficult ( 18 range) condition there was no difficulty $x$

display type interaction $(p=.42,17 /=.08)$. Future research will focus on identi

fying critical values of feedback characteristics that elicit qualitative shifts in

isometric force production strategies. Dimension : 4 . Delay: 70 . Radius 28 \& Force produced by participant 5

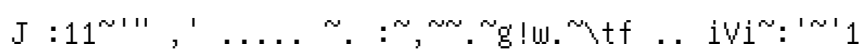

7.50510152025 Time lsI Dimension: 4. Delay: 70.

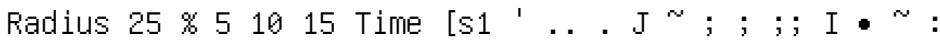

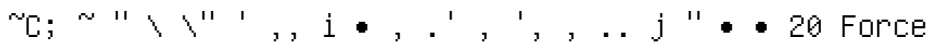
produced by participant 5 - 9.5 ts. $9 \mathrm{I},{ }^{\circ} \sim 8.5$ 'II 'f

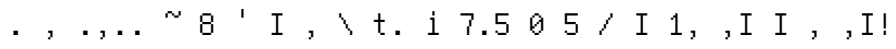
. ! Figure 1. Time series (bottom) of force produced in the I $\&$ cont inuous (left panel)

and $I \&$ discrete (right panel) displays and (top) their corresponding recurrence plots.

\&LAM is $63 \%$ in the continuous condition and $94 \%$ in the discrete condition. $1009080: ; .70 ;: 60 \sim 50 \ldots$ i: $140 \sim 30 \quad 20 \quad 10 \quad 0 \quad 8078 \quad 76 \quad 74 \sim 72: 570 \sim 68$ 66646260 , I. ---.a. , , , , 5 Allowed Variability W.) 5 Allowed Variability ..) 1010 29 Figure 2. Average values of accuracy (left) and \&LAM (right). Error bars indicate

Maruan, N., Messel, N., Meyerfeldt, U., Schirdewan, A., \& Kurths, J. (2002). Recurrence-plot-based measures of complexity and their application to heart-rate-variability 
data. Physical Review E, 66, 026702-026702.

Riley, M. A., \& Turvey, M. T. (2002). Variability and determinism in motor behavior. Journal of Motor Behavior, $34,99-125$.

Slifkin, A. B., \& Newell, K. M. (1999). Noise, information transmission, and force variability. Journal of Experimental Psychology: Human Perception \& Performance, $25,837-851$. .

Sosnoff, J. 1., Newell, K. M. (2005). Intermittent visual information and the multiple timescales of visual motor control of continuous isometric force production. Perception \& Psychophysics, 67, 335-344.

Webber, C. L., \& zbilut, 1. P. (2005). Recurrence quantification analysis of nonlinear dynamical systems. In M. A. Riley \& G. C. Van Orden (Eds.), Tutorials in contemporary nonlinear methods for the behavioral sciences (pp. 26-95). Retrieved 0ctober 27, 2008, from http://ululu. nsf . gow/sbe/bcs/pac/nmbs/nmbs. jsp.

(9 2009 Taylor \& Francis Group, LLC Microslips in a Manual Placing Task Ken Yoshida \& Thomas A. Stoffregen University of Minnesota Microlips are small glitches that occur in manual action. Previous research

has documented the existence of microslips in adults and children (e.g., Reed 8

Schoenherr, 1992, Suzuki \& Sasaki, 2001). These authors argued that micros

lips occur during transitions between one action to another. Suzuki \& Sasaki

manipulated the complexity of the task situation (e.g., the presence or absence of

distracter elements) and found that microslips were more common in more com

plex task situations. The task complexity manipulations of Suzuki and Sasaki (2001) were quali

tative. We attempted to develop a method in which the frequency of micros lips

could be related to quantitative manipulation of a control 
ined two preferred modes in manual, pronation and supination. Preferred modes

of action have been widely studied including grip configuration (Cesari \& New

ell, 1999), intraand inter-personal grasping (Richardson, et aI., 2007), and bi

manual fmger coordination (Kelso, 1984). We asked participants to grasp a

wooden rod and place it upright on a table. To do this, they were obliged to

choose between pronate and supinate hand. posture in grasping the rod. We var

ied the initial position of the rod, in terms of its angle relative to the participant.

We hypothesized that the occurrence ofpronatory or supinatory reaching would

be influenced by the angle of the rod, and that at some critical angle there would

be a reliable boundary between pronate and supinate reaching. We expected

microslips to occur as participants prepared to grasp the rod, and we predicted

that microslips would be more common when the rod angle was near the transi

tion between pronate and supinate reaching modes. Method Tuenty-one undergraduate students participated. The experimental protocol

was revieued and approval by the institutional review board of the University of

Minnesota. Participants sat in front of the table, with a rod presentation device on either

side (Figure 1). The angle of rod was varied, in 150 increments, from -150 to 
$90^{\circ}$. Participants were asked to pull the rod from the rod-base and place it verti

cally on the table in front of them. There were 80 trials per participant (40 for

each hand), which were video recorded. Frequencies of pronation/supination reaching and microslips were coded

from the video files. In addition, microslips were categorized using the classifi

cation of Reed and Schoenherr (1992). The categories were hesitations, trajecto

$r y$ changes, touches, and hand shape changes. Figure I. Experimental setting. Two rod-bases were placed on right and left sides of

the table. Angles of rod were adjustable with $15^{\circ}$ increments. Participants were in

structed to lift and place the rod on the target which was marked on the table. Results and Discussion contrary to our expectation, rod angle had only a weak effect on prona

tion/supination. This was because participants elected to use supination in

99.98 of cases when reaching for the rod. The rare pronate reaches tended to

occur when the rod angle was $-15^{\circ}$. Thus, we were not able to test our predic

tion that microslips uould occur near pronation/supination transitions. There

were a total of seven microslips in the reach/grasp phase. six of these were

touches, and one was a hand shape change. Dur analysis revealed that micros lips were more common in the terminal

part of the action, when participants were preparing to place the rod on the table.

We observed 29 hesitations during rod placement. This result indicates that 
microslips can occur in relatively simple situations. Reed and Schoenherr (1992) argued that microslips should tend to occur

near transitions of action modes. This insight inspired our attempt to develop a

method in which the frequency of microslip occurrence would be related to

quantitative manipulation of a control parameter that was related to a transition

between two action modes. We developed a grasp-and-place task in which par

ticipants were required to choose between pronation and supination of the hand

when reaching for the to-be-grasped object. The utility of our method depended

upon the occurrence of a transition between pronation and supination. We

found some evidence of a transition between reaching modes, but this evidence

occurred only at one of the extreme values of our control parameter. In future

research, we will attempt to identify values of a control parameter that can relia

Cesari, P., \& Newell, K M. (1999). The scaling of human grip configurations. Journal of Experimental Psychology: Human Perception and Performance, 25, 927-935.

Kelso, J. A. S. (1984) Phase transitions and critical behavior in human bimanual coordination. American Jounral 0 Physiology: Regulatory, Intergrative and Comparative, 15, RI000-R1004.

Reed, E. S., \& Schoenherr, D. (1992). The neuropathology of everyday life: On the nature and significance of micro-slips in everyday activities. Unpublished manuscript.

Richardson, M. J., Marsh, K L, \& Baron, R. M. (2007). Judging and actualizing intrapersonal and interpersonal 
affordances. Journal of Experimental Psychology: Human Perception and Performance. 33, 845-859

Suzuki, K, \& Sasaki, M. (2001). The task constraints on selection of potential units of action: An Analysis ofmicrosjips observed in everyday tasks. Cognitivestudies, 8,121-138. 


\section{Chapter 2: Interpersonal Coordination}

Isenhower, R. W., Marsh, K. L., Carello, c., Baron, R. M., \& Richardson, M. J. (2005). The specificity of intrapersonal and interpersonal affordance

boundaries: Intrinsic versus extrinsic metrics. In $\mathrm{H}$. Heft \& K. L. Marsh

(Eds.), Studies in perception and action, viii. Mahwah, N.J: Er lbaum.

Warren, W. H., \& whang, S. (1987). Visual guidance of walking through

apertures: Body-scaled information for affordances. Journal of Experimental Psychology: Human Perception and Performance, 13,371

383.

(6) 2009 Taylor \& Francis Group, LLC

Interand Intra-personal Coordination in Autistic and Typically-Developing Children Robert $w$. Isenhouer!, Kerry L. Marsh!, Paula Silva l, Michael J. Richardson2, and R. C. Schmide iCenter for the Ecological Study of Perception and Action, University of connecticut 2Department of Psychology, Colby College 3Department of Psychology, College oftbe Holy cross Autism Spectrum Disorder (ASD) is a pervasive developmental disorder

that is currently diagnosed on the basis of a triad of behavioral indicators: (1)

deficits in social interaction, (2) deficits in language for communication, and (3)

restricted and repetitive interests and activities (DSM-IV-TR, 2000). Points (1)

and (2) are fundamentally coordinative in nature. Therefore, studying coordina

tion in children with ASD may reveal subtleties of the dynamics of intrapersonal

and interpersonal coordination not apparent in typically-developing (TD) child 
ren. Coordinated behavior has been shown to be constrained by a coupled oscil

lator dynamic and can be described by the so-called HKB equation (Haken, Kel

so, \& Bunz, 1985): . ; = $\cdots$ a sm ; 2 b $\sin 2 ;+\$$;

Predictions of the HKB equation have found substantial support in a pendu

lum-swinging paradigm. This is so whether the pendulums are held by a single

individual (Kugler \& Turvey, 1987) or by. two individuals (Schmidt \& Turvey,

1994). Moreover, interpersonal coordination is apparent even without explicit

instruction to coordinate (Richardson, Marsh, \& Schmidt, 2005). Critical to the

current study, the HKB equation is not limited to the ure ist-pendulum paradigm.

The current study uses an interpersonal rocking chair paradigm (cf. Richardson,

Marsh, Isenhouer, Goodman, \& Schmidt, 2007) and an intrapersonal drumming

task (cf. Brakke, Fragaszy, Simpson, Hoy, \&

Cummins-Sebree, 2007) to assess

coordination in $\mathrm{ASD}$ and $\mathrm{TD}$ children. 41 Method children (ranging from 2.5 to 8 years of age) participated in two tasks: in

terpersonal rocking and intrapersonal drumming. Dut of the total sample of

children $(N=23)$, TD children $(N m=7)$ and children with ASD (NASD $=7$ )

were age-matched based on a battery of standard clinical assessments. During the interpersonal task child/parent pairs were seated in rocking

chairs (Fig. 1, left). Movement data were collected from sensors attached to the 
chairs. The parent's rocking chair was weighted so that its natural frequency

closely matched the natural frequency of the child's rocking chair (;:; $0.83 \mathrm{~Hz})$. A

metronome that only the parent could hear was used in order to keep the rocking

frequency of the parent constant. This also ensured that any spontaneous coordi

nation observed was due to the child entraining to the parent. Parents read a

story to their child in order to keep their attention. children were never told to

rock; however, most children did rock spontaneously. Figure 1. (left) Interpersonal rocking and (right) intrapersonal drumming. During the intrapersonal task, children were first shown how to hold the

drumsticks and how to strike the drum in an in-phase and an anti-phase manner.

They were then instructed to strike the drum continuously for two 45 second

trials (one in-phase and one anti-phase) at their oun preferred pace. The order of

the trials was counterbalanced across participants.

Movement data were col

lected from sensors attached to the drumsticks (Fig. 1, right). Results and Discussion for interpersonal rocking, continuous relative phase (CRP) was examined.

The amount of time that the child's chair "as in a particular phase relation with

the parent, in nine steps from 0. (in-phase) to 180 .

(anti-phase), was assessed for

the age-matched sub-sample $(N=14)$ of the children. A 2 (Group: TD vs. ASD)

by 9 (Phase bins) mixed ANOYA revealed a significant Group 
$x$ Phase bin in

teraction, $F(8,96)=2.57, \mathrm{p}<.05,7) /=.18$. The CRP results for the interper

sonal rocking task indicate the pattern of coordination observed for TD children

is different than the pattern observed for children with ASD. TD children exhibit

more in-phase coordination than children with ASD; the latter showed no dis

cernable preferred phase region (Fig. 2, left). o II 5010

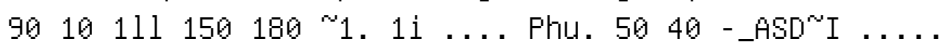

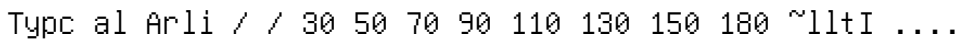
Phi .. Figure 2. (left) Amount of time spent by each group in each of the phase bins.

(right) Amount of time spent in the required phase (inor ant $i-p h a s e)$ by the two groups

of children. For intrapersonal drumming, CRP was examined separately for in-phase

coordination and for anti-phase coordination using parallel Group $x$ Phase re

gion mixed ANOV As for the age-matched sub-sample $(\mathrm{N}=14)$. The analyses

revealed a significant Group $x$ Phase bin interaction for both. For in-phase coor

dination, $\mathrm{F}(8,96)=3.81, \mathrm{P}<.001,7) /=.20$, an independent samples t-test

found a significant difference between the in-phase bin of the two groups, $t(12)$

$=2.28, p<.05$. For anti-phase coordination, $F(8,96)=$ $4.23, \mathrm{P}(.001,7) /=$

.21 , an independent samples t-test found a significant difference between the

anti-phase bin of the two groups, $t(12)=2.59, P<.05$. overall, the CRP analy 
ses of the drumming data revealed significantly more in-phase and anti-phase

coordination for TD children than for children with ASD.

Typical children are

better able to maintain the required phase during drumming (Fig. 2, right). Cross recurrence quant ification (CRD) analysis was also applied to the

rocking and drumming data. CRQ measures the dynamic similarity between two

time series embedded in a reconstructed phase space. No significant effects were

found for rocking. For drumming, houever, differences were apparent. For anti

phase coordination, TD children had significantly greater $\%$ recurrence than

ASD children, $\mathrm{t}(12)=2.29, \mathrm{p}<.05$, signi(icant ly longer meanline, $t(12)=3.14$,

$\mathrm{P}<.05$, and a marginally longer maxline, $\mathrm{t}(12)=1.82, \mathrm{P}<$ .08. For in-phase

coordination, in contrast, TD children had a marginally shorter mean line than

ASD children, $t(12)=2.12, \mathrm{P}<.06$. Previous research has shown that for CRQ

analysis maxline and meanline are indices of attractor strength and 8 recurrence

is an index of noise (Richardson, schmidt, \& Kay, 2007). under this analysis, it

is possible that typically developing children assemble oscillatory drumming

movements that have less noise than children with ASD. However, given that

the mean line difference between TD children and children with ASD is the op

posite for in-phase coordination and anti-phase 
coordination, the results con

cerning attractor strength of the drumming movements are equivocal. overall, the results of the current study find coordination differences be

tween children with $A S D$ and TD children for both interpersonal and intraper

sonal tasks. Uninstructed interpersonal coordination between parent and child in

the rocking task revealed that TD children spent more time in phase with the

parent whereas ASD children showed no particular preferred phase relation. For

the intrapersonal drumming task, which required maintaining a particular phase

relation between their oun hands, both groups of children were successful: 0'

was produced under the in-phase instruction, 180' was produced under the ant $i$

phase instruction. However, TD children were better at maintaining the required

phase relation. The results of $\mathrm{CRQ}$ analysis indicate that TD children assemble

oscillatory movements with less noise than children with ASD, although it is

unclear if the attractor strength of the oscillatory movements in either group

American Psychiatric Association. (2000). Diagnostic and statistical manual of mental disorders: DSM-IV-TR. Washington, DC: American Psychiatric Press, Inc.

Brakke, K., Fragaszy, D. M., Simpson, K, Hoy, E., \& Cummins-Sebree, $s .(2007)$. The production of bimanual. percussion in 12to 24 -month old children. Infant Behavior 8 Development, $30,2-15$.

Haken, H., Kelso, J. A. S., \& Bunz, H. (1985). A theoretical model of phase transitions in human hand 
movements. Biological cybernetics, 51, 347-356.

Kugler, P. N., \& Turvey, M. T. (1987). Information, natural law, and the selfassembly of rhythmic movements. Hillsdale, N.J: Laurence Erlbaum Associates.

Richardson, M. 1., Marsh, KL., Isenhower, R. W., Goodman, 1., \& Schmidt, R. C. (2007). Rocking together: Dynamics of intentional and unintentional interpersonal coordination. Human Mov?menf Science, 26, 867-891.

Richardson, M. J., Marsh, K. L., \& Schmidt, R. C. (2005). Effects of visual and verbal information on unintentional interpersonal coordination. Journal of Experimental Psychology: Human Perception and Performance, 31, 62-79.

Richardson, M. J., Schmidt, R. C., \& Kay, B. A. (2007). Distinguishing the noise and attractor strength of coordinated limb movements using recurrence analysis. Biological cybernetics, 96, 59-78.

Schmidt, R. C., \& Turvey, M. T. (1994). Phase-entrainment dynamics of visually coupled rhythmic movements. Biological Cybernetics, $70,369-376$.

Acknowledgements. This research was supported by a cure Aut ism Now

(CAN) Grant and a National Science Foundation Grant \# 0240277 .

(9) 2009 Taylor \& Francis Group, LLC The Influence of Interpersonal Interaction on Postural Coordination Dynamics in a Suprapostural Task

Manuel Var let, Ludovic Marin, Julien Lagarde, \& Benoit G. Bardy

Efficiency and Deficiency Laboratory, University Montpellierl, Montpellier, France Previous postural coordination studies have shoun that multi-segment post

ural coordination can be described by the relative phase between the ankle and

hip in a suprapostural task (i.e., when posture is investigated within a goal

directed task; Bardy, Marin, Stoffregen, \& Bootsma, 1999). Research revealed 
that when participants "posturaly" track the sinusoidal

motion of a target, two

stable patterns emerge from the ankle-hip relative phase: an in-phase mode

(close to $20^{\circ}$ ) for low target frequency displacements and an ant i-phase mode

(180 ) for high target frequency motions. Increasing target frequency produced

an abrupt change from in-phase to anti-phase coordination and inversely, de

creasing frequency produced a change froin anti-phase to in-phase coordination

(Bardy, Dullier, Bootsma, \& Stoffregen, 2002). For a decade, studies have been investigating postural

coordination when

participants were alone and almost none have examined posture in interpersonal

situations. In our everyday life, however, posture it is not an end in itself, but

rather it is often used to (involuntarily) communicate, work with someone, prac

tice a sport and so on. Shockley, Santana and Fowler (2003) have shoun objec

tive evidence of postural influence in interpersonal situation. However, this

study only measured a global assessment of postural activity and did not investi

gate the postural coordination of paired participants. The goal of the current

study, therefore, was to investigate whether postural coordination can be influ

enced during a visual interpersonal interaction. Based on previous research on 
postural coordination (Bardy et aI., 1999; 2002) we know that in order to per

form a suprapostural task, participants modify their postural coordination. They

adapt the ankle-hip relative phase to the situation they are involved in. Conse

quently, we hypothesized that in interpersonal situations participants would

adapt their oun ankle-hip relative phase to be coordinated with their co-actor. Method Twenty participants stood in front of a target simulated on a monitor and

were instructed to track the A-P oscillations of the target with an increase and a

decrease of frequency ranging from $0.10 \mathrm{~Hz}$ to $0.75 \mathrm{~Hz}$ with step of $0.05 \mathrm{~Hz}$ (see

Bardy et al. 2002 for more details). No instructions were given on the postural

coordination they could adopt. They performed the tracking task in two condi

tions (Figure 1): paired (Duo condition) and alone (Solo condition).

A B Figure 1. In the Duo condition ( $A)$ participants saw their co-actor in their peripheral

view. In the solo condition (B) participants wer.e alone to perform the tracking task. To analyze the influence of the interpersonal interaction on the spontaneous

postural coordination dynamic, three variables were computed: I) distribution

and 2) standard deviation of the ankle-hip relative phase of each participant,

indicating the coordination modes produced and their stability; 3) absolute dif

ference between the postural transition frequencies (TF) of each paired partici 
pant in order to determine the influence of interpersonal interaction on the tran

sition frequency. Results and Discussion I) The distribution of ankle-hip relative phase angles across nine phase re

gions was submitted to a 2 (Condition) $\times 9$ (Phase Region) repeated measures

ANOV A. Using the Greenhouse-Geisser correction, this analysis yielded a sig

nificant main effect of phase region $(F(8,152)=63.26, p$ <. .05) and no signifi

cant interaction between phase region and condition ( $\mathrm{F}(\mathrm{B}$, 152) $=0.20, P>.05)$.

These results shoued that 1) anti-phase (and in-phase) coordination mostly

emerged from this task and 2) there were no postural coordination differences in

Duo and Solo conditions (Figure 2). GlobaJly, participants did not produce more

in-phase or anti-phase pattern during interpersonal interaction. $8060402000 \mathrm{~N} .0 \sim 2000$

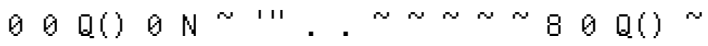

.... .... Ankle-Hip Relative Phase Region e) ด Q()

... $\theta^{\prime \prime}$-Duo a Solo Figure 2. Distribution of

ankle-hip relative phase angles for Duo (black) and Solo

(white) conditions. 2) The standard deviation of ankle-hip relative phase was $8.24(\mathrm{SD}=4.36)$

in Duo condition and $8.56(\mathrm{SO}=5.44)$ in solo condition. No difference in post

ural coordination stability was observed between Duo and Solo conditions. In

terpersonal interaction did not affect the stability of postural coordination pat

terns produced by participants. This result suggests that the strength of the 
coupling of postural coordination is strong enough to not be influenced by the

presence of the other participant. This is consistent with previous interpersonal

coordination studies and indicates that intrapersonal coordination is more stable

than visual interpersonal coordination (e.g., Schmidt et aI., 1998). 3) The mean of the absolute difference between the transition frequencies

(TF) was $0.19 \mathrm{~Hz}(\mathrm{SD}=0.14)$ for Duo condition and $0.24 \mathrm{~Hz}$ $(S D=0.14)$ for

Solo condition. One factor ANOVA with repeated measures showed significant

difference between these two conditions $(F(1,8)=10.78 p<$ .05) (Figure 3 Top),

revealing that when paired, participants spontaneously modified the frequency

of their postural transition from one coordination mode to the other. Globally

this result shows that during visual interaction, participants shifted postural

coordination patterns either earlier or later than when alone, in order to adopt a

similar postural pattern as the one performed by their co-actor (Figure 3 Bot

tom). In conclusion, this study reveals that the exchange of visual information be

tween two people can directly influence their postural coordination dynamic.

This study can also be considered as a new paradigm in investigating postural

coordination in a more social (and ecological) situation. $3^{\prime} 0.30$ c $i=0.25 M 0.20 .8 \sim 0.15$ E g $0.10 \ldots, u$

$\sim 0.05^{\prime \prime \prime}<0.00$. condit ion uo c ndition I'oni

ipa0l I I'llni ipant 247 
Figure 3. (Top) Absolute difference between TF in Duo and Solo conditions. (Bot

tom) Ankle-hip relative phase of two participants in Duo and solo conditions illustrating

that the presence of someone influenced participant's postural transition.

Bardy, B.G., Marin, L. , Stoffregen, T.A. , \& Bootsma, R.T. (1999). Postural

coordination modes considered as emergent phenomena.

Journal of Expe

rimental Psychology: Human Perceptton and Performance, 25, $1284-1301$.

Bardy, B.G., Dullier, o., Bootsma, RJ., \& Stoffregen, T.A. (2002). Dynamics of

human postural transitions. Journal of Experimental

Psychology: Human

Perception and Performance, 28,499-514.

Schmidt, R.c., Bienvenu, M., Fitzpatrick, P.A., \& Amazeen, P.G. (1998). A

comparison of intraand interpersonal coordination:

Coordination break

downs and coupling strength. Journal of Experimental

Psychology: Human

Perception and Performance, 24,884-900.

Shockley, K., Santana, M.V., \& Fowler, C.A. (2003). Mutual interpersonal

postural constraints are involved in cooperative conversation. Journal of

Experimental Psychology: Human Perception and Performance, 29,326

332. .

Acknowledgements. This research was supported by SKILLS, an 
Project (1ST contract H035005) of the Commission of the European Community.

1. B. Wagman \& c. C. Pagano (Eds.)

(9) 2009 Taylor \& Francis Group, LLC Gaze Coordination between Car orivers and Passengers: An observation Hiroshi Inou 1 , Mamoru Sawada 1 \& Hiroyuki Mishima 2 IDENSO Corporation, Japan, ZWaseda University, Japan Passengers riding in a vehicle operated by an unskilled driver (e.g., a newly

licensed driver) may feel fear or anxiety. One possible reason for such feelings

is the rough operation and consequent rough movement of the vehicle. Houev

er, there seems to be an underlying perceptual cause as well. Consider the fol

lowing two empirical facts: (1) an unskilled driver tends to focus their gaze on

the near side of his/her front view while driving and (2) a passenger (riding in a

vehicle with an unskilled driver), may be unconsciously induced to gaze at the

near side of his/her front view relative to a distant landscape, although this is not

the case when a skilled driver operates the vehicle. Why might this kind of gaze

entrainment between a driver and a passenger occur? we propose that the struc

ture of global optic flow presented to a passenger (or the passenger's visual sys

tem) is lawfully affected by a driver's "p'erceptual"

skill (or the driver's gaze

control, which could be considered as a reflection of

his/her perceptual explora 
tion) because the driver's operations that lawfully

constrain the optic flowexpe

rienced in the vehicle must be controlled by the driver's perception. Therefore,

when a driver's skill is poor, a passenger's gaze movement might be unstable,

and he/she would feel uneasy. As the first step to addressing this gaze entrain

ment problem, we conducted an in-vehicle experiment.

Method The experiment was conducted at a test track in the DENSO Abashiri Test

Center (Hokkaido, Japan). The participants were: one skilled driver, who had

been employed as a test driver at DENSO Corporation for 15 years, and one or

dinary driver. In the "skilled driver condition," the skilled driver operated the

test vehicle and the ordinary driver rode along in the passenger seat; in the "or

dinary driver condition," the positions were reversed. The participants' gaze

directions relative to the car's orientation were measured by recording their eye

movements at $20 \mathrm{~Hz}$ with an eye tracking system (NAC Image Technology,

EMR-8B). The movement data were smoothed using a Kalman filter. Tuo suc49

cessive curves, "Comer A" (right-hand curve; radius = 100 m) and "Comer B"

(left-hand curve; radius $=120 \mathrm{~m}$ ), were defined as the experimental trials. Both

the participants were asked to approach the first comer with an approximate 
velocity of $60 \mathrm{~km} / \mathrm{h}$ while driving and look ahead when riding as the passenger.
(a) 0.80 .6

I 0.4

2

$\sim 0.2$ Comer A RighI Driver: Skilled Driver Passenger: ordinary Driver

$\sim 0-f-\cdots, \cdot,{ }^{\sim} r-\cdots, \cdots-\cdots$.

a

$\sim 0.2$

1) $\cdot 0.4$ Left $\cdot 0.6$

(b) $0.8 \quad 0.6$

10.4

Z

0 0.2 Comer A 1015 TIME (5) Driver: Ordinary Driver Driver $\mathrm{s}$

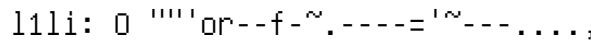

a

$\sim \sim .2 \sim 15 \sim .4$ Left $\sim .6$ TIME (5) 0.80 .6 -a $S \quad 0.4$

200.2 \& l11i: $00 \sim 0.2 \backslash 1) \cdot 0.4 \cdot 0.60 .80 .6$

! $0.4 \mathrm{Z}$. E $0.2 \sim$ is $\ldots \sim \sim .2 \cdot 0.4 \cdot 0.6$ Corner $s$

Driver: Skilled oriver TIME (5) Cotner $S$ Driver:

Ordinary Driver TIME (5) Figure 1. Gaze direction

relative to the car's orientation as a function of time:

(a)

skilled driver condition; (b) unskilled driver condition

Results and Discussion 1515 The participants' gaze

directions relative to the car's orientation in the

skilled driver and ordinary driver conditions are shown in

Figure 1. This figure

illustrates the tendency for coordination between a 
driver's and passenger's gaze

directions. Moreover, this coordination tendency appears to be stronger in the

skilled driver condition than in the ordinary driver condition. To examine the degree of gaze coordination between the driver and passen

ger, we used cross-recurrence quantificat lon analysis (CRQA; Maruan, N. , 8

Kurths, 2002; Maruan, 2003; Shockley, 2005; zbilut \& Webber, 1992). In this

analysis, the following CRQA parameter settings were used: embedding dimen

sion $=6$, delay $=0.25 \mathrm{~s}$, radius $=0.15$ radian, norm $=$ Euclidean. The data used

in the CRQA were not normalized.

(a) $12^{\prime} 010080$

$\mathrm{S}$

$\sim 60$

! Il

'I" 20406080 Driver

(b) $160 ;\left[{ }^{\sim m} \operatorname{er}^{\sim} 14011201 \mathrm{j}\right.$

$\sim 100 i$

$\sim \operatorname{soi} \theta: 6040200050100$ Dover '00 120150

$200180160 \ldots 60$ : - - , oL-.-.-...........

50100150 oriver ::: r [“rner e.J ! $16^{\circ} 114^{\circ} 1^{*}$ !zof

$\sim I^{\prime} \pm 1008060^{\prime}+20040^{\prime}$ I $2 i \ldots \ldots$

-_... - - ..J o 50100150200 Figure 2, CRPS of the

gaze directions of the driver and passenger in the ordinary

driver condition: (a) skilled driver condition; (b) unskilled driver condition The cross-recurrence plots (CRPs) for each data pair in each condition are

shoun in Figure 2. The grecurrence in each CRP is as follows: 0.104 and 0.08 
for Comers $A$ and $B$, respectively, in the skilled driver condition; 0.05 and 0.002

for Comers A and B, respectively, in the ordinary driver condition. These re

sults support the strong coordination tendency in the skilled driver condition and

the weak coordination tendency in the ord4'lary driver condition. A driver's skill is directly reflected by the vehicle's behavior. Frequent

changes in acceleration caused by poor operation of the vehicle by an unskilled

driver produce disturbances in not only the driver's and passenger's bodies but

also the optic flow field in front of the vehicle. On the other hand, the smooth

and dexterous operations by a skilled driver will prevent such vibrations, main

tain a dynamically stable optic flow, and' guide the passenger's as well as the

driver's gaze to an adequate point in the field of view. In future studies, we plan

to explore the specific optic flow information that guides drivers' and passen

gers' gaze movements.

Marwan, N. (2003). Encounters with neighbors: Current developments of concepts based on recurrence plots and their applications. Doctoral thesis, University of Potsdam, Potsdam, Germany.

Marwan, N., \& Kurths, 1. (2002). Nonlinear analysis of bivariate data with cross recurrence plots. Physics Letters A, 302(5-6), 299-307.

Shockley, K. (2005). Cross recurrence quant ification of interpersonal postural activity. In M. A. Riley \& G. C. van orden (Eds.), Tutorials in contemporary nonlinear methods for the behavioral sciences (pp. 142-177). 
Retrieved 0ctober 13,2006, from http://umu .nsf.gow/ sbe/bcs/pac/nmbs/nmbsj sp

Zbilut, J. P., \& Webber, C. L., Jr. (1992). Embeddings and delays as derived from quantification of recurrence plots. Physics Letters A, 171, 199-203.

(9) 2009 Taylor \& Francis Group, LLC Target Distance Influences Interpersonal Postural Coordination

Alison Smith 1 , Christie Pelzer 1 , Russ Gi veans 1 , Kevin Shocldey2, \& Thomas A. Stoffregen 1 luniversity of Minnesota, zuniversity of Cincinnati when pairs of people converse while standing, their body sway exhibits

spontaneous coordination (Shockley et aI., 2003). When each member of a

dyad converses with a different person, sway within the dyad is not coordinated,

indicating that the effect is related to the interpersonal interaction. The strength

of interpersonal coupling can be influenced by variations in the conversational

interaction (Shockley et aI., 2007). In a*dition, coordination might be influ

enced by characteristics of the targets of the conversation. For example, when

dyads converse about something that they can see, postural coordination might

be influenced by adjustments to posture to facilitate looking at objects of mutual

interest. Stoffregen et aI. (1999) found that the magnitude of spontaneous body suay

(in individuals) was related to the distance of visual targets: Suay was reduced

when people fixated nearby targets, relative to suay during fixation of distant

targets. This effect was functional in the sense that a given magnitude of suay 
would tend to have a more disruptive effect on fixation of nearby targets. We evaluated whether distance-related effects of vision on stance in indi

viduals might also affect the coordination of posture between members of a con

versing dyad. To test this possibility, we replicated the task partner manipula

tion in the facing away condition from shockley, et aI. (2003), and added a ma

nipulation of the distance of visual targets. We predicted that target distance

would influence the suay of individuals in each dyad, and that target distance

would influence the strength of interpersonal postural coordination. Method Thirty students at the University of Minnesota participated in return for

course credit. Participants were organized into 15 conversational pairs. We used the picture puzzle task employed by shockley et aI. (2003), using

the same cartoon pictures as that study. Within pairs, pictures were generally

similar in appearance to one another with 10 subtle differences. In the Near con

dition, the cartoon pictures were $14.0 \times 10.2 \mathrm{~cm}$ and were placed $0.5 \mathrm{~m}$ from the

participants. In the far condition, the same pictures were enlarged to $69.9 x$

$47.0 \mathrm{~cm}$ and were placed $2.5 \mathrm{~m}$ from the participants to achieve the same visual

angle as the Near condition. Members of each dyad stood facing away from

each other, on opposite sides of the emitter of a magnetic tracking system (Pol

hemus). Sensors were placed on the head (using an elastic 
headband) and hip

(using tape) of each participant, and were sampled at 30 $\mathrm{Hz}$. There were four

trials per condition yielding 16 trials per participant pair. Participant pairs were

asked to discuss their respective pictures to identify the 10 differences between

the pictures. Participants either discussed their pictures with the other participant

(Together) or with an experimental confederate (Confederate). We used a $2 \times 2$ design, with Target Distance (Far vs. Near) and Conversa

tional Partner (Together vs. Confederate), and four trials (per dyad) in each con

dition. Each trial was $120 \mathrm{~s}$ in duration. The tuo time series for a given pair for

a given body location (head vs. hip) were submitted to a cross-recurrence analy

sis (see shockley et aI., 2003 for a more detailed discussion of this analysis).

Mean \&REC (the 8 of body configurations shared by a participant pair) and

MAXLINE (the length of the longest consecutive sequence of recurring points

for a given pair) for a given condition for a given body location for each partici

pant pair were submitted to a $2 \times 2$ analysis of variance (ANOY A). Individual

participants' positional data for head and hip body locations were also submitted

to a $\times 2$ ANoYA. Results and Discussion

Cross-recurrence analysis. There were no significant effects in the 
MAXLINE variable. For the RRECUR variable the main effect of Partner was

not significant for the head or the hips. Thus, we failed to replicate the effect of

conversational partner on interpersonal postural coordination (Shockley et aI.

$2003,2007)$, though as illustrated in Figure 1 the data shoued the same trend as

Shockley et aI. (2003). However, for head motion the main effect of target dis

tance on gRECUR was significant, $F(I, 14)=5.35, P<.05$. As predicted,

variations in the visual task (target distance) influenced the strength of interper

sonal postural coupling. Participants showed less shared postural activity at the

head for far targets as compared to near targets. The data for head motion are

summarized in Figure $I$.

a:

$:: \mathrm{J}$

( $J$ \& RECuR Head Near VS. Far - Far Target o Near

Target $4{ }^{\infty}-n^{-\ldots-n}$

3+-

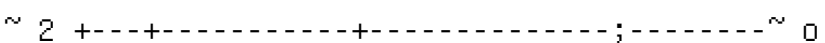

Confederate Together Confederate Together Partner Figure 1. Results of cross-recurrence analysis, showing gRECuR for dyadic head

movements as a function of conditions. Positional variability. For the head, the mean variability of position in the

$M \mathrm{~L}$ axis was $1.30 \mathrm{~cm}$ in the Near condition, and $1.58 \mathrm{~cm}$ in the far condition,

$F(1,29)=14.737, p<.01$. For the hips, the ML means 
were $0.75 \mathrm{~cm}$ and 0.91

cm, respectively, $F(1,29)=11.173, p<.01$. These effects replicate stoffregen et

al. (1999), and confirm our prediction. However, in the AP axis the influence of

target distance on head movement was in the opposite direction (MeanNear $=2.02$

$c \mathrm{~m}$, Meanpar $=1.77 \mathrm{~cm}), F(1,29)=8.46, p<.01$. We also found a significant effect of conversational partner on the position

al variability of the head in the AP axis (MeanTogether = 1. $76 \mathrm{~cm}$; MeanConfederate =

$2.03 \mathrm{~cm}), F(1,29)=5.47, p<.01$. A similar effect was observed by stoffregen,

Giveans, Villard, Yank, \& Shockley (2009), and indicates that interpersonal

influences on postural control are not limited to the dependent variables that

emerge from cross-recurrence analysis. Shockley et al. (2003) did not find a visual influence of the other participant

on postural coordination. By contrast, in the present study we did find a visual

influence of the target distance. Taken together, the results of our analysis of

positional variability suggest that participants controlled AP body movement

with respect to each other, and simultaneously controlled NL body movement

with respect to the visual targets. Comparison of our two types of analysis

(cross-recurrence and positional variability) suggest that the distance of visual

targets influenced both the sway of individuals and the 
coupling of sway within

dyads. One explanation for this pattern of results is that participants may have

exhibited greater amplitude of eye movement in the Near condition as compared

to the far condition resulting in greater postural suay in the A-P direction (cf.

Stoffregen, Bardy, Bonnet, \& Pagulayan, 2006). This could be evaluated by

tracking the eye movement patterns of participants in future studies. 


\section{Chapter 3: Perception \& Perceptual Learning}

David Katz (192511989). The world of touch. Er lbaum. Translation of Der Aufbau der Tastwelt, Leipzig: Barth.; edited and translated by Lester E. Krueger.

Acknowledgements. The first author of this study was supported by a $\mathrm{Na}$

tional Institute of Informatics internal research grant from 2007 through 2008.

(9 2009 Taylor \& Francis Group, LLC Integration of Vi suo-Haptic Information for Judging object Size Chia-Hao Lu, Joshua Arnan, \& JUergen Konczak Human Sensorimotor Control Laboratory, School of Kinesiology, University of Minnesota, Minneapolis, MN, U.S.A. Humans rout inely rely on visual and haptic information for recognizing and

manipulating objects in the environment. In trying to understand how haptic and

visual information are integrated, researchers have elucidated the relative contri

bution of visual and haptic sense by introducing visual-haptic discrepancies ex

perimentally. Previous studies on judging vertical object size showed that the visuo-haptic

integration occurs in a statistically optimal fashion by following a maximum

likelihood function (Ernst \& Banks, 2002; Gori, Del Viva, Sandini \& Burr,

2008; Helbig \& Ernst, 2008). Their fmdings also indicated that integrating re

dundant visual and haptic information of the same object improves the accuracy

of the size estimate when comparing the' size of two objects. However, these

studies did not address the issue of how the order of presenting visual or haptic

information influences the sensitivity of size perception 
when asked to discrimi

nate between two objects. In this psychophysical study, we determined the just noticeable difference

thresholds for vertical size judgment. In a first set of experiments the thresholds

were determined on the basis that only one modality was available (vision only

or haptic only). In a second set of experiments the stimulus presentation order

(visual or haptic or simultaneous) was altered to determine the relative contribu

tion of each sense for size perception as a function of when the information be

came available. Methods Healthy college level students

(aged 1925 yrs, $n=10$ ) recruited from the

University of Minnesota participated in the experiment on a voluntary basis. The

participants were all right handed. There were four different conditions that were

separately presented to participants. In each condition, participants were pre

sented with a reference block (6 cm tall). and a randomly chosen comparison

block (heights were between $5.2 \mathrm{~cm}$ and $6.8 \mathrm{~cm}$ tall). Widths of the blocks were

all equal $(9 \mathrm{~cm})$. The participants were seated on a height-adjustable chair in front of the ex

perimental apparatus with their hand resting on a visible start location. The ex

perimental apparatus was made of a rectangle wood board, which acts as a blind,

with one block in the front and one block behind the blind. Participant's viewing 
distance from the front (visual) block was $30 \mathrm{~cm}$.

Participants used only their

right hand to haptically explore the block behind the blind, using only their

thumb and index finger. Participants were asked which of the two blocks was taller and were in

structed to make verbal size judgments in the four following conditions: (1) vi

sion only; participants only vieued the reference and comparison blocks before

making a size judgment. (2) Haptic only; vision was blocked. Participants hapti

cally explored the reference block first, followed by exploring a comparison

block. (3) Visual-to-Haptic ( $\mathrm{V}-\mathrm{H})$; participants first explored the reference block

visually and then haptically explored the comparison block. (4) Haptic-to-Visual

(H-V); participants first explored the reference block haptically and were then

presented with the visual comparison block and subsequent ly indicated which

block was taller. The reference blocks and comparison blocks were randomly

presented for some trials to reduce an order effect. The viewing or haptic explo

ration time was set to 4 seconds and was held constant in all conditions. Each

comparison was repeated three times in each condition. Results and Discussion Four sigmoidal sensitivity functions were created based on each condition.

For each sensitivity function, a just noticeable difference threshold at $75 \%$ cor

rect response level was computed. In addition, we 
determined the region of un

certainty, defined as the region between the $25 \% 75 \%$

correct response level

(i.e., the region where object size was not accurately judged). The main results

are: 1) Vision was more sensitive than haptics for object size discrimination

when only one modality was present (region of uncertainty for vision-only:

$5.866 .06 \mathrm{~cm}$; haptic-only: $5.886 .28 \mathrm{~cm}$; see Fig. la, lb). The region of un

certainty was larger in the haptic-only condition (vision-only: $0.2 \mathrm{~cm}$; haptic

only: $0.4 \mathrm{~cm}$ ). 2) when information from both senses was present, the regions of uncer

tainty increased with respect to both vision-only and haptic-only conditions ( $\mathrm{V}$

$\mathrm{H}: 0.52 \mathrm{~cm} ; \mathrm{H}-\mathrm{V}: 0.47 \mathrm{~cm}$, see Figure 1). 3) For cross-modality comparison conditions, the region of uncertainty was

smaller in $\mathrm{H}-\mathrm{V}$ condition than in the $\mathrm{V}-\mathrm{H}$.

a

$1008>1$

a: :

$\sim 7581$ I $\sim 50^{*}$. J Visual only

$\sim$ I

$\sim 258+-.--$.

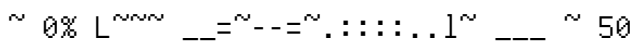

c 55 6.0 6.5 70 STIM SIZE (CM) Visual reference stimulus with haptic comparison slim silt: (CM) b so d ' $00 \%$ Haptic only 556065 STIM SIZE (eM) Haptic reference stimulus witI' visual comparison l:iIMl:iIZI: (CMI 70 
Figure 1. Sigmoidal sensitivity functions for object

discrimination in each of the

four conditions. ' . Dur data confIrm previous [molings

about the saliency of vision for size per

ception. However, we found that the order in which object

size information be

comes available may be important. Allowing for haptic

exploration before view

ing increased discrimination sensitivity. Interestingly, participants tended to

underestimate size in the $\mathrm{HV}$ condition, while

overestimating size in the $\mathrm{V}-\mathrm{H}$

condition. This indicates that the visuo-haptic integration process for discrimi

nating object size is affected by what type of sensory information becomes

available frst (i.e., direction of the sensitivity

function shifts accordingly). In

order to further clarify the relative contribution of each

sense for object size dis

crimination, we are currently investigating size perception acuity during the si

multaneous presentation of haptic and visual cues. It is plausible that the weight

ing of visual and haptic information for object size perception changes as the

presentation order changes. The data of the vision-only condition and haptic-only condition replicated

findings indicating that vision is more sensitive for size discrimination (Ernst \&

Banks, 2002; Gori, Del Viva, Sandini \& Burr, 2008; Helbig \& Ernst, 2008).

However, while the $75 \%$ thresholds were different between vision-only and hap 
tic-only conditions, the lower boundary of the regions of uncertainty $(25 \%$ cor

rect response level) were approximately the same for both vision-only and hap

tic-only conditions. This indicates that it is more difficult to determine differ

ences haptically only when the comparison blocks were larger than the reference

block. There are two possible explanations to why discrimination thresholds were

different in $\mathrm{V}-\mathrm{H}$ versus the $\mathrm{H}-\mathrm{V}$ condition. One explanation, as stated above, is

that the sensitivity of object size discrimination is influenced by how the first

object was perceived (visually or haptically). An alternative explanation for our

data may indicate that judgements during the $\mathrm{H}-\mathrm{V}$ and $\mathrm{V}-\mathrm{H}$ may be influenced

by the distance the blocks were away from the eyes and the body. In our setup,

the physical distance of the visually perceived block was closer than the hapti

cally explored block. That is, the egocentric distances for perceiving the objects

were different for each modality. It could'be possible that this arrangement in

troduced a bias which led to the discrepancy in threshold levels between HV

and $\mathrm{V}-\mathrm{H}$ conditions. A final point of interest is that in the $\mathrm{V}-\mathrm{H}$ condition, the

lower boundary of the region of uncertainty was $6.1 \mathrm{~cm}$, which means that in

758 of the responses, participants incorrectly determined 
smaller than the standard $6.0 \mathrm{~cm}$ block, while $100 \%$ of the participants correctly

determined the $5.9 \mathrm{~cm}$ block to be smaller than the $6.0 \mathrm{~cm}$ standard block, both

being $1 \mathrm{~cm}$ difference in height when compared to the standard block. A new

apparatus is currently being used to control for egocentric distances. This study was designed to provide. baseline data for evaluating clinical

populations with proprioceptive or tactile deficits such as patients with Parkin

son's disease or dystonia. In addition, future studies might explore how the task

requirements (perceptual judgment vs. object-based action) would affect the

Ernst, M. 0., \& Banks, M. S. (2002). Humans integrate visual and haptic information in a statistically optimal fashion. Nature, $415(6870), 429-433$.

Gori, M., Del Viva, M., Sandini, G., \& Burr, D. C. (2008). Young children do not integrate visual and haptic form information. Current Biology : CB, 18(9), 694-698.

Helbig, H. B., \& Ernst, M. 0. (2008). Visual-haptic cue weighting is independent of modality-specific attention. Journal of Vision, $8(1), 21.1-21.16$.

(9) 2009 Taylor \& Francis Group, LLC Perceiving by Dynamic Touch with and without Hands zsolt Palatinus 1 , Claudia Care 1101 , \& Michael T. Turveyl,2

lCenter for the Ecological Study of Perception and Action, University of Connecticut, USA, 2 Haskins Laboratories, New Haven, CT, USA. Non-visual perception of a wielded object's properties is based on the mo

ments of the object's mass distribution. The three moments (mass, static mo

ment and moment of inertia) are invariant over variations in the forces brought 
to bear on an object and on the body's tissues during wielding. Previous research

has shown that perception by dynamic touch is not sensation-based (Carello,

1992), not anatomically specific (Hajnal et al., 2007a), and not dependent on

specific kinematics (Pagano et al., 1993). Importantly, however, it can be selec

tive (Turvey et al., 1996; Cooper et al., 2000). In the present research we extended these investigations beyond the limbs.

The particulars of morphology and physiology-even though they may be

linked to traditional measures of local sensory acuity-should not matter to the

capacity of connective tissue to be structured laufully and globally in support of

dynamic touch (Carello, Silva, Kinsella-Shaw, \& Turvey, 2009). However

wielding is accomplished, a deformation field is induced that is laufully related

to properties of the wielded object. The reported experiments test and compare

the capabilities of the hand and the torso, anatomical links with very different

roles in manipulating objects. Experiment 1 was designed to establish the basic

perceptual capability of the torso, given that it is typically construed more as a

support link than as an effector. Experiment 2 compared torso and hand directly. Method for wielding by torso, rods were attached at their middle to the upper back

with a harness (Figure 1, left). For wielding by hand, rods were grasped in the 
middle. Perceivers (8 per group) used a magnitude production pulley to report

either whole length (markers were moved to indicate both ends of the rod) or

partial length (a single marker was moved to indicate the length of the rod from

the attachment point to the left end). Both experiments used three rod lengths

and three mass conditions. Rods in Experiment 1 were larger $(\mathrm{L}=72$, 96, and

$120 \mathrm{~cm}$; diameter $=2.54 \mathrm{~cm}$; attached mass $=150 \mathrm{~g}$ ) than rods in Experiment 2

$(\mathrm{L}=45,60$ and $75 \mathrm{~cm}$; diameter $=1.25 \mathrm{~cm}$; attached mass = $50 \mathrm{~g})$. The attached 67

mass was affixed at $1 / 8 \mathrm{~L}$ (the rod could be oriented to place the mass on the left

or the right) or the rod remained plain (Figure I, middle). In Experiment $I$, participants received four repetitions of each $L \times M$ com

bination resulting in 36 trials, randomized within blocks. In Experiment 2, they

received three repetitions of the $L \times M$ combinations, randomized within effector

block resulting in 54 total trials. $-\left(1 \mathrm{~A}{ }^{\circ}{ }^{\circ} \mathrm{C}\right.$

Prediction whole ${ }^{\circ}$ a..... - cr. partial A B ". '

.0 c Figure 1 . The style of the attachment (left), the three mass positions (middle), and

the predicted pattern of the judgments (right) . Results and Discussion

Perceived Length, L 033P. A 2 (attention: whole vs. partial length) $\times 3$ (rod

length) $\times 3$ (attached mass position) between-participants ANOV $A$ on $L p 3$ in

Experiment 1 revealed main effects of attention, $F(1,17)$ 
$=16.9, \mathrm{P}<.001$, at

tached mass position, $F(2,17)=18.6, p<.001$, and a Mass $33 \times$ Attention inte

raction, $F(2,17)=57.3, p<.001$. Selective perception by the torso was achieved

in the predicted pattern (Figure 2a).

A 2 (attention) $\times 2$ (effector) $\times 3$ (rod length) $\times 3$

(attached mass position)

between-participant ANOV $A$ on Lp in Experiment 2 revealed main effects of

attention, $F(I, 35)=21.8, p<.001$, mass position, $F(2$, $35)=30.5, p<.001$, and

a Mass $x$ Attention interaction, $F(2,35)=94.7, P<.001$. Neither effector, $F(1$,

$35)=2.42, P=.124$, nor its interactions (8 Fs "'l 1) were significant. The pre

dicted pattern was obtained for both effectors (see Figure 2b) .

Reliability and Accuracy. Reliability of length judgments was assessed by

taking the average deviation of an individual's responses as a 8 of that person's

mean $L p$. A measure of accuracy was provided by mean root square (MRS) er

ror, deviation of an individual's responses as a 8 of actual extent (for details,

see Hajnal et al. 2007a,b). In Experiment $I$, a 2 (attention) $\times 2$ (analysis: reliabil

ity vs. MRS) between participant ANOV $A$ on these measures revealed a main

effect of analysis, $F(1,12)=34.2, p<.001$. MRS is greater than reliability, indi

cating a systematic influence on Lp beyond actual length 
variation). This is the usual finding and is related to the importance of inertial

constraints (see below). There was no interaction between analysis and attention,

< 1. MRS averaged 38.6\%, reliability averaged $14.2 \%$. In Experiment 2 , a 2 (attention) $\times 2$ (grip) $\times 2$ (reliability vs. MRS) be

tween-participant ANOYA again revealed a main effect of analysis, $F(2,35)=$

20.2, $P<.002$. Neither the effect of grip nor its interaction with analysis was

significant, both Fs< 1 . MRS averaged $40.6 \%$ for judgments by hand and

$41.8 \%$ for judgments by torso. Reliability averaged $10.6 \%$ for judgments by

hand and 10.38 for judgments by torso. Inertial scaling. At the outset, we noted that non-visual perception of a

wielded object's properties is based on the moments of the object's mass distri

bution. The difference between MRS and reliability highlights this dependence:

Perceived length is constrained by something other than actual length. Follow

ing common practice (e.g., Cooper et aI., 2000), we conducted regressions of $\log$

perceived length on log Ixx for both experiments. The regression analysis for

perceived whole length revealed a significant dependence on IXx for both torso

and hand, with virtually all of the variance accounted for $(?=.97)$. For per

ceived partial length, a further regression of residuals on Iyz was significant for 
both torso and hand $(?=.92)$. The present results build on findings that the foot is comparable to the

hand in the perception of object length by dynamic touch even in selective tasks

(Hajnal et aI., 2007b). These capabilities are not restricted to the limbs. Tissue

deformation throughout the muscular-skeletal system allous mechanoreceptors

to extract the invariant specific to object extent. More generally, perceivers

seemingly assemble the haptic system into a smart subsystem to pick up the

moments specific to a particular property. It has been suggested, that this func

tion may arise from a synergy of the mechanoreceptors and the attendant neural

and fascia nets (Turvey \& Fonseca, 2009). Dur findings support the hypothesis

that in detecting invariants, the haptic suDstrate behaves in a field-like fashion

suggestive of a tensegrity structure interconnected tension-supporting compo

nents and isolated compression-bearing components that distribute stresses to

ensure force balance and shape stabilization. (a)

Experiment 1 (b) Experiment 2 : : $t^{*}$

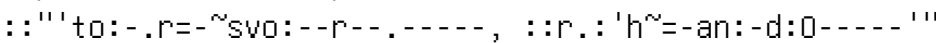

$\mathrm{Lp} / .40 \mathrm{n} 40 \ldots \mathrm{n} 40 \ldots / \ldots 0$ (em) $300 \cdots \cdots \cdot 0$.

$303020200 \ldots \ldots \ldots$. A B C A B * whole length $\theta$ partial length $\mathrm{c} \theta \ldots \ldots . . .0 \cdot 20$ A B c Figure 2.

The expected data pattern for mass conditions $A, B$, and $C$ was obtained in

Carello, C., Fitzpatrick, P., Domaniewicz, 'l., Chan, T.-c., Turvey, M.T. (1992) Effortful touch with minimal movement. Journal of Experimental Psychology: Human Perception and 
Carello, c., Silva, P. L., Kinsella-Shaw, 1. M., \& Turvey, M. T. (2009). Sensory and motor challenges to muscle-based perception. Brazilian Journal of Physical Therapy. 12. 339-350.

Cooper, M., Carello, C., \& Turvey, M. T. (2000). Perceptual independence of whole length, partial length, and hand position in wielding a rod. Journal of Experimental Psychology: Human Perception and Performance, 26,7485 .

Hajnal, A., Fonseca, S. T., Harrison, S., Kinsella-Shaw, 1. M., \& Carello, C. (2007a). Comparison of dynamic (effortful) touch by hand and foot. Journal of Motor Behavior, 39, 82-88.

Hajnal, A., Fonseca, S., Kinsella-Shaw, 1., Silva, P., Carello, C., \& Turvey, M. T. (2007b). Haptic selective attention by foot and by hand. Neuroscience Letters, 419 , $5-9$.

Pagano, C. c., Fitzpatrick, P., \& Turvey, M. T. (1993). Tensorial basis to the constancy of perceived extent over variations of dynamic touch. Perception \& Psychophysics, $54,43-54$.

Runeson, s. (1977). On the possibility of "smart" perceptual mechanisms. Scandinavian Journal of Psychology, $18,172-179$.

Turvey, M. T., \& Fonseca, S. (2009). Nature of motor control: Perspectives and issues. In D. Sternad (Ed.) Progress in motor control: A multidisciplinary perspective (pp. 93-123). New York: Springer Verlag.

van de Langenberg, R., Kingma, I. and Beek, P. 1. (2006). Mechanical invariants are implicated in dynamic touch as a function of their salience in the stimulus flow. Journal of Experimental Psychology: Human Perception and Performance, 32, 1093-1106. Acknowledgments. This research was supported by NSF Grant SBR 00

04097 and a grant from the Provost's office at the University of Connecticut.

(6) 2009 Taylor \& Francis Group, LLC

Apparent Point of Rotatiop. Manipulation Affects 
Multimodal Heaviness Perception Laura Bachus, Kevin Shockley, \& Michael A. Riley University of Cincinnati, Cincinnati, OH USA Heaviness perception is one of the oldest problems in perceptual psychol

ogy (Charpentier, 1891) and yet a unified theory to explain this phenomenon

remains conspicuously absent. Non-visual heaviness

perception (i.e., perception

of heaviness via touchinglhefting an object) has largely been accounted for in

terms of rotational inertia-the resistance of a wielded object to applied wield

ing torque (e.g., Amazeen \& Turvey, 1996; Shockley, Grocki, Carello, \& Tur

vey, 2001). Inertia for rotation in the sagittal plane can be defined as: $1 \sim$ mass. $d \&$ (1)

where $d$ is the distance between the center of mass (eM) and the point of rota

tion (i.e. the urist). Thus, an object's inertia can be manipulated by either chang

ing the mass or the distribution of that mass around the point of rotation. For

example, increasing the distance of a mass on a wielded object auay from the

urist makes the object feel heavier (Amazeen \& Turvey, 1996). A similar

change in inertia can be achieved by changing the object's point of rotation.

When an object is held closer to its center, it will offer less resistance to a given

applied torque than when held at one of its ends. Heaviness perception is also influenced by vision, houever. For example, as

objects become larger, they are typically perceived as lighter, even when con 
trolling for rotational inertia (Amazeen, 19'97). However, theoretical accounts of

size do not explain why size influences appear to depend on seeing the wielded

objects in motion (Masin \& Crestoni, 1981). Streit and colleagues have made

efforts to understand the role of vision in heaviness perception based on our un

derstanding of rotational inertia. They evaluated the influence of changing an

object's apparent motion in response to applied wielding torque based on the

equation: $N=16 . J(2)$

where I is rotational inertia, $w$ is rotational acceleration and $\mathrm{N}$ is applied torque.

They hypothesized that rotational inertia is specified multimodasly by the fol

lowing relation: I N If1Bcifitu!. = "'1Ip.J'1Vn.t (3)

where c.) apparmt is the apparent rotational

acceleration-the acceleration of a

virtual object moving in real time with the actual wielded object (see Figure I)

to which a scaling factor (rotational gain) is applied to manipulate apparent re

sponsiveness of the wielded object to applied torques. Thus, just as an object

that is highly responsive to an applied torque (i.e., low inertia) is perceived as

light, an object that appears to be highly $r^{\circ}$ sponsive specifies to a perceiver that

the object has low inertia and the object is perceived as light (Streit et aI., 2007). At issue is whether the streit et aI. (2007) multimodal inertia model can ac 
count for other visual influences (e.g., object size). One possibility is that the

apparent distribution of mass about a point of rotation may influence heaviness

perception in a similar fashion to actual changes in rotational inertia described

above. For example, if d in Equation I is replaced with dapparen, -the apparent

distance of the center of mass from the point of rotation for a virtual object

then rotational inertia is likewise specified multimodally by the following rela

tion:

Linking Equations 3 and 4 yields . z IlpBcifif1d = mass . dt lpparrnt . $2 \mathrm{~N}$ mass' dapparBnt $=-$ - $^{-}$"'"apI1T $\ldots r$, (4) (5)

which offers a candidate perceptual variable that would capture influences of

both apparent rotational motion and changes in the apparent mass distribution

that would accompany size changes (Amazeen \& Turvey, 1996) - mult imodally

specified mass: (6)

We evaluated a novel prediction from Equation 6. If multimodal heaviness per

ception is sensitive to massspecified, then decreasing the apparent distance of the

center of mass from the point of rotation (dapparent)

should increase the perceived

heaviness of the object. Method In Experiment I $(n=20)$, participants wielded objects while viewing the cor

responding motion of virtual objects in a visual display and reported how heavy 
the objects felt (see Figure 1). Motion sensors on the base of the object handles

tracked the movement of the objects. A computer projected virtual counterparts

of the wielded objects onto a screen in view of the participants. For each trial,

participants wielded the two objects back and forth between two virtual targets

and rated one (test object) relative to the other (standard; heaviness= 1 ob). The

test object was projected in four different apparent point-of-rotation (i.e.

dapparent) configurations and two different values of rotational gain $(0.8$ and $1.2 \times$

actual rotation). The latter manipulation was included to encourage a variety of

responses in case the dapparent manipulation did not influence heaviness. Each

configuration was repeated four times in blocks of eight trials for a total of 32

trials, with trials randomized within each . block for each participant. The ob

jects' motion was recorded during each trial and used to generate identical visual

displays for Experiment 2. During the vision-only condition of Experiment $2(n=20)$, participants

watched the motion recorded from Experiment 1 (without wielding them) and

were asked to report how heavy the test object looked. During the wielding-only

condition, participants were also asked to provide non-visual heaviness judg

ments for the wielded object. Fourvalues of ${ }^{\sim}$ via point of 
ndalon manipulation Figure 1. Illustration of the virtual reality paradigm (left) and the dapparent

manipulation ( $r$ ight). Results and Discussion

Participants' mean heaviness reports were submitted to a 2 (rotational gain)

$x 4$ (dapparent) repeated measures analysis of variance. In Experiment 1 perceived

heaviness was inversely proportional to rotational gain, $F(1,19)=25.52, p<$

.0001 and perceived heaviness was inversely proportional to dapparent, $F(3,19)=$

$12.92, \mathrm{P}<.0001)$, with no interaction. In Experiment 2 there were no significant

effects (Fs < 1). The results supported our hypothesis and demonstrated a novel visual influ

ence on heaviness perception. As the apparent distance between the center of

mass and the point of rotation increases, perceived heaviness decreases. Percep

tual sensitivity to the hypothesized quant $i^{\sim}$ massspecified may, therefore, account 73

for previously observed visual influences of both rotational acceleration (e.g.,

Streit et aI., 2007) and size (e.g., Ellis \& Lederman, 1998) on heaviness percep

tion under a common theoretical framework based on rotational inertia. For ex

ample, when objects increase in length, the center of mass extends farther from

the point of rotation and yields a decrease in perceived heaviness. However,

influences of apparent size and rotational gain only occurred during multimodal

(e.g. visual + haptic) perception, suggesting that these 
heaviness perception by virtue of their relation to applied wielding torques. Expeh m ent 1: Multimo dal Perception 01180 gj $160 .{ }^{\circ} 140 \sim 120$ I100 "2 80 2: 60 0.J $\sim 40 \sim 20$ Rotati onal G a in -0.0 .8 .1 .2$ $0^{\sim}-\ldots-\ldots(J) 180 \sim 160$ C . $5140 \sim 120$ I100 $\sim 80 . \approx 60$ G. $\sim 40$ G.I 200.7 .512 .517 .5 22.5 Distance of Apparent Ce nter of Mass from Wtist (cm) Expe rim ent '2: Visual Perception Rotational Gain - $0 \cdot 0.8$

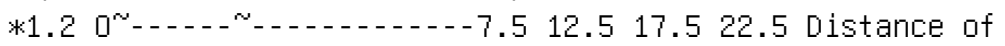
Apparent Center of Mass from wtist (cm)

Amazeen, E. (1997). The effects of volume on perceived heaviness by dynamic touch: with and without vision. Ecological Psychology, 9, 245-263.

Amazeen, E., \& Turvey, M.T. (1996). Weight perception and the haptic sizeweight illusion are functions of the inertia tensor. Journal of Experimental Psychology: Human Perception and Performance, 22, 213-232.

Chapentier, A. (1891). Analyse experimentale de quelques elements de la sensation de poids [Experimental study of some aspects of weight perception]. Archives de PhysiologieNormales et Pathologiques, 13, 122-135.

Ellis, R. R. \& Lederman, S. 1. (1998). The golf-ball illusion: evidence for topdoun processing in weight perception. Perception, 27, 193-201.

Garg, A., \& Saxena, U. (1980). Container characteristics and maximum acceptable weight of lift. Human Factors, 22 , 487-495.

Masin, C. S. \& Crestoni, L. (1981). Experimental demonstration of the sensory basis of the size-ueight illusion. Perception \& Psychophysics, 44, 309312.

Streit, M., Shockley, K., \& Riley, M. (2007). Rotational inertia and multimodal heaviness perception. Psychonomic Bulletin \& Review, 14, 1001-1006.

Acknowledgements. This research was supported by NSF grant H0716319.

(c) 2009 Taylor \& Francis Group, LLC Twelve-Month-0lds' Discrimination of Monkey Faces: Evidence for Perceptual Narrouing? 
Ross Flom, Melissa Wright, Amanda Phillippi, Tom Beckstead, Jacob Jones, Harrison Allen, \& Danny Boysen Department of Psychology Brigham Young University It is well known from birth that faces are salient, preferred over other

stimuli (Easterbrook, Kisilevsky, Hains \& Muir, 1999;

Barrera \& Mauer, 1981),

and are readily discriminated (Mauer \& Young, 1983;

Pascalis \& de Schonen,

1994). It has also been shown that over the course of development infants'

proclivity for face discrimination is influenced by their social environment.

Pascalis, de Hann, and Nelson (2002), for example, demonstrate that 6 -month

olds are able to discriminate various monkey faces as well as human faces

whereas 9-month-olds can only discriminate different human faces. Importantly,

however, if 6-month-olds receive $1-2$ minutes per day of familiarization with

photographs of monkey faces three months later the now 9-month-olds can still

discriminate unfamiliar and familiar monkey faces

(Pascalis, Scott, Kelly,

Shannon, Nicholson, Coleman, \& Nelson, 2005). Like face perception, infants' discrimination of speech also varies as a

function of experience and exposure. Specifically, 4to 6-month-olds

discriminate speech sounds in their native and non-native languages; however,

by 10to 12 -months of age infants can only discriminate speech sounds

common to their native language (Kuhl, Williams, Lacerda, Stevens, \& 
Lindblom, 1992; Werker \& Tees, 1984). More recently,

houever, Kuhl, Tsao,

and Liu (2003) have shown that providing 9-month-olds with exposure to non

native phonemes extends their discrimination of these phonemes to 12 -months

of age. The fact that within the first months of life infants' discriminate a variety of

faces and speech sounds and by infants first birthday this ability has "narrowed"

to reflect infants' perceptual experience has become knoun as "perceptual

narrouing" (Pascalis, et aI., 2002). Critical, houever, is the assumption that

perceptual narrowing reflects a relatively permanent change in perceptual

abilities as well as a change in early neural architecture (Scott, Pascalis, \&

Nelson, 2008). The purpose of this experiment is to examine whether infants'

ability to discriminate unfamiliar monkey faces truly narrous. One possibility,

and the possibility examined in this experiment, is that 12-month-olds who have

never been previously exposed to monkey faces, can discriminate unfamiliar

monkey faces when they are provided longer periods of familiarization and

longer times to visually compare the two faces. In this experiment, we examined

12-month-olds' discrimination of monkey faces when provided $40 \mathrm{~s}$ or $20 \mathrm{~s}$ of

familiarization and were subsequently allowed to compare 
or 5s. Method Forty-eight 12-month-olds were familiarized to a static color display of a

Barbary Macaque (Macaca sylvanus). The faces used by Pascalis et. al. (2002)

were used in the current experiment. Figure 1 provides an example of the stimu

li. Infants were randomly assigned to one of two familiarization conditions (40s

or 20s). During familiarization infants saw the same face side-by-side on two

video monitors. Following familiarization infants received two test trials where

the face of familiarization was presented on one video monitor and a novel face

was presented on the adjacent monitor. For those infants who received $40 s$ of

familiarization each of the two visual-paired comparison test trial were los in

length. Those infants who received $20 s$ of familiarization each test trials was $5 \mathrm{~s}$

in length. The lateral position of the novel face was alternated between the two

test trials. Following the two test trials the procedure was repeated where each

infant received a different pair of faces. Figure 1: Examples of stimuli : Each infant received two pairs. 77 Results and Discussion The primary dependent variable was infants' proportion of total looking

time (PTL T) directed touard the novel face. Proportions were derived by

dividing the time infants spent looking to the novel face by the time spent

looking at both faces. An overall PTL T was derived by 
averaging across the four

test trials. Infants' proportion of total looking time (PTL

T) to the novel face is

presented in Figure 2. Following 20 s of familiarization and the two $5 \mathrm{~s}$ visual

paired comparison test trials 12 -month-olds failed to show a reliable preference

for the novel or familiar face $M=498 ; t(23)=.98, p>$ .1 within the $20 \mathrm{~s}$

familiarization condition 12-month-olds' looking behavior between the first

familiarization and test-trial pairing and the second pairing did not significantly

differ ( $p$. I). Following 40 s of familiarization and two los test trials 12 -month

olds, houever, shoued reliable discrimination of the novel and familiar monkey

face, $M=548 ; t(23)=3.1, P=.015$. In addition, 12 -month-olds showed

reliable discrimination for the first pair, $M=55 \%$; $(23)=2.8, P=.02$, as well

as the second, $M=53 \% ; t(23)=2.6, P^{\sim} .02$. Finally, infants looking to the

novel face was significantly longer in the 40 s familiarization condition

compared to the 20 s condition $t(23)=4.1, P<.01$. Thus 12-month-olds show

reliable discrimination of previously unfamiliar monkey faces when provided

longer familiarization and increased time to visually compare the two faces. 0.75.49 (.09) .47 .54*(.06) $.55 *(.10) \sim 0.5$

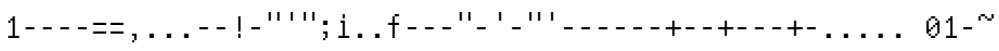
$: l^{\prime}$ 


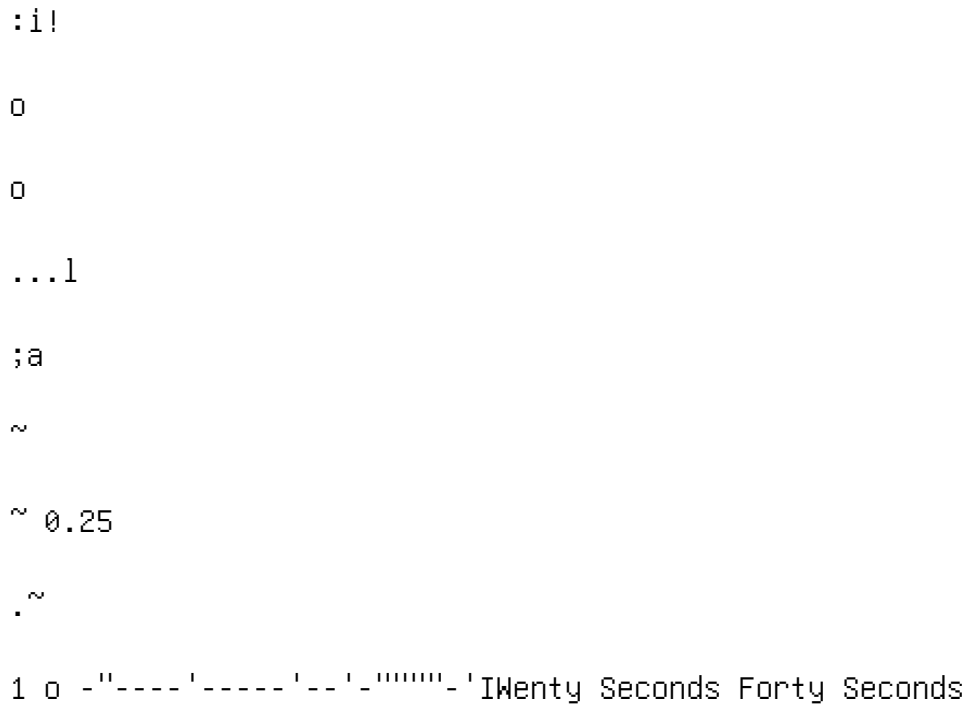
Amount of Familiarization a Pairs 1 \& 2 Combined Pair 1 (trials 1.2) - Pair 2 (trials 34) . .5=ChaIlu " p<.05 Figure 2: Mean (So) proportion of total looking time (PTL T) to the novel face.

These results demonstrate that infants' ability to discriminate unfamiliar

monkey faces is not "lost" or "perceptually narroued" during the course of

development. Specifically, our results demonstrate that 12-month-olds who have

limited exposure and time to compare the two faces do not show significant

evidence of discriminating the novel and familiar face. However, those infants

who received longer periods of familiarization and comparison reliably

discriminated the faces. Finally, it is someuhat ironic that studies of perceptual

narrowing have been used to highlight the experience dependent nature of

perceptual learning where the current results, that do not show a perceptual 
Barrera, M., \& Maurer, D. (1981). Recognition of mother's photographed face by the three month old infant. Child Development, $52,714-716$.

Easterbrook, M., Kisilevsky, B., Hains, S., \& Muir, D. (1999). Faceness or complexity: evidence from neuborn. visual tracking of face like stimuli. Infant Behavior and Development, $22,17-35$.

Kuhl, P. K., Tsao. F.-M., \& Liu, H.-M. (2003).

Foreign-language experience in infancy: Effects of short-term exposure and social interaction on phonetic learning. Proceedings of the National Academy of Sciences, USA, $100,9096-9101$.

Kuh1, P. K., Williams, K. A., Lacerda, F., Stevens, K. N., \& Lindblom, B. (1992). Linguistic experience alters phonetic perception in infants by 6 months of age. Science, 255, 606-608.

Maurer, D., \& Young, R.E. (1983). Neuborns' following of natural and distorted arrangements of facial features. Infant Behavior and Development, 6, 127-131.

Pascalis, 0., de Hann, M., \& Nelson, C.A. (2002). Is face processing speciesspecific during the first year oflife? Science, 296, 1321-1323

Pascalis, 0., \& de Schonen, S. (1994). Recognition in 3to 4-day-old human neonates. NeuroReport, 5, 1721-1724.

Pascalis, ø., Scott, L.S., Kelly, D. J., Shannon, R.W., Nicholson, E., Coleman, M., \& Nelson, C.A. (2005).

Plasticity of face processing in infancy. Proceedings of the National Academy of Sciences, USA, 102,5297-5300.

Scott, L.S., Pascalis, 0., \& Nelson, C.A. (2007) A domain general theory of the development of perceptual discrimination. Current Directions in Psychological Science, $16(4), 197-201$

Werker, J. F., \& Tees, R.C. (1984). Cross-language speech perception: Evidence for perceptual reorganization during the first year of life. Infant Behavior and Development, $7,49-63$.

(9) 2009 Taylor \& Francis Group, LLC Human Sensitivity to Local Taus Nam-Gyoon Kim 1 \& Keonho Shine IDepartment of Psychology, Keimyung University, Daegu, Korea zDepartment of Education, Kangnam University, Yongin, Korea when an 
object moves directly toward an observer, its arrival time can be

predicted from tuo types oflocal perturbation in the optic flow. The visual angle

subtended by any two texture elements on the surface of the object expands as it

approaches the observer, as does the image corresponding to the entire contour

of the object. The inverse of the relative rates of each of these tuo types of ex

pansion defines the time to contact (TTC). Tresilian (1991) refers to these two

optical quantities as local taul cone-dimensional property) and local tau2 (two

dimensional property), respectively. For these quantities to be available, the contour of the object must remain

constant over the course of its approach. If the object is non-spherical, such as a

rugby ball or an American football, that rotates on its way to the receiver, not

only does its shape deform irregularly, but the surface texture elements move

about, sometimes even disappearing from view. How might the visual system

cope under such circumstances? Gray and Regan (2000)

studied the estimation of TTC for a rotating non

spherical object. In that study, an oval object rotated about its horizontal axis

over the simulated path of approach so that the distance between the two end

points along the vertical axis of the object changed, altering the outline of the

object from ellipse to circle or circle to ellipse. Local

tau 2, therefore, failed to 
convey veridical TTC information. However, the distance between the two end

points along the horizontal axis remained constant, which could have been used

to extract local tau!. Gray and Regan observed that TTC estimation was unreli

able under monocular viewing, but improved under binocular viewing. Are human observers incapable of estimating TTC of a tumbling rugby ball

while watching it with a single eye, as Gray and Regan (2000) contend? Every

day experiences suggest otherwise. In Gray and Regan's study, the oval object

rotated only 90 deg so that, for a given trial, its projected shape changed either

from circle to ellipse or from ellipse to circle depending on the initial orientation

of the object. Thus, despite the fact that an infinite variety of optical patterns can

be engendered by rotating non-spherical objects, only two types of deformation

were depicted in Gray and Regan's study. For that reason, additional studies are

clearly warranted. Here we report the results of two experiments directed at estimating TTC of

rotating non-spherical objects. In the first experiment, textureless, non-spherical

objects rotated either about one or two axe's. In the former, the distance between

the two end points along the axis of rotation could be used to defme local tauz;

in the latter, no such optical pattern was available, compromising local tauz. In 
the second experiment, the density of object's surface

texture varied, compro

mising local tau 1. Experiment 1: Local Tau2 Method Fourteen experimentally naIve participants, all graduates (4) males and 10

females) from Keimyung University, volunteered for the experiment. Displays were presented on a $106.7 \mathrm{~cm}(42$ in.) LCD monitor subtending a

fIeld of view of $63.7^{\circ} \mathrm{H} \times 38.9^{\circ} \mathrm{V}$ when viewed at a distance of $75 \mathrm{~cm}$. Displays

simulated a projectile approaching the observer along the sagittal plane parallel

to the ground plane. The object was an ellipsoid (either a disk-shaped object or a

rugby ball-shaped object) with its three semi-axes of different lengths. Five variables were controlled. The values of TTC varied among $1.8,3.0$,

4.2 , and $5.4 \mathrm{~s}$ and were further jittered within $\pm 0.2 \mathrm{~s}$ to minimize response bias.

The four TTC values were combined with two approach velocities of 6,0 and 9.0

mls to determine the starting location of each object. The duration of the ap

proach also varied within a range of $2.5 \pm 0.25 \mathrm{~s}$. The object rotated about one

axis (either the vertical or horizontal axis) or about two axes, and completed a

quarter tum (90 deg), a half tum (180 deg), or a full tum (360 deg), during its

approach. The combined effects of rotation (i.e., axis and amount of rotation) were

various patterns of deformation on the non-spherical objects but negligible de

formation on the spherical (control) objects, which were included because they 
can engender reliable local tauz. The spherical objects, therefore, were con

trolled by TTC and velocity only, with each combination repeating three times

for a total of 24 trials. These manipulations yielded a 2 (Velocity: 6 or $9 \mathrm{mls}$ ) $\times 4$

(TTC: $1.8,3.0,4.2$, or $5.4 \mathrm{~s}) \times 2$ (0bject Type: Disk or Rugby ball) $\times 3$ (Amount

of Rotation: quarter, half, or full tum) $\times 3$ (Axis of Rotation: Vertical, Horizon

tal, or Vertical + Horizontal) design with a total of 144 trials with non-spherical

objects plus 24 trials with spherical objects, All variables were controlled

within-subjects. Trials were initiated when the participant pressed the space bar to trigger the

display. On termination of the display, participants were asked to predict when

the object would reach them had it kept coming at the same speed and to press a

key on the keyboard at the moment that coincided with their predicted time of

the object's arrival. Feedback uas provided throughout the experiment. 81 Results and Discussion Best fit lines were computed by regressing perceived against actual TTC for

each object type. The analyses yielded regression equations: $y=0.52 x+1.27$

for disk, $y=0.59 x+1.39$ for rugby ball, and $y=0.65 x$ +0.85 for sphere.

Slopes of 0.52 and 0.59 in the non-spherical object conditions were comparable

to those reported in similar TTC studies (e.g., 0.57 for inexperienced drivers 
reported by Cavallo \& Laurent, $1988 ; 0.61$ by Schiff \& Detwiler, 1979). Correlations were obtained between actual TTC and perceived TTC in each

condition of rotation axis for each participant. The results were entered into an

analysis of variance (ANOY $A$ ). The group effect was not significant, $F(2,26)<$

I. These results demonstrated that human observers can perceive the TTC of

rotating non-spherical objects, although less reliably, than that of spherical ob

jects. Feedback may have facilitated participants' TTC judgments. Nevertheless,

the results are remarkable considering the fact that local tau2, a possible source

of TTC information in this experiment, was severely compromised with non

systematically deforming contours. Experiment 2: Local Taut Method Fourteen undergraduates and graduates (5 males and 9 females) from Kei

myung University participated in the experiment for a nominal fee. The same apparatus, viewing geometry, and procedure used in Experiment

1 were used. objects were depicted with random dots whose density varied

among 4, 16, 64, and 256. Because these objects were volumetric, roughly half

of the surface dots were projected to the observation point. Five variables were controlled. The values of TTC varied among $1.8,3.6$,

and $5.4 \mathrm{~s}$ and were further jittered within $\pm 0.2 \mathrm{~s}$. Approach velocity varied

within a range of $7.5 \pm 1.0 \mathrm{mfs}$; the duration of the approach varied within a 
range of $2.5 \pm 0.25 \mathrm{~s}$. As in Experiment 1 , objects rotated in three different

ways, that is, about one axis (either the vertical or horizontal axis) or about two

axes, and underwent either a half turn (180 deg) or a full turn (360 deg). These

manipulations yielded a 4 (Dot Density) $\times 3$ (TTC) $\times 3$

(object) $\times 2$ (Amount of

Rotation) $\times 3$ (Axis of Rotation) design for a total of 216 completely random

ized trials. All variables were controlled within-subjects. Results and Discussion Performance in each condition of dot density can be described by regression

equations: $y=0.72 x+0.71$ for the 256 dot condition, $y$ $=0.65 x+0.93$ for the

64 dot condition, $y=0.58 x+1.38$ for the 16 dot condition, and $y=0.44 x+$

2.50 for the 4 dot condition, respectively. The slope for the 4 dot condition was

substantially lower than those of the other three higher density conditions. For further analysis, correlations were obtained between actual TTC and

perceived TTC in each condition of dot density for each participant. The results

were entered into an ANov $A$. The effect of dot density was significant, $F(3,39)$

$=24.44, \mathrm{P}<.0001$. The mean correlation for the 4 dot condition $(M=.49)$ dif

fered from the means of the three higher density conditions $(M=.77$ for 256

dots, $M=.71$ for 64 dots, and $M=.66$ for 16 dots). Despite the degraded per

formance in the 4 dot condition, the correlation coefficients of the 14 partici 
pants all reached significance. These results indicate that participants were still

capable of estimating TTC, albeit to a limited degree. Note that in the 4 dot con

dition, only about 2 dots were projected to the observation point and these were

displaced or even disappeared and were replaced by dots hidden behind due to

rotation. It appears, therefore, that the visual system responds to even the slight

est indication of optical expansion to extract requisite information, such as local

tau 1. General Discussion Two experiments were directed at the perceptual capacity to estimate TTC

of an approaching object even under severely perturbed or impover ished optic

flow, condition intended to compromise sources of TTC information, in particu

lar, local taul and local tau2. The results demonstrated that human observers are

capable of estimating TTC even under severely compromised conditions, re

sponding to even the slightest indication of optical expansion to estimate TTC of

Cavallo, V., \& Laurent, M. (1988). Visual information and skill level in time-tocollision estimation. Perception, $17,623-632$.

Gray, R., \& Regan, D. (2000). Estimating time to collision with a rotating nonspherical object. Vision Research, $40,49-63$.

Schiff, W., \& Detwiler, M. L. (1979). Information used in judging impending collision. Perception, 8, 647-58.

Tresilian, J. R. (1991). Empirical and theoretical issues in the perception of time to contact. Journal of

Experimental Psychology: Human Perception and Performance, 
17, 865-876. Acknowledgements. This research is supported by a Korea Research Foun

dation grant (KRF-2008-327-H00020) awarded to Nam-Gyoon Kim.

(6) 2009 Taylor \& Francis Group, LLC

Analyses of User's Action for Perceiving Shapes Using an Active Perception Device Ryo Mizuno!, Kigohide !to 2,8 Makoto okamoto 2

1 Graduate school of System Information Science, Future University-Hakodate, JAPAN 2 School of System Information Science, Future University-Hakodate, JAPAN In recent years, there has been great progress in the development of sensor

technology for assisting persons in the perception of their environment. Such

devices obtain data and transmit it to user, and this data must be provided as

meaningful information to the user. Therefore, such sensing device should be

designed with human perceptual capability in mind. Humans pick up informa

tion from their environment by employing perceptual systems (Reed \& Jones,

1982). Therefore, if a sensing device is to aid the perception of the user, should

also be able to move in a way that mimics perceptual systems. Mizuno, Ito,

Akita, 0no, Komatsu \& okamoto (2008) called such moveable sensing devices

"Active Perception Devices". Palmsonar is one such active perception device. It is used specifically to aid

perception of environmental properties in the visually impaired. Palmsonar

measures the distance between the user and an object by an ultrasonic sensor 
and transmits this information through vibrations applied to the user's palm.

Palmsonar not only measures and transmits the distance between a user and an

object, but also aids in perception of the shapes of the objects without vision.

We conducted two experiments that compared how expert and novice users use

the Palmsonar in a shape perception task. Experiment 1 Experiment 1 investigated the ability of novice participants to use the Palm

sonar to perceive shape. Method Participants were nine university students. They had never used Palmsonar

prior to the experiment. They were blindfolded.

Participants used the Palmsonar

to explore four shapes-"sphere", "cube", "rectangular solid", and "cylinder"

while maintaining a $1.5 \mathrm{~m}$ radius from each shape. objects surfaces were con

structed from paper. The volume of each object was 47,713 cm 3 . The task of the participant was to report the perceived shape of each object.

objects were presented randomly. There were 32 trials consisting of eight tri

als for each of the four objects. Before the experimental trials, there were

training trials. In training trials, there were two shapes: "cone" and "triangle

pole". Trials lasted 2 minutes. Results and Discussion

The mean percentage of correct identification for the sphere, cube, rectangu

lar solid, and cylinder were 27.78, 51.94, 30.56, 26.39, respectively. A percen

tage response matrix for this experiment can be seen in 
Table 1. T-tests com

pared the mean percentage of correct identification for each shape relative to the

chance level. Results of the T -test shous tp.at performance with cube was better

than chance, $t(16)=2.80, P<.05$ level. By contrast, performance with sphere,

rectangular solid and cylinder was not significantly better than chance level.

These results indicate that shape identification is quite difficult for novice per

ceivers, and shapes with sharp edges are more easily identified than shapes with

smooth edges. Table 1 . Mean percentage of responses for each shape SPHERE SPHERE 27.78 CUBE ILl 1 RECTANGIJLAR

11.11 CYLINDER 50.00 Experiment 2 CUBE 31.5451 .94

12.504 .17 RECTANGULAR CYLINDER $22.22 \quad 37.5030 .56$

9.7244 .4419 .449 .7226 .39 The purpose of this experiment is to investigate difference in the move

ments used by experts and novices in perceiving shape using Palmsonar. Method The participants were an expert and a novice at shape identification using

Palmsonar. The expert had trained shape identification for 3 days (2 hours/day).

In training trials, there were two shapes: "cone" and "triangle pole". The nov

ice participant had never used Palmsonar prior to this experiment. The expert

and the novice were university students. Materials and procedure were the same

as experiment 1 . In this experiment, the user's movements were captured by two

video cameras and motion capture system (PV Studio 30). The 16 trials con

sisted of four trials for each of the four objects. 85 
Results and Discussion The percentage of correct identification by expert and novice were 87.5 and

31.3 respectively. Analysis of movement data focused on a) The regularity of

sensor's motion at vertical direction, b) changes in amplitude in time series, c)

differences in amplitude among each body part. Figure 1 shous wave patterns of

expert and novice at horizontal direction. First, the auto correlation coefficient . was calculated in order to verify the

regularity of sensor's motion at vertical direction. Figure 2 presents the auto

correlation function of series of sensor's position at vertical direction. The mean

of square values of auto correlation efficient (r2) were compared in aT-test. The

auto correlation efficient at highest point of expert's motion is higher than no

vice's motion significantly, $t(15)=4.54, P<.01$. This suggests that regularity of

motion at vertical direction is effective for shape identification. Next, in order to verify changes of amplitude in time series, time series

were divided three phases, and standard deviation (SD) of sensor position at

horizontal direction were calculated in each phase (see Figure 2). In the case of

novice, $S 0$ increased over time $\left(1.29,1.28^{\prime}, 1.57\right)$. To the contrary, in the case of

expert, SD decreased over time $(1.39,0.92,0.79)$. T-tests compared the mean

standard deviation for each phase of expert with novice's. In the first phase, 
there was no difference between them. At middle phase and

final phase, the $\mathrm{SD}$

of the expert's was smaller than that of the novice (Middle phase: $t(26)=-2.35$,

$P<.02$, Final phase: $t(26)=-3.96, P<.001)$. This

result indicates that experts

move the sensor slowly decrease the variability with which they move the sensor

over time. In other words, effective search of information for shape perception

using an active sensing device is to change moving sensor from rough search to

detail search. Finally, the mean SD of each position of body parts and sensor at horizontal

direction were calculated. In the case of expert, $\mathrm{SD}$ of the sensor, urist, elbou,

and shoulder were $1.63,1.64,1.71$, and 1.83 respectively. In the case of novice,

SD of the sensor, wrist, elbow, and shoulder were 2.06, $1.9,1.57$, and $1.49 \mathrm{re}$

spectively (see Figure 3). In general, the movements of the novice had greater

variability than those of the expert.

$100-100^{\circ}$

$100 r \ldots$

5100.

100

100L.-................ 01020304050607080 90100110120 Time (sec)

$100-100^{\circ}$

$\sim, 00 \cdots 1$ 
Mizuno, R., Ito, K., Akita, 1., Ono, T., .Komatsu, T., \& okamoto, M. (2008). Shape Perception using CyARM Active Sensing Device. International Conference of Cognitive Science 2008, 182-185.

Reed, E., \& Jones, R. (1982). Reasons for Realism. Laurence Erlbaum Associates.

(9) 2009 Taylor \& Francis Group, LLC Improvements in Perceptual Accuracy Scale to Frequency of Feedback about Task Performance Jeffrey B. Wagman \& Daun M. McBride Department of Psychology, Illinois State University Perceptualleaming is the improvement of perceptual skill with practice (E.

Gibson, 1969). In general, perceptual consistency improves with repeated per

ceptual experience regardless of whether that experience includes any informa

tion about task performance (Wagman et aI., 2001). However, perceptual accu

racy improves only when such experience includes information about how per

ceived values compare to actual values (withagen 8 Michaels, 2005). A recent study by Wagman et aI. (2008) compared post-test improvements

in perceptual accuracy when perceivers were provided with feedback about task

performance (knowledge of results, or ${ }^{*} R$ ) on different proportions of trials

during a practice session. In this study, participants reported the perceived

length of wielded occluded $T$-shaped objects in a pre-test, practice session, and

post-test. This study resulted in three important findings. First, consistent with

previous research, there were post-test improvements in perceptual accuracy 
(defined in terms of changes in slope and intercept of the regression lines relat

ing perceived length to actual length) only when KR was provided during prac:

tice trials. Second, such improvements occurred even when KR was only pro

vided on a portion of practice trials. In particular, post-test perceptual accuracy

improved when KR was presented on 1008 of practice trials and when $\mathrm{KR}$ was

provided on $50 \%$ of practice trials but not when $K R$ was presented on $25 \%$ or

12.5\% of practice trials. Third, there was no difference in post-test improve

ments when KR was presented on 1008 of practice trials and when $K R$ was

provided on $50 \%$ of practice trials. In short, the results suggest that in this task, (a) providing $\mathrm{KR}$ on $50 \%$ of

practice trials was as effective in bringing about improvements in perceptual

accuracy as providing $K R$ on $100 \%$ of practice trials and (b) providing $\mathrm{KR}$ on

258 of practice trials was as ineffective in bringing about such improvements as

providing $K R$ on $\theta 8$ of practice trials. In other words, the results seem to estab

lish endpoints at which the embedding of $\mathrm{KR}$ during practice is effective at im

proving post-test perceptual accuracy (and at which the embedding of $\mathrm{KR}$ is

ineffective at doing so). The current experiment is an attempt to further these 89

findings by investigating such improvements for the lack 
bedding ofKR in a practice session falls between these endpoints. Method one hundred four (104) students from Illinois State University participated

in this experiment. They were randomly assigned to one of seven $K R$ conditions

$(.50, .46, .42, .38, .33, .29$, and $.25 K R)$. The number preceding the KR refers to

the proportion of $\mathrm{KR}$ trials provided in the practice session. The experiment

consisted of a pre-test, practice session, and post-test. In the pre-test, participants wielded occluded T-shaped objects and reported

the perceived length of each object by me-ans of magnitude production (i.e., by

adjusting a moveable flag to correspond to the perceived length of the object).

six objects were wielded four times each, and the order of presentation of ob

jects was randomized. In the practice session, participants performed the same

task using a different set of six $T$ shaped objects. The practice session consisted

of two different types of trials-KR and No-KR. No-KR trials proceeded as in

the pre-test. KR-trials proceeded in the same way except that after a participant

provided their perceptual report, the experimenter readjusted the distance of the

flag to correspond to the actual length of the object. The number of KR-trials in

the practice session depended on the experimental condition. There uere tuelve

KR-trials in the $0.50 \mathrm{KR}$ condition, eleven in the $0.46 \mathrm{KR}$ 
condition, ten in the

$0.42 K R$ condition, nine in the $0.38 K R$ condition, eight in the . $33 K R$ condition,

seven in the .29kR condition, and six in the .25KR

condition. KR-trials were

distributed as evenly as possible across the practice session. The post-test pro

ceeded exactly as the pre-test. Results and Discussion For each participant, we compl!ted regression lines with perceived

length as the dependent variable and actual length as the independent variable in

both the pre-test and post-test. We then calculated

difference scores for the slope

and intercept of the regression lines for each

participant. Given that we expected

slopes to increase from pre-test to post-test (slopes in the pre-test tended to be

less than 1.0), difference scores for slope were calculated by subtracting pre-test

values from post-test values. Given that we expected intercept to decrease from

pre-test to post-test (intercepts in the pre-test tended to be greater than 0.0), dif

ferences scores for intercept were calculated by subtracting post-test values from

pre-test values. A one-sample t-test found that difference scores for slope $(M=.24)$ were

greater than $0[t(103)=11.37, \mathrm{P}<.00$ I] (i.e., slopes tended to increase from pre

test to post-test). However, a one-uay ANov A found no differences among

difference scores for slope across the seven $K R$ conditions $[F(6,97)=1.60, P=$ 
.16]. Similarly, a one-sample t-test found that difference scores for intercept (M

= 8.1) were also greater than $\theta[t(103)=9.43, P<.001]$

(i.e. intercepts tended to

decrease from pre-test to post-test). However, a one-way ANDV $A$ found no

differences among difference scores for intercept across the seven $K R$ condi

tions $[F<1]$. In summary, the results suggest that perceptual accuracy improved

from pre-test to post-test and that the degree of improvement did not differ

across KR conditions. The findings that (a) the degree of improvement in perceptual accuracy did

not vary across KR conditions and (b) post-test perceptual accuracy improved

when KR was provided on $25 \%$ of practice trials are someuhat contrary to those

of Wagman et al. (2008). As a result, we made an attempt to explicitly compare

the results of the two studies. We calculated difference scores for the slopes and

intercepts reported by Wagman et al. (in the same manner as in the current expe

riment) and created learning curves (for slope and intercept, respectively) using

the difference scores from both experiments (see Figures 1 and 2). e $10=0$. ${ }^{\sim}$ a. . sa $\mathrm{rn}<1 \quad 0.0 .25$ - D. Data from Waeman et aI. (2088) - Data from enrrent experiment .so .75 1.0 KR Condition Figure 1. A power law function accounted for $69 \%$ of the variance in slope differ

ence scores when the data from the current experiment were combined with the data from 
Wagman et al. (2008). In each case, a power law function provided a better fit of the resultant

learning curve than an exponential function. The power law function accounted

for 698 of the variance in slope difference scores and $63 \%$ of the variance in

intercept difference scores (see Figure 1 and Figure 2). such findings suggest

that across experiments, perceptual accuracy improves at a rate that is mathe

matically scaled to the frequency with which the perceiver is provided with in

formation about task performance and suggests that like many other cognitive,

motor, and perceptual skills, perceptual learning exhibits pouer law structure

(see J. Anderson, 2000, for a review). $1 Z^{\prime} \sim \theta^{\infty} \mathrm{I}$

$\sim 1 \ldots \sim^{\prime}-1$ Q.,${ }^{\infty} \ldots{ }^{\infty}=\ldots<<10.25$

- D - D Data from Wagman et at. (2008) - Data from current experiment .50 .75 1.0 KR Condition 91 Figure 2. A power law function accounted for 638 of the variance in intercept dif

ference scores when the data from the current experiment were combined with the data

Anderson, J. R. (2000). Learning and memory. New York: wiley.

Gibson, E. 1. (1969). Principles of perceptual learning and development. New York: Appleton.

Wagman, 1. B., Shockley, K., Riley, M. A., \& Turvey, M. T. (2001) Attunement, calibration, and exploration in fast haptic perceptual learning. Journal of Motor Behavior, 33(4) 323-327. .

Wagman, J. B., McBride, D. M., \& Trefzger, A. J. (2008). Perceptual experience and post-test improvements in perceptual accuracy. Perception \& Psychophysics, 70 , $1060-1067$. 
Withagen, R. \& Michaels, C. F. (2005). The role offeedback information for calibration and attunement in perceiving length by dynamic touch. Journal of Experimental

Psychology: Human Perception \& Performance. 31, 13791390. Acknowledgements. We thank Jeremy orr and Alex Gomory for help with

data collection

(c) 2009 Taylor \& Francis Group, LLC The Influence of Eyeheight and optic flow on Egocentric Distance Perception Jonathan Shook, Eliah white, \& Kevin Shockley University of Cincinnati, Cincinnati, OH USA Perception of extent in the environment has been shown to be a function of

the effective eyeheight of an observer-the height from the line of sight to the

ground. Ooi and He (2007) proposed that distances may be coded in terms of the

angle subtended between the extent on the ground scaled to one's eyeheight. (cf.

Mark, 1987; Warren \& Whang, 1987; Wraga \& Proffitt, 2000 , for similar scal

ing of environmental layout in other spatial dimensions). In other words, if one

is given a target distance (i.e., extent) to be reported, one will report in terms of

the angle subtended which corresponds to different extents as eyeheight

changes. Perception of distance, however, has .also been shown to be a function of

optic flow. Changes in optic flow velocity (i.e. the rate of flow of texture ele

ments in one's field of view) while walking on a treadmill have been shown to

affect egocentric distance estimations (i.e., perceived self-motion) (e.g., Proffitt

2003). This general phenomenon has been attributed to calibration between vis 
ual and kinesthetic information about forward motion (e.g., Rieser, Pick, Ash

mead, \& Garing, 1995). At issue is the visual information about forward motion

that is scaled to kinesthetic information about foruard motion. One possibility is

optic flow velocity (a global measure of the rate of optic flow in one's field of

view). However, all of the texture elements in an observer's field of view do not

transform in a uniform fashion. Elements uhich are closer to the observer flow

by the observer at a faster rate than elements farther away. Imagine an observer

walking down a hallway. Texture elements on the ground will flow by faster for

a short observer than for a tall observer, while elements on the walls will flow

by at the same rate for short and tall observers (i.e., the wall is the same distance

from short and tall observers while the floor is a different distance from short

and tall observers' eyes). Thus, rather than coding distance in terms of the angle

subtended by an extent in the environment, distances may be coded in terms of

the number of texture elements (i.e., the number of texture elements that flow by

for a given number steps for a treadmill virtual reality environment). In canonical flow fields, these two factors-angle subtended by an extent

on the ground and the magnitude of optic flow on the ground plane-co-vary as 
a function of egeheight. In order to disambiguate these two potential visual in

fluences. We manipulated both the effective eyeheight of a perceiver with no

change in (global) optic flow velocity in the display and the optic flow velocity

in the display to produce an equivalent magnitude of optic magnitude on the

ground plane for a fixed eyeheight. Method Participants walked on a treadmill while wearing a virtual reality head

mounted display (HMD), which depicted the observer traveling through a tun

nel. The task was to walk a target distance that would be indicated by a pair of

"starting position" cones and a pair of "ending position" cones (12 meters apart)

depicted in the graphical display, and then reproduce that distance (i.e., magni

tude production) on the next trial. Magnitude of optic flow on the ground plan for a given optic flow velocity

and eyeheight was determined according to the following equation (Warren,

1990): - V : I fJ = cos Q: 'sin f.J h.

where $v$ is speed, h is eyeheight, $a ; '$ specifies the meridian, and 33 specifies the

eccentricity of the line of sight (both $=90^{\circ}$ for looking straight down at the

ground), and 33 (dot) is the flow magnitude expressed as a rate of change of an

gular position. Magnitude of optic flow on the ground plane was manipulated by

pairing five levels of eyeheight $(1.20,1.10,1.0, .90$, and $.80 \times$ actual height de 
picted at $1.8 \mathrm{~m}$ ) with an optic flow velocity of $1.12 \mathrm{mls}$ (2.5 mph) yielding five

values of optic flow magnitude defined with respect to the ground and the ob

server's point of observation $(0.51,0.56 ; 0.61,0.68$, and 0.76 degls, respec

tively). Optic flow velocity was manipulated accordingly $(1.42,1.25,1.12,1.01$,

$0.93 \mathrm{mis}$, respectively) with no corresponding change in eyeheight to achieve

the same values of optic flow magnitude on the ground plane as in the eyeheight

manipulation conditions. A constant treadmill (Le., actual) walking speed of

$1.12 \mathrm{mls}$ (2.5 mph) was used in all conditions, yielding 9 experimental condi

tions. Repating clstar loe Figure 1. Method of presenting standard distance and reporting distance via magni

tude production.

Results and Discussion Distance reports were submitted to a 5. (Magnitude of optic flow) $\times 2$ (Mode

of manipulation) repeated-measures analysis of variance. There was no main

effect for magnitude of optic flow and no main effect of mode of manipulation,

Fs < 1 . There was, however a significant interaction between the two factors,

$F(4,44)=2.63, P<.05$. Post-hoc comparison showed that mode of manipulation

influenced perceived distance at the optic flow magnitude of 1.1 , units, $t(11)=$

$2.67, p<.05$, with the optic flow velocity manipulation showing a greater per 
ceived distance than the eyeheight manipulation .

.-. 1.:::::.g(1) 12 u c: 10 Co

88

"0 $6 \% \mathrm{~L}$

$105 \sim 1$ ( 1$)$

ด... ด tvu(j $j^{\sim}$ ur Mar Ii fJulc:lliu.1 - beget lei ${ }^{*}$ t Il - . optic Flow Velocity $1.03 \quad 1.131 .251 .-11.6$ Magnitude of optic Flow (rad/s) Figure 2. Distance perception as a function of magnitude of optic flow on the

ground plane with optic flow velocity and eyeheight as modes of manipulation. Neither manipulation of optic flow magnitude showed a systematic influ

ence on distance perception. However, a decrease in optic flow velocity did

show a significant increase in distance perception relative to the eyeheight ma

nipulation for one magnitude of optic flow, which is consistent with previous

studies showing that perceived distance increases with decreasing optic flow

velocity (e.g., Proffitt et a!., 2003; Mohler et a!., 2004; White, 2008). The lack of

a systematic influence of optic flow velocity may reflect the fact that the present

study used a narrower range of optic flow velocities that other studies showing

an influence of that variable. The present study shoued no evidence that eye

height influences egocentric distance reports in contrast to the results of $00 i$ and

Mark, L. S. (1987). Eyeheight-scaled information about affordances: A study of sitting and stair climbing. Journal of Experimental Psychology, 13, 367370. 
Mohler, B. J., \& Thompson, W. B. (2007). Calibration of locomotion resulting from visual motion in a treadmill-based virtual environment. ACM Transaction on Applied Perception, 4,1-15 ..

Doi, T. L. \& He, Z. 1. (2007) A distance judgment function based on space perception mechanisms revisiting Gilinsky's (1951) equation. Psychological Review, 114(2), 441-454.

Proffitt, D. R., Stefanucci, 1., Banton, T., \& Epstein, W. (2003). The role of effort in perceiving distance. Psychological Science, 2, 106-112.

Rieser, J. J., Pick, R. L., Ashmead, D. R., \& Garing A. E. (1995). Calibration of human locomotion and models of perceptual-motor organization. Journal of Experimental Psychology: Human Perception and Performance, 3, 480497.

Warren, W. R., Jr., \& Whang, S. (1987). Visual guidance of walking through apertures: Body-scaled information for affordances. Journal of Experimental Psychology: Human Perception and Performance, 13,371-383.

White, E. (2008,) The influence of multimodally specified effort on distance perception. Unpublished master's thesis, University of Cincinnati, Cincinnati, ohio, USA. Wraga, M., \& Proffitt, D. R. (2000). Mapping the zone of eye height utility for seated and standing observers. Perception, 29, 1361-1383. Acknowledgements. This research was supported by NSF grant \#0716319. 


\section{Chapter 4: Perception of Affordances}

Davis, T. 1., Ramenzoni, V. C., Shockley, K., \& Riley, M. A. (2008). Tuning in to another agent's action capabilities. 'Cogsci 2008: Proceedings of the 30 th Annual Meeting of the Cognitive Science Society.

Fajen, B. R., Riley, M. A., \& Turvey, M. T. (2009). Information, affordances,

and the control of action in sport. International Journal of Sport Psychology,

$40,79-107$.

Gibson, J. J. (1979). The ecological approach to visual perception. Boston,

Houghton Mifflin

Proffitt, D. R. (2006). Embodied perception and the economy of action. Perspec

tives on Psychological Science, 1, 110-122.

Ramenzoni, V. C., Riley, M. A., Shockley, K., \& Davis, T. (2008). Carrying the

height of the world on your ankles: Encumbered observers reduce estimates

of how high an actor can jump. Quarterly Journal of Experimental Psychol

ogy, $61,1487-1495$.

(9) 2009 Taylor \& Francis Group, LLC Understanding the Dynamics of Behavioral Transitions in Affordance Experiments Stacy Lopresti-Goodman\T.D. Frank M. J. Richardson2, \& M.T. Turveyl iCESP A, University of Connecticut, Storrs, CT lCoJby College, Wflterville, ME To perceive an opportunity for action presumes that one perceives the envi

ronment relative to one's dimensions and capabilities (Gibson, 1979/1986). For

example, an object that affords grasping with one hand by an adult, affords 
grasping with two hands by a small child. Despite

different action capabilities of

differently sized individuals, an invariant

object-size/hand-size ratio (a $1 t$

number) seems to specify the boundary between oneto

two-hand grasping. It is

the case, however, that the boundary differs experimentally

for systematically

increasing and systematically decreasing object lhand

ratios. There is hysteresis

(Lopresti-Goodman, Richardson, et aI., 2009). Here we derive parameter estima

tions for a dynamical model (Frank, Richardson et aI., in press) of this hysteresis

effect which is based on a model first introduced by Haken (1991). Let the order parameters ${ }^{*} I$ and ${ }_{2}$ represent the generalized amplitudes of

the oneand two-hand grasping modes, respectively. " I $>0$, $\sim_{2}=0$ defines the

one-hand mode, ${ }_{2}>\theta,{ }^{\infty} I=0$ dermes the two-hand mode. Then, the grasping

behavior is determined by the time evolution of ${ }^{\circ} \mathrm{I}$ and $\sim_{2}$ :

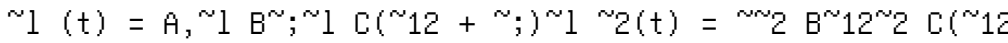
$\left.+{ }^{\infty} ;\right)^{\infty}$ (1) (2)

In (1) and (2), $A \mathrm{~L}$ and $\mathrm{Az}$ are the oneand two-hand (so-called attention) parame

ters, respectively, corresponding to ${ }^{\sim} \mathrm{I}$ and $\sim_{2}^{\prime} \mathrm{By} \mathrm{Eq} .12$ in Frank et $a I$., $\mathrm{Al}$ and $\mathrm{AZ}$

relate linearly to the object lhand ratio (a) which acts as the control parameter

(van der Kamp, Savelsbergh, \& Davis, 1998). Al = ]3(L I , 0 a) $A z=] 3(L 2,0+a)(3)$ (4)

where $f . J$ detennines the overall size of A.. From Eq. 9 in Frank et $a$ !. we see that $B=C(g-1)$ 
With some simple substitutions, Eqs. 1 and 2 become

$\sim j(t)=A,{ }^{\prime} ; j-\operatorname{Cg}^{\prime} ;{ }^{\prime \prime} ; I-C^{\prime} ; j 3 \sim_{2}(t)=.1 .2^{\prime} ; 2 \mathrm{Cg}{ }^{\prime} ; j 2^{\prime} ; 2$

C.; ; (5) (6) (7)

with $g$ representing the strength of the interaction between the two grasping

modes and the stability oftheir attractors. . Estimates of $L 1,0, L 2,0$, and $g$ can be derived from Lopresti-Goodman et a!.,

(2009). They investigated the task of grasping and moving light wooden planks

of different sizes using either one or tuo hands. In their Experiment 2, they

found that speed of object presentation (Slow: 10s/plank and Fast: $3 \mathrm{~s} /$ plank),

and cognitive load (Load: counting backwards aloud by 7 , or No Load: Not

counting) affected the degree of hysteresis. Given the physical constraint at $a=1$ in the ascending sequence, forcing a

switch from oneto two-hands, we will set $L 1,0=1$.

Parameter values for $L 2,0$ and

g can then be derived from Lopresti-Goodman et al. 's experimental observations

of the transition points for the ascending $(a, e, 2)$ and descending ( $a$ e , ]) sequences.

Eq. 13 of Frank et a!. yields (8)

Furthennore, a detailed analysis shows (see Eq. 10 below) that $g$ increases when

the difference between the a c values ( $L$ la e =a e , 2 a c , ) increases, provided that

$L 2$, 0 is constant. That is, $g$ is related to degree of hysteresis. A qualitative analysis of the Lopresti-<; ioodman et al. data revealed that hys

teresis is exaggerated with increased constraints on the 
system. Accordingly, we

expect $g$ to be influenced by the level of task-difficulty, and should be larger for

those conditions in which participants experienced a fast presentation speed or a

cognitive load. A 2 (Speed: slow or fast) $\times 2$ (Cognitive Load: no load or load)

Univariate ANOVA revealed a significant effect of speed, $F(1,44)=6.28, P<$

0.05 , indicating that $g$ for the fast-paced condition (M= $1.91 \pm 0.85$ ) was signif

icantly larger than $g$ for the slow-paced condition ( $M=$ $1.37 \pm 0.60)$. There was

no significant differences for the different Cognitive Load conditions (MLad =

$1.79 \pm 0.69$ and Mo Load $=1.49 \pm 0.84$ ), nor was there a significant speed $x$

Cognitive Load interaction, $F<1.0$. Since' $g$ detennines the strength of the inte

raction between the two grasping modes, as well as the strength of the two mod

es' attractors, we may infer that increasing the speed of object presentation in

creases the interaction between the two grasping modes. With regard to the $L 2$, o parameter, an analysis of $E$. 13 of the Frank et al.

paper yields $105 \mathrm{~L} 2,0=1$ ac, 1 a $\mathrm{c}, 2$ (9)

Since $L 2$, 0 also depends upon the size of Liac. we expect task-difficulty to influ

ence its value. Specifically, when task-difficulty

increases, $L 2$, 0 may also in

crease indicating that the two-hand mode represents a more stable form ofbeha 
vior for more difficult tasks. Therefore, increased values of $L 2$ a should result in

participants using the two-hand mode more often, $A$ qualitati $i e$ analysis of the

Lopresti-Goodman et al. data revealed, however, that this is not the case. In

stead, the significant Sequence $\times$ Speed and Sequence $\times$ Load interactions ind $i$

cate that participants only used the two-hand mode more often in the descending

sequences. Specifically, ac, l decreased with increases in task-difficulty, whereas

a $c, 2$ was invariant regardless of the condition;

therefore we may not expect sig

nificant differences on $L 2,0$ for the different levels of task-difficulty. A Univariate ANOVA revealed that there was no effect of Speed, $F(I, 44)=$

2.79, $P>0.05$, Cognitive Load, $F<1.00$, nor an interaction between the two, $F$

$<1.00$. Despite the lack of significant differences, qualitatively $L 2$, 0 was larger in

the fast-paced $(M=-0.29 \pm 0.18$ ) and cognitive load $(M=$ $-0.27 \pm 0.17)$ condi

tions than in the slow-paced $(M=-0.34 \pm 0.20)$ or no load $(M=-0.36 \pm 0.21)$

conditions. These results suggest that $g$ and $L 2,0$ interact and simultaneously

change with increases in task difficulty. An analysis in the $g \times L 2,0$ two

dimensional space may reveal differences in where these parameter values clus

ter. For example, low levels of task difficulty may result in $g$ and $L 2,0$ values

clustering near the origin, while increased levels of task 
difficulty may result in

clusters farther from this point. Since we know that hysteresis size (Lia c) is in

fluenced by task difficulty, we will plot $g$ and $L 2$, 0 on contour lines for which

Lia $c$ is constant (Figure 1). Lia $c=(g 1)(1+L 2$ , o) $/(g+1)$ (10) For each data point, we have calculated the signed minimal distance from

the contour line representing Lia c > 0. A Univariate ANoV A revealed a signifi

cant effect of speed, $F(1,44)=6.87, P<0.05$, with the parameter values for the

slow-paced condition clustering below this line $(M=-0.09$ \pm 0.35 ) and the fast

paced condition clustering above it $(M=0.19 \pm 0.42)$. There was also a signifi

cant effect of Cognitive Load, $F(J, 44)=6.43, P<0.05$, with parameter values

for the no-load condition clustering below the contour line $(M=-0.89 \pm 0.43)$

and for the load condition clustering above it $(M=0.19 \pm$ 0.37). There was no

significant speed $x$ cognitive Load interaction, $F<1.00$. In conclusion, we have demonstrated with the parameter derivations for the

Frank et al. model that affordances fit within a dynamical systems frameuork.

Additionally, we have shoun that differing levels of task difficulty influence the

interaction between the two possible grasping modes and the strengths of their

corresponding attractors. Future empirical work will focus on testing predictions

the model makes about the influence of other levels of 
task difficulty. $430 \_0.8-0.6-0.4-0.200 .2 \quad 0 \quad 2$

, Q Figure 1. Solid black circles represent participants in the slow-speed no-load condi

tion, solid white the slow-speed cognitive condition, black diamonds the fast-speed no

load condition and $x s$ represent the fast-speed cognitive condition. The first contour line,

Frank, T. D., Richardson, M. 1., Lopresti-Goodman, S. M., 8 Turvey, M. T. (in press). Order parameter dynamics of body-scaled hysteresis and mode transitions in grasping behavior. Journal of Biological Physics.

Gibson, I. I. (1979). The ecological approach to visual perception. Boston: Houghton Mifflin.

Haken, H. (1991) Synergetic computers and cognition, Springer, Berlin

Lopresti-Goodman, S. M., Richardson, M. I., Baron, R. M. Carello, c., \& Marsh, K. L. (2009). Task constraints and affordance boundaries. Motor Control, 13,69-83.

van der Kamp, I., Savelsbergh, G. I. P., \& Davis, .. E. (1998). Body-scaled ratio as a control parameter for prehension in 5to 9-year-01d children. Developmental Psychobiology, 33(4), 351-361.

(9) 2009 Taylor \& Francis Group, LLC Differences between American and

Chinese Students in Perceiving Multiple Affordances for Common objects Lin Ye, Wilson Carduell, Stephanie Karges, Milena Petrovic, \& Leonard S. Mark Department of Psychology, Miami University, oxford, ohio Previous work has shown that perceiving one of an object's nondesigned

(i.e., not the primary use for which an object was designed) uses reduces the

like lihood of detecting another non designed use for that object (Ye, et al., 2006).

Participants were shown a collection of nine objects. Some objects had only

affordance I (OAFF 1) (e.g., pound-able-with); other objects had only affordance 
2 (e.g., scoop-able-with) (DAFF 2); the remaining objects had both affordances I

and 2 (OAFF 182). For Task I, participants identified all of the objects with affor

dance 1. Immediately following the completion of the first task, participants

performed Task 2, in which they identified objects with affordance 2, which

included objects with both affordances as well as objects with only affordance 2 .

Ye et al. (2006) found that on Task 2, participants were more likely to identify

objects with only affordance 2 than objects with both affordances that had pre

viously been identified as having affordance 1 on the first task. In a follow up

study with students from china who were studying in the United States, the dif

ference between DAFF2 and DAFFI\&2 was significantly smaller on Task 2 than for

American college students. Figure 1 summarizes these initial findings. This dif

ference was not unexpected because in the' Chinese culture people typically find

multiple uses for a single object, unlike in the United States, where people tend

to favor special purpose tools that are optimal for performing a particular func

tion. For example, whereas Americans have special

implements for holding

com, the chinese use their chop sticks for a variety of functions, including serv

ing as "com holders." A recent Asian visitor to our lab 
commented that, "The

Chinese people use chop sticks for just about everything." The current study examines the following problem: Inder what conditions

people are most likely to notice a second 'affordance for an object? A complex

act, such as scooping up rice with a cup, entails grasping the cup to be used on a

particular part of the cup, using a particular grip such that the cup can be

wielded in such a manner as to allow rice to enter and be maintained in the cup.

In effect, there is a nesting of affordances entailed in performance of a complex

goal-directed action. We suggest that when the object is grasped using the same

grip in order to perform both goal-directed actions, people will be more likely to

recognize the second use for the object than when different grips is required for

both actions. $1 \mathrm{~m} 80.00 \sim \sim$ (.J $\sim$ p., 40 D0;000

0.00 Chinese Anl eri ca 11 o oafiz . odl2 Figure 1.

The percentage of DAFF2 and DAFF 182 identified on Task 2 by stu

dents from china and the United States. Method Twenty-eight Miami University students participated in this study. Half of

the students were from china and the remainder were from the United States.

There were two collections of roughly 15 objects each. The first set of objects

contained objects consisting of two affordances: Some of the objects could only

be used to dig a small hole to bury a baseball in sand with; other objects could 
only be used to cut Play Doh into strips; still other objects could be used to per

form both actions (0 AFF182). The second set of actions was scooping rice from a

container and crushing (pounding) a cracker into crumbs. Again, objects either

afforded one or both of those actions. Before being instructed about the nature of the first action, participants were

given the opportunity to pick up the various objects in front of them. Once the

instructions for the first action were given, participants were then allowed to

pick up each of the objects that could be used to perform that action and demon

strate how they would use it by actually performing the action (Task 1). This

procedure was repeated for the second action (Task 2). After completing the 109

second action, the experimenter asked the participant to demonstrate how

a AFF 182 that had not been picked up on task 2 , but had been picked up on Task 1 ,

might be used to perform the second task. These objects were treated as

"missed" objects. The order in which participants performed the two actions was

counterbalanced. The actions were videotaped and digitized. Two experimenters coded the

grip used for each action with the DAFFl82 objects for which the first action had

been identifled. To code the grip. the coders used Newell et al.'s (1989) prehen 
sion taxonomy and noted the location on the object where the object was

grasped when performing the required action. For the pair of actions used for a

given object to be counted as "same grip," both the number of fingers and their

placement on the object had to be identical. The number of same grips for ob

jects that were spontaneously used to perform both actions was recorded as well

as the number of different grips used when an object was not used spontane

ously for the second action. This allowed us to determine the number of correct

predictions (same grip when both actions were performed spontaneously and

different grip when only the first action was performed spontaneously.) Results and Discussion

Chinese students objects (AFFl\&2) Missed objects

American students Used for Both Actions (AFFl82) for the Second Action 6116 Same Grip 7362648 Different Grip 2947 Table 1. For the Chinese (bold) and American (italics) students the fre

quency counts for spontaneously using OAFFle2 for both actions and missing the

DAFFl 82 for the second action. Each of these frequency counts is broken up into

those in which the same grip was used in both actions and those in which differ

ent grips were used. The data are summed over the two pairs of actions. Table 1 shous a summary of the frequency data for this experiment. Dur

hypothesis was that people would detect both affordances in objects that would 
be gripped the same for both actions and would fail to

detect the second affor

dance when different grips were used. Thus, correct

predictions appear in the

upper left and lower right cells respectively. 0verall, we correctly predicted

whether an 9AFFl\&2 would be used for both actions or only for the first action for

72.28 and $77.4 \%$ of the objects for the chinese and American students respec

tively. The percentages for the chinese and American students for the dig-cut

actions were 75.08 and 80.78 and for the pound-scoop actions, $67.8 \%$ and

73.6\%. We detennined for each participant the proportion of correct predictions and

constructed a t-test to ascertain whether the percentages were significantly dif

ferent from chance (50\%): For the chinese students, t (26 [due to camera mal

function]) $=5.95, P<.001$; for the American students, $t$ $(27)=3.43, P<.002$. There are several findings of interest. First, our pilot study comparing the

perfonnance of Chinese and American students obtained a significant difference

in the percentage of 0 AFF 182 identified on Task 2 , compared to the percentage of

OAFF2. That study involved only judgments of which objects supported the ac

tions in question. The current study did not obtain a difference between the two

groups of students. This may be the result of having participants actually per

form the action. The task of perfonning the actions may 
pants in picking up infonnation relevant to the action being studied. In the earli

er study of perceptual judgments, the chance to explore these object properties

was minimal. Second, these results provide some support for the idea that a complex af

fordance, such as using an object as a tool to perfonn an action like scooping

rice from a container and placing it into <; bowl, entails detecting a nesting of

affordances, each of which must be perceived and utilized in order to complete

the goal-directed action. Each of the objects has to be grasped by a particular

part using a particular grip so that it can be wielded appropriately to perform the

intended action. Dur results show that when the actor uses the same grip to per

fonn both actions, the actor is more likely to perceive and act upon both affor

dances than when a different grip had to be used. But, actors did use a different

grip on 298 of the cases where the same object was used to perfonn both tasks.

This suggests that another factor is involved in detecting the second affordance

of an object. In this regard we have to consider the possibility that how the ob

ject is wielded when perfonning the two actions may also contribute to the de

Newell, K. M., Scully, D. M., Tennebaum, F., Hardiman, S. (1989). Body scale and the development of prehension. Developmental Psychobiology, 22, 114. 
Ye, L., Mark, L. S. \& Taliaferro, M. (June 2006). The perception of multiple affordances. Paper presented at the spring Meeting of the International society for Perception and Action. Cincinnati, ohio. Acknowledgements. This research was supported by the Committee for $\mathrm{Fa}$

culty Research at Miami University.

(9) 2009 Taylor \& Francis Group, LLC Changes in Anterior Deltoid Muscle Activity at the Preferred Critical Boundary for Reaching Milena Petrovic, Adam Strang, \& Leonard S. Mark Department ofPsycho!ogy, Miami University, oxford, $\mathrm{OH}$ To support a particular action mode' (pattern of coordination among the

limbs and torso), relevant environmental properties must be within a range de

termined by the action being performed and the prospective actor's body scale

and biodynamic capabilities. Beyond that range the environment no longer af

fords the action mode and the prospective actor must engage another mode to

achieve the intended goal. Research on reaching (Choi \& Mark, 2004; Gardner

et aI., 2001; Mark et aI., 1997) has shown that when not required to use a par

ticular reach mode, the transition from one reach mode to another (e.g., arm

only reach to an arm-and-torso [lean] reach) occurs not at the absolute maximum

distance at which an arm-only reach mode could be used, but at slightly closer

distances, what Mark et aI. (1997) referred to as the preferred critical boundary.

What is responsible for the location of the preferred critical boundary? Several studies have found that a change in the relative "comfort" of the 
competing action modes coincides with the location of the preferred critical

boundary (Mark et aI., 1997; Stasik \& Mark, 2005). This raises the possibility

that actors attempt to avoid aukward postures and extreme levels of muscle ac

tivity. The objective of the current study is to examine levels of muscle activity

in the anterior deltoid (a superficial shoulder muscle involved in arm extension)

while reaching at distances near the preferred critical boundary. We expected

that muscle activity would increase as a function of distance up to the preferred

critical boundary. However, when participants were not restricted on how to

reach, the rate of increase was expected to change (decrease) at or near this

boundary. In contrast, when they were required to use an arm-only reach, we

expected the rate of increase of anterior deltoid activity to either increase or re

main the same at the preferred critical boundary. Method Six men and 9 women students (age 18-34 years) participated in this study

in partial fulfilment of a course requirement.

Participants were seated on a

height-adjustable ergonomic chair placed in front of a height-adjustable table.

Table and chair height and table-to-chair distance were set relative to the body

scale of each participant using the procedures followed in earlier work (e.g.,

Mark et aI., 1997). Activity of the anterior deltoid 
muscle of the shoulder was

measured using surface electromyography (EMG) recorded with 12-bit resolu

tion at a bandwidth of $10-500 \mathrm{~Hz}$ and amplified 1000 times. Each participant's absolute critical bolundary for an arm-only reach was de

termined by procedures followed in earlier reach studies (Stasik \& Mark, 2005),

where the target object was placed at increasing distances until the participant

could no longer pick up the object using an arm-only reach. The reach distances

were determined from 0.651 .20 of the distance of the maximum arm-only

reach in increments of 0.05. In condition 1, participants were instructed to reach in a manner that they

found most comfortable. That is, there were no restrictions imposed on partici

pants as to how to reach. For condition $2 \cdot$ the same participants were instructed

to use only an arm-only reach; this meant that reaches beyond 1.0 could not be

completed. In each condition, participants reached three times at each distance

in random order. The order of conditions was not counterbalanced because of

our concern that performing the arm-only reaches in condition 2 might influence

the measure of the preferred critical boundary. Results and Discussion for condition 1, the location of the preferred critical boundary for each par

ticipant was determined graphically by plotting the percentage of arm-only

reaches as a function of distance. The preferred critical 
boundary was identified

as the distance at which the function passed through the $50 \%$ level. The average

distance of the preferred critical boundary was roughly 0.95 of the maximum

arm-only reach, a finding comparable to those in previous studies. Figure 1

shous the average maximum amplitude of anterior deltoid activation as a func

tion of reach distance. We determined the slopes of the function from $0.65-0.95$

(slope $=10.6, \mathrm{SE}=0.96)$ and from 0.951 .20 (slope $=2.59$, SE 1.48). An

ANCova showed that these slopes were significantly different, $F=18.2,(1$,

147), $P<.001$, indicating that the change-in reach mode modulated the rate of

increase in anterior deltoid activation. 1138.07 .0

slope $=2.596 .0 \ldots$

15.0 - EMG (Condition 1 before PCE)

(1) 4.0

$::: ! \mathrm{E}$

W 3.0 i EMG (Condition 1 after PCB) 2.0 --Linear (EMG (Condition 1 before PCEM 1.0 Linear (EMG (Condition 1 after PCB 0.0

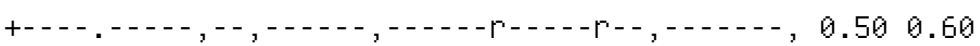

0.700 .800 .901 .001 .101 .201 .30 Distance (absolute critical boundary) Figure 1 . The average maximum amplitude of anterior deltoid activation as a func

tion of reach distance is depicted by the location of the markers. The diamond and trian

gle markers denote reach distances closer and farther respectively than the location of the

preferred critical boundary. The solid line depicts a 
closer than the preferred critical boundary. The broken line shous the linear regression

for the distances beyond the preferred critical boundary.

9.00

8.00 - EMG(Condition 1)

7.00 - EMG(Condition 2)

6.00

$\sim 5.00$

(1) $4.00: \mathrm{iE}$

UI

3.00

2.00

1.00

$\begin{array}{llllllllll}0.00 & 0.50 & 0.60 & 0.70 & 0.80 & 0.90 & 1.00 & 1.10 & 0 \text { Distance }\end{array}$ (absolute critical boundary) Figure 2 . The average maximum amplitude of anterior deltoid activation as a func

tion of reach distance is shoun for conditions 1 and 2 . Muscle activation was not signifi

cantly greater for condition 2 in which participants had to use an arm only reach. There

was no marked change in condition 2 at the location of the preferred critical boundary. For condition 2 in which participants used an ann only reach, the pattern of

EMG activity was similar to that obtained in condition 1. Figure 2 shous that

while the level of anterior deltoid activity increased up to the preferred critical

boundary, the overall level of activity in condition 2 was not significantly high 
er than in condition I (free reach), $F=0.36,(7,210), F=$ 0.93 . It is possible that

the restriction to reach using only an ann-only reach may have caused some

additional muscle activation (tension) in condition 2 . The outcome of condition 1 suggests that actors attempted to maintain the

level of anterior deltoid activation below a certain level by suitching from an

ann-only reach to an ann-and-torso reach (at the preferred critical boundary).

However, the pattern of activation obtained in condition 2 suggests that anterior

deltoid activity does not increase much beyond the level obtained at the pre

ferred critical boundary. It may be the case that the additional reach distance

(from $0.95-1.00$ absolute critical boundary) does not provide sufficient opportu

nity for much increase in anterior deltoid activity. More likely, other muscles

may have played a significant role in extending the reach capabilities. This

points to the need for a future study to obtain satisfactory signals from other

muscles that may be involved in reaching, such as upper trapezius, erector spi

nae and superspinatus. The results of this study do raise the possibility that

actors attempt to maintain the level of anterior deltoid activation at a submaxim

al level when given the opportunity to do so. Future work must devise a more

subtle method for probing these differences in muscle 
activation around the pre

Choi, H. J., \& Mark, L . S. (2004). Scaling affordances for human reach actions. Human Movement science, 23, 785-806.

Gardner, D. L., Mark, L. S., Ward, 1. A., \& Edkins, H. (2001). How do task characteristics affect the transitions between seated and standing reaches? Ecological

Psychology, 13,245-274.

Mark, L.S., Nemeth, K., Gardner, D.; Dainoff, M. J., Paasche, J., Duffy, M.; Grandt, K. (1997). Postural Dynamics and Preferred Critical Boundary for Visual Guided Reaching. Journal of Experimental Psychology, 23(5), $1365-1379$.

Stasik, S., \& Mark, L. S. (2005). Comfort as a detenninant of the location of critical boundaries in the act of reaching. In $\mathrm{H}$. Heft \& K. Marsh (Eds.) Studies in Perception and Action Vll. Mahwah, New Jersey: Erlbaum Associates. [pp 23-27].

1. B. Wagman \& C. C. Pagano (Eds.)

(6) 2009 Taylor \& Francis Group, LLC

Development, Balance and Flexibility as Constraints on Perceiving Affordances Peter B. Pufall \& Kathryn Hobbs Smith college In previous research (Pufall \& Dunbar, 1992) we extended Warren's (1984)

similarity hypothesis explaining individual differences among adult humans

perception of affordances to development.. That is, we hypothesized that child

ren's perception of $f$ it between the limits of actions of stepping onto and over

obstacles scattered over their surface of action would be invariant over devel

opment because the grouth rates in the upper and lower legs were constant thus

fixing a skeletal invariant in the biomechanical suing of the legs for each step

ping action. The optical flow of light reflectively 
world would be structured with respect to self by the invariant suinging struc

ture of our gate and stepping actions. The extended similarity hypothesis was confirmed. Six through I0-year-old

children accurately perceived the critical limits of both stepping actions. The

purpose of the present research is tuofold. One, we are testing whether or not

the developmental-similarity hypothesis can be extended developmentally to 4

year-old children. These children have the same skeletal leg proportions as old

er children and adults, hence there is reason to expect that they would they

would accurately perceive these affordances. However, they differ dynamically

from older children, and in particular in terms of static balance. Two, we are

exploring whether biomechanical features affect perception. Four-year-old

children cannot maintain their static balance as effectively, that is, as long, as

older children. If the optic flow of information is affected by the self regulated

balance of the body, then these children may not perceive a tight fit between

their actions and the heights of objects within their world. In particular it may

be difficult for them to perceive the critical limits of affordances. In addition,

we are exploring whether or not the flexibility of hip and knee joints, joints cen 
tral to stepping, are related to perception of stepping affordances. Method Half of the 4-, 6-, 8and 10-year-old children were tested in the stepping

Onto and half in the stepping over condition. In both conditions children iden

tified the maximum height $(M H)$ of a bar over or onto which they could step.

The child stood 10 feet from the testing apparatus, which consisted of a uooden

bar that was moved up and doun a vertical pole to which it was attached. On

half of the Stepping trials the experimenter, behind an opaque screen that also

hid the vertical bar, moved the bar up (Ascending trials) from well below the

child's MH and on the other half she moved the bar down (Descending trials)

from well above the child's MH. Each child completed 3 ascending and 3 des

cending trials in a randomized order. The child said "stop" at the PlH onto or

over which she could step. After the Perceived Stepping trials the children Actually stepped onto or

over an increasing number of stacked boards. The Actual Maximum Height

(ANH) was the height of that stack before the child failed to successfully per

form the action. Perceived Critical Limits ( $\mathrm{PCL}$ ), a measure of whether children

accurately perceive the limits of their stepping functions in terms of heights of

obstacles, is the average ratio of the $\mathrm{PMH}$ to the AMH over all six trials. A ratio 
of 1.00 indicates that $\mathrm{PMH}$ is equal to $\mathrm{AMH}$, and

theoretically, that children

perceive precisely what the affordances of these stepping actions are. Static balance was the number of seconds a child could stand on one leg.

Flexibility of the hip joint was the angle formed by the upper leg and the vertical

line of the body when the child folded one leg up into the upper body; flexibility

of the knee was the angle between the upper and lower leg when the latter was

bent as far as possible toward the upper leg. Greater flexibility is directly re

lated to the degree of each bend. Results and Discussion As expected, children's PMH varies directly with age $\mathrm{F}(3,47)=44.22, \mathrm{p}<$

.000), that is, with increasing age children's PMH is significantly higher than it

was the year before $(p<005)$. In contrast to the prediction from our similarity

model PCL varied with age $(F(3,47)=6.95, P<.001)$; post hoc analyses indi

cate the age effect is reflected only in the differences between the youngest

children's estimates and those of the three age older groups $(\rho<.007)$. The lat

ter finding replicates our previous work, while the former indicates that the

model cannot be generalized to children younger than age 6 years. More pre

cisely, because the proportional relation of leg to louer leg is an invariant across

the full age range tested it appears that the geometric constraint set by children's 
skeletal structures is not sufficient for them to perceive accurately the critical

limits of stepping affordances. Children at all ages overestimate the height of

obstacles they can step onto more than they overestimate the height they can

step over $(F(3,47)=6.95, P<.001)$. In the absence of a significant interaction

between age and stepping actions, it appears that the extent of this difference

persists over development. 117

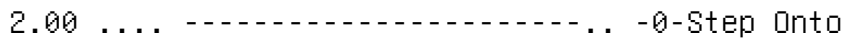

$1.50 \ldots \ldots+-., \ldots,-\cdots-1$

ด. "ด $1-\ldots \ldots$

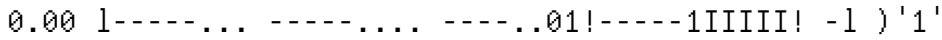

$01<16^{\sim 1} 1$ ' old 8 yr old $\left.1 \%\right) 1$ ' on Chronological Age:

Figure 1: Mean Perceived Critical Limits expressed as the ratio of children's PMH to the AMH of obstacles onto or over which they can step. Children at all ages perceived a higher MH on trials when the bar was des

cending than when it was ascending $(\mathrm{F}(1,48)=78.44, \mathrm{p}<$ . 000). When this dif

ference is represented by DPCL and APCL, it is clear that children's overesti

mations of the CLs is due primarily to their judgments on the descending trials

(Figure 1). The fact that children perform so well on the ascending trials yet

overestimate on the descending trials over development and across stepping

functions raises the possibility that descending trials generate error linked to our

method. To be sure, asking children to perceive a critical limit with respect to a 
moving an object challenges the ecological validity of the psychophysical me

thod we used, however, the fact that they accurately perceive their limits on one

kind of trial and not another suggests that our method should be examined not

because of movement but the direction of movement. On ascending trials the

bar is first situated with respect to the surface of locomotion and affords both

stepping actions. By contrast on the descending trials children may not initially

perceive it with respect to the surface locomotion and it is beyond the limits of

the actions by which we are asking children to scale their physical world. That

is, the descending trial is less ecological than the ascending trial. Even if this aspect of our method is a contaminant, it does not account for

the fact that the four-year-old children do not perceive what their world affords

as precisely as older children do even on the ascending trials. We hypothesized

that children may vary in the accuracy with which they perceive stepping affor

dances because of constraints on their skeletal structure. Children did not vary

over development with respect to hip or joint flexibility, but they differ signifi

cantly with respect to static balance $(F(3,52)=8.72, p<$ .000). The youngest

children's static balance time was significantly less than the static balance of the 
other three age groups $(p<.005)$. That is, the abrupt development between 4

and 6 years of age in children's perception of CLS is mirrored in their develop

ment of static balance. Moreover, correlations between age and balance $(r=$

$.52, p<.000)$ are significantly correlated. The negative correlation indicates

that the longer children maintained their static balance the more accurately they

perceived the fit between stepping actions and whether or not the height of ob

jects are within the limits of affording those actions. In sum, these findings suggest that the similarity model needs developmen

tal fine-tuning. Although young children share the same skeletal proportions of

leg length to upper and lower leg detecting this invariant relation the geometry

of the leg and actions within the world is built from moving through the world

and detecting the flow of visual information specific to forms of action. The

fidelity of this information appears to depend on static balance, at least, and per

haps dynamic balance. Static balance may have both a developmental role as

well as account for individual differences in older children's perception of af

Pufall, P. B., \& Dunbar, C. (1992). Perceiving whether or not the world affords stepping onto and over: $A$

developmental study. Ecological Psychology, 4(1), 17-38.

Warren, H. H. (1984). Perceiving affordances: Visual

guidance of stair climbing. Journal of Experimental

Psychology: Human Perception and Performance, 10,683-703. 
(9) 2009 Taylor \& Francis Group, LLC Ecometrics of Step-over Height Masaki Veno, Shogo Hirata, Hideyuki okuzumi, \& Mitsuru Kokubun Tokyo Gakugei University Ecometrics is one of the central themes of ecological psychology (Warren,

1984). In this study, we investigate the choice of actions when walking through

an obstacle, that is, when adults choose stepping-over or passing-under a rope,

focusing on the relationship between choice and the subject's height. In addi

tion, we also measure school-age children to investigate the developmental

trends of ecometrics. Method The subjects were 41

adults (23 males and 18 females) and 14 children aged

10 years (7 males, 7 females). Adults ranged from 19 to 30 years of age $(22.29 \pm$

2.18 years); the height of the males range ${ }^{\sim}$ from 160 to 183 cm $(170.13 \pm 5.22$

$\mathrm{cm})$, while the females ranged from 148 to $165 \mathrm{~cm}$ (157.50 \pm $5.12 \mathrm{~cm})$. The

height of the male children ranged from 128 to $147 \mathrm{~cm}$ $(135.29 \pm 6.97 \mathrm{~cm})$; the

female children ranged from 130 to $146 \mathrm{~cm}$ (137.57 \pm 6.48 cm). We devised an apparatus that could draw a rope tight, and allowed the rope

height to be adjusted between $20 \mathrm{~cm}$ to $130 \mathrm{~cm}$ from the floor. Subjects stood 2

$m$ in front of the rope, and were asked to walk beyond the rope by stepping-over

or passing under it. The rope height was changed up and doun in $5 \mathrm{~cm}$ incre

ments from the subject's waist, and presented at random. At each rope height, 
we recorded whether subjects stepped over or passed under, and whether they

hit the rope while doing so. We defme the height of the highest stepping-over rope as the "transition

point." That is to say, subjects pass under the rope that is higher than the transi

tion point. In this study, to analyze the relationship between the transition point

and the subject's height, we calculate a 'transition ratio" by dividing the transi

tion point by the subject's height. We use the transition point and transition

ratio for the analysis. Results and Discussion Table 1 shous the means and standard deviations of the transition point and

transition ratio in both age groups for both sexes. The transition point for adults

was obviously higher than that for 10-year-olds. In addition, while the transition

point in adult males is higher than that of females, the difference by sex was not

obvious in children. Transition point was analyzed by a two-way (age $x$ sex)

ANOV $A$, which demonstrated a significant effect of age (F1, $51=73.64, \mathrm{P}(.05)$,

no significant effect of $\operatorname{sex}(F 1,51=2.63, P>.05)$, but a significant interaction

$(F 1,51=7.09, P<.05)$. A simple main effect test demonstrated a significant ef

fect at the 58 level for a sex difference only in adults. These results show that

the development of task performance in this study is a process in which the tran

sition point rises with age, and the difference by sex 
emerges as males and fe

males begin to differ in height. Interest ${ }^{\circ} g l y$, the transition ratio in each age

group was almost the same regardless of sex. That is, all values are $0.4 x$. The

transition ratio was analyzed by a two-way (age $x$ sex) ANOVA, which shoued

no significant effects of age $(F 1,51=2.89, P>.05)$, $\operatorname{sex}(F 1,51=0.06, P>.05)$,

nor their interaction ( $F 1,51=0.64, P>.05)$. Therefore, the transition ratio is al

most the same in each group.

Table 1. Mean and standard deviation of transition point and transition ratio transition point transition ratio $M$ SD M SD Adults males 75.224 .730 .440 .03 females 68.17 $5.67 \quad 0.43 \quad 0.03$

curred at different points in adults and 10 -year-olds, the transition standard is the

same. If we describe the relationship between the transition point and subject's

height in the adults, the defining expression is as

follous: $T P / H=0.44(S D=.03)$ [TP: transition point, $H$ : subject's height].

That is, adults transition at a rope height that is 0.44 times their oun height. The

transition ratio value for 10-year-olds was the same as for adults. These findings

show a robust law of ecometrics in performing the task regardless of age. Mi

shima (1994) reported an expression of the transition of stepping -over and pass

ing-under based on leg length that indicated the same tendency as ours. Whereas

his study used a cognitive task to evaluate stepping-over 
and passing-under, our

study used a motor task. Note that since our study used a motor task, a few sub

jects touched the rope accidentally in each age group. subjects who touched the

rope accidentally, in effect, failed to accomplish the task safely. It remains to be

seen whether analysis of the relationship between the transition and its safety

can be refined. In addition, evaluating a wider age range of children would allow

Mishima, H. (1994). Perceiving affordances for suitching two actions, 'stepping-over' and 'passing-under'. Japanese Journal of Psycho logy. 64(6). 469-475. (In Japanese with English abstract.)

Warren, W. H. (1984). Perceiving affordances: Visual guidance of stair climbing. Journal of Experimental Psychology: Human Perception and Performance.1 o. 683-703. Acknowledgements. The authors would like to thank the participants who

made this work possible.

(6) 2009 Taylor \& Francis Group, LLC Investigating the Information Used To Detect An Affordance For Maximum Distance Throus ain zhu $1 \&$ Geoffrey Bingham 2 loivision of Kinesiology and Health, University of Wyoming, Laramie, USA

2Department ofPsychologicai and Brain Sciences, Indiana University, Bloomington, USA Previous studies (Bingham et aI., 1989; Zhu \& Bingham, 2008) have shown

that people with sufficient throuing experience were able to heft and select the

optimal weight for different sized graspable objects to be throun to a maximum

distance using their skilled throuing arm. The selected objects were indeed

throun to the farthest distances, indicating that throwing 
affordances of the ob

jects were well perceived. However, it remains unclear what information is

made available by hefting and used to detect throwing affordance. According to the ecological approach to perception and action (Gibson,

1986), information is required to both detect the useful structure of the environ

ment and assemble actions used to manipulate the environment. This assertion

leads to the smart perceptual mechanism hypothesis

(Runeson, 1977), in light of

which, hefting acts as a smart perceptual device to detect information about op

timal objects for throwing (Bingham et aI., 1989). Given hefting and throwing

are both actions performed with the throun objects, the objects must affect the

dynamics of both actions. Bingham et al. hypothesized that object size and

weight both affected the dynamics of hefting (size was found to affect the stiff

ness at the wrist, and optimal weight was assumed to control resulting frequency

of movement), and were likewise presumed to affect the dynamics of long dis

tance throwing. However, recent studies shoued that object size and weight did

not equally affect the dynamics of throwing (2hu $\&$ Bingham, 2008). object

weight was the only variable to affect throwing by constraining the release ve

locity), while object size only played role in projectile motion (after the object 
has left the hand) to affect the throwing distance. This finding invited further

investigation on the information underlying the perception of throuing affor

dance. 123 Experiment 1 we began our investigation by asking whether the information is intrinsic or

acquired. Considering that maximum distance throwing is a skill that has to be

learned, the perception of throwing affordances would presumably also be

learned, but if so, how? Perceivers could learn a single valued function (throw

ing distance) of two variables (object size and weight) through function learning

(Busemeyer et aI., 1993), or they could learn to direct their attention to the rele

vant information that reflects the inherent relationship between the environment

(object size and weight) and the result of action used to manipulate that envi

ronment (throwing distance), as predicted by the smart perceptual mechanism.

These two approaches can be distinguished using their respective requirements

for adequate sampling of the space. The former requires sampling of the entire

space, but the latter does not. Method Forty-eight spherical objects were made with eight weights in each of $\operatorname{six}$

sizes. The matrix of object size and weight was constructed so that three subsets

of objects could be created: a set of six objects of a constant weight, a set of six 
objects of a constant density, and a set of six objects of a constant size. One ob

ject was shared by all three subsets. Twenty-four unskilled adult throwers were

initially asked to heft all 48 objects and judge the optimal weight for throwing in

each size. Next, they actually threw every object in an outdoor field without

seeing the resulting distances of throus. Then, they started a month of throuing

practice, during which they were divided into 4 groups so that 3 groups prac

ticed throwing with vision using one of thtee sets of objects (constant size, con

stant weight or constant density). The fourth group used objects of constant den

sity without vision. After the month of practice, hefting judgments, throuing

(with vision) and then hefting judgments again were tested with the full set of

objects. Results and Discussion As shown in Figure 1, the preferred objects corresponded to those being

thrown to the farthest after practice, and this can only be seen in vision groups

independently of the practice set $(\mathrm{F} 1,15=41.98, \mathrm{P}<$ 0.001), indicating that per

ceptual information was discovered during learning to throw, and it was

achieved through a smart perceptual mechanism. The facts that the no-vision

group did not acquire the affordance perception and that all judgments became

better after seeing throus suggested that visual feedback is required for acquir 
ing the information about the affordance for throuing.

20.00

18.00

16.00

14.00

12.00

$-10.00$

.. ConDenw Vision Group Conwt Consz ConDen w/o Vision

$\sim 8.091$ $--^{\prime} \mathrm{L}-$ $\ldots . . \mathrm{J} \mathrm{L}$ $--\mathrm{J}$

GI

$\approx 20.00$

$t ; 18.00$

016.00

i 14.00

012.00

$.5:: .10 .00$

1

e $8.00, \ldots,, \approx ., 11$, c 'c

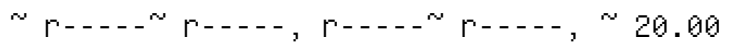

18.00

$16.00 \quad 14.00 \quad 12.00$

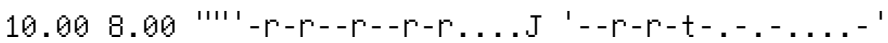

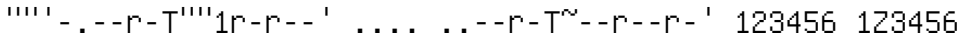
123456123456 Ball Diameter (Inch) > ; : : II . . . " "t. $\ldots, \operatorname{sol} n \ldots\left(;^{\prime} \ldots{ }^{\infty} \ldots \ldots\right.$, PREFEREI CE Preferred ..... Not Preferred. Pref rred a Not 
Preferred Figure 1 Mean throwing distances achieved by unskilled throwers after practice (separated for each group) as a function of size and preference across the hefting judgment phases. Filled circles connected with a solid line represent the preferred objects. Unfilled circles connected with a dashed line represent the un-preferred objects. Experiment 2 'tI $\sim>f I$ ) $m$ we next addressed what serves as the information for the affordance for

throwing? Previous studies have consistently shoun that the optimal weights

selected for throwing co-varied with object sizes in a function corresponding to

that of the classic size-ueight illusion, in which objects of increasing size must

weigh more to be perceived of equal he, aviness. It is possible that preferred

weights at each different size are perceived as equally heavy. Method The same objects uere used. Tuelve skilled adult throuers were recruited

to perform two hefting judgment tests. They were first asked to select optimal

objects at each size for maximum distance throus, and then they were asked to

select objects at each size that were felt equally heavy to a comparison object.

Two sizes of comparison objects were used (I-inch and 6-inch). Participants

were randomly divided into two groups' to perform the judgments. In each

group, the comparison object used was actually the one selected by the same

participant before as optimal for throuing, but this was not known by the par

ticipant. $300.00280 .00-J s \sim 200.00-<$ at $j i$

$150.00 \ldots$... lit o s:. U: 100.00: I S0.00"

๑. 90.JUDGMENT N'0\% 'Of'lhrotring .... \&:auaI Heaviness 
Nford.nce for lhrowing "Hem ... ss $1^{\sim} / /^{\infty} / \theta^{\prime} /$

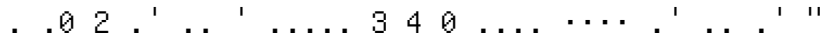

, ' 5 object Diameter (Inch) 6 Figure 2 Mean chosen

weights from both comparison groups as a function of

object diameter and judgment task. Filled squares connected

with a solid line represent the judgments of optimal

weights for throwing; unfilled squares connected with a

dashed line represent the judgments of objects that were

felt equally heavy to the given comparison object.

Results and Discussion As shown in Figure 2, there was no

significant difference between two

judgments in both groups, indicating that the objects

selected to be optimal for

throwing were also felt equally heavy to all throuers.

chosen weights increased

as object became larger, suggesting that both judgments were subject to the size

weight illusion. Conclusion The information about the affordance for maximum distance throus is dis

tinguished through learning to throw. It is based on the perceived heaviness.

People learn to detect the heaviness that is optimal for long distance throus.

Vision was required to allow the perceiver to identify the optimal value of

heaviness for throwing. Once the optimal heaviness is identified, the affordance

can be perceived, and the perception of the affordance can be generalized to any

Bingham, G., Schmidt, R., \& Rosenblum, L. (1989). Hefting for a maximum distance throw: A smart perceptual mechanism. Journal of Experimental Psychology: Human Perception and Performance, 15 (3), 507-528.

Busemeyer, J. R., \& Tounsend, J. T. (1993). Decision Field Theory: A dynamic cognitive approach to decision making. Psychological Review, 100,432-459.

Gibson, J. J. (1986). The ecological approach to visual perception. Hillsdale, N.T: Laurence Erlbaum Associates. 
Runeson, s. (1977). On the possibility of "smart"

perceptual mechanisms. Scandinavia Journal of Psychology, $18,172-179$.

Zhu, Q., \& Bingham, G.P. (2008). Is hefting to perceive the affordance for throwing a smart perceptual mechanism? Journal of Experimental Psychology: Human Perception and Pt!rformance, 34 (4), 929-943. 


\section{Chapter 5: Posture}

Bardy, B. G., Marin, L., Stoffregen, T. A., \& Bootsma, R. J. (1999). Postural coordination modes considered as emergent phenomena. Journal of Experimental Psychology: Human Perception and Performance, 25, 1284-1301.

Bonnet, Y., Fraisse, P., Lagarde, J., Ramdani, N., Ramdani, S. \& Bardy, B. G. (2007). Modelling of the human postural coordination to improve the humanoid control of balance. leE Int. Con! on Humanoid Robots.

Bonnet, Y., Fraisse, P., Lagarde, J., Ramdani, N., Ramdani, S. \& Bardy, B. G. (2008). Modelling postural coordination dynamics using a closed-loop controller. lEE Int. Corif. on Humanoid Robots, 61-66.

Kelso, J (1995). Dynamics patterns the self-organization of brain and behavior. The MIT Press. 2009 Taylor \& Francis Group, LLC Postural Stabilization of Visual Performance at Sea Fu-Chen Chen!, Yauen Yu!, Sebastien Villard 2,8 Thomas A. Stoffregen! luniversity of Minnesota, zuniversity of Montpellier-1, France classically, theory and research relating to the control of stance have fo

cused on the avoidance of falling, that is, maintaining the body's center of mass

over the base of support. Postural control-certainly helps to avoid falling, but it

may have other functions, as well. Postural actions move the center of mass, but

they also alter the position and motion of the head and, consequent ly, of the

eyes. Postural stabilization of vision has been demonstrated in a variety of con

texts; children (Jordan et aI., 2008) and the elderly

(Prado et aI., 2007), eye

movements (Stoffregen et aI., 2007) and fixation

(Stoffregen et aI., 1999), read

ing (Stoffregen et aI., 2000) and so on. In most previous cases have involved 
unperturbed stance, such that postural motion arose entirely from the person. In

some cases, standing participants have been exposed to optical motion, as for

example a moving room (Stoffregen et at 2006). In this study, we examined

postural stabilization of vision in a situation involving motion of the surface of

support. We studied experienced maritime creumembers on a ship at sea. Relations

between body motion and the performance of visual tasks are of increasing im

portance in naval applications, as work becomes increasingly focused on com

puter interaction. While at sea, standing participants performed visual tasks.

We varied the degree of ocular stability required for visual performance. Fol

lowing Stoffregen et aI. (2000), we predicted that sway would be reduced during

performance of a visually demanding task, relative to stance during performance

ofa task having reduced visual demand. We also varied stance width, that is, the

distance between the feet during stance. Hider stance is associated with reduc

tions in the magnitude of spontaneous postural sway (e.g., Day et aI., 1993), but

this relationship has not been evaluated in the context of continuous motion of

the support surface (cf. Henry et aI., 2001). We predicted that the magnitude of

sway would be negatively related to stance width. Previous studies of standing 
suay in moving environments have been limited to ship

simulators, and have

not manipulated stance width or visual task demand (e.g., Dobie et aI., 2003;

Nawayseh \& Griffin, 2006). Method The study was conducted aboard the $R / V$ At lantis, on the 5 th day of a 6 -day

cruise from San Diego to Mazatlan, Mexico. The At lantis was $84 \mathrm{~m}$ long and

displaced 3500 tons, and cruised at 12 knots. Ten creumembers participated on

a volunteer basis. Crew ranged in age from 25 to 54 years, and had from 1 to 28

years experience working at sea. Postural data were collected using a force plate (AMTI, Watertoun, MA)

sampled at $40 \mathrm{~Hz}$. Visual tasks and targets were the same as those used by stof

fregen et al. (2000). The study was conducted in the ship's Main Laboratory, a large room lo

cated amidships on the main deck. The force plate was positioned near a wall,

and visual targets were affixed to the wall with velcro. Targets were adjusted to

be at eye height for each participant. The visual targets consisted of sheets of

white paper $13.5 \mathrm{~cm} \times 17 \mathrm{~cm}$ and mounted on rigid cardboard. For the Inspec

tion task, the paper was blank. For the search task, the sheet was printed with

English text in a 12 point sans serif font. When on the force plate, participants

were facing athwartship (rather than fore and aft), such that roll motion of the

ship would be corrected by suay in the body's AP axis, and 
ship would be corrected by sway in the body's ML axis. Participants stood on the force plate with their heels on a line that was $1.0 \mathrm{~m}$

from the visual target. Lateral foot placement was determined by three pairs of

lines on the plate, such that the heels were $5 \mathrm{~cm}, 17 \mathrm{~cm}$ or $30 \mathrm{~cm}$ apart and the

angle between the feet was $10^{\circ}$. In the Inspection task, the target consisted of a

blank sheet of white paper; participants were instructed to keep their gaze within

the borders of the target. In the search task the target was one of 4 blocks of

text, each consisting of 13 or 14 lines of text. Prior to each trial the participant

was given a target letter ( $A, R, N$, or $S$ ), and asked to count the number of times

the target letter appeared in the block of text. At the end of each search trial, the

participant reported the number of letters counted, and their position in the text

at the end of the trial. During testing, the sea state was 2 on the Beaufort scale

(Beer, 1997). We used a $2 \times 3$ design with one trial per participant in each of six condi

tions. Each trial was $65 \mathrm{~s}$ long. We assessed postural activity in terms of the po

sitional variability of the center of pressure (CoP). Results and Discussion the results are summarized in

Figure 1. Analysis of variance revealed a

significant main effect of stance width, $F(], 8)=33.64, \mathrm{P}$ $<.001$, for movement in

the body's ML axis (Figure $1 A$ ). This effect is similar to 
tions of an inverse relation between stance width and the magnitude of $M L$ suay

(e.g., Day et aI., 1993). We also found 'a main effect of visual task, $F(J, 9)=$

12.35, $\mathrm{P}=.007$, for movement in the body's AP axis (Figure 1B). When per

forming the Search task, the magnitude of suay was reduced, related to suay during the Inspection task. This effect replicates a finding of stoffregen et al.

(2000). None of the participants reported seasickness at any point during the

cruise. ${ }^{\sim} 0 . g 0.7 \mathrm{r}-\mathrm{I}: . \mathrm{s}^{\sim} 0.6 \mathrm{rL}>-.0 .5 \mathrm{I}$

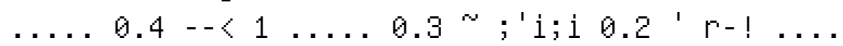

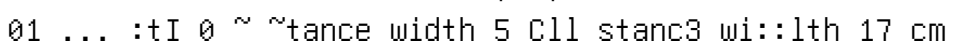

stance width $30 \mathrm{~cm},-, 2.5 \sim 02$ () 2 . $1.5 \mathrm{r} 1 \mathrm{r}$

l. $\sim . . t, \ldots l:: \mathrm{J}^{\sim} 0.5 .{ }^{\sim} \mathrm{h} . . . \cdots, \ldots>0$

Inspecti on task' Search ta sk Figure 1. Mean

positional variability of the CoP. Body's ML axis (Top).

Body's

$\mathrm{AP}$ axis (Bottom). We replicated the classical finding that the magnitude of postural sway is

inversely related to the distance between the feet in side-by-side stance (e.g.,

Day et aI., 1993), and we showed that this effect occurs on a ship at sea. This

finding suggests that variation in stance width might be used by sailors, on a

task-specific basis, to control the magnitude of suay at sea. Appropriate varia

tions in stance width would be related to the nature of the visual task at hand, to

the sea state (i.e., wave height), and to the ship's heading relative to the wind.

Complex and highly variable relations between these factors suggest that learn 
ing could be slow, which is compatible with anecdotal reports that it often takes

several days to get one 's "sea legs". We also replicated the finding of stoffregen et al. (2000) that the magnitude

of suay uas reduced during a reading-like task, relative to sway during a less

demanding visual task, and we extended this fmding to nautical conditions. In

our study, experienced sailors modulated their stance in ways that would tend to

maximize both visual performance and the efficiency of postural control. Future

research should examine how this skill is acquired, and whether the relation be

Beer, T. (1997). Environmental oceanography. Boca Raton, FL: CRC Press.

Day, B.L., Steiger, M.J., Thompson, P.D., \& Marsen, C.D. (1993). Effects of vision and stance width on human body motion when standing: implications for afferent control of lateral suay. Journal of Physiology, 469,479--499.

Dobie, T. G., May, J. G., \& Flanagan, M. (2003). The influence of visual reference on stance and walking on a moving surface. Aviation, Space, and Environmental Medicine, 74,838-845.

Henry, S. M., Fung, J., \& Horak, F. B. (2001). Effect of stance width on multidirectional postural responses. Journal of Physiology, 85, 559-570.

Jordan, A, Wade, M. G., \& Yoshida, K. (2008, June). Postural support for visual performance in children at risk for developmental coordination disorder. Talk given at NASPSPA 2008, Niagara Falls, Canada.

Nawayseh, N., \& Griffin, M. J. (2006). Effect of frequency, magnitude and direction of translational and rotational oscillation on the postural stability of standing people. Journal of Sound and Vibration, 298, $725-754$ 
Prado, J. M., Stoffregen, T. A, \& Duarte, M. (2007). Postural suay during dual tasks in young and elderly adults. Gerontology, 53,274-281.

Stoffregen, T. A, Bardy, B. G., Bonnet, C. T., Hove, P., \& Dullier, 0. (2007). Postural suay and the frequency of horizontal eye movements. Motor Control, 11, 86-102.

Stoffregen, T. A, Hove, P., Schmit, J., \& Bardy, B. G. (2006). Voluntary and involuntary postural responses to imposed optic flow. Motor Control, 10, 24-33.

Stoffregen, T. A, Pagulayan, R. J., Bardy, B. G., \& Hettinger, L. J. (2000). Modulating postural control to facilitate visual performance. Human Movement science, $19,203-220$.

Stoffregen, T. A, Smart, L. J., Bardy, B. G., \& Pagulayan, R. J. (1999). Postural stabilization of looking. Journal of Experimental Psychology: Human Perception \& Performance, 25, 1641-1658. Acknowledgements. We thank Captain A D. Colburn and Liz Caporelli of

the Woods Hole Dceanographic Institution, who made this study possible. 2009 Taylor \& Francis Group, LLC Immediate Awareness of the surrounding Environment in the Form of optical Pushes Makoto Inagami \& Ryuzo ohno Department of Built Environment, Tokyo Institute of Technology, Japan Gordon Cullen (1971), a keen urban designer, described the experience of

walking through tounscapes as "a journey through pressures and vacuums, a sequence of exposures and enclosures" (p.I0). During daily locomotion, humans

are constant ly auare of the environment "hat surrounds their bodies. They im

mediately feel the presence of surrounding surfaces, rather than intellectually

understand the geometrical layout. This article deals with such immediate envi

ronmental auareness, which was aptly described as "pressure" by cullen.

We have thus far attempted to investigate environmental awareness by an origi 
nal method (e.g., Inagami \& 0hno, 2008; Inagami, 0hno, \& Tsujiuchi, 2008).

Dur method uses the "feeling of pressure" ("appaku-kan" in Japanese) caused by

the surrounding environment. It is defmed as a feeling that changes in accor

dance with the extent to which the point of observation is spatially enclosed. The

feeling is similar to what has been studied as subjective enclosure or closeness

in environmental psychology (e.g., Stamps \& Smith, 2002). In our experiments,

participants continuously rate their feelings while walking through a daily envi

ronment. Our method analyzes the relationship between the participants' ratings

and several environmental measurements (e.g., visible area of buildings) to cap

ture their awareness of the surroundings. This article analyzes the data obtained in the experiment reported by Ina

gami et al. (2008) through another method. On the basis of the result, we discuss

the relationship between environmental awareness and a phenomenon called

"optical push" (e.g., Shaw \& Kinsella-Shaw, 2007). Method The experiment was conducted using a 425 -meter outdoor route in our uni

versity campus. As shown in Figure 1, the route contained various characteristic

places such as a tunnel, a hill, and areas surrounded by tall buildings. Fourteen

graduate students (seven males and seven females) individually participated in 
the experiment. They continuously rated their feelings of pressure while walking 139

along the route. Their ratings were outputted by turning a dial on a portable rat

ing device. Since the data were recorded in time series, we converted them to

850 pieces of data in such a way that each piece

approximately corresponded to

850 points at 0.5 -meter intervals along the route. In addition, the converted data

were standardized for every participant and then averaged across all participants. Figure 1. Experimental route in our university campus. To measure the changes in the environmental layout along the route, we

used our original computer program (for details see ohno, 1991). The measure

ment was based on the environmental data (site plan, land configuration, and

tree data) created with computer-aided design (CAD)

software. We set 850

points of observation at 0.5 -meter intervals along the route, which were at the

level of 1.5 meters above the ground. At every point, the program radiated 1944

scanning lines all around (72 horizontally $\times 27$

vertically) and recorded the dis

tances to the surrounding environmental surfaces. The measurement was limited

to within a 72 -meter radius of each observing point. As a variable to describe the environmental layout, we adopted the averages

of the measured distances. According to ecological optics (Gibson, 1979), envi

ronmental information is conveyed by the global flow of 
the ambient optic ar

ray. The optic flow radially expands from the direction of locomotion and con

tracts toward the opposite direction. The flow velocity of each environmental

component not only varies with the angular deviation from the focus of expan

sion, but is also inversely proportional to the distance from the observing point.

In view of the optical properties, we divided the surrounding environment into

three parts with respect to the angular deviation front $\left[0-60^{\circ}\right]$, middle $[60$

$\left.120^{\circ}\right]$, and back $\left[120-180^{\circ}\right)$ ) and calculated the average distances to the envi

ronmental surfaces for each part. Q) ${ }^{\sim} \ldots$ Q $):$ : J ... til 8 til til Q) I ....N a.. ${ }^{\circ} \theta \mathrm{Cl} . f$; Qi Q) $l \mathrm{~L}$. $30-30100200$ Experimental Route (m) 300 observed value 400 Figure 2. Prediction of the feeling of pressure along the route. The observed values

are the mean ratings for all participants ( $N=14)$. Results and Discussion To analyze the relationship between the participants' ratings and environ

mental variables, we performed a multiple regression analysis using the following model: (1)

where $\mathrm{Fp}$ is the feeling of pressure and $\mathrm{Dr}, \mathrm{D} \mathrm{m}$, and $\mathrm{Db}$ are the average distances

to the front, middle, and back parts of the surrounding environment, respec

tively. The parameters B $\theta, \mathrm{B}, \mathrm{j}, \mathrm{B} 2$, and B 3 were estimated to be $3.097, .085, .002$, and .048 , respectively ( $p<.01$ in all cases). Figure 2 illustrates the varia

tions in the observed (i.e., rated) and predicted values along the route. The resul

tant coefficient of determination is .88, indicating that 
the participants' ratings

have a high correlation with the variables of the environmental layout. This result suggests that the participants continuously felt pressure from the

surrounding environment while proceeding along the route. Their ratings are

considered as the integration of the pressures caused by each of the surrounding

surfaces. During the rating task, the constantly changing feeling was outputted

directly by the rating device. The rating was conducted intuitively without the

intervention of any intellectual process such as the estimation of the size of the

surrounding surfaces. Viewed in this light, the feeling of pressure is environ

mental awareness itself rather than something that derives from it. In other

words, humans are immediately aware of the surrounding environment in the

form of the feeling of pressure caused by the surfaces. We speculate that the feeling of pressure could be interpreted as the so

called "optical push." As an example of the phenomenon, Shaw and Kinsella

Shaw (2007) cited an error that pilots sometimes make while controlling low

flying airplanes. Even if the pilots intend to keep a

fixed altitude, they involun

tarily change the altitude when crossing the boundary between sea and land. The

abrupt change in the density of texture induces a virtual

force. The pilots gener

ate an action as if being pushed by the optical flow. In 
daily locomotion such as in our experiment, the same sort of force potentially 141

acts on the perceivers. They are pushed by the global flow of the ambient optic

array and thereby continuously feel varying degree of pressure touard the envi

ronment surrounding their bodies. The pressure moderately guides, rather than

forcibly controls, their locomotion through the environment. Given this perspec

tive, environmental awareness could be conceptualized not as mental representa

Cullen, G. (1971). The concise tounscape. London: Architectural Press.

Gibson, 1. 1. (1979). The ecological approach to visual perception. Boston: Houghton Mifflin Company.

Inagami, M. \& Dhno, R. (2008). Anisotropy of environmental auareness caused by spatial changes during locomotion. In C. Holscher (Ed.), Spatial Cognition 2008: Poster Presentations (pp.97-100). BremenlFreiburg: SFB/TR 8 Spatial Cognition.

Inagami, M., ohno, R., \& Tsujiuchi, R. (2008). Phenomenal auareness of the surrounding space: An ecological perspective. Cognitive Studies: Bulletin of the Japanese Cognitive science society, 15 (1), 134-143.

ohno, R. (1991). Ambient vision of the environmental perception: Describing ambient visual information. In J. Urbina-Soria, P. Ortega-Andeane, \& R. Bechtel (Eds.), Healthy environments: Proceedings of the 22nd annual conference of the Environmental Design Research Association (pp. 237-245). Oklahoma City: Environmental Design Research Association.

Shaw, R. \& Kinsella-Shaw, 1. (2007). Could optical 'pushes' be inertial forces? A geometro-dynamical hypothesis. Ecological Psychology, 19 (3), 305320.

Stamps, A. E. \& Smith, S. G. (2002). Environmental 
enclosure in urban areas, Environment and Behavior, 34 (6), 781-794. 2009 Taylor \& Francis Group, LLC Postural Response to a Suprapostural Task in Children at Risk for Developmental Coordination Disorder Azizah Jor'dan, Michael Wade, Ken Yoshida, \& Thomas A. 'Stoffregen University of Minnesota, Minneapolis, MN Developmentally, postural stability is a critical achievement as a precursor to locomotion. Posture is not static and the exhortation to "stand still" is

not possible. Standing quietly the human body oscillates at approximately $2 \mathrm{~Hz}$. Recording postural motion (suay) is a variable which can be investigated as a mediator of attentional effort. This study investigated hou posture (a biomechanical variable that mediates coordination dynamics) is relevant to the study of movement problems in children at risk for Developmental Coordination Disorder (DCD). We proposed an experiment to determine changes in postural suay as a function of engaging in a suprapostural task requiring cognitive effort. Method children at a local Elementary school volunteered to participate in the study. The children ranged in age from 8 yrs 9 months to 11 years (.? mean age

$=10$ years, SD 0.72). Participants were assessed using the Movement $\mathrm{ABC}$

(Henderson \& Sugden, 1992). Six children scored at or below the 15 th percen

tile in the Movement $\mathrm{ABC}$ and were considered at risk for DCD. Five children

scored well above the 15 th percentile and were designated as a typically developing (TDC) control group. Participants were asked to stand behind a designated mark 1 meter from

a target. The visual task comprised two conditions; one a blank target task (BT)

and the other a counting task (CT). The BT target was a plain white poster

board in which participants could look anywhere within its perimeters. The cT

target comprised of a stream of printed letters of the alphabet in random order. 
Participants were instructed to count the frequency of a designated letter during

each trial. At the end of each trial, participants reported where they were in the

text stream and the total number of target letters counted. We monitored move

ment of the head and torso. 143 For the CT condition, visual perfonnance was assessed as percent accuracy

(ratio of the number of letters reported to the total number present in the dis

play). A one-sample Kolmogorov-Smimov Test (K-S) analyzed the distribution

of errors to detennine any differences among the sample and the expected distri

bution Results and Discussion Visual performance. The TDC and the DCD group mean percentage cor

rect scores was $89.1 \%$ and $83.3 \%$, respectively. The small group difference in

accuracy scores may be a consequence of too Iowa level of task difficulty,

which likely produced a ceiling effect. A more difficult or different type of task

may remove the ceiling effect and increase the sensitivity to detect possible dif

ferences in postural motion between the two groups. Fieure h: Vribility of Position 1.2 .10 .850 .6 $0.40 .20^{\sim}$ Fieufe Ie: 80 of Velocity $0.8 \ldots 0.6$ $r^{\sim} 0.45$. TOC $0.2 \sim 0$ OCO Fieure Ib: Mean Velocity

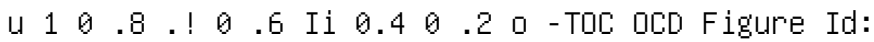
$\mathrm{P}^{*}$ th Length $2.521 .5 \mathrm{Ii}$.TOC .1 0.5. OCO 0 ST CT Figure 1. Summary of torso data. A. Variability of position. B. Mean velocity. C.

Standard deviation of velocity. 0. Path length. AP: Anterior-posterior. ML: Mediola

teral. BT: Blank task. CT: Counting task. ToC: Typically developing children. oco: 
Children at risk for developmental coordination. disorder. Postural activity. For movement of the head, there were no significant ef

fects. Data for torso motion are summarized in Figure 1. We found significant

main effects of Task on the mean velocity of torso motion in ML $(p=.027)$, and

for the standard deviation of velocity of torso motion in the ML axis $(p=.012)$. We sought to generate further support for the hypothesis that children at

risk for DCD show different postural response characteristics when engaged in a

more demanding suprapostural task. This would provided further support for

data from our laboratory (Johnson \& Wade, 2007); thus adding to the proposi

tion that DCD was a perceptual motor deficit characterized by a diminished per

ception/action coupling when a motor response (posture) was linked to a task

outcome that required increased attention and cognitive effort. The significant task effect across groups was present for the mean velocity

and the standard deviation of velocity. These fmdings were consistent with

Stoffregen et al. (1999; 2000) in adults and Prado, Stoffregen, and Duarte (2007)

in the elderly, confirming that postural stabilization of visual performance oc

curs at least as early as the age of children in our study. We did not fmd differ

ences between the $\mathrm{DCD}$ and TDC groups. It may be that $\mathrm{DCD}$ does not alter the

use of body sway in the service of visual performance. 
differences might be obtained in new studies using a greater number of partici

Henderson, S.E., \& Sugden, D.A (1992). Movement assessment battery for children. London: The Psychological corporation.

Johnson, D., \& Wade, M. (2007). Judgment of action capabilities in children at risk for developmental coordination disorder. Disability and Rehabilitation, $29,33-45$.

Prado, 1., Stoffregen, T., \& Duarte, M. (2007). Postural suay during dual task in young and elderly adults. Gerontology, 53,274-281

Stoffregen, T. A, Pagulayan, R. 1., Bardy, B. G., \& Hettinger, L. J. (2000). Modulating postural control to facilitate visual performance. Human Movement science, $19,203-220$.

Stoffregen, T. A, Smart, L. J., Bardy, B. G., \& Pagulayan, R. J. (1999). Postural stabilization of looking. Journal of Experimental Psychology: HumanPerception and Performance, $25,1641-1658$.

Wade, M., Lindquist, R., Treat-Jacobson, D., \& Taylor J. (1995) Dptical flow, spatial orientation, and the control of posture in the elderly. Journal of Gerontology, 50, $51-58$.

(6) 2009 Taylor \& Francis Group, LLC Consequence of Internally Versus Externally Generated Visual Motion on. Postural Regulation in a Virtual Environment: The Importance of Perception and Action Rather than Perception and Reaction Eric Littman, Edward W. Otten \& L. James Smart, Jr. Miami University, oxford oH In order to achieve most goals, one must be able to successfully assess and

interact with one's surroundings. These behaviors, in turn, rely on the ability to

recognize the affordances that exist within a given context. Successful coordina

tion of these activities allow for people to behave in a manner that is functional 
and reduces the distance between the current and goal

states. These activities

can be described as being future-oriented or prospective (literally, forward

looking; E.1. Gibson, 1969; Reed, 1996; Gibson \& Pick, 2000) in nature. In the

performance of motor tasks, the first step involves the basic control and coordi

nation of body segments (posture; cf. Smart \& smith, 2001). When this is

achieved the person is then in a position to perform subsequent actions. In our everyday experience there exists a clear relationship between what we

perceive and the actions that we produce (perception-action cycle; Gibson,

1986). This type of relation can be characterized as closed-loop in nature (where

the persons actions influence and are influenced by information from the world).

However, there are a number of situations where the relation between perception

and action is open-looped (where the person's actions have little or no conse

quence on the information generated by the environment). In both cases the goal

of the person is to interact successfully with the surrounding environment; it is

the manner in which these interactions are accomplished that differs as a func

tion of the type of control (internal, closed-loop or external, open-loop). Problems can arise when an inappropriate strategy is implemented by the

person; one such problem in motion venues is the occurrence of motion sick 
ness. Through research and innovation engineers have produced better vehicles,

boats, and planes that suppress the motion characteristics that make people mo

tion sick. While the virtual reality world expands, better technology has pro

duced more motion sickness. Sensory conflict theory is probably the most well known and intuitive theory.

The theory asserts that motion sickness is produced when the brain receives in

formation from different senses unsynchronized with each other (reporting dif

ferent motion 'realities'). While the brain attempts to resolve this conflict, one

can become motion sick (Reason, 1975; Dman, 1982). Despite its intuitive ap

peal, Conflict Theory does not provide an objective measure of conflict, nor

does it allow sickness to be predicted a priori. Postural Instability Theory, on

the other hand, asserts that motion sickness is not related to a sensory conflict,

but instead to a decreased ability to appropriately control one's postural motion

(Riccio \& Stoffregen, 1991). The longer one remains unstable, the more likely

that sickness will occur. Research has shown that postural motion can predict

sickness (Stoffregen \& Smart, 1998; Stoffregen, et aI, 2000; Smart, stoffregen,

8 Bardy, 2002). The specific goal of the study is to determine the role of control on the occur

rence of motion sickness, and the ability to make 
prospective adjustments (ac

tions that serve a future goal). Research has indicated that these factors can be influenced by perceived or actual control (Rolnick \& Lubow, 1991). The study

entailed the measurement and subsequent analysis of seated participants' postur

al motion while immersed in a virtual environment. It is believed that disrup

tions in postural control that can be produced by YEs may lead to adverse side

effects (cf. Riccio \& Stoffregen, 1991) and that these effects will be more pro

nounced when the person is exposed to externally generated (open-loop) motion. Method Tuelve undergraduate students ranging in age from 1822 years. All par

ticipants had normal or corrected-to-normal vision and were in good health with

no history of vestibular dysfunction or dizziness/falls. Participants received class

credit for their participation. Participants were aware that there was a chance

that they could become motion sick. Motion Tracker: body movement was tracked using a magnetic tracking

system (Flock of Birds, Ascension, Inc.) Four sensors were used. Motion was

sampled at $40 \mathrm{~Hz}$. Game console/softuare: A Nintendo 64 video game system,

with standard controller, was used to generate the stimuli for the study. A first

person shooter (Goldeneye; Rare, Inc.) was used in this study. Projector: A

Sharp PG-C30XU projector was used to present the stimuli, producing an image

with an unobstructed visual angle of $29^{\circ}$ (U) $\times 37^{\circ}$ 
(H)-distance $2.76 \mathrm{~m}$. Participants were told the nature of the study and were asked to fill out a

consent form, demographics sheet, and a simulator sickness Duestionnaire

(SSQ, Kennedy, et aI., 1993). Participants were asked to perform two balance

checks before beginning the experiment (these were repeated before participant

was allowed to leave). Participants sat on a stool, holding the game controller

comfortably in their hands. Participants ${ }^{*}$ each session were presented with

three types of trials: 3 baseline (one post, 20 sec), 5 control (one post, 60 sec),

and 6 experimental ( $300 \mathrm{sec}$ ). Three of the experimental trials used internal con

trol (the participants were playing) and three of the trials used external control

(the experimenters were playing). This is denoted by the terms active and pas

sive, respectively. Results and Discussion Dispersional Analysis. The basis of the dispersional analysis is to detennine

the spread of the probability density function at the highest level of resolution,

then to lump nearest-neighbor elements of the signal together to obtain the local

mean of the pair of elements. The dispersion is then calculated at this reduced

level of resolution, and repeated with successively larger groupings of near

neighbors. The slope of the log-log relationship between dispersion and level of

resolution gives the fractal dimension $D$. A value of $D$ near 


\section{5 is indicative of}

random uncorrelated noise, while a value near I is

indicative of unifonnity over

all scale lengths (Bassingthuaighte, 1994).

Anterior-Posterior: A 2 (sick vs. well) by 2 (active vs.

passive) mixed mod

el ANOV A resulted in a significant main effect of control

$(\mathrm{F}(\mathrm{I}, 12)=9.853, \mathrm{P}=$

.009), indicating that the value of the dispersional

exponent was smaller when

the participants were in control of the environment ( $M=$ $1.047, \mathrm{SD}=.038$ ) than

when they were not in control $(M=1.096, \mathrm{SD}=.067)$. There was no main ef

fect of the condition of the participant (sick vs. well) and there was no interac

tion between the relative control of the participant and the condition of the par

ticipant. Participants who became sick tended to exhibit more antipersistent correc

tive) motion than participants who remained well. The results suggest that those

participants who remained well were able to find an appropriate stable postural

strategy while those participants who became sick were searching for such as

Bassingthwaighte, 1. B., Liebovitch, L. S., Bruce, 1. W. (1994). Fractal Physiology. New York, NY: 0xford University Press, Inc. Gibson, EJ. (1969). Principles of Perceptual Learning and Development. East Norualk, CT: Appleton-Century crofts.

Gibson, J. 1. (1986). The Ecological Approach to Visual Perception. Hillsdale, N.J: Laurence Erlbaum Associates. Gibson, E.J., \& Pick, AD. (2000). An Ecological Approach to Perceptual Learning and Development. New York: Oxford University Press. 
Kennedy, R.S., Lane, N.E., Berbaum, K.S., \& Lilienthal, M.G. (1993). Simulator sickness questionnaire an: enhanced method for quantifying simulator sickness. Inter $J$ Aviation Psychology 3,203-220. Oman, C.M. (1982). A heuristic mathematical model for the dynamics of sensory conflict and motion sickness. Acta 0tolaryngol. 44 (suppl. 392), 144.

Reason, 1. T., and Brand, J. J. (1975). Motion Sickness. London: Academic Press.

Reed, E.S. (1996). Encountering the World: Touard an Ecological Psychology. New York: oxford University Press.

Riccio, G. E., \& Stoffregen, T.A. (1991). An ecological theory of motion sickness and postural instability. Ecological Psychology, 3, 195-240.

Rolnick, A, \& Lubou, R. E. (1991). Why is the driver rarely motion sick? The role of controllability in motion sickness. Ergonomics, 34 (7), 867879.

Smart, L. J., \& Smith, D. L. (2001). Postural dynamics: Clinical and empirical implications. Journal of Manipulative and Physiological Therapeutics, 24 (5), 340 -349 .

Smart, L. J., Stoffregen, T. A, \& Bardy, B. G. (2002). Visually induced motion sickness predicted by postural instability. Human Factors, 44 (3),451-465.

Stoffregen, T. A, Hettinger, L. R., Haas, M. W., Roe, M., \& Smart, L. J. (2000). Postural instability and motion sickness in a fixed-base flight simulator. Human Factors, $42(3), 458-469$.

Stoffregen, T. A \& Smart, L. J. (1998). Postural instability precedes motion sickness. Brain Research Bulletin, 47 (5), 437-448.

(c) 2009 Taylor \& Francis Group, LLC The Effect of Dpen vs. Closed-Loop optic Flow on Visually Induced Motion Sickness Edward W. Otten \& L. James Smart, Jr. Miami University, oxford OH Eighteen years ago Riccio and Stoffregen (1991) asserted that motion sick

ness was produced by disruptions in action (postural instability) rather than defi 
ciencies in perceptual processing (sensory conflict theory; Reason \& Brand, 1975; Oman, 1990). Since then a number of studies have demonstrated that ma

nipulating perceptual information (optic flow) can produce these behavioral dis

ruptions and subsequent motion sickness both in natural and virtual settings

(Stoffregen \& Smart, 1998; Stoffregen, Hettinger, Haas, Roe, \& Smart, 2000; Smart, Stoffregen, \& Bardy, 2002;

Smart, Dtten, \& Stoffregen, 2007; Villard,

Flanagan, Albenese, \& Stoffregen, 2008). These studies all utilized a similar

mechanism as participants in all studies were exposed to motion that closely

resembled that which uould be produced by their oun postural suay. Riccio and

Stoffregen (1991) suggested that using this type of motion could be especially

nauseogenic. Stoffregen and Smart (1998) attributed this potential outcome to

the phenomenon of wave interference, which states that when similar wave

forms come in contact with each other, the waves are disrupted; the greater the

similarity between waveforms, the more catastrophic the result of the contact.

We suggest that the behavioral consequence of this type of interference is that

the participant will no longer be able to use the perceptual information coptic

flow) obtained to effectively regulate his or her behavior. The second similarity among these studies is that the motion produced uas

created artificially (computer-generated) and independent of the motion gener 
ated by the participant. While this type of stimuli in many ways represents fa

miliar situations (movies, television, video gaming), it doesn't allow for direct

testing of some of the predictions made by Riccio and Stoffregen (1991), nor

does it allow for the direct testing of the importance of the nature/existence of

perception-action coupling. The current study sought to address these issues by

exposing participants to their oun motion, in a non-coupled (playback), normal

coupled (real-time), or reverse-coupled (real-time motion phase shifted $180^{\circ}$ )

manner. It was expected that participants in the normal coupled condition would

be more stable than the participants in either the reversed coupled condition or

the uncoupled condition, and that sick participants would be more unstable than

well participants in each condition. Method Participants. Thirty participants (about $40 \%$ female with a mean age of 19.5

yrs) were recruited trom the Psychology department participant pool. Ten par

ticipants were used in each condition. Thirty-seven percent (378) reported being motion sick in the past, $46 \%$ reported no prior motion sickness, and $17 \%$

reported that they were not sure. All participants were in their normal state of

health and had normal or corrected to normal vision. Materials. One computer was used in this study to both display the stimulus

through a head-mounted display (HMD; i ${ }^{\sim 0}$ Display systems 
i-glasses, Menlo Park, CA), and record the motion of the participants. The HMD display had a

visual angle of 18.4 degrees vertically and horizontally. The motion of the par

ticipants was captured using a magnetic tracking system (Flock of Birds; Ascen

sion, Inc., Burlington, VT) and recorded using a softuare package called vizard.

The program can record motion in three axes of translation and three axes of

rotation. AP head motion was used to create the movement of the stimuli. The

stimulus consisted of a star field (a pattern of random white dots on a black

background the translated in the anterior-posterior (AP) direction). It was dis

played to simulate the "stars" at a distance $\cdot$ of $3.3 \mathrm{~m}$. The star field would change

trom white to red at quasi-random intervals during the experimental trials. The

same softuare package was used to create the star field stimuli and display it to

the participant. Procedure. The participants were asked to stand comfortably in the labora

tory while the researchers measured their postural suay. The experiment con

sisted of five 10 min trials. The first trial served to measure the baseline (without

computer-generated stimulus) suay of the participant. During this trial, the par

ticipant stood in the darkened lab 3 meters trom a single bright light at approxi

mately eye height. The participant wore a. set of welding goggles with the dark 
glass replaced with clear glass to approximate the field of view and weight of

theHMD. After the baseline trial, up to four experimental trials uere conducted

(depending on whether the participant became motion sick via self-report). In

the non-coupled (UC) condition, the motion of the star field was a playback of

the participant's baseline motion. In the normal-coupled (NC) condition, the

motion was generated in real-time by the participant. In the reverse-coupled

(RC) condition, the motion was generated in real-time by the participant, but

was phase shifted 1800 - In these trials the participant was exposed to the star

field stimulus and asked to remember how many times the star field changed

trom white to red (to insure that they were paying attention to the stimulus). At

the conclusion of each trial, the participant was asked 1) how many times the

stimulus changed trom red to white and 2) how the participant felt. Results and Discussion Motion sickness incidence: All three conditions produced motion sickness in participants, $40 \%$ in the UC condition, $30 \%$ in the NC condition, and $70 \%$ in the RC condition. As in previous studies, reports of motion sickness were unambiguous and corresponded with significant increases in 880 (Kennedy, Lane, Berbaum, \& Lilienthal, 1993) scores. Postural motion: Postural motion was analyzed using a fractal analysis called dispersion analysis (Bassingthuaighte, Liebovitch, \& West, 1994). Like other fractal analyses (e.g., DF A; Villard; et aI., 2008), this analysis measures the self-similarity of a waveform over time scale (i.e., whether smaller units of the waveform can predict the behaviour/structure of larger units of the waveform). The analysis was performed on each of the 
experimental trials and compared using linear contrasts (to test the specific predictions made)..$s \sim j 6 f \sim \sim 1$

$.161 .141 .121 .11 .08^{\circ} \operatorname{cs} i: 1<1.061 .041$ $.021 \mathrm{NC}$ RC UC Candltiln Figure 1. Mean (SE) dispersion values (range 11.5 ) as a function of condition and health, higher numbers indicate less stability ( $N=30)$. Note the difference between sick and well participants in the coupled conditions that is absent in the non-coupled condition. The analysis indicated that sick participants $(M=1.120, S D=.0593)$ had

significantly higher dispersion values than the well participants $(M=1.083, \mathrm{SD}$

$=.0486), t(86)=-2.92, P=.004$. The analysis indicated that participants in the

UC condition ( $M=1.103, \mathrm{SD}=.0537)$ did not have significantly different dis

persion values than participants in the $N C$ and $R C$ conditions $(M=1.101, \mathrm{SD}=$

$.0536), t(86)=.242, p=.809$. Finally, the analysis indicated that participants in

the coupled well condition $(M=1.079, S D=.0469)$ had significantly lower dis

persion values than participants in all other conditions $(M=1.106, S D=.0552)$,

$t(86)=-2.253, p=.027$ (Figure 1). Similar to previous studies postural instability preceded reports of motion

sickness. Participants who later become motion sick exhibited less fractal (self

are the positioning of the feet. In side-by-side stance, we control stance width,

that is, the distance between the feet. We also control stance angle, the angle

between the feet. In both clinical and exp.erimental research on stance, the val

ues of stance width and stance angle often are not controlled, and not analyzed. 
In other studies, these parameters of stance are fixed by the experimenter, rather

than being chosen by those standing. McIlroy and MaId (1997) took stance

width and stance angle as dependent variables, asking participants to stand qui

etly with their feet positioned comfortably. They found that self-selected stance

width tended to be approximately $17 \mathrm{~cm}$. Standing posture is of great importance at sea, and adjustments made to the

control of stance are an essential part of "getting one's sea legs". Research on

postural control as it relates to shipboard life has not utilized stance width or

stance angle as dependent variables (e.g., Dobie et aI., 2003; Nawayseh \& Grif

fin, 2006). We assessed self-selected stance width on two ships at sea. In one case, we

first measured stance on land, and compared it to subsequent measures made at

sea. In the other case, measurements taken at sea reflected changes in sea state

over several days. Method The study was conducted aboard the RIV Atlantis, on the 5 th day of a 6 -day

cruise from San Diego to Mazatlan, Mexico. The At lantis was $84 \mathrm{~m}$ long and

displaced 3500 tons, and cruised at 12 knots. The other ship was the RIV Tho

mas G. Thompson, during a 10-day cruise that began in Samoa and ended in

Hobart, Tasmania. The Thompson was $84 \mathrm{~m}$ long and displaced 3000 tons, and

cruised at 11 knots. In total, 20 creumembers (10on each ship) participated on 
a volunteer basis. Crew ranged in age from 25 to 60 years, and had from 1 to 30

years experience uorking at sea. Due to local conditions, procedure differed slightly on the two cruises. One

day before the beginning of the Atlantis cruise, we measured the preferred

stance of participants, using a nearby office provided for our use. At sea, we

measured preferred stance each day for the first five days. We were not able to

take terrestrial data prior to the Thompson cruise. Rather, we measured stance

each of the first four days at sea, and again on the 9 th day. At sea, stance was measured separately with each participant facing for

ward, and facing port. In each case, the Experimenter stood approximately $3 \mathrm{~m}$ in front of the participant and asked him or her to take three steps forward and

then stop. Using a tape measure, we measured the distance between the midline of the heels (stance width), and the distance between the great toes. The heel and toe data were used to compute stance angle. Results and Discussion On the RIV Atlantis (Figure 1), sea state never exceeded 2 on the Beaufort scale (Beer, 1997). Stance width on land $(19.0 \mathrm{~cm})$ was similar to the report of

McIlroy and Maki (1997). However, stance uidth at sea was greater than stance

width on land, both when facing laterally (mean = 23.5 $c m$ ), $t(58)=2.93, P=.005$, and when facing forward (mean $=23.8 \mathrm{~cm}), t(58)=3.14, P=.003$. At sea, stance width did not change over days. These effects indicate that experienced

mariners selected wider stance at sea than on land, even during very mild sea states. Wider stance tends to reduce the magnitude of standing body suay (e.g.,

Day et aI., 1993). Thus, it may be that experienced mariners use stance width as 
a means to modulate the magnitude of body suay. The lack of change over days

(at sea) may arise from the experience of the creumembers, from the consistent,

mild sea state, or both. . JLand 23 Day $t 4$ Figure

1. RIV At lantis, showing stance width when facing to port. 5 On RIV Thomas G. Thompson, sea state varied from 3 to 4 on Days 12 ,

from 2 to 3 on Days 34 , and was at 6 on Day 9. As Figure 2 shous, stance

width (when facing port) changed over days. For days 14 , the slope when

facing port was -0.73 , which differed from $0, p<.05$. The slope when facing

forward (0.045) did not differ from 0. 21 E 20.5 U $2 . \mathrm{J}$

.c: : : : $19.5=$ ii $19 \cdot \mathrm{i} 16$ ! i Q) $18 \mathrm{U} 17.5 \sim 1 \mathrm{ID}$

17 en 16.5 1B Day1 gay2 Da)G Day<! Dalg Figure 2.

R/V Thomas G. Thompson, showing stance width when facing to port. On RlV Thomas G. Thompson, stanc.e width when facing port was higher

than is typically observed on land (McIlroy \& Maki, 1997)

for the first day at

sea. Over the course of the first four days at sea, stance width narroued, and on

Day 4 it was similar to what has been observed on land. The narrowing was

consistent with the improving sea state over the first four days, as was the in

crease in stance width observed on Day 9 , when the highest sea state was ob

served. The absence of an effect for stance when facing foruard may indicate

that the majority of ship motion on Thompson was in the roll plane. We measured self-selected stance width on two ships at sea. We found dif 
ferences, across the two cruises, in the effect of a ship on stance width. With

consistent, mild sea states (on RIV Atlantis), stance width at sea was stable, and

was wider than in the same participants on land. With variable sea states, stance

width appeared to be strongly influenced by sea state. Dverall, the results sug

gest that stance width is flexible, and that people select stance width based on

local conditions. Our participants uere experienced mariners. It would be in

teresting to see whether similar effects would obtain in persons with no previous

experience at sea, and whether any adaptation in stance width would be related

Beer, T. (1997). Environmental oceanography . Boca Raton, FL: CRC Press.

Dobie, T. G. , May, J. G. , \& Flanagan, M. (2003). The influence of visual reference on stance and walking on a moving surface. Aviation, Space, and Environmental Medicine, 74,838-845 . McIlroy, W. E., \& MaId, B. E. (1997). Preferred placement of the feet during quiet stance: Development of a standardized foot placement for balance testing. Clinical Biomechanics, 12, 66-70.

Nawayseh, N., \& Griffin, M. J. (2006). Effect of frequency, magnitude and direction of translational and rotational oscillation on the postural stability of standing people. Journal o/Sound and Vibration, 298, $725-754$.

(c) 2009 Taylor \& Francis Group, LLC Infants' Visually Induced Postural suay: The Effect of the Attractiveness of Fixation Mark Aki Tsuruhara 1 , Yasunobu Katsumata 2 , Michiteru Kitazake, Akitoshi Hanazaua 3 , So Kanazawa 4 , \& Masami Yamaguchi 1 ,5 [Chuo University, 2Toyohashi University of Technology, lKyushu Institute of Technology, 4Japan Women's University, 5PRESTO, JST. When you are on a stationary train and the train next to yours is moving, 
you might feel that you are moving (vection), and you might lose your balance

(visually induced postural sway). It is welt known that visual stimuli affect pos

tural control and the perception of self-motion (see Howard 1982, for a review). Visually induced suay has been regarded as the result of the misperception

of self motion. More specifically, movement of large visual stimuli sometimes

cause observers to perceive that they are moving and that the large visual stimuli

are stationary. This misperception may cause a loss of balance. Some previous studies, however, suggested that visually induced suay may

not be due to misperception of self motion. Previc and his colleague showed that

induced sway could occur before the observer perceived vection (Previc, 1992;

Previc \& Mullen, 1991). Another explanation for visually induced suay is that observers try to fixate

their eyes on moving stimulus, and their body suays in accordance with the

moving of the stimulus (0ullier, Bardy, Stoffregen \& Bootsma, 2002). Thus, the

attention to the stimulus could affect visually induced suay in adults. In this study, we examined the development of the effect of attention on

visually induced suay. Developmental studies reported that visually induced

postural sway has been shown in children (e.g., Lee 8 Aronson, 1974; Lee 8

Lishman, 1977) and in adults. The effect of attention on suay in infants, how

ever, has not been clear. In our experimental trials, 
infants were presented with a

moving background and/or a fixation mai' $k$. The attractiveness of the fixation

mark was varied to control the attention of infants.

Methods Participants were 7-10-month old infants (N=12).

The participants were

screened based on the observation and confirmation by the parents that they

could sit up by themselves. Some of them could craul, but none of them could

walk by themselves. Infants sat at the front of the $152.4 \mathrm{~cm}$ (60 in.) screen (74.7 deg width $\times 59.5$

deg height in visual angle), on which the stimulus display was rear-projected

(Figure 1). The stimulus display was composed of a fixation mark and random

dots background. The viewing distance was maintained at approximately $80 \mathrm{~cm}$. Prior to each trial, a fixation mark turned on and off at the center of the

screen, accompanied by a brief sound. When the infant looked at the fixation

mark, the experimenter initiated the trial. During each trial, the fixation mark

and/or random dots (background) moved leftuard-rightuard or just remained

stationary for $2.5 \mathrm{~s}$. The motion speed was modulated in raised cosine and its

frequency was $0.2 \mathrm{~Hz}$, so that the movement started at the center of the screen

and went leftward or rightward and then came back in the opposite movement.

The maximum speed of the motion was $11.9 \mathrm{deg} / \mathrm{s}$ or 19.3 deg/s, but the results

did not differ significantly, so we combined them in 
analysis. The attractiveness of the fixation mark

(attractive character/ simple square),

the movement of the background (motion / no motion), and

the movement of the

fixation mark (motion / no motion) were varied, and all

combinations were pre

sented to each infant. The behavior of the infant was

videotaped throughout the

experiment. Only the infant's looking behavior was visible

in the video. One

observer (rater), who was unaware of the stimulus identity, judged whether the

infants looked at the screen, and whether the infants' gaze and posture changed

on each trial. Additionally, the movements of the infants were quantified and

judged by analyzing the video-image sequences through a computer image

analysis detecting the movement of body parts. Figure 1 . The experimental setting. Results and Discussion the ratios of the gaze and the postural movements were calculated for each infant in each condition by dividing the number of the trials in which the gaze or

posture of the infant moved by the total number of the trials in which the infant

looked at the screen. The mean ratio of the gaze and the postural movement are

shoun in Figure 2 and Figure 3 , respectively. The ratio of the gaze movements seemed to differ between the attractive and

non-attractive fixation mark conditions. When only the fixation mark moved, infants moved their gaze more in the attractive fixation mark condition than in

the non-attractive fixation mark condition. This result indicates that the attrac 
tive fixation mark was actually attractive to the infants in this study. The ratio of the postural movements showed that infants moved their body

with the stimulus to which infants did not pay attention. In general, postural

movements were in response to the movement of background rather than

movement of a fixated stimulus. On the moving background, postural suay

increased with the movement of the attractive fixation mark than the movement

of the non-attractive fixation mark. These results differ from results with adults

showing postural movements in response to the movement of fixated stimulus. In $1.0 \mathrm{C}$ : CU E a.1 CU >0 a.1 :5 CU a. $4 \mathrm{~N} \sim \sim \sim$ attractive fixation CU a.2 $\sim$ non-attractive fixation. J! D.D 00 :C $\sim 0:$ : Figure 2. Mean ratios of the gaze movements. Error bars indicates standard devia

tions. $1 / 11.0^{\circ}$ attrilctive fixatio $n$ (J I: $\cdots$ non-attractive fixatio. 4) E 0.84) >0 0.6:e ${ }^{\infty}$ $0.4:::: s 1 / 100.20 . \ldots .00 .00:$; to $0:$ :

Figure 3 . Mean ratios of the posture movements. Error bars indicates standard dev

1982. D. N. Lee \& E. Aronson. Visual proprioceptive control of standing in hu

man infants. Perception and Psychophysics, 15, 529-532, 1974. D. N. Lee \& J. R. Lishman: Visual proprioceptive control of stance. Journal

of Human Movement Studies, 1, 87-95, 1975. 0. Dullier, B. G. Bardy, T. A. Stoffregen \& R. J. Bootsma. Postural coordi nation in looking and tracking tasks. Human Movement Science, 21, 147-167,

2002. F. H. Previc \& M. Donnelly. The effects of visual depth and eccentricity on

manual bias, induced motion, and vection. Perception, 22, 929-945, 1993. F. H. Pre vic \& T. J. Mullen. A comparison 
of the latencies of visually in

duced postural change and self-motion perception. Journal of Vestibular Re

search, 1,317-23,1991. Acknowledgements. This study was supported by PRESTO, JST and a

Grant-in-Aid for scientific research (18300090, 20539004) from JSPS.

(6) 2009 Taylor \& Francis Group, LLC Body Suay in the Processes of Perceiving various Visual Environmental Structures Chih-Mei (Melvin) Yang \& Chia-Chun Huang Department of Physical Education, National Taiwan Normal University, Taipei, Taiwan The coupling between perception and action allous for successful behavior

in an environment. Affordances are opportunities for behavior that reflect the

relationship between action-relevant properties of the environment and the ac

tion producing capabilities of an individual Gibson, 1979; Stoffregen, 2003; cf.

Turvey, 1992). Furthermore, from the perspective of ecological psychology,

animals act to perceive and perceive to act, so they can promote their perception

of affordances by way of action. Optic flow elicited by the movements of the

individual or environment moving objects can provide the specific information

of the environment (Gibson, 1979), and individuals can use it to perceive affor

dances. It is impossible to stand without some degree of body sway. Traditionally,

body suay was regarded noise while keeping balance.

However, Riccio and

Stoffregen (1991) thought that body sway might provide functions other than 
just maintaining balance. They pointed out that the body sway can facilitate per

ception of the suprapostural information. This study was conducted to investigate the change in body sway in the

processes of perceiving various visual eilvironment structures. The research

question was: Is the different body sway generated when individuals perceive

different environmental structures? Based on the theoretic frame of the perception-and-action coupling, we hy

pothesized that in different environmental conditions, individuals would employ

different body sway for using optic flow to perceive more information. Method There were 12 young adults (age 20-40) who participated in this experi

ment. They were healthy, had normal vision, and reported no vestibule or bal

ance-related disease. A Polhemus LIBERTY system and a MotionMonitor mo

tion capture system were used for collecting body sway data. The experimental

set-up included five different colors of surround curtains (black, white, gross

mosaic, and fme mosaic, see Figure 1). Figure I.

Experimental set-up. The participants were required to stand naturally for 40 seconds in 6 conditions: 1) close eyes; and open eyes facing the following environmental structure: 2) normal room structure; 3) all-white curtain; 4) all-black curtain; 5) fine mosaic curtain; and 6) gross mosaic curtain. Participants completed all conditions standing on the ground. In addition, they completed conditions 2 through 5 while standing on an $8 \mathrm{~cm}$ wide by $40 \mathrm{~cm}$ long bar set in both a medial-lateral and anterior-posterior orientation (see Figure 1). There were 7200 points of body positions recorded at $240 \mathrm{~Hz}$ during the middle 30 seconds in each trial. Standard deviations (SDS) for each trial were calculated for body sway, and then 
were statistically analyzed by one-way repeated design ANOVAS in standing conditions and sway directions separately. Results and Discussion 1. Standing on the ground Body sway did not differ in the five experimental surroundings $(F(4,55)=$

$0.315, P=.580,112=.014)$ ). This result shows that the body suay of participants

was not influenced by the change in surroundings and the hypothesis was not supported. But another possibility for this finding is that this it is quite easy to induce body sway in this condition. See Figure 2. $1.4 \mathrm{e} 1.2$ " $" 1$ ' $x$ ' 1 ' 0.8$)(.500 .6<$ II '0 0.4 c til $\sim 0.2$ a Normal white Black Gross mosaic Fine mosaic Surrounding conditions Figure 2. Body sway in standing on the ground condition.

2. Standing on the anterior-posterior set wooden bar There were differences in body sway while standing on the barest in the an

terior-posterior direction. Such differences shous that the body sway is signifi

cantly more stable while facing the gross mosaic surrounding $(F(4,55)=4.786$,

$\mathrm{P}<.05,112=.232)$. This result means the different surroundings do induce the

counterparts in suay. See Figure $3.3 \mathrm{E} 2.5 \sim 111 \cdot x$ " $2 \times$. !: 1.50 VI (5 $1<:$ : " $1 " 0.5 \% 0$

Normal white Bl ack Gross mosaic Finemosaic surrounding conditions Figure 3 . Body sway in standing on the anterior-posterior set wooden bar. $2.5 \mathrm{E} 2 \sim 1$ " ' $x$ '" $1.5 \times=0$ VI 1 ' 0 C: $111 \sim 0.5$ O Normal White Black Gross mosa ic Fine mosaic surrounding conditions Figure 4. Body sway in standing on the mediolateral set wooden bar. 3 . Standing on the mediolateral set wooden bar This result is similar to the last condition. It shows that there is significantly different body sway while facing different structures of surroundings $(\mathrm{F}(4,55)=$

4.630, $P<.05,, ., 2=.214)$. See Figure 4. Based on the data from the experimental manipulations, we concluded that individuals exert different body sway to perceive the information of the envi 
Gibson, J. J. (1979). The ecological approach to visual

perception. Hillsdale, NJ:

Laurence Er lbaum.

Riccio, G. E., \& Stoffregen, T. A. (1991). An ecological theory of motion sickness

and postural instability. Ecological Psychology, 3 , $195-240$.

Stoffregen, T. A. (2003). Affordance as properties of the animal-environment

system. Ecological Psychology, 15, 115-134.

Turvey, M. T. (1992). Affordances and prospective control: fn outline of the

ontology. Ecological Psychology, 4, 173-187. (c) 2009

Taylor \& Francis Group, LLC Body Sway and Visual

Performance at Sea Yawen Yu 1 , Yasunobu Katsumata $2, \&$

Thomas A. Stoffregen l luniversity of Minnesota,

2Toyohashi University of Technoiogy, Japan Postural

control actions serve to maintain the body's center of mass

above

the base of support, which prevents falling. Body sway

moves the center of

mass but it also moves the head and, thereby, influences

the position and motion of the eyes. For this reason, sway

can either inhibit or support stabilization of

the eyes relative to the illuminated environment. We have

shown that the magni

tude of body sway is reduced during performance of demanding visual tasks, such as reading (Stoffregen et aI., 2000), and that such reductions are related to

ocular stabilization, rather than to cognitive components of tasks (Stoffregen et

aI., 2007). In the present study, we examined postural stabilization of vision in a situation involving motion of the surface of support. We stand and walk by pushing against the support surface. The kinematic

consequences of a given muscle contraction are influenced 
by the dynamics of

the support surface, such as its length, rigidity, and friction (Riccio \& stoffre

gen, 1988). A special type of support surface dynamics occurs when the support

surface is moving, as happens in vehicles (Stoffregen \& Riccio, 1988). A clas

sic instance of this case is life at sea. "Getting your sea legs" is one of the oldest

types of perceptual-motor adaptation known to humanity. Impelled by wind and

waves, ships are in continuous oscillatory motion. Unlike an icy sidewalk, we

cannot escape from the challenging dynamics of a ship; we must cope with the

ship's motion continuously. For this reason, ships make an ideal laboratory for

the study oflong-term perceptual-motor adaptation. We studied experienced maritime creumembers on a ship at sea. Relations

between body motion and the performance of visual tasks are of increasing im

portance in naval applications, as work becomes increasingly focused on com

puter interaction. While at sea, standing participants performed difficult and

easy visual vigilance tasks. We predicted that sway would be reduced during

performance of the difficult task, relative' to stance during performance of the

easy task (cf. Stoffregen et aI., 2007). We also varied stance width, that is, the

distance between the feet during stance. Wider stance is associated with reduc 
tions in the magnitude of spontaneous postural suay (e.g., Day et aI., 1993). We

predicted that the magnitude of suay would be negatively related to stance

width. Method The study was conducted aboard the $R / V$ Thomas G. Thompson, during a 10-day cruise that began in Samoa and ended in Hobart, Tasmania. The

Thompson was $84 \mathrm{~m}$ long and displaced 3000 tons, and cruised at 11 knots. Ten

creumembers participated on a volunteer basis. Crew ranged in age from 26 to

60 years, and had from 5 to 30 years experience working at sea. Postural data were collected using a force plate (AMTI). Visual stimuli

were generated using custom software on a PC laptop, and were presented on a 19 -inch flat screen video monitor. The video monitor was affixed to a cabinet

so that its center was $1.7 \mathrm{~m}$ above the floor. Participants stood on the force plate with their heels on a line that was $1.0 \mathrm{~m}$

from the screen. Lateral foot placement was determined by three pairs of lines

on the plate, such that the heels were $5 \mathrm{~cm}, 17 \mathrm{~cm}$ or 30 cm apart. Participants

performed visual vigilance tasks. Visual targets consisted of pairs of lines that

appeared on the screen, against a white bckground. In each pair, the lines ei

ther were the same length (distracters) or were of different lengths (critical sig

nals). Targets appeared for $200 \mathrm{~ms}$, and there was one target per second. For the

Easy task, the lines were solid black. For the Hard task, the lines were pale 
gray. The hard task was equivalent to the one used by Stoffregen et al. (2007).

The sequencing of distracters and critical signals was randomized. There were

20 critical signals per trial. Each trial was $60 \mathrm{~s}$ long. Participants held a wire

less mouse. They were instructed to ignore distracters, and to push the left but

ton on the mouse for each critical signal. The laptop recorded the button

presses. On each day, we used a $2 \times 3$ design with one trial per participant in

each of six conditions. Each trial was $60 \mathrm{~s}$ long.

Participants were tested each

day for 4 days. Easy and Hard trials were blocked, and stance widths were ran

domized within blocks. During testing, sea state varied from 3 to 4 con the

Beaufort scale; Beer, 1997) on Days 12 , from 2 to 3 on Days 34 . We evaluated visual task performance using signal detection theory. The

rates of hits (correct button presses) and false alarms (incorrect button presses)

were combined to yield d', a widely used measure of vigilance performance

(Craig, 1984). We assessed postural activity in terms of the positional variability

of the COP in the AP and ML axes. Results and Discussion The overall mean for d' was 4.28 for the Easy task, and 3.55 for the Hard

task. These means differed, $F(J, 6)=16.16, p=.007$, confirming that the Easy

task was less difficult than the Hard task. $5^{\sim} 4^{\sim} u$ c " $3 \mathrm{E} \ldots{ }^{*} \ldots{ }^{*} 2 \mathrm{Q}$, Har'd '" : = DEasy

III :> 1 a Easy Task Figure 1. Visual performance 
(d'), showing the effect of visual task. For postural sway, the results are summarized in Figure 2. For movement

in the body's AP axis, analysis of variance revealed a significant main effect of

task, $F(1,9)=5.37, p=.046$, and a significant main effect of day, $F(3,7)=17.91, p$

$=.00$ I. The main effect of stance width was not significant, and there were no

significant effects in the ML axis. The main effect of task confirmed our predic

tion, and replicates our land-based studies relating postural control to visual per

formance. The absence of a main effect of stance width on sway suggests that

wider stance may not be used to minimize sway at sea. 0.05

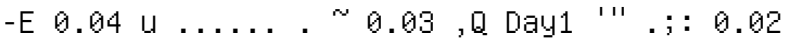
Day2 '" > Q", 0 Day3 ด u 0.01 o Day4 @ Easy Hard Task Figure 2. Positional variability of the CoP in the body's AP axis for the Easy and

Hard tasks, as a function of days. Historically, work at sea has consisted primarily of physical labor. Increa

singly, however, physical labor is being replaced by human-computer interac

tion, much of which consists of the monitoring of computer displays. Thus, our

visual vigilance tasks are relevant to significant components of contemporary

work at sea. Dur results demonstrate that postural control actions can be tuned

in ways that tend to support visual performance and that such tuning exists in

creumembers who are fully acclimated to shipboard conditions. Ships offer a

natural laboratory for the study of perceptual-motor adaptation under dynamic 
conditions. Research of this kind can have direct relevance to issues of crew

Beer, T. (1997). Environmental oceanography. Boca Raton, FL: CRC Press.

Craig, A (1984). Human engineering: The control of vigilance. In J. S. Warm (Ed.), sustained attention in human performance (pp. 247-282). New York: Wiley.

Day, B.L., Steiger, M.J., Thompson, P.D., \& Marsen, C.D. (1993). Effects of vision and stance width on human body motion when standing: implications for afferent control of lateral sway. Journal of Physiology, 469,479-499.

Riccio, G. E., \& Stoffregen, T. A (1988). Affordances as constraints on the control of stance. Human Movement Science, 7, 265-300.

Stoffregen, T. A, Hove, F., Bardy, B. G.; Riley, M., \& Bonnet, C. T. (2007). Postural stabilization of perceptual but not cognitive performance. Journal of Motor Behavior, $39,126-138$.

Stoffregen, T. A, Pagulayan, R. J., Bardy, B. G., \& Hettinger, L. J. (2000). Modulating postural control to facilitate visual performance. Human Movement Science, $19,203-220$.

Stoffregen, T. A, \& Riccio, G. E. (1988). An ecological theory of orientation and the vestibular system. Psychological Review, 95, 3-14. Acknowledgements. We thank Captain Alan McClenaghan and Daniel

Schwartz, University of Washington School of Oceanography, who made this

study possible. 